\title{
Modelling multi-strain pathogen dynamics
}

Inferring between-strain interactions \& assessing vaccine-induced replacement

Irene Man 
The work in this thesis was financially supported by the Strategic Programme of the National Institute of Public Health and the Environment, the Netherlands.

Cover design: Evy Ansems

Printing: Optima Grafische Communicatie

ISBN: 978-94-6361-602-7

(C) 2021, Irene Man

All rights reserved. No parts of this thesis may be reproduced or transmitted in any form or by any means without prior permission from the author. 


\section{Modelling multi-strain pathogen dynamics}

Inferring between-strain interactions and assessing vaccine-induced replacement

\section{Modellering van dynamiek van multi-stam pathogenen}

Afleiding van interacties tussen stammen en beoordeling van stamvervanging door vaccinatie

(met een samenvatting in het Nederlands)

\section{Proefschrift}

ter verkrijging van de graad van doctor aan de Universiteit Utrecht op gezag van de rector magnificus, prof. dr. H. R. B. M. Kummeling, ingevolge het besluit van het college voor promoties in het openbaar te verdedigen op dinsdag 16 november 2021 des middags te 4.15 uur

door

\section{Yi Sum Irene Man}

geboren op 8 december 1990 te Hong Kong 
Promotor:

Prof. dr. M. E. E. Kretzschmar

Copromotor:

Dr. J. A. Bogaards 
Assessment committee:

Prof. dr. J. A. P. Heesterbeek

Prof. dr. M. J. M. Bonten

Prof. dr. J. A. M. Borghans

Prof. dr. H. C. Boshuizen

Prof. dr. J. Berkhof 


\section{Table of contents}

Chapter 1: General introduction 1

Chapter 2: Approximate likelihood-based estimation method of multiple-type pathogen interactions: an application to longitudinal pneumococcal carriage data 25

Chapter 3: Inferring pathogen type interactions using crosssectional prevalence data: opportunities and pitfalls for predicting type replacement 59

Chapter 4: Reconstructing heterogeneous pathogen interactions from co-occurrence data via statistical network inference $\quad 83$

Chapter 5: Capturing multiple-type interactions into practical predictors of type replacement following human papillomavirus vaccination

Chapter 6: Human papillomavirus genotype replacement: still too early to tell?

$\begin{array}{lll}\text { Chapter 7: } & \text { General discussion } & 197\end{array}$

Supplements 225

Nederlandse samenvatting . . . . . . . . . . . . . . . . . . . . . 226

Acknowledgements . . . . . . . . . . . . . . . . . . . . 231

Curriculum vitae . . . . . . . . . . . . . . . . . . . . . . 233

List of publications . . . . . . . . . . . . . . . . . . . . . . . . . . . 234 
Chapter 1

General introduction 


\section{Tangled life}

In life of every form and on every scale, organisms interact: birds do it; bees do it; even bacteria and viruses do it. Not only do organisms interact with organisms of their own species (birds with birds; bees with bees), some organisms also interact with organisms of other species (birds with bees [1]; bees with bacteria [2]; bacteria with birds [3]) and so creating fascinating networks of life.

When organisms interact, there is often something to be gained or lost. In ecology, different categories of interactions have been defined to describe how organisms are affected when they interact (Figure 1.1] [4]. In particular, two of these categories describe interactions that result in symmetrical effects on the interacting parties: mutualism and antagonism, which are also referred to as synergy and competition. With synergistic interaction, the involved parties benefit from each other's presence, for example, by collective production and consumption of a common good or creation of an environment that is beneficial for both. By contrast, competing organisms disadvantage one another, for example, by direct attacks or competition for a common resource. Even more categories of interactions can be defined when also considering asymmetrical combinations of effects on the interacting organisms (Figure 1.1, e.g. parasitism, in which one party benefits at the expense of the other party. Lastly, when organisms (co-)exist without either benefiting or harming one another, we speak of ecological independence.

organism 1

organism 2

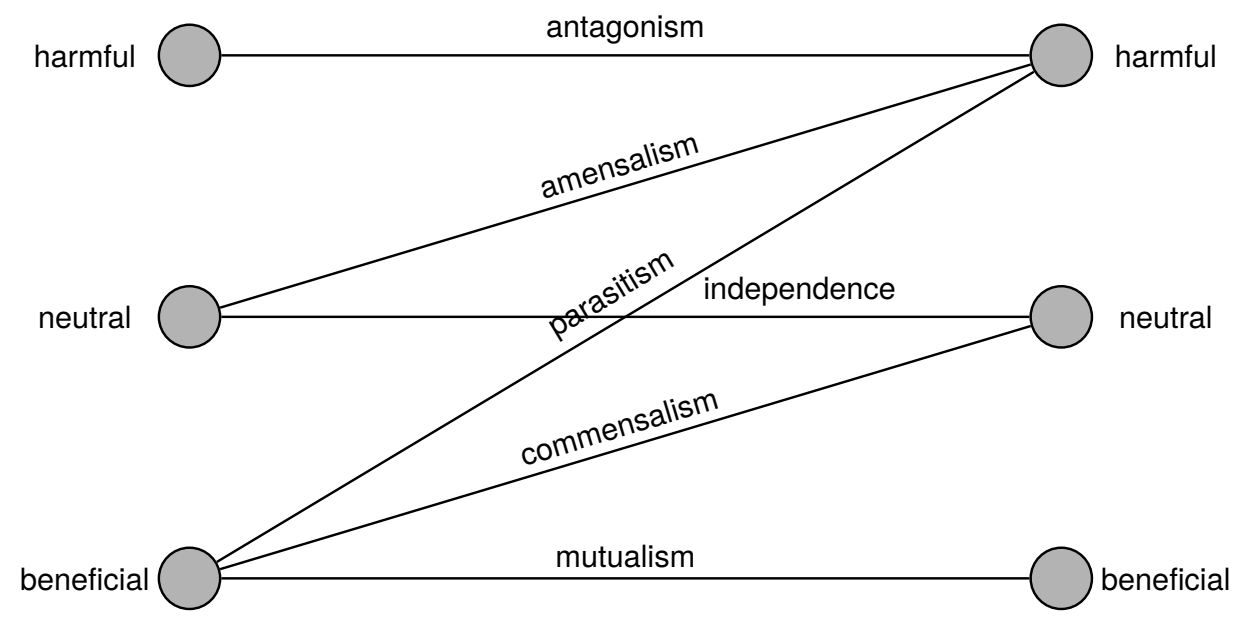

Figure 1.1: Six categories of ecological interactions defined by the combinations of effects experienced by the involved organisms. These categories can also be used to describe interactions between species of organisms, such as pathogen species or pathogen strain species. 
As a result of a myriad of ecological interactions between different organisms, their existences are interconnected. Likewise, the thriving and survival of different groups of organisms as species are entangled. Cooperating species flourish together, while competing species could threaten each other's survival. When the competition is too intense, one species could even drive another one to extinction. Amidst a multitude of interacting species, it is hence not surprising that a variety of interesting spatial and temporal patterns of co-occurrence could emerge.

\section{The central research question}

As life on earth is much entangled, intervening upon one species could lead to radical changes to the occurrence of other species. Put central in this thesis is the intervention vaccination against human pathogens and the question of whether and how vaccination against a pathogen species or a group of pathogen species would affect the occurrence of other, non-targeted pathogen species.

\section{A hypothetical world with two pathogen species}

As a first attempt to address this central research question, let us imagine a world with two pathogen species, both infecting the same host population. In this hypothetical world, if we start vaccinating the host population against one of the two pathogens, we may wonder what would happen to the other, non-targeted pathogen.

Crudely, there are three qualitatively different outcomes, and which one of the three occurs depends on how the two pathogens interact. If the two pathogens co-exist independently (e.g. because they infect distinct parts of the host's body), vaccination should not affect the occurrence of the non-targeted pathogen. If, instead, the two pathogens interact synergistically, vaccination would lead to a decrease in the occurrence of the non-targeted pathogen. This is a result of the loss of synergy from the vaccine-targeted pathogen. This scenario is favourable for the host population, as the disease burden stemming from the non-targeted pathogen would also decrease. However, if the two pathogens interact competitively, vaccination could trigger an increase in the occurrence of the non-targeted pathogen. This occurs as the non-targeted pathogen is released from the competition with the targeted pathogen, i.e. competitive release. As a result, the occurrence of the targeted pathogen could be partially or even completely replaced by that of the non-targeted pathogen. This phenomenon is referred to as vaccine-induced replacement. Such a scenario is unfavourable for the host population, as the disease burden caused by the non-targeted pathogen would also increase. 


\section{Real-life examples of pathogen interactions and vaccination}

Vaccine-induced replacement is not just a theoretical possibility but a realistic outcome that has happened to real-life vaccination. The practice of deliberate immunization against human pathogens was first formally introduced for the smallpox virus [5]. Being the first pathogen to be vaccinated against, smallpox was also the first pathogen for which possible effects on other, non-targeted pathogens were considered. It was feared that vaccination of smallpox would trigger competitive release in other, closely related poxviruses, in particular, cowpox and monkeypox [6]. Before the introduction of vaccination, these other viruses were mainly circulating in animals, causing sporadic cases of diseases in humans. When smallpox vaccination was introduced, the occurrence of these other poxviruses indeed increased in human populations [7-9].

It turned out that different poxviruses were competing through cross-immunity for susceptible human hosts [10]. Before the introduction of smallpox vaccination, when smallpox was still widely circulating, humans were continuously exposed to the smallpox virus and so building up immunity against it. Interestingly, because the smallpox virus and the other poxviruses were much alike, in particular the cowpox and monkeypox viruses, immunity for smallpox also provided a degree of "cross"-immunity against these other poxviruses (and vice versa). When the circulation of smallpox was stopped by vaccination, the build-up of cross-immunity against these other poxviruses also ceased. This loss of cross-immunity then left the human populations more susceptible to the other poxviruses, resulting in vaccine-induced replacement.

Another fascinating fact about vaccine-induced replacement in poxviruses due to smallpox vaccination was revealed when smallpox was eradicated and the vaccination for it ceased. Although vaccine-induced replacement already started to occur while smallpox vaccination was still in place, it only became more pronounced after the cessation of vaccination [10, 11]. The reason for this was that also the vaccine itself provided a degree of cross-protection against the other poxviruses, which was again by virtue of the likeness between poxviruses [12].

This suggests a second factor that may influence the impact of vaccination on the occurrence of pathogens that are not targeted by the vaccine. Besides how the targeted and non-targeted pathogens interact (e.g. competitively or synergistically), the outcome of vaccination for a non-targeted pathogen is also influenced by the degree of cross-protection the vaccine provides for it, which can act as a counterforce against replacement. Note that throughout this thesis we use the term "non-targeted" to indicate the fact that a pathogen is not used for the construction of the vaccine, and hence not intended to be targeted, and not whether the vaccine actually provides protection for it.

Except competition mediated by cross-immunity, which was held responsible for competitive release in the smallpox example, there are other possible mech- 
anisms by which real-life pathogens may compete. For instance, some bacteria that occupy the same part of the human body, e.g. Streptococcus pneumoniae and Staphylococcus aureus, compete for space or other kinds of resources, or attack one another by excreting toxic substances [13, 14]. In fact, pathogens are engaging in competitive interaction when causing a fatal outcome for the infected host; a deceased host is no longer available for infection with another pathogen [15]. These other modes of competitive interaction also have the potential to lead to competitive release when vaccination is introduced against some of the interacting pathogen species.

Vaccine-induced replacement could also emerge in the form of newly mutated variants of the vaccine-targeted pathogen. In reality, each pathogen species is a collection of similar but non-identical organisms that may change over time through evolutionary processes. Amidst competitive interactions within a pathogen species, vaccination could intensify the selective pressure and so induce emergence and replacement of novel pathogen variants against which the vaccine is less efficacious. For instance, such replacement by emerging variants has been observed for Bordetella pertussis and could be a serious threat for the newly introduced vaccination programmes against the novel severe acute respiratory syndrome coronavirus 2 [16, 17]. However, it should be noted that the emergence of vaccine-resistant variants is relatively rare as compared to the emergence of other forms of drug resistance [18, 19].

Fortunately, competition between pathogens is not the only possibility, and vaccine-induced replacement needs not to be a necessary outcome. For the majority of pathogens causing childhood diseases, including measles, polio, diphtheria, mumps, and rubella, vaccination does not seem to have altered much the ecology of other human pathogens, as far as we know [10]. This likely reflects a limited degree of interaction between the targeted and non-targeted pathogens.

As for examples of synergistic interactions between human pathogen species, the pairing of influenza and Streptococcus pneumoniae is one that is often mentioned [20]. It has been suggested that individuals infected with either one of the two pathogens might become more susceptible to the other pathogen, and that transmission of either one might be enhanced during co-infection [21]. While the exact mechanism of cooperation is still debated, vaccination against either one is believed to decrease the risk of disease from the other [22, 23]. Similarly, infection with the human immunodeficiency virus (HIV) is capable of compromising the human immune system, making the infected host more susceptible to other pathogens [24]. Hence, preventing HIV infection by vaccination would also reduce the occurrence of many other diseases [25]. However, despite the long process of vaccine research, no effective vaccine for HIV has been discovered yet.

As a final example, the so-called phenomenon of "original antigenic sin", which is in a way the opposite of cross-immunity, is also a possible mode of syner- 
gistic interactions [26]. For some pathogens, the first infection can prime the host's immune system to such an extent that, when the host encounters a similar pathogen the next time, it may erroneously identify the new pathogen as the "original" pathogen. The immune system could then occupy itself with the wrong response strategy to the advantage of the new pathogen. For instance, this phenomenon has been reported for variants of influenza and dengue [26] 27]. At the same time, cross-immunity, which acts as a form of competition, has also been reported for variants of these viruses [28, 29]. Hence, it is not straightforward how the different forms of interactions between variants would modulate the impact of vaccination [30].

\section{The central research question at the strain level}

The central research question of whether and how vaccination would affect the non-targeted pathogen species is particularly relevant at the strain level. In this thesis, a strain (or a variant, or a type) refers to a subspecies within a pathogen species among which the members are more alike, e.g. in terms of genetics or serologic profile, as compared to the members of other subspecies [31]. In a sense, all pathogens are multi-strain, as there is variation within every pathogen species. However, we use the characterization "multi-strain" here to emphasise the fact that it is a pathogen of which only a selected number of strains are being targeted by a vaccine (where the number of strains targeted is referred to as valency).

For many multi-strain pathogens, effective vaccines have only been successfully developed based on strain-specific characteristics. For instance, the bacterium Streptococcus pneumoniae, which comprises more than 80 distinct serotypes, can be targeted by pneumococcal conjugate vaccines (PCVs), which contain purified capsular polysaccharides that bind to serotype-specific surface antigens 32 33]. To date, three PCVs have been used, targeting 7,10 , or 13 serotypes. The human papillomavirus, another example of a multi-strain pathogen, consists of more than 200 genotypes, defined by the differences in the L1 capsid gene of their DNA sequence [34]. Three prophylactic vaccines are currently available, containing particles that resemble the L1 capsid proteins of 2, 4, or 9 genotypes [34-36]. Haemophilus influenzae, Neisseria meningitidis, influenza and rotavirus are a few more examples of multi-strain pathogens with vaccines that target only subsets of strains (Table 1.1. In the future, even more vaccines targeting only a subset of strains of a pathogen may become available.

Given the high level of similarity between strains of the same pathogen, it is likely that different strains of the same pathogen do not exist in complete independence. Therefore, it is also not unlikely that vaccination against a subset of strains may affect the occurrence of the non-targeted strains. With this consideration, we 
Table 1.1: A selective list of vaccines targeting a subset of strains of a multi-strain pathogen.

\begin{tabular}{|c|c|c|c|}
\hline Pathogen species & Number of main circulating strains & Vaccine & Valency \\
\hline Haemophilus influenzae & 5 serotypes and non-typeable & $\mathrm{Hib}$ & 1 serotype \\
\hline Neisseria meningitidis & 13 serogroups & $\begin{array}{l}\text { Bexsero } \\
\text { Nimenrix }\end{array}$ & $\begin{array}{l}1 \text { serogroup } \\
4 \text { serogroups }\end{array}$ \\
\hline Streptococcus pneumoniae & $\sim 80$ serotypes and non-typeable & $\begin{array}{l}\text { Prevnar7 } \\
\text { Synflorix } \\
\text { Prevnar13 }\end{array}$ & $\begin{array}{l}7 \text { serotypes } \\
10 \text { serotypes } \\
13 \text { serotypes }\end{array}$ \\
\hline Human papillomavirus & $\sim 40$ sexually transmitted genotypes & $\begin{array}{l}\text { Cervarix } \\
\text { Gardasil } \\
\text { Gardasil9 }\end{array}$ & $\begin{array}{l}2 \text { genotypes } \\
4 \text { genotypes } \\
9 \text { genotypes }\end{array}$ \\
\hline Rotavirus & 6 main genotypes & $\begin{array}{l}\text { Rotarix } \\
\text { RotaTeq }\end{array}$ & $\begin{array}{l}1 \text { genotype } \\
5 \text { genotypes }\end{array}$ \\
\hline Influenza & $\begin{array}{l}4 \text { main strains, representing influenza } \\
\text { A subtypes and influenza B lineages }\end{array}$ & $\begin{array}{l}\text { Updated } \\
\text { twice a year }\end{array}$ & $\begin{array}{l}3 \text { or } 4 \\
\text { subtypes }\end{array}$ \\
\hline
\end{tabular}

set the objective of this thesis to be the investigation of the central research question at the strain level. In other words, the central research question is whether and how vaccination against a subset of strains of a pathogen would affect the occurrence of the non-targeted strains of the same pathogen.

Furthermore, we will limit the scope of our investigation in two respects. Firstly, we will disregard interactions between the vaccine-targeted pathogen and other pathogens, and focus on the impact of vaccination on the non-targeted strains of the targeted pathogen only. Although vaccination against some strains of one pathogen may also affect other pathogens, it is reasonable to first concentrate on the interactions between strains within the same pathogen species. Such simplification could be justifiable because the interactions with other pathogen species are negligible relative to the between-strain interaction. Sometimes, such simplification is mainly pragmatic. Secondly, we will mainly consider pathogens for which relevant evolution is negligible on the time scale of vaccination. For fast-evolving multi-strain pathogens, e.g. influenza [37], it is necessary to also consider how the genetic contents of the existing strains change over time, increasing the complexity of the investigation.

\section{Supporting public health decisions}

Being able to assess how vaccination against a multi-strain pathogen affects the occurrence of the different strains of the pathogen is important for many public health decisions. Some of these decisions concern the introduction and implementation of vaccination against multi-strain pathogens. Firstly, there is the decision of whether or not to widely introduce vaccination in a population. If vaccination is introduced and strain replacement does occur, should a programme be retracted or modified? If multiple vaccines targeting different sets of strains are available, which one should be used?

The impact of vaccination against a multi-strain pathogen may also have implications for decisions regarding other public health interventions. As a primary 
prevention intervention, vaccination may in turn affect the impact of other downstream interventions, such as screening and treatment. For HPV vaccination, for example, the need for screening for HPV-related cancers depends much on the occurrence of the remaining non-targeted HPV genotypes after removing the vaccine-targeted strains [38]. Whether and to what extent strain replacement occurs is important for determining the optimal interval between screening rounds. As another example, for pathogens that require treatment with antibiotics, decisions on vaccination may also affect policies around the use of antibiotics. By preventing infection with such pathogens, we also prevent the need for treatment using antibiotics. Hence, in principle, vaccination could lower the risk of emergence of antimicrobial resistance [39]. Therefore, decisions on vaccination may also influence decisions on other public health interventions.

An important criterion to evaluate a given intervention (or to compare different interventions) is by considering the associated impact on the total disease burden. To illustrate, let us revisit the smallpox example. Smallpox vaccination had led to the complete eradication of the virus worldwide. At the same time, it has only caused minor increases in the occurrence of other poxviruses [10]. The total occurrence of diseases caused by all poxviruses in humans has hence reduced considerably. Moreover, the severity of infection with other poxviruses is in general less than that of smallpox [40]. As a result, the impact in terms of the total disease burden of all poxviruses has been substantial. In the context of vaccination against multi-strain pathogens, similar criteria can be defined by the total disease burden of all strains of a pathogen. To evaluate this, it is essential to be able to assess the impact of vaccination on the occurrence of each strain, as well as the strain-specific disease-causing potentials.

\section{Methodological problems and challenges}

Given the public health relevance, it is important to be able to assess the impact of vaccination against multi-strain pathogens on the occurrence of non-targeted strains. In this thesis, we aim to improve the methodology for such assessment in terms of the following three problems:

1. inference of between-strain interaction using pre-vaccination co-occurrence data;

2. prediction of strain replacement using inferred information on between-strain interaction;

3. evaluation of strain replacement using post-vaccination data.

In this section, we introduce each of these three problems and describe, for each one of them, the existing methodologies and the challenges that we aim to ad- 
dress.

\section{Inference of between-strain interaction}

An essential step towards assessing the impact of vaccination on a multi-strain pathogen is to understand whether and how the pathogen strains interact. As between-strain interaction is a key element shaping the co-occurrence patterns of the pathogen strains, data on the co-occurrence patterns allow us to infer how the strains interact. At the miminum, one may wish to know whether the strains interact or not. If there is enough evidence that the strains interact, more details may be of interest, including the nature, mode, and strength of the interaction.

While many types of data may contain information about between-strain interaction, this thesis will focus on presence/absence co-occurrence data of pathogen strains in human hosts. In addition, we restrict the scope to data collected before the introduction of vaccination against the pathogen of interest. Depending on the pathogen, data on different types of occurrences could be relevant. The two types of occurrences that we will mainly consider are the occurrence of colonisation and infection. The former describes an asymptomatic state of carriage, while the latter implies an invasive state in which damage could be inflicted to the host. For bacteria, co-occurrence of colonisation is often more relevant to consider for between-strain interaction (e.g. Streptococcus pneumoniae). For viruses (e.g. the human papillomavirus), it is more suitable to consider interaction on the level of infection occurrence. Furthermore, we will focus on data collected according to the following two forms of study design. The first one is the longitudinal design, for which measurements are made on the same group of individuals at multiple time points. The second one is the cross-sectional design, for which measurements of a group of individuals are made at a single time point.

There are other forms of data containing information about between-strain interaction we do not consider in depth in this thesis. One example is time series data, which consist of measurements of different sets of individuals (instead of the same set of individuals) at multiple time points [21]. Another form is data of non-randomly sampled individuals in a population, such as data obtained from household-based or snowball-sampling studies [41, 42]. Finally, quantitative data indicating the extent of colonisation or infection, e.g. data of bacterial or viral loads $[43,44]$, and data from animal and in vitro experiments are also beyond the scope of this thesis.

Methodologies to infer between-strain interaction from the form of longitudinal and cross-sectional data mentioned above are usually based on estimation of association measures that quantify how the occurrences of different strains are affected by one another [45]. Whether between-strain interaction is present can be tested by investigating whether the estimated association is significant. If the association is significant, the strength and nature of interaction can be derived 
from the numerical value and the sign of association, with synergy indicated by positive association and competition by negative association.

In the cross-sectional setting, such association measures take the form of odds ratios (ORs) comparing the odds of being found with a given strain (or a set of strains) in the presence versus absence of another strain (or another set of strains) [46 47]. Similarly, in the longitudinal setting, between-strain interaction can be estimated in the form of hazards ratios (HRs) that compare the hazards of certain events related to a given strain (or a set of strains) in the presence versus absence of another strain (or another set of strains) [48, 49]. Which event should be used in the definition of an HR depends on the mode of interaction we hypothesise. For example, if we would like to infer information about interaction in the modes of acquisition and clearance, the corresponding HRs should be defined based on hazards of acquisition and clearance events, i.e. the initiation and termination of a period in which a pathogen strain is found in the host.

Estimation of such association measures of between-strain interaction (in the form of either ORs or HRs) can be done through different approaches. Two examples that we will study in more depth in this thesis are multi-state models and network inference models [50-52]. These two approaches are for the longitudinal and cross-sectional settings, respectively. The former has frequently been used for the purpose of inferring between-strain interaction (e.g. interactions between serotypes of Streptococcus pneumoniae [48, 49] and genotypes of the human papillomavirus [53]), while the latter not. In multi-state models, all possible combinations of co-occurring strains define the state space, and all possible events of acquisition or clearance define the transitions between the model states. Interactions between different sets of strains can be estimated by comparing different sets of transition hazards in one single multi-state model. In network inference models, associations between different pairs of strains are also estimated within a single model in terms of conditional dependency, where the conditioning is done on the occurrence of the strains that are not being considered in a pair.

There are diverse challenges related to inference of between-strain interaction. First, a defined association measure may not necessarily match the underlying mode of interaction, possibly leading to biased or false conclusions. For instance, cross-immunity, a mode of between-strain competition, may induce positive association and lead to a false conclusion of synergy when assessed based on the cross-sectional OR of co-occurrence [54-56]. Although it has been shown by simulation that such reverse bias exists, it is not entirely clear how it is generated mechanistically. Besides this example, there may be other modes of interactions that may not match with a given association measure. So far, only little investigation has been done exploring the validity of different association measures under different possible modes of between-strain interaction.

Another challenge related to inference of between-strain interaction is to man- 
age the tension between a detailed description of interaction and computational tractability. To obtain accurate and detailed information about between-strain interaction, complex inference models are needed. Inference of interaction between many pathogen strains can easily become high-dimensional and computationally expensive. For instance, the vast number of combinations of co-occurring strains we encounter in longitudinal data poses a high computational burden on the estimation procedure in multi-state models. Furthermore, the more strains there are, the more interaction parameters corresponding to different combinations of strains could be defined. Between-strain interaction may not only depend on the combination of strains that are currently occurring in the host but also the combination of strains that have occurred in the host and the sequence of thereof 57 . 58]. Hence, inference of interactions may require complex combinatorics and high computational resources.

Lastly, there is the challenge of dealing with confounding due to host heterogeneity. Individuals are inherently different, and the risk of being infected (or colonised) often differs across individuals depending on the individual risk factors. Nevertheless, the risks of acquiring different pathogen strains are likely determined by similar sets of risk factors due to the similar route of transmission and natural history. Hence, an individual at a higher risk for one strain is also expected to be at a higher risk for the other strains. When common risk factors are not adjusted for, estimates of between-strain interaction can be biased. Theoretically, such confounding can be overcome by randomly selecting individuals to expose them to different sets of pathogen strains, but such deliberate exposure of harmful pathogens would not be ethical. Randomised experiments in animals are less controversial but may not fully capture the between-strain interactions manifesting in humans. Inference of between-strain interaction hence necessitates observational studies in humans, in which the presence of confounding is inevitable. Confounding due to common risk factors is an issue that has been widely investigated, with as results a range of statistical approaches for adjustment [59, 60]. Still, there is ambiguity about how confounding due to host heterogeneity itself manifests in the inference of between-strain interaction. For instance, it is unclear how well adjustment of confounding performs amidst indirect strain interactions and for methods that have not often been used for inference of between-strain interaction, e.g. network inference models.

\section{Prediction of strain replacement}

After having inferred information on between-strain interaction, this information can be used to predict how vaccination would affect the occurrence of non-targeted strains. Prediction may concern the qualitative outcome (i.e. no change, increase, or decrease in occurrence) or the quantitative outcome (i.e. the extent of change in occurrence) of the occurrence of non-targeted strains. Furthermore, prediction 
may concern the outcome for individual non-targeted strains or groups of nontargeted strains. As the different pathogen strains are not identical, the outcome needs not to be the same for each (group of) non-targeted strain.

If the pathogen would have two strains only, prediction of strain replacement would be relatively straightforward. As demonstrated by the hypothetical example with two pathogen species (see page 3), the qualitative outcome is determined by the nature of the interaction (i.e. competitive, synergistic, or independent), provided that there is no vaccine-induced cross-protection); the quantitative outcome could be approximated from the extent to which the estimated association measure diverges from the value corresponding to independence.

An important challenge in predicting the outcome of vaccination for a multistrain pathogen is how to combine all information about how the different strains interact. When there more than two strains, information on interactions between different combinations of strains may need to be summarised into a prediction of what would happen to a given strain or a group of strains. For instance, to predict the outcome for a non-targeted strain, one may need to summarise the information on how this given strain interacts with each of the vaccine-targeted strains. In addition, prediction may also require information on how the non-targeted strains interact with each other. For instance, consider a hypothetical case with three strains, of which one is targeted by the vaccine (strain $A$ ) and two are not (strains $B$ and $C$ ). It is possible that non-targeted strain $B$ is affected by vaccination because it interacts with the other non-targeted strain $C$, as the latter also interacts with strain $A$. In this case, the information about the interaction between the two nontargeted strains is also needed to predict the outcome for non-targeted strain B (or C). In addition, when strains interact through multiple modes simultaneously (e.g. through acquisition and clearance), information about the different modes also need to be summarised.

\section{Evaluation of strain replacement}

The third methodological problem we consider in this thesis is how to evaluate the impact of vaccination on the occurrence of non-targeted strains using postvaccination data. The two main forms of post-vaccination data used for the evaluation of strain replacement are considered in this thesis. The first is data on trends in the occurrence of non-targeted strains over time. In particular, we considered data that encompass a period before the introduction of vaccination to establish the pre-vaccination level of occurrence in the non-targeted strains. When such trend data are available, risk ratios, e.g. prevalence ratios (PRs), comparing the occurrence of non-targeted strains between the pre- and post-vaccination eras can be computed [61]. When trend data are not available, an alternative is to evaluate the occurrence of strain replacement based on vaccine effectiveness (VE) against the non-targeted strains. This effect measure compares the occurrence of non- 
targeted strains between vaccinated and unvaccinated individuals, both measured in the post-vaccination era [62, 63]. The rationale behind such a comparison is to detect possible signs of competitive release in vaccinated individuals, as these individuals may be subject to an increased risk for non-targeted strains due to the reduced susceptibility to vaccine-targeted strains.

While these effect measures have often been used, it is not well investigated how robust they are in evaluating strain replacement. Multi-strain occurrence is complex and dynamic. Whether and to what extent strain replacement occurs may vary over time and across different subpopulations (e.g. age groups, risk groups, or vaccinated/unvaccinated individuals). It is not straightforward how well an estimated value of an effect measure derived at a given time and in a given subpopulation corresponds with the overall outcome of strain replacement at the population level.

\section{Two multi-strain pathogens as case studies}

To make the analyses of the posed methodological problems and challenges more concrete, two multi-strain pathogens are taken as case studies in this thesis: Streptococcus pneumoniae and the human papillomavirus. For both of these multi-strain pathogens, polyvalent vaccination was widely introduced in the first decade of the 21 st century. At the time of introduction, investigators in both fields were cautious in claiming that strain replacement would occur. However, the experiences for the two pathogens diverged markedly within a few years after the onset of vaccination. While serotype replacement in pneumococcus has been clearly observed within a few years [64], emergence of HPV genotype replacement is still under debate [65]. Due to these diverging developments, the corresponding research fields have been facing different research questions. In this section, we briefly introduce these two multi-strain pathogens and summarise the current state of knowledge regarding between-strain interaction and the occurrence of strain replacement for each of them.

\section{Streptococcus pneumoniae}

Streptococcus pneumoniae (pneumococcus) is a bacterium that colonises the upper airways and the oral cavity of the human body [66]. It is transmitted through close-proximity contact. Colonisation, also referred to as carriage, is usually asymptomatic, but when the bacterium spreads to otherwise sterile sites, invasive pneumococcal infections can give rise to various diseases including otitis media, pneumonia, bacteremia, and meningitis, with particularly high morbidity and mortality in infants and the elderly [67, 68].

In 2000, the first pneumococcal conjugate vaccine (PCV) was licensed, target- 
ing seven serotypes $(4,6 \mathrm{~B}, 9 \mathrm{~V}, 14,18 \mathrm{C}, 19 \mathrm{~F}$, and $23 \mathrm{~F})$ that have high potential to cause invasive pneumococcal diseases (IPD). Around 2008, two higher-valent vaccines became available, targeting three $(1,5$, and $7 F)$ and six $(1,3,5,6 \mathrm{~A}, 7 \mathrm{~F}$, and 19A) additional serotypes that are also important for IPD. Currently, the heptavalent vaccine is no longer available, and either of the two remaining vaccines is used in most Western countries.

Already in the clinical trial phase, there were signs hinting at the occurrence of serotype replacement [69, 70]. However, it was then still unclear to what extent replacement would occur in terms of carriage and disease once vaccination would become widely implemented in populations. Within a few years after the introduction of mass vaccination in various countries, it became clear that carriage of vaccine-type pneumococcus was more or less completely replaced by the carriage of non-vaccine-type pneumococcus [71]. As a result, the overall pneumococcal carriage has remained roughly the same. Fortunately, although the occurrence of IPD due to non-vaccine types did increase, the total burden of IPD did decrease due to the lower invasiveness of the non-vaccine types [72].

Meanwhile, through epidemiological and experimental studies, the existence of between-serotype competition was investigated in more depth, and various possible modes of competition were identified. For instance, based on longitudinal data of pneumococcal carriage in humans, it was found that established colonisation with one serotype of pneumococcus could impede the acquisition and accelerate the clearance of another serotype [48, 49, 73, 74]. In addition, some studies in humans and mouse models suggest that previous colonisation with pneumococcus, irrespective of the serotype, could confer immunity against subsequent colonisation of other serotypes, hence inducing competition between serotypes [75, 76].

Still, there are knowledge gaps regarding how pneumococcal serotypes compete. For instance, previous findings of between-serotype competition might have been biased due to the suboptimal sensitivity of the then-used culture-based detection methods [49, 74]. Contemporary molecular-based methods are more sensitive in detecting all serotypes present in a sample, giving rise to more accurate and detailed records of co-occurring pneumococcal serotypes [77,-79]. Data derived from such molecular-based methods could provide more accurate estimates of the modes and strengths by which serotypes interact. However, existing computational approaches to infer between-serotype competition require adjustment to cope with the more accurate but also more complex data obtained using molecular-based methods.

Furthermore, it is not fully understood why the observed patterns of replacement vary across countries and risk groups, in terms of replacing serotypes as well as the extent of replacement in carriage and disease [72, 80, 81]. For instance, while there has been almost no replacement in IPD in the elderly in the 
USA since the switch to PCV13, replacement has been considerable in the UK and many other European countries. Country-specific differences concerning baseline prevalence compositions, surveillance systems, and prevalence of co-morbidities are only able to explain these differences partially [72]. How competitive ability varies across pneumococcal strains is also likely to play a key role herein. A better understanding of between-strain competition is still needed to fully understand and improve the currently adopted vaccines and vaccination programmes [82, 84$]$.

\section{Human papillomavirus}

The second multi-strain pathogen we consider is the human papillomavirus (HPV). HPV is a DNA virus that infects the surface of the human body and spreads through skin-to-skin contact [85]. To date, more than 200 genotypes have been identified, varying in their affinity for infecting distinct types of skin tissues. Around 40 genotypes have been found in the genital and oropharyngeal sites that can be transmitted through sexual contact. These sexually transmitted types are further classified according to the diseases they cause. Low-risk types predominantly cause anogenital or laryngeal warts, while high-risk types have carcinogenic potential [86].

The first HPV vaccine, a quadrivalent vaccine, was licensed in 2006 [87]. It was quickly followed by a bivalent vaccine in 2007 [88], and then a nonavalent vaccine in 2015 [89]. All three vaccines target the two most oncogenic high-risk types, HPV 16 and 18. Both the nonavalent and the quadrivalent vaccines cover two additional low-risk types, HPV 6 and 11, which are held responsible for the majority of anogenital warts [90]. The nonavalent vaccine targets five additional high-risk types that are associated with cervical cancer, HPV 31, 33, 45, 52, and 58. To date, while most countries are still using the bivalent or quadrivalent vaccines, high-income countries are increasingly switching to the newer but more expensive nonavalent vaccine.

As HPV vaccination was introduced several years after the introduction of pneumococcal conjugate vaccines, researchers of the HPV field were alert to the risk of strain replacement [91, 92]. To assess the risk of replacement in HPV, many studies have been conducted to investigate the presence of possible interactions between HPV types. The most compelling evidence for competition between HPV types were findings of mutual suppression of viral loads between genotypes in coinfected hosts and blockage of additional genotypes from already infected cells [43. 44]. However, in studies of HPV at the host level, co-infection of HPV types is common, both in men and women [45, 93, 94]. Infected individuals are often found to be at an increased risk of subsequent infection with other types 95 96]. In general, findings of (comparatively) negative associations between pairs or groups of HPV types are rare and inconsistent, which suggest a limited degree of between-type competition [46, 97-99]. 
Yet, the observed patterns of clustering between HPV types may not exclude the possibility of HPV genotype replacement. First, the clustering pattern of HPV types may stem from host heterogeneity [56, 59]. In general, sexual behaviour is highly heterogeneous. In most studies, statistical techniques have been used to adjust for possible host heterogeneity (e.g. by modelling random or fixed effects that represent individuals' risk for occurrence of any strains) [93]. However, it remains difficult to judge whether findings of positive associations between HPV types are genuine signs of the lack of competition instead of results of residual host heterogeneity. In addition, modelling studies have shown that positive associations between HPV types could also be due to natural cross-immunity between different genotypes, which entails a risk for genotype replacement [54-56].

As pre-vaccination data on the presence of between-genotype interactions are inconclusive, emergence of HPV genotype replacement needs to be evaluated from post-vaccination data. To date, around ten years of data on the population impact of the bivalent and the quadrivalent vaccines have been accumulated and studied. The data show that the prevalence of non-vaccine HPV types 31,33 , and 45 has decreased over time [65, 100]. This evidence against the occurrence of HPV genotype replacement is further supported by findings of positive VE in these types, meaning a lower risk of infection in vaccinated as compared to unvaccinated individuals [62 63]. However, when disregarding HPV 31,33 , and 45 , there is a modest increase in the prevalence of the remaining non-vaccine highrisk types [65, 100]. For some of these non-vaccine types, there have also been findings of negative VE, i.e. a higher risk of infection in vaccinated as compared to unvaccinated individuals [62, 63].

Based on post-vaccination data, it is also debatable whether HPV genotype replacement can already be ruled out. In the case of pneumococcal vaccination, the occurrence of serotype replacement could be observed within several years. Given the slower transmission dynamics and the natural history of HPV infection, more time may be needed for HPV genotype replacement to become noticeable. Furthermore, the interpretation of positive VE as evidence against type replacement is not well investigated. Taken together, there is a need to more carefully assess the interpretation of currently available data and so to predict how the occurrence of non-targeted HPV types will evolve in the future.

\section{Overview chapters}

In this thesis, we aim to improve the methodology of assessing the impact of vaccination against a subset of strains of a pathogen on the non-targeted strains. We do this by evaluating and building on existing methodologies for the three aforementioned problems. The inference problem is covered by Chapters 2, 3 and 4; the prediction problem by Chapters 3 and 5 ; and the evaluation problem by 
Chapter 6.

More specifically, in Chapter 2, we develop a novel computational method to accommodate inference of between-strain interactions from longitudinal data recording the co-occurrence of multiple pathogen strains. Unlike previous methods, it allows analysis of data containing simultaneous co-occurrence of more than two strains [48, 49, 73, 74]. The novel method is validated through a simulation study, in which we investigate various aspects of study design that may bias estimates of between-strain interactions. Subsequently, the proposed method is applied to a pneumococcal carriage dataset, obtained using a molecular-based detection method, to derive new insights into how pneumococcal strains interact.

In Chapter 3, we evaluate the validity of using the OR of co-occurrence, computed from cross-sectional co-occurrence data, as an estimator of between-strain interactions and a predictor for strain replacement. In a transmission model with two strains, we study how well the OR corresponds with the interaction parameters under different nature and modes of between-strain interactions. In addition, we study the mechanisms by which unobserved common risk factors lead to biased estimates of between-strain interactions [54, 56].

The subsequent two chapters extend the results of Chapter 3 from a setting of two strains to a setting with an arbitrary number of strains. Chapter 4 addresses the inference problem and Chapter 5 the prediction problem.

In Chapter 4, we consider the use of network inference models to infer interactions between multiple pathogen strains from cross-sectional co-occurrence data [51, 52]. In a simulation study, we evaluate the performance of a suite of network models in reconstructing heterogeneous interaction networks. We compare the performance of various network inference models and explore their ability to adjust for host heterogeneity.

In Chapter 5, we develop a novel framework to predict strain replacement in a setting with arbitrary numbers of targeted and non-targeted strains. We construct predictors based on ORs and HRs, which can be estimated from cross-sectional or longitudinal co-occurrence data. The framework accommodates the prediction of strain replacement at the strain-specific level as well as for groups of non-targeted strains. Using simulated data, the performance of the proposed predictors is evaluated under different assumptions of how heterogeneous interactions between multiple pairs of strains combine when simultaneously infecting hosts.

In Chapter 6, we evaluate the validity of the prevalence ratio and vaccine effectiveness for non-targeted strains, computed from post-vaccination data, for the evaluation of strain replacement. Using an age-structured transmission model with a vaccine-targeted and a non-targeted strain, we simulate the impact of vaccination under different degrees of competition through cross-immunity and vaccineinduced cross-protection. Based on the simulations, we investigate how well the occurrence of strain replacement corresponds with what is indicated by the two ef- 
fect measures under consideration. To explore the validity of the measures under different settings, we simulate vaccination under different vaccination coverage and evaluation of the two effect measures in different age groups and time horizons.

Finally, in a general discussion in Chapter 7 , we summarise the methodological findings, highlight some overarching principles and future research directions, and discuss the implications for the two case studies and other multi-strain pathogens.

\section{References}

[1] N. M. Waser. "Competition for hummingbird pollination and sequential flowering in two Colorado wildflowers". Ecology 59.5 (1978), 934-944.

[2] Z. Rokop, M. Horton, and I. Newton. "Interactions between cooccurring lactic acid bacteria in honey bee hives". Appl Environ Microbiol 81.20 (2015), 7261-7270.

[3] C. M. H. Benskin, K. Wilson, K. Jones, et al. "Bacterial pathogens in wild birds: a review of the frequency and effects of infection”. Biol Rev Camb Philos Soc 84.3 (2009), 349-373.

[4] M. Begon, J. L. Harper, C. R. Townsend, et al. Ecology. Individuals, populations and communities. Blackwell scientific publications, 1986.

[5] S. Riedel. "Edward Jenner and the history of smallpox and vaccination". Proc (Bayl Univ Med Cent). Vol. 18. 1. Taylor \& Francis. 2005, 21-25.

[6] F. Fenner, D. A. Henderson, I. Arita, et al. Smallpox and its eradication. Vol. 6. World Health Organ Geneva, 1988.

[7] J. G. Breman, M. Kalisa-Ruti, E. Zanotto, et al. "Human monkeypox, 1970-79". Bull World Health Organ 58.2 (1980), 165.

[8] R. M. Vorou, V. G. Papavassiliou, and I. N. Pierroutsakos. "Cowpox virus infection: an emerging health threat". Curr Opin Infect Dis 21.2 (2008), 153-156.

[9] J. O. Lloyd-Smith, D. George, K. M. Pepin, et al. "Epidemic dynamics at the human-animal interface". Science 326.5958 (2009), 1362-1367.

[10] J. O. Lloyd-Smith. "Vacated niches, competitive release and the community ecology of pathogen eradication". Philos Trans R Soc B Biol Sci 368.1623 (2013), 20120150.

[11] A. W. Rimoin, P. M. Mulembakani, S. C. Johnston, et al. "Major increase in human monkeypox incidence 30 years after smallpox vaccination campaigns cease in the Democratic Republic of Congo". Proc Natl Acad Sci U S A 107.37 (2010), 16262-16267.

[12] R. B. Kennedy, I. G. Ovsyannikova, R. M. Jacobson, et al. "The immunology of smallpox vaccines". Curr Opin Immunol 21.3 (2009), 314-320.

[13] D. Bogaert, A. van Belkum, M. Sluijter, et al. "Colonisation by Streptococcus pneumoniae and Staphylococcus aureus in healthy children”. Lancet 363.9424 (2004), 1871-1872.

[14] D. P. Lloyd and R. J. Allen. "Competition for space during bacterial colonization of a surface". J R Soc Interface 12.110 (2015), 20150608.

[15] P. Rohani, D. J. Earn, B. Finkenstädt, et al. "Population dynamic interference among childhood diseases". Proc Biol Sci 265.1410 (1998), 2033-2041.

[16] F. R. Mooi, N. A. van der Maas, and H. E. de Melker. "Pertussis resurgence: waning immunity and pathogen adaptation - two sides of the same coin". Epidemiol Infect 142.4 (2014), 685694. 
[17] D. A. Kennedy and A. F. Read. "Monitor for COVID-19 vaccine resistance evolution during clinical trials". PLoS Biol 18.11 (2020), e3001000.

[18] A. F. Read and M. J. Mackinnon. "Pathogen evolution in a vaccinated world". Evolution in Health and Disease 2 (2008), 139-52.

[19] D. A. Kennedy and A. F. Read. "Why does drug resistance readily evolve but vaccine resistance does not?" Proc Biol Sci 284.1851 (2017), 20162562.

[20] J. A. McCullers. "Insights into the interaction between influenza virus and pneumococcus". Clin Microbiol Rev 19.3 (2006), 571-582.

[21] S. Shrestha, B. Foxman, D. M. Weinberger, et al. "Identifying the interaction between influenza and pneumococcal pneumonia using incidence data". Sci Transl Med 5.191 (2013), 191 ra84.

[22] K. P. Klugman, S. A. Madhi, A. S. Ginsburg, et al. "The role of bacterial vaccines in the prevention of influenza mortality". Lancet Glob Health 6.12 (2018), e1268-e1269.

[23] A. M. Smith and V. C. Huber. "The unexpected impact of vaccines on secondary bacterial infections following influenza". Viral Immunol 31.2 (2018), 159-173.

[24] M. J. Alter. "Epidemiology of viral hepatitis and HIV co-infection". J Hepatol 44 (2006), S6-S9.

[25] G. J. Weverling, A. Mocroft, B. Ledergerber, et al. "Discontinuation of Pneumocystis carinii pneumonia prophylaxis after start of highly active antiretroviral therapy in HIV-1 infection". Lancet 353.9161 (1999), 1293-1298.

[26] T. Francis. "On the doctrine of original antigenic sin". Proc Am Philos Soc 104.6 (1960), $572-$ 578.

[27] S. B. Halstead, S. Rojanasuphot, and N. Sangkawibha. "Original antigenic sin in dengue". Am $J$ Trop Med Hyg 32.1 (1983), 154-156.

[28] N. G. Reich, S. Shrestha, A. A. King, et al. "Interactions between serotypes of dengue highlight epidemiological impact of cross-immunity". J R Soc Interface 10.86 (2013), 20130414.

[29] S. Tamura, T. Tanimoto, and T. Kurata. "Mechanisms of broad cross-protection provided by influenza virus infection and their application to vaccines". Jpn J Infect Dis 58.4 (2005), 195207.

[30] C. J. Worby, J. Wallinga, M. Lipsitch, et al. "Population effect of influenza vaccination under co-circulation of non-vaccine variants and the case for a bivalent A/H3N2 vaccine component". Epidemics 19 (2017), 74-82.

[31] O. Balmer and M. Tanner. "Prevalence and implications of multiple-strain infections". Lancet Infect Dis 11.11 (2011), 868-878.

[32] T. Francis and W. S. Tillett. "Cutaneous reactions in pneumonia. The development of antibodies following the intradermal injection of type-specific polysaccharide". J Exp Med 52.4 (1930), 573-585.

[33] E. Lund and J. Henrichsen. "Laboratory diagnosis, serology and epidemiology of Streptococcus pneumoniae". Vol. 12. Elsevier, 1978, 241-262.

[34] H.-U. Bernard, R. D. Burk, Z. Chen, et al. "Classification of papillomaviruses (PVs) based on 189 PV types and proposal of taxonomic amendments". Virology 401.1 (2010), 70-79.

[35] D. M. Harper and L. R. DeMars. "HPV vaccines - A review of the first decade". Gynecol Oncol 146.1 (2017), 196-204.

[36] R. B. Roden and P. L. Stern. "Opportunities and challenges for human papillomavirus vaccination in cancer". Nat Rev Cancer 18.4 (2018), 240-254.

[37] F. Carrat and A. Flahault. "Influenza vaccine: the challenge of antigenic drift". Vaccine 25.39-40 (2007), 6852-6862.

[38] P. M. B. English. "Eradicating cervical cancer is unlikely". BMJ 366 (2019), 14953. 
[39] K. U. Jansen, C. Knirsch, and A. S. Anderson. "The role of vaccines in preventing bacterial antimicrobial resistance". Nat Med 24.1 (2018), 10-19.

[40] I. K. Damon. "Status of human monkeypox: clinical disease, epidemiology and research". Vaccine 29 (2011), D54-D59.

[41] D. Bhavnani, J. E. Goldstick, W. Cevallos, et al. "Synergistic effects between rotavirus and coinfecting pathogens on diarrheal disease: evidence from a community-based study in northwestern Ecuador". Am J Epidemiol 176.5 (2012), 387-395.

[42] M. Kretzschmar, M. G. M. Gomes, R. A. Coutinho, et al. "Unlocking pathogen genotyping information for public health by mathematical modeling". Trends in microbiology 18.9 (2010), 406-412.

[43] L. F. Xi, Z. R. Edelstein, C. Meyers, et al. "Human papillomavirus types 16 and 18 DNA load in relation to coexistence of other types, particularly those in the same species". Cancer Epidemiol Biomarkers Prev 18.9 (2009), 2507-2512.

[44] J. Biryukov and C. Meyers. "Superinfection exclusion between two high-risk human papillomavirus types during a coinfection". J Virol 92.8 (2018), e01993-17.

[45] J. E. Tota, A. V. Ramanakumar, M. Jiang, et al. "Epidemiologic approaches to evaluating the potential for human papillomavirus type replacement postvaccination". Am J Epidemiol 178.4 (2013), 625-634.

[46] N. Mejlhede, B. V. Pedersen, M. Frisch, et al. "Multiple human papilloma virus types in cervical infections: competition or synergy?" APMIS 118.5 (2010), 346-352.

[47] S. Vaccarella, S. Franceschi, R. Herrero, et al. "Clustering of multiple human papillomavirus infections in women from a population-based study in Guanacaste, Costa Rica". J Infect Dis 204.3 (2011), 385-390.

[48] K. Auranen, J. Mehtälä, A. Tanskanen, et al. "Between-strain competition in acquisition and clearance of pneumococcal carriage - epidemiologic evidence from a longitudinal study of day-care children". Am J Epidemiol 171.2 (2010), 169-176.

[49] M. Lipsitch, O. Abdullahi, A. D'Amour, et al. "Estimating rates of carriage acquisition and clearance and competitive ability for pneumococcal serotypes in Kenya with a Markov transition model". Epidemiology 23.4 (2012), 510-519.

[50] P. K. Andersen and N. Keiding. "Multi-state models for event history analysis". Stat Methods Med Res 11.2 (2002), 91-115.

[51] S. Højsgaard, D. Edwards, and S. Lauritzen. Graphical models with R. Springer Science \& Business Media, 2012.

[52] C. D. Van Borkulo, D. Borsboom, S. Epskamp, et al. "A new method for constructing networks from binary data". Sci Rep 4.1 (2014), 1-10.

[53] S. L. Ranjeva, E. B. Baskerville, V. Dukic, et al. "Recurring infection with ecologically distinct HPV types can explain high prevalence and diversity". Proc Natl Acad Sci U S A 114.51 (2017), 13573-13578.

[54] D. P. Durham, E. M. Poolman, Y. Ibuka, et al. "Reevaluation of epidemiological data demonstrates that it is consistent with cross-immunity among human papillomavirus types". $J$ Infect Dis 206.8 (2012), 1291-1298.

[55] C. L. Murall, K. S. McCann, and C. T. Bauch. "Revising ecological assumptions about human papillomavirus interactions and type replacement". J Theor Biol 350 (2014), 98-109.

[56] T. Malagón, P. Lemieux-Mellouki, J. F. Laprise, et al. "Bias due to correlation between times-atrisk for infection in epidemiologic studies measuring biological interactions between sexually transmitted infections: a case study using human papillomavirus type interactions". Am J Epidemiol 184.12 (2016), 873-883. 
[57] A. J. Kucharski, V. Andreasen, and J. R. Gog. "Capturing the dynamics of pathogens with many strains". J Math Biol 72.1 (2016), 1-24.

[58] P. S. Wikramaratna, A. Kucharski, S. Gupta, et al. "Five challenges in modelling interacting strain dynamics". Epidemics 10 (2015), 31-34.

[59] M. Plummer, S. Vaccarella, and S. Franceschi. "Multiple human papillomavirus infections: the exception or the rule? (editorial)". J Infect Dis 203.7 (2011), 891-893.

[60] R. McNamee. "Regression modelling and other methods to control confounding". Occup Environ Med 62.7 (2005), 500-506.

[61] M. Drolet, É. Bénard, M. C. Boily, et al. "Population-level impact and herd effects following human papillomavirus vaccination programmes: a systematic review and meta-analysis". Lancet Infect Dis 15.5 (2015), 565-580.

[62] J. E. Tota, F. Struyf, M. Merikukka, et al. "Evaluation of type replacement following HPV16/18 vaccination: pooled analysis of two randomized trials". J Natl Cancer Inst 109.7 (2017), djw300.

[63] P. J. Woestenberg, A. J. King, B. H. Van Benthem, et al. "Bivalent vaccine effectiveness against type-specific HPV positivity: evidence for cross-protection against oncogenic types among Dutch STI clinic visitors". J Infect Dis 217.2 (2018), 213-222.

[64] W. P. Hausdorff and W. P. Hanage. "Interim results of an ecological experiment - Conjugate vaccination against the pneumococcus and serotype replacement". Hum Vaccin Immunother 12.2 (2016), 358-374.

[65] M. Drolet, É. Bénard, N. Pérez, et al. "Population-level impact and herd effects following the introduction of human papillomavirus vaccination programmes: updated systematic review and meta-analysis". Lancet 394.10197 (2019), 497-509.

[66] J. N. Weiser, D. M. Ferreira, and J. C. Paton. "Streptococcus pneumoniae: transmission, colonization and invasion". Nat Rev Microbiol 16.6 (2018), 355-367.

[67] D. S. Fedson and G. Scott. "The burden of pneumococcal disease among adults in developed and developing countries: what is and is not known". Vaccine 17 (1999), S11-S18.

[68] K. L. O'Brien, L. J. Wolfson, J. P. Watt, et al. "Burden of disease caused by Streptococcus pneumoniae in children younger than 5 years: global estimates". Lancet 374.9693 (2009), 893-902.

[69] M. Lipsitch. "Vaccination against colonizing bacteria with multiple serotypes". Proc Natl Acad Sci U S A 94.12 (1997), 6571-6576.

[70] M. Lipsitch. "Bacterial vaccines and serotype replacement: lessons from Haemophilus influenzae and prospects for Streptococcus pneumoniae". Emerg Infect Dis 5.3 (1999), 336-345.

[71] D. M. Weinberger, R. Malley, and M. Lipsitch. "Serotype replacement in disease after pneumococcal vaccination". Lancet 378.9807 (2011), 1962-1973.

[72] J. A. Lewnard and W. P. Hanage. "Making sense of differences in pneumococcal serotype replacement". Lancet Infect Dis 19.6 (2019), e213-e220.

[73] A. Melegaro, Y. Choi, R. Pebody, et al. "Pneumococcal carriage in United Kingdom families: estimating serotype-specific transmission parameters from longitudinal data". Am J Epidemiol 166.2 (2007), 228-235.

[74] J. Mehtälä, M. Antonio, M. S. Kaltoft, et al. "Competition between Streptococcus pneumoniae strains: implications for vaccine-induced replacement in colonization and disease". Epidemiology (2013), 522-529.

[75] D. M. Weinberger, R. Dagan, N. Givon-Lavi, et al. "Epidemiologic evidence for serotype-specific acquired immunity to pneumococcal carriage". J Infect Dis 197.11 (2008), 1511-1518.

[76] K. Trzciński, Y. Li, D. M. Weinberger, et al. "Effect of serotype on pneumococcal competition in a mouse colonization model". mBio 6.5 (2015), e00902-15. 
[77] P. Turner, J. Hinds, C. Turner, et al. "Improved detection of nasopharyngeal cocolonization by multiple pneumococcal serotypes by use of latex agglutination or molecular serotyping by microarray". J Clin Microbiol 49.5 (2011), 1784-1789.

[78] C. Satzke, E. M. Dunne, B. D. Porter, et al. "The PneuCarriage project: a multi-centre comparative study to identify the best serotyping methods for examining pneumococcal carriage in vaccine evaluation studies". PLoS Med 12.11 (2015), e1001903.

[79] C. P. Olwagen, P. V. Adrian, and S. A. Madhi. "Comparison of traditional culture and molecular qPCR for detection of simultaneous carriage of multiple pneumococcal serotypes in African children". Sci Rep 7.1 (2017), 4628.

[80] S. Flasche, O. L. P. de Waroux, K. L. O'Brien, et al. "The serotype distribution among healthy carriers before vaccination is essential for predicting the impact of pneumococcal conjugate vaccine on invasive disease". PLoS Comput Biol 11.4 (2015), e1004173.

[81] D. M. Weinberger, J. L. Warren, T. Dalby, et al. "Differences in the impact of pneumococcal serotype replacement in individuals with and without underlying medical conditions". Clin Infect Dis 69.1 (2019), 100-106.

[82] M. Nurhonen and K. Auranen. "Optimal serotype compositions for pneumococcal conjugate vaccination under serotype replacement". PLoS Comput Biol 10.2 (2014), e1003477.

[83] G. M. Knight, N. G. Davies, C. Colijn, et al. "Mathematical modelling for antibiotic resistance control policy: do we know enough?" BMC Infect Dis 19.1 (2019), 1011.

[84] C. Colijn, J. Corander, and N. J. Croucher. "Designing ecologically optimized pneumococcal vaccines using population genomics". Nat Microbiol (2020), 473-485.

[85] M. Schiffman and P. E. Castle. "Human papillomavirus: epidemiology and public health". Arch Pathol Lab Med 127.8 (2003), 930-934.

[86] "IARC monographs on the evaluation of carcinogenic risks to humans. Human papillomaviruses". IARC Monogr Eval Carcinog Risks Hum 90 (2007).

[87] E. M. A. (EMA). Gardasil: EPAR - Product Information. Accessed: 20-10-2020. URL: https : //www.ema.europa.eu/en/medicines/human/EPAR/gardasil

[88] E. M. A. (EMA). Cervarix: EPAR - Product Information. Accessed: 20-10-2020. URL: https : //www.ema.europa.eu/en/medicines/human/EPAR/cervarix

[89] E. M. A. (EMA). Gardasil 9: EPAR - Product Information. Accessed: 20-10-2020. URL: https : //www.ema.europa.eu/en/medicines/human/EPAR/gardasil-9

[90] S. M. Garland, M. Steben, H. L. Sings, et al. "Natural history of genital warts: analysis of the placebo arm of 2 randomized phase III trials of a quadrivalent human papillomavirus (types 6 , 11, 16, and 18) vaccine". J Infect Dis 199.6 (2009), 805-814.

[91] M. Lehtinen and J. Paavonen. "Vaccination against human papillomaviruses shows great promise". Lancet 364.9447 (2004), 1731-1732.

[92] C. B. Woodman, S. I. Collins, and L. S. Young. "The natural history of cervical HPV infection: unresolved issues". Nat Rev Cancer 7.1 (2007), 11-22.

[93] S. Vaccarella, M. Plummer, S. Franceschi, et al. "Clustering of human papillomavirus (HPV) types in the male genital tract: the HPV in men (HIM) study". J Infect Dis 204.10 (2011), 15001504.

[94] M. Mollers, H. J. Vriend, M. A. van der Sande, et al. "Population- and type-specific clustering of multiple HPV types across diverse risk populations in the Netherlands". Am J Epidemiol 179.10 (2014), 1236-1246.

[95] M. C. Rousseau, J. S. Pereira, J. C. Prado, et al. "Cervical coinfection with human papillomavirus (HPV) types as a predictor of acquisition and persistence of HPV infection". J Infect Dis 184.12 (2001), 1508-1517. 
[96] F. Méndez, N. Muñoz, H. Posso, et al. "Cervical coinfection with human papillomavirus (HPV) types and possible implications for the prevention of cervical cancer by HPV vaccines". J Infect Dis 192.7 (2005), 1158-1165.

[97] K. K. Thomas, J. P. Hughes, J. M. Kuypers, et al. "Concurrent and sequential acquisition of different genital human papillomavirus types". J Infect Dis 182.4 (2000), 1097-1102.

[98] M. Merikukka, M. Kaasila, P. B. Namujju, et al. "Differences in incidence and co-occurrence of vaccine and nonvaccine human papillomavirus types in Finnish population before human papillomavirus mass vaccination suggest competitive advantage for HPV33". Int J Cancer 128.5 (2011), 1114-1119.

[99] A. K. Chaturvedi, L. Myers, A. F. Hammons, et al. "Prevalence and clustering patterns of human papillomavirus genotypes in multiple infections". Cancer Epidemiol Biomarkers Prev 14.10 (2005), 2439-2445.

[100] D. Mesher, K. Soldan, M. Lehtinen, et al. "Population-level effects of human papillomavirus vaccination programs on infections with nonvaccine genotypes". Emerg Infect Dis 22.10 (2016), $1732-1740$. 


\section{Chapter 2}

\section{Approximate likelihood-based estimation method of multiple-type pathogen interactions: an application to longitudinal pneumococcal carriage data}

This chapter has been submitted as: I. Man, J. A. Bogaards, K. Makwana, K. Trzciński, and K. Auranen. "Approximate likelihood-based estimation method of multiple-type pathogen interactions: an application to longitudinal pneumococcal carriage data". 


\section{Abstract}

While serotypes of Streptococcus pneumoniae are known to compete during colonization in human hosts, our knowledge of how competition occurs is still incomplete. New insights of pneumococcal between-type competition could be generated from carriage data obtained by molecular-based detection methods, which record more complete sets of serotypes involved in co-carriage than when detection is done by culture. Here, we develop a Bayesian estimation method for inferring between-type interactions from longitudinal data recording the presence/absence of the types at discrete observation times. It allows inference from data containing co-carriage of two or more serotypes, which is often the case when pneumococcal presence is determined by molecular-based methods. The computational burden posed by the increased number of types detected in cocarriage is addressed by approximating the likelihood under a multi-state model with the likelihood of only those trajectories with minimum numbers of acquisition and clearance between observation times. The proposed method performed satisfactorily on simulated data. The estimates of the interaction parameters of acquisition and clearance were unbiased in settings with short sampling intervals between observation times. With increasing length of the sampling interval, the estimates of the interaction parameters of acquisition and clearance became more biased, but their ratio remained unbiased. Confounding due to unobserved heterogeneity in exposure could be corrected by including individual-level random effects. In an application to empirical data on pneumococcal carriage in infants, we found new evidence for between-type competition in clearance, although the effect size was small. 


\section{Introduction}

A recurrent theme in research of Streptococcus pneumoniae (the pneumococcus) is whether and how pneumococcal serotypes interact with each other [1-8]. As pneumococcal conjugate vaccination has led to serotype replacement, it is evident that between-serotype competition exists [9]. However, the mechanisms by which different serotypes compete remain poorly understood [10, 11].

The way pneumococcal serotypes interfere with each other during colonization (carriage) in human hosts has often been studied in longitudinal settings [27]. Using multi-state models, it has been possible to infer whether and to what extent colonization with one serotype reduces acquisition or enhances clearance of colonization with other types. In such models, the possible combinations of types with which a host can be simultaneously colonised define the model's states, whereas the transitions between these states represent events of acquisition and clearance. With these definitions, interactions in acquisition and clearance can be quantified in terms of ratios between appropriate transition rates. The interaction in acquisition is quantified by the ratio between the rates of acquiring a new serotype in presence versus absence of other types, whereas the interaction in clearance is quantified by the ratio between the rates of clearing a serotype in presence versus absence of other types. Based on estimates of such rate ratios, various studies have concluded that competition in acquisition is likely [2-6]. By contrast, only a few studies have also found evidence for competition in clearance [4, 7].

Nevertheless, previous findings of between-serotype interactions may have been biased by the suboptimal sensitivity of culture-based methods for detecting pneumococcal presence [6, 7]. While culture-based methods seldom detect cocarriage of more than two types, it is common for molecular-based methods to discover co-carriage of three or more types. Cross-validation of samples using both methods suggests an underdetection by culture-based methods when multiple types are present [12-14]. As a result, estimates of between-type interaction may have been biased, likely in the direction towards stronger competition. Estimates based on molecular-level data may provide a more accurate picture of how serotypes interact.

In principle, inference of between-serotype interactions from molecular-level longitudinal data is not different from analysing culture-level data. It is still possible to define rate ratios of acquisition and clearance as measures of interactions. However, estimation of these measures becomes more computationally demanding when the data only record the states of the underlying dynamics at discrete observation times. For instance, when observing the host in the non-carriage state at one observation time and carrying some serotype(s) at the subsequent observation time, the estimation method has to account for the many possible time 
points at which acquisition(s) could have taken place. Moreover, it is possible for this (or another) serotype to be successively acquired and cleared multiple times between the observation times. Exploration of all possible trajectories of acquisition and clearance compatible with the observed data is hence computationally intensive even with culture-level data. With molecular-level data, the task is even more demanding, as the higher number of serotypes detected in co-carriage implies more complex trajectories. Therefore, in order to infer between-serotype interactions from molecular-level data, new approaches need to be developed to deal with the increased computational burden.

In this paper, we develop an estimation method that allows inference of interactions between pathogen types from longitudinal data containing co-carriage of two or more types, as is often the case when common multi-strain pathogens (e.g. Streptococcus pneumoniae, human papillomavirus, Plasmodium falciparum) are detected with molecular-based methods. The new method tackles the computational task by restricting the likelihood function under a multi-state model to account for the likelihood of only those trajectories with a minimal number of transitions. This chapter is organised as follows. The next section specifies the model for multiple-type pneumococcal colonization dynamics, after which we present an approximation of the likelihood function and embed it in a Bayesian estimation framework. Subsequently, we validate the proposed Bayesian approximate likelihood-based estimation method on simulated data and apply the new method to a molecular-level data set of pneumococcal carriage in infants to investigate between-type competition of pneumococcus. Finally, we conclude and discuss our findings.

\section{A multi-state model of multiple-type pneumococcal colonization dynamics}

We here specify a continuous-time multi-state model for the pneumococcal colonisation dynamics of an arbitrary number of serotypes at the individual level. We assume a Markov model so that the transition rates are constant over time. For the pneumococcal data set we considered in this work, the Markov assumption is justifiable as it consists of infants in their first 18 months of life, during which the build-up of naturally acquired serotype-specific immunity as well as crossprotection across types are limited [15, 16]. For simplicity, we also assume any seasonal effects to be negligible.

The model's state space is the set of possible combinations of a given number $p$ of serotypes. Including the uncolonised state $\emptyset$, there are maximal $2^{p}$ states possible. Models previously used for inferring interactions between pneumococcal serotypes from culture-based data contain no co-carriage states of more than 
serotwo types and hence consist maximally of $1+p(p+1) / 2$ states [2-7]. In this work, we also allow some co-carriage states with more types, which will be further specified in the next section. Transitions between the model states take place when individual serotypes are acquired or cleared. We only allow acquisition and clearance of one type at a time so that the model accommodates only transitions between those pairs of states that differ by one type (Figure 2.1). This does not impose a real restriction, as the probability of multiple transitions occurring simultaneously is arguably negligible.

An uncolonised individual acquires type $j$ at rate $z_{i} \lambda_{j}$, where $\lambda_{j}$ is the per capita baseline acquisition rate of type $j$ and $z_{i}$ an individual-level parameter ("random effect") indicating the level of exposure and/or predisposition to pneumococcal colonization of individual $i$. An individual already colonised with one or more of the other types acquires type $j$ at rate $k z_{i} \lambda_{j}$. Hence, parameter $k$ is the rate ratio for acquiring a type in the presence versus absence of any other types and describes the between-type interaction in acquisition, with $k<1$ denoting competition.

In a singly colonised individual, i.e. in absence of any other types, clearance of type $j$ occurs at a type-specific baseline rate $\mu_{j}$, whereas clearance in presence of other types occurs at rate $h \mu_{j}$. Hence, parameter $h$ is the rate ratio for clearing a type in the presence versus absence of other types, describing the between-type interaction in clearance, with $h>1$ denoting competition.

In summary, for a given individual $i$, the transition rate between any two model states, from $x$ to $y$, is given by

$$
Q_{i}(x, y)= \begin{cases}z_{i} \lambda_{j} & \text { if } x=\emptyset \text { and } y=\{j\}, \\ k z_{i} \lambda_{j} & \text { if } x \neq \emptyset, y=x \cup\{j\} \text { and } j \notin x, \\ \mu_{j} & \text { if } y=\emptyset \text { and } x=\{j\}, \\ h \mu_{j} & \text { if } y \neq \emptyset, x=y \cup\{j\} \text { and } j \notin y, \\ 0 & \text { if otherwise, }\end{cases}
$$

where $\emptyset$ denotes the uncolonised state, $\notin$ not contained by, and $\cup$ the union of the respective sets.

Arranging all transition rates of individual $i$ in a matrix yields a transition rate matrix $Q_{i}$, in which the $(x, y)$ element is the transition rate from state $x$ to state $y$. By convention, each diagonal element of $Q_{i}$ is specified as the additive inverse of the total transition rate out of the corresponding state, i.e. $Q_{i}(x)=-\sum_{y \neq x} Q_{i}(x, y)$. 


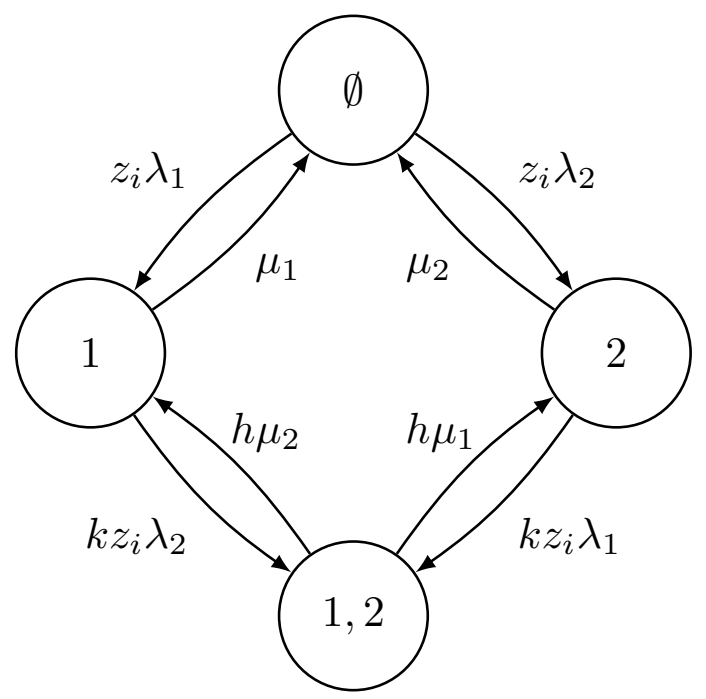

Figure 2.1: Structure of the multi-state model. The depicted multi-state model describes the colonisation dynamics of $p=2$ pneumococcal serotypes at the individual level. This model has $2^{p}=4$ states and $2^{p} \cdot p=8$ transitions. Shown are the baseline acquisition rates, $\lambda_{1}$ and $\lambda_{2}$, the baseline clearance rates, $\mu_{1}$ and $\mu_{2}$, the random effect of individual $i$, $z_{i}$, and the interaction parameters of acquisition and clearance, $k$ and $h$.

\section{A Bayesian approximate likelihood-based estimation method}

Here, we present a Bayesian approximate likelihood-based (BALB) method for estimating the parameters of the described multi-state model when carriage states data $D$ of study subjects have been recorded at discrete observation times. Denote the vector of unknown parameters by $\theta=\left(\lambda_{1}, \cdots, \lambda_{p}, \mu_{1}, \cdots, \mu_{p}, k, h, z_{1}, \cdots, z_{m}\right)^{T}$. For $m$ study subjects, the parameters include $2 p$ baseline rates, 2 interaction parameters and $m$ random effects. The estimation method relies on an approximation of the likelihood function $p(D \mid \theta)$ of the model parameters $\theta$.

Before writing the likelihood function based on the entire data set $D$, we specify how the contribution from one consecutive pair of observations from one individual is approximated. For a moment, for notational convenience, we use $D$ to denote the single pair of observations and $Q$ to denote the transition rate matrix of the individual in question.

Suppose that we observe the individual to be in state $x_{\text {start }}$ at time $t_{\text {start }}$ and in state $x_{\text {end }}$ at the subsequent observation time $t_{\text {end }}$, i.e. $D=\left\{x_{\text {start }}, x_{\text {end }}, t_{\text {start }}, t_{\text {end }}\right\}$. The likelihood contribution $p(D \mid \theta)$ is then given by the total probability of all possible trajectories compatible with this observation, i.e. all paths of connecting states 
starting in state $x_{\text {start }}$ and ending in state $x_{\text {end }}$, with corresponding sojourn times summing up to $\Delta T=t_{\text {end }}-t_{\text {start }}$. Theoretically, this probability can be evaluated by first determining the transition probability matrix $\exp (Q \cdot \Delta T)$ and then taking its $\left(x_{\text {start }}, x_{\text {end }}\right)$ element. However, calculation of the exact transition probability matrix amounts to computing a matrix exponential, which is a computationally intensive task when the dimension of $Q$ is high, i.e. when many states are included in the model [17]. The alternative approach based on data augmentation is also computationally demanding, as a very large number of trajectories needs to be simulated. Instead, we propose to approximate $p(D \mid \theta)$ by restricting to trajectories that contain the minimum number of transitions connecting state $x_{\text {start }}$ to state $x_{\text {end }}$. In other words, we include only those trajectories that contain exactly one transition for each type that is in $x_{\text {start }}$ but not $x_{\text {end }}$, or vice versa, and no transitions for types that are in both $x_{\text {start }}$ and $x_{\text {end }}$, or in neither of them. In effect, when states $x_{\text {start }}$ and $x_{\text {end }}$ differ with regard to the status of $n$ types, a minimumtransition trajectory contains exactly $n$ states and $n-1$ transitions. Note that, due to this restriction to minimum-transition trajectories, it suffices to reduce the model state space to only those co-carriage states that show up in a minimum-transition trajectory.

To enumerate all minimum-transition trajectories, let $\mathcal{X}(D)$ and $\mathcal{T}(D)$ denote the collection of all minimum-transition paths and the collection of all compatible sojourn times, respectively. Suppose that $D=\left\{x_{\text {start }}, x_{\text {end }}, t_{\text {start }}, t_{\text {end }}\right\}=$ $\{\emptyset,\{1,2\}, 0,2\}$. The corresponding collection of paths $\mathcal{X}(D)$ consists of two paths: $(\emptyset,\{1\},\{1,2\})$ and $(\emptyset,\{2\},\{1,2\})$, while the corresponding collection of sojourn times $\mathcal{T}(D)$ consists of all positive triplets summing up to $\Delta T$, e.g. $(0.5,0.5,1.0)$ (Figure 2.2). The approximation of the likelihood contribution $\tilde{p}(D \mid \theta)$ is given by the likelihood of all minimum-transition trajectories, obtained by enumerating all combinations of paths and sojourn times in $\mathcal{X}(D)$ and $\mathcal{T}(D)$ :

$$
\begin{aligned}
& \tilde{p}(D \mid \theta)=\sum_{\boldsymbol{x} \in \mathcal{X}(D)} \int_{\boldsymbol{t} \in \mathcal{T}(D)} p(\boldsymbol{x}, \boldsymbol{t} \mid \theta) d \boldsymbol{t} \\
& =\sum_{\boldsymbol{x} \in \mathcal{X}(D)} \int_{0}^{\Delta T} \int_{0}^{\Delta T-t_{1}} \cdots \int_{0}^{\Delta T-\sum_{r=1}^{n-2} t_{r}} p\left(\boldsymbol{x}, t_{1}, t_{2}, \cdots, t_{n-1}, \Delta T-\sum_{r=1}^{n-2} t_{r} \mid \theta\right) d t_{n-1} \cdots d t_{2} d t_{1} \\
& =\sum_{\boldsymbol{x} \in \mathcal{X}(D)} \int_{0}^{\Delta T} \int_{0}^{\Delta T-t_{1}} \cdots \int_{0}^{\Delta T-\sum_{r=1}^{n-2} t_{r}} e^{Q\left(x_{n}\right)\left(\Delta T-\sum_{r=1}^{n-1} t_{r}\right)} \prod_{r=1}^{n-1} e^{Q\left(x_{r}\right) t_{r}} Q\left(x_{r}, x_{r+1}\right) d t_{n-1} \cdots d t_{2} d t_{1} .
\end{aligned}
$$

The integrand on the last line is the standard likelihood in multi-state models for a single trajectory, in which the exponential terms account for sojourning in the 


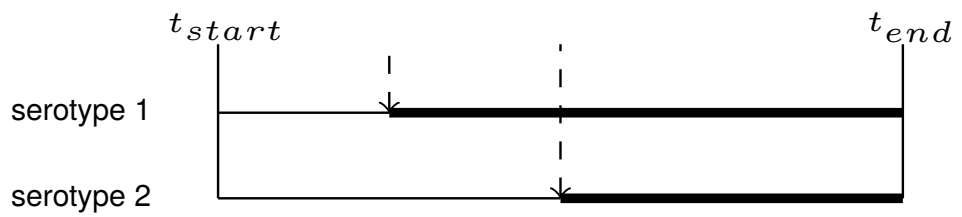

Figure 2.2: Example of a minimum-transition trajectory. The depicted trajectory is one possible minimum-transition trajectory corresponding to data $D=$ $\left\{x_{\text {start }}, x_{\text {end }}, t_{\text {start }}, t_{\text {end }}\right\}=\{\emptyset,\{1,2\}, 0,2\}$. The depicted path is $(\emptyset,\{1\},\{1,2\})$, with sojourn times $(0.5,0.5,1.0)$. Legend: solid vertical lines denote observation times; dashed vertical arrows denote transition times; thin horizontal lines denote non-carriage; thick horizontal lines denote carriage.

visited states and the off-diagonal terms of matrix $Q$ for the respective transitions in between [18].

Equation (2.2) can be further simplified into the following expression, which is less computationally intense to evaluate (see Appendix A for the verification):

$$
\begin{aligned}
& \tilde{p}(D \mid \theta)=\sum_{x \in \mathcal{X}(D)} e^{Q\left(x_{n}\right) \Delta T}\left(\prod_{r=1}^{n-1} Q\left(x_{r}, x_{r+1}\right)\right) . \\
& \quad\left(\frac{(-1)^{n-1}}{\prod_{r=1}^{n-1} Q\left(x_{r}\right)-Q\left(x_{n}\right)}+\sum_{r=1}^{n-1} \frac{e^{\left(Q\left(x_{r}\right)-Q\left(x_{n}\right)\right) \Delta T}}{\left(Q\left(x_{r}\right)-Q\left(x_{n}\right)\right) \prod_{s=1, s \neq r}^{n-1}\left(Q\left(x_{r}\right)-Q\left(x_{s}\right)\right)}\right) .
\end{aligned}
$$

See Appendix B for an analysis demonstrating the goodness of the approximation under reasonable lengths of the sampling interval $\Delta T$.

We now return to the general case in which the data $D$ consist of an arbitrary number of individuals and observation times. An approximation to the entire likelihood is obtained by multiplying the approximate likelihood contributions of all pairs of consecutive observation times, i.e.

$$
\tilde{p}(D \mid \theta)=\prod_{i=1}^{m} \prod_{l=1}^{u_{i}-1} \tilde{p}\left(D_{i l} \mid \theta\right),
$$

where $u_{i}$ is the number of observation times of individual $i$, and $D_{i l}=\left\{x_{l}^{i}, x_{l+1}^{i}, t_{l}^{i}, t_{l+1}^{i}\right\}$ the $l$-th consecutive pair of observations of individual $i$.

Finally, the approximate likelihood function in Equation (2.4) is embedded into a Bayesian framework, in which statistical inference is enabled by estimating the posterior probability of the model parameters according to Bayes' theorem. The posterior probability $p(\theta \mid D)$, which is proportional to the product of the likelihood function $p(D \mid \theta)$ and the prior $p(\theta)$, is approximated through the use of 
the approximate likelihood $\tilde{p}(D \mid \theta)$. The proposed estimation method was implemented in the statistical software STAN (https://github.com/irene-man/), which performs Markov chain Monte Carlo simulation with a Hamiltonian Monte Carlo scheme.

\section{Simulation study}

\section{Methods to compare}

The Bayesian approximate likelihood-based (BALB) estimation method was validated on simulated data. As a benchmark, we compared its performance against a naive method that imputes transition times midway between consecutive observation times. With the imputed transition times, the naive method obtains the maximum likelihood estimates of the model parameters by means of Poisson regression (see Appendix $\mathrm{C}$ for details of the naive method). Note that also the naive method makes the assumption of minimum transitions.

In addition, we investigated how well BALB was able to adjust for bias due to unobserved heterogeneity in exposure and/or predisposition to pneumococcal colonization. To do so, we considered two implementations of BALB: one with individual-specific random effects $\left(z_{i}\right)$ and one without.

\section{Simulated settings}

We simulated longitudinal data sets according to the presented multi-state model. Each data set consisted of 500 individuals with a follow-up period of 20 months. A large number of individuals was chosen to ensure stable estimates, facilitating the identification of biases in the estimated parameter values. For each individual, the initial state at time 0 was non-carriage. Data were simulated assuming the same baseline rates across all serotypes: $\lambda_{j}=\exp (-3.5) \approx 0.030$ per month (acquisition) and $\mu_{j}=\exp (-1.5) \approx 0.22$ per month (clearance). Roughly, in absence of interactions, these rates would yield a prevalence of $12 \%$ for each serotype in the steady state. Parameters that were varied across simulated settings included the number of types $(p)$, length of the sampling interval $(\Delta T)$, interaction parameters $(k$ and $h)$, and variance of the individual-level random effects $z_{i}(\alpha)$.

\section{$B A L B$ and naive methods in absence of heterogeneity in exposure}

We compared the performance of BALB and the naive method in various settings without heterogeneity in exposure. First, setting the number of types to 2, we varied the length of the sampling interval $(\Delta T=0.5,1,2$ months). Then, fixing the sampling interval to 2 months, we varied the number of types $(p=2,4,7)$. For each 
combination of sampling interval and number of types, we explored nine distinct pairs of values for the two interaction parameters, each chosen from $(0.5,1.0,2.0)$. For the interaction parameter of acquisition $k$, these values correspond to competition, independence and synergy, respectively. For the interaction parameter of clearance $h$, they correspond to synergy, independence and competition, respectively.

\section{$B A L B$ under heterogeneity in exposure}

We next investigated whether BALB was able to adjust for unobserved heterogeneity in exposure by including random effects. Heterogeneity was realised by simulating individual-specific random effects $z_{i}$ using a gamma distribution with mean one and variance $\alpha$. We simulated settings with different values of $\alpha$, i.e. $\alpha=0.00001,0.1,0.2,0.5,1$. In the case with the variance as large as 1 , the $20 \%$ of individuals with the largest random effects have at least 7.2 times higher rates for type-specific acquisitions than the $20 \%$ of individuals with the smallest random effects. At the other extreme, $\alpha=0.00001$ resembles the homogeneous case. Throughout, the number of types was fixed at 2 and the length of sampling interval fixed at 1 month. These settings of different values of $\alpha$ were repeated for two combinations of interaction parameters: 1) competition in acquisition and no competition in clearance $(k=0.5, h=1)$ and 2) no competition in acquisition and competition in clearance $(k=1, h=2)$.

\section{Prior distributions}

We assumed the following prior distributions for the model parameters in BALB. The baseline acquisition and clearance rates, $\lambda_{j}$ and $\mu_{j}$, were assumed to have gamma-distributed priors. The corresponding rate parameters were chosen as the crude baseline rates, which were derived from the data (see Appendix D for the derivation). The corresponding shape parameters were fixed to a small value $(0.00001)$ to be non-informative. For the interaction parameters, we assumed uniform priors on the log scale with a symmetrical range around zero to allow as much competitive as synergistic interactions, i.e. $\log (k), \log (h) \sim \operatorname{Unif}(-3,3)$. The random effects were assumed to follow a gamma distribution with mean one and variance $\alpha$ to ensure identifiability, i.e. $z_{i} \sim \operatorname{Gamma}\left(\frac{1}{\alpha}, \frac{1}{\alpha}\right)$, where $\frac{1}{\alpha}$ is both the shape and rate parameter. The random effects $z_{i}$ and the parameter $\alpha$ needed to be estimated from the data in order to mimic the situation in which there is no external information on variation in exposure levels (or susceptibility to colonization) across study participants. For $1 / \alpha$, we assumed the following log-normaldistributed hyperprior: $1 / \alpha \sim \operatorname{Lognormal}(0,2)$. 


\section{Performance measures}

To test the performance of the proposed method, we simulated 100 data sets for each setting, from which the model parameters were estimated. For the Bayesian methods, estimates were given by posterior means and 95\% credible intervals (Cls). For the naive method, estimates were given as maximum likelihood estimates and $95 \%$ confidence intervals (also abbreviated as Cls). To assess bias in the estimation of the baseline rates and the log-transformed interaction parameters, we computed the difference between the true parameter values and the mean of the estimates across the 100 simulated data sets. In addition, we also assessed the bias in the estimates of the log-transformed ratio of the two interaction parameters, $\log (k / h)$.

\section{Results}

Performance of BALB and the naive methods in absence of heterogeneity in exposure

With 2 types and a short sampling interval of 0.5 months, both the BALB and naive methods were able to estimate the interaction parameters with little bias (Figure 2.3A, Table S2.2). With increasing length of the sampling interval, both methods led to biased estimation, except when there was no interaction in either acquisition or clearance (Figure 2.3 A-C, Table S2.2). The interaction parameter in clearance $\log (h)$ was biased towards zero (no interaction), while the interaction parameter in acquisition $\log (k)$ was either over- or under-estimated. Overall, estimates under BALB were less biased than under the naive method. In particular, BALB was better in estimating the ratio of the two interaction parameters $\log (k / h)$ as the sampling interval became longer; in Figure 2.3, the estimates of BALB (green) stayed closer to the dashed slopes containing the true parameter values than the estimates of the naive method (red). When increasing the number of types, the biases under both methods remained at similar levels (Figure 2.3 P-F, Table S2.3). However, the Cls became narrower. In some instances, the Cls even excluded the true parameter values due to the precise but biased estimation.

Regarding the estimation of the baseline rates, again, short sampling intervals (0.5 months) safeguarded against biased estimation; longer sampling intervals induced larger bias, while increasing the number of types did not influence the bias (Tables S2.4 and S2.5). The bias due to the increasing length of sampling interval was comparable across the two methods. Of note, longer sampling intervals led to systematic underestimation of both baseline rates by both methods. Such underestimation is caused by the minimum-transition assumption, used in both the BALB and naive methods. When a complete carriage episode is missed, the true trajectory cannot be captured by a minimum-transition trajectory, as it requires acquisition and clearance of the same type. Both the numbers of acquisition and 

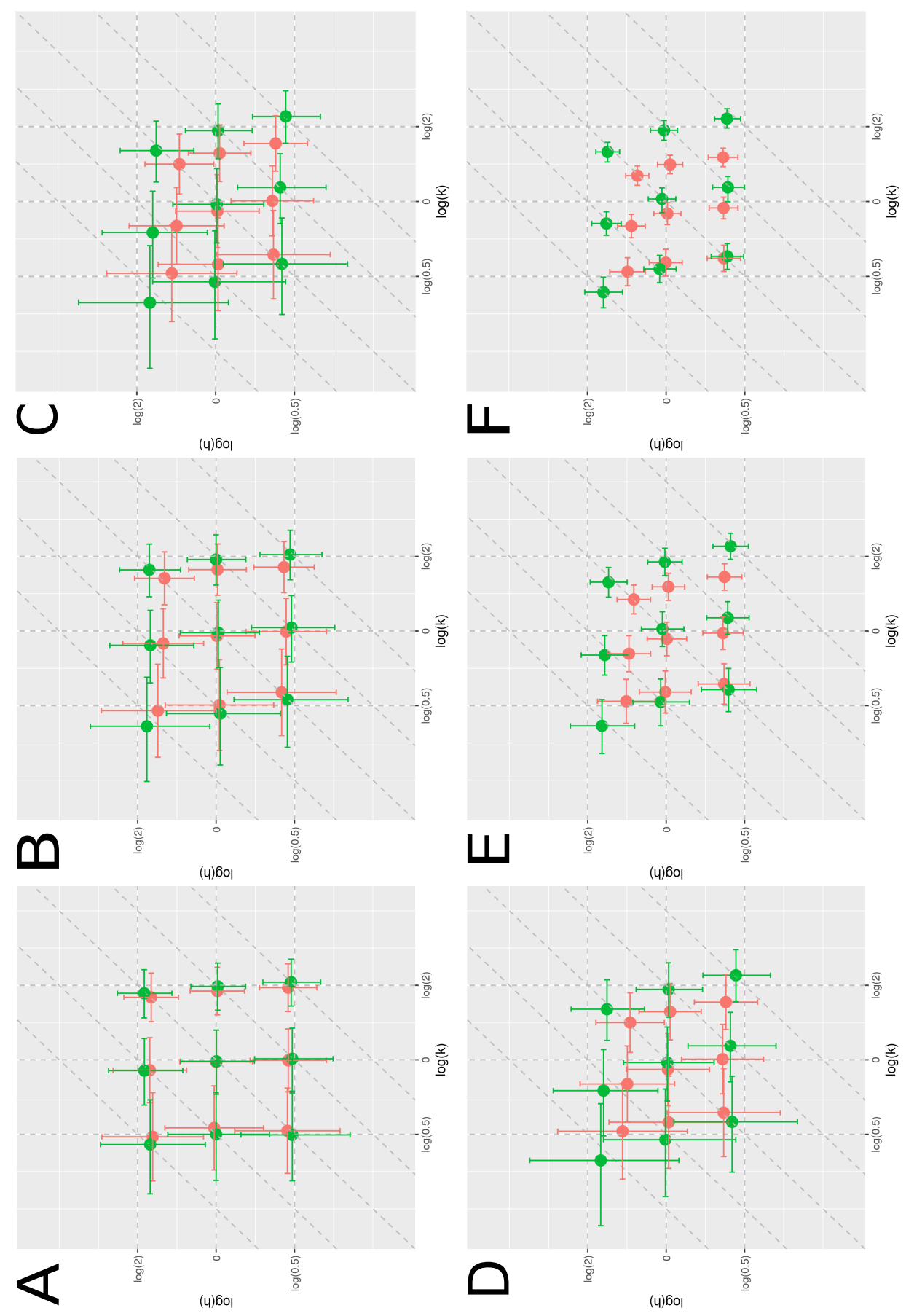

Figure 2.3: Estimates of BALB and the naive method in absence of heterogeneity in exposure. Estimates of the log-transformed interaction parameters, $\log (k)$ and $\log (h)$, obtained by BALB (green) and the naive method (red), in settings in absence of heterogeneity in exposure. The top row shows the settings with 2 types and increasing length of sampling intervals: (A) 0.5 months, (B) 1 month and (C) 2 months. The bottom row shows the settings with 2-month sampling intervals and increasing number of types: (D) 2 types (E) 4 types and (F) 7 types. The vertical and horizontal dashed lines show the true values of $\log (k)$ and $\log (h)$, respectively. The dashed slopes through the intersections show the isoclines of $\log (k / h)$. 
clearance events are then underestimated, which in turn leads to an underestimation of the baseline rates.

\section{Performance of BALB under heterogeneity in exposure}

Next, we explored the ability of BALB to adjust for unobserved heterogeneity in exposure. In the setting with competition in acquisition but no interaction in clearance $(k=0.5, h=1)$, BALB became increasingly biased in estimating the interaction parameter of acquisition with increasing heterogeneity when random effects were not included in the analysis (Figure 2.4A, Table S2.6). The bias was towards stronger synergy; when unobserved heterogeneity was sufficiently large $(\alpha=1)$, competition in acquisition was even erroneously indicated as synergistic interaction. By including random effects, BALB was able to correct for the unobserved heterogeneity. However, in the setting resembling homogeneity, the estimates of interaction in acquisition were slightly more biased towards stronger competition if random effects were allowed, likely due to overfitting. It is also noteworthy that the estimates of the interaction parameter in clearance did not seem to be affected by the amount of heterogeneity, as the heterogeneity acts on the acquisition rates (Figure 2.4B, Table S2.6). The other setting with competition in clearance but no interaction in acquisition ( $k=1, h=2)$ gave the same qualitative results (Figure 2.4C-D, Table S2.6).

\section{Application to longitudinal pneumococcal carriage data}

\section{Data collection}

Using BALB, we then analysed a data set of pneumococcal carriage derived from 45 newborn infants in the Netherlands. Each infant was followed from birth up to at most 18 months of age. Nasopharyngeal samples were collected according to a decelerating sampling scheme in which samples were taken with one-month intervals up to 8 months of age and subsequently at 10,12, 15 and 18 months of age. Occasionally, additional samples were obtained if the infant experienced a respiratory infection. Nine infants were vaccinated with the 7-valent and 36 infants with the 10-valent pnemococcal conjugate vaccine.

Carriage of $S$. pneumoniae and of individual pneumococcal serotypes was determined using both conventional diagnostic culture and molecular methods. The presence of 21 pneumococcal serotypes was determined as follows. For 14 serotypes (3, 6C, 9N, 10A, 11A, 12F, 15A, 15BC, 16F, 19A, 19F, 22F, 23A and $33 F$ ), presence was identified both with molecular-based (qPCR) methods and culture. Carriage of the remaining 7 serotypes (17F, 21, 23B, 25F, 31, 35B and 

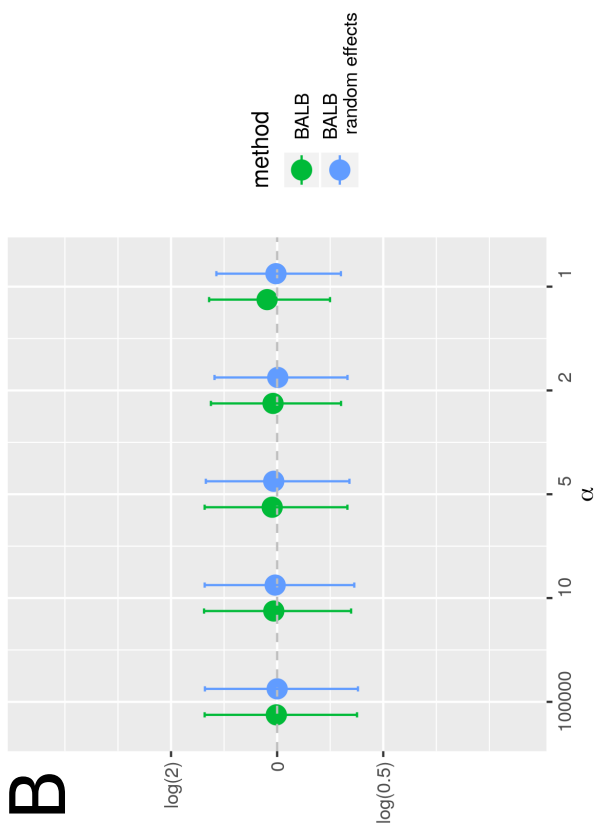

(4) 6 이

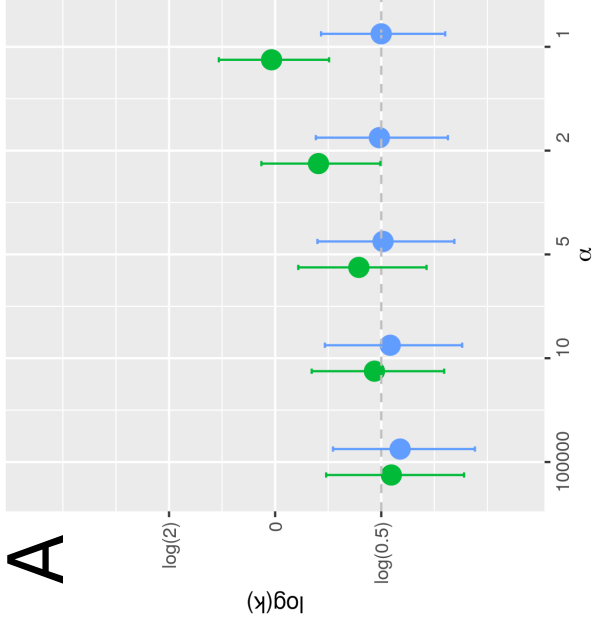

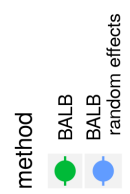

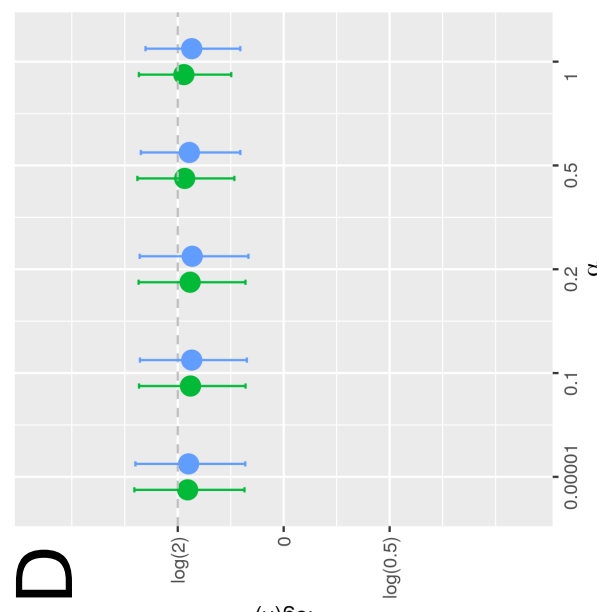

(4)60이

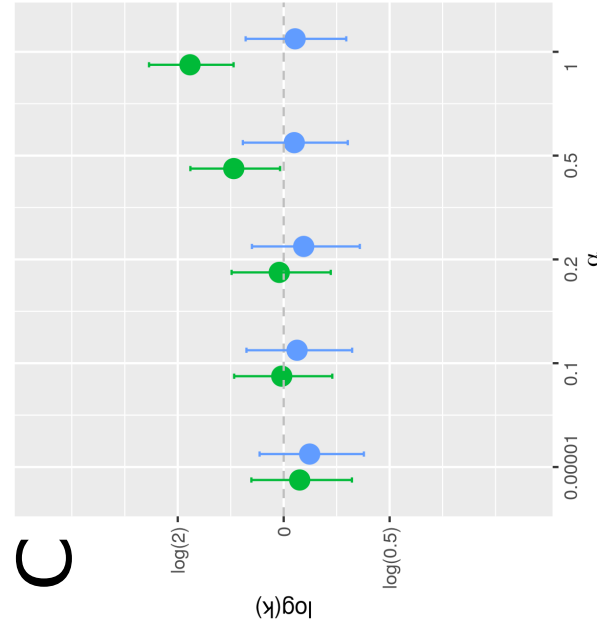

Figure 2.4: Estimates of BALB under increasing heterogeneity in exposure. Estimates of the log-transformed interaction parameters $\log (k)(\mathrm{A}, \mathrm{C})$ and $\log (h)(\mathrm{B}, \mathrm{D})$ obtained by BALB with (blue) and without (green) random effects in settings with 2 types, one-month sampling interval and increasing amount of unobserved heterogeneity in exposure. The top row $(A, B)$ shows the settings with competition in acquisition but no competition in clearance $(k=0.5, h=1)$. The bottom row $(\mathrm{C}, \mathrm{D})$ shows the settings with no competition in acquisition but competition in clearance $(k=1, h=2)$. The horizontal dashed lines show the true values of $\log (k)$ (left) and $\log (h)$ (right). 
35F) was identified by culture only because no molecular-based method was available at the time of data collection. Positivity was defined as positive by either one of the two methods. Samples positive for $S$. pneumoniae for which the serotype could not be determined were assigned as non-typable (NT). Details of the detection methods are given elsewhere [19].

The data set comprises 538 samples, of which 109 (20\% of 538) were additional samples obtained due to respiratory infections. Co-carriage of up to 4 serotypes was detected. In total, 335 (62\% of 538 ) of the samples were positive for at least one serotype, among which 107 (32\% of 335) were positive for multiple serotypes (see Figure S2.1 for the distribution of the number of types in co-carriage in the data). Pneumococcal carriage was heterogeneous across the serotypes, ranging from $2.6 \%$ to $12.3 \%$ of the samples (Figure S2.2). The proportion of samples positive for $S$. pneumoniae also varied across individuals, which was $43.5 \%$ among the $50 \%$ of the study participants with the least carriage and $79.5 \%$ among the $50 \%$ of the study participants with the most carriage. This suggests the need to adjust for heterogeneity in exposure (or predisposition to pneumococcal colonization) across individuals using random effects.

\section{Methods}

We analysed the pneumococcal data set with the naive method and with the BALB method, with and without random effects. To ensure stable estimates, we assumed common baseline acquisition and clearance rates for the seven rarest serotypes (12F, 17F, 22F, 25F, 3, 31 and $9 \mathrm{~N})$ in all methods. For BALB, the same priors for the model parameters were used as in the simulation study, except for $\alpha$, for which a half-normal distribution was assumed. For the half-normal prior distribution around zero, we also explored the effect of increasing standard deviation $(\mathrm{sd}=0.01,0.1,1,10)$.

\section{Results}

The baseline rates estimated by the different methods were comparable, whereas the estimates of the interaction parameters were quite divergent (Figure 2.5, Table S2.8). While the naive method indicated competition in acquisition and synergy in clearance, BALB with or without random effects suggested competition in clearance and synergy in acquisition. Similar to the simulation study, using wider priors for $\alpha$ in BALB with random effects led to smaller estimates of the interaction parameter of acquisition, corresponding to less synergy (or more competition). Simultaneously, the estimates of the interaction parameters of clearance became slightly smaller, corresponding to less competition.

The estimates of the log-transformed ratio of the two interaction parameters $\log (k / h)$ were more consistent across the different methods. The naive method 
yielded a large negative log-transformed ratio, indicating strong competition. The estimates of BALB were closer to zero. BALB without random effects found a small but positive log-transformed ratio. Allowing more heterogeneity across individuals turned the estimated log-transformed ratio to a negative value, indicating competition.

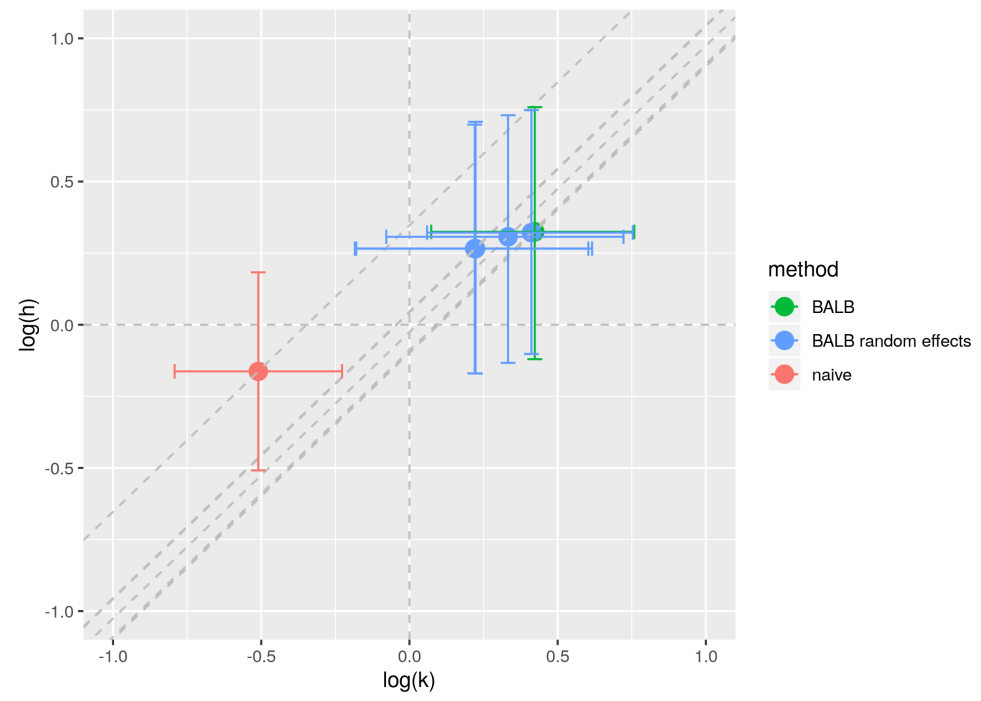

Figure 2.5: Estimates of BALB and the naive method for the pneumococcal application. Estimates of the $\log$-transformed interaction parameters $\log (k)$ and $\log (h)$ obtained by the naive method (red), BALB with (blue) and without (green) random effects. Using wider priors for the variance $\alpha$ of the random effects by increasing the standard deviation of its half-normal prior from $0.01,0.1,1$ to 10 resulted in smaller estimates of the interaction parameter of acquisition (blue circles more to the left).

\section{Discussion}

In this paper, we developed a new estimation method for inferring interactions between multiple types of the same pathogen from longitudinal data. The new method was developed to accommodate inference of between-serotype interactions from pneumococcal carriage data obtained using molecular-based detection methods, typically yielding increased levels of co-carriage as compared to traditional culture-based methods. Multi-state models have difficulty in dealing with co-carriage states of more than two types due to the increased computational burden of exploring all possible acquisition and clearance events between discrete observation times. The new estimation method tackles the computational task by approximating the likelihood function of the parameters in a multi-state model 
with the likelihood of trajectories with a minimum number of transitions. The approximate likelihood function was embedded in a Bayesian framework and implemented according to a computationally efficient Hamiltonian Monte Carlo scheme. The performance of the resulting Bayesian approximate likelihood-based (BALB) estimation method was demonstrated in a simulation study. Moreover, by applying BALB to a pneumococcal carriage data set, we were able to shed new light on the extent and mechanism by which pneumococcal serotypes compete during colonization.

Because the computational burden of inferring between-type interactions could already be high for models with only co-carriage states up to two types, many previous methods have also taken recourse to some form of approximation to mitigate this burden. For example, estimation methods based on data augmentation or maximum likelihood estimation have also relied on the minimum-transition assumption [4, 6]. There are some that relax this assumption, but all previously considered multi-state models omit co-carriage states of more than two serotypes [2, 3, 5, 7, 20]. By capping the number of co-occurring serotypes to two, it is implicitly assumed that an individual already colonised with two types may not acquire any additional types before some are cleared first, which may induce a bias towards stronger competition.

We evaluated the performance of BALB using simulated data. As a benchmark, we used a naive estimation method that simply imputes transition times midway between the observation times. Although the simple method is computationally undemanding, it may be more prone to bias as midpoint transitions may systematically shorten or lengthen episodes of carriage or non-carriage relative to the true colonization history. Indeed, BALB produced more consistent estimates than the naive method in the simulation study.

The simulation study also revealed under which circumstances BALB may perform suboptimally. Long sampling intervals led to biases in the estimates of the model parameters: the baseline rates were underestimated; interaction in clearance was biased towards no competition; however, interaction in acquisition could be either over- or underestimated. Nevertheless, the ratio of the two interaction parameters remained relatively unbiased even with long sampling intervals. The underestimation of baseline rates is due to the missing of a portion of complete carriage episodes between consecutive observation times, which cannot be accounted for by the minimum-transition assumption. It is less clear what determines the direction of biases in the interaction parameters. Our hypothesis is that the rates of clearance are more easily biased than the rates of acquisition so that estimation of interaction in clearance also becomes more difficult. While the average duration of a carriage episode is around the same order of magnitude as the sampling interval, periods of non-carriage are much longer, facilitating precise estimation of acquisition rates. As the ratio of the two interaction parameters is more easily identifiable, it is conceivable that the method tends to compensate 
for the bias in the interaction parameter of clearance with the interaction parameter of acquisition. More investigation is needed to substantiate this hypothesis. While it would be ideal to estimate all parameters without bias, it could be sufficient to accurately estimate the ratio of the interaction parameters in situations where the total amount of competition is more relevant. As we have shown previously, the ratio of the interaction parameters in acquisition and clearance constitutes a natural summary measure of the interactions in these two modes and contains a predictive value for serotype replacement [7, 21].

The simulation study also showed that BALB was capable of adjusting for unobserved heterogeneity in exposure or predisposition to pneumococcal colonization by including individual-specific random effects. In the context of the motivating data on pneumococcal carriage in infants, exposure of pneumococcal carriage may be heterogeneous due to differences in, for example, the number of siblings and attendance to daycare centres. When unadjusted for, unobserved factors that increase the risk of carriage irrespective of type could induce positive associations between different serotypes, masking possible competition between serotypes [21]. In the simulation study, adjustment for unobserved heterogeneity by including random effects worked satisfactorily. However, it could be more challenging in reality. Possible challenges include correct specification of the distribution of random effects and the mechanism through which unobserved heterogeneity acts. Therefore, measuring and modelling possible confounders remain pivotal.

After validation on simulated data, the proposed estimation method was used to study interactions between pneumococcal serotypes based on a data set of pneumococcal carriage obtained using molecular-based detection methods. In line with previous results, which were all derived from culture-based data [1, 2, 4 6. 7], we found evidence for competition between serotypes. Since the detection method that we used to determine the composition of co-carrying serotypes in samples is more sensitive than the culture-based methods used in previous studies, we anticipated the competition to be weaker than previously estimated [13]. Nevertheless, the estimated effect size is surprisingly low. An additional explanation is that the serotypes with strong competitive ability were already removed from the vaccinated study population, as the vaccine targets serotypes that dominated in carriage, such as $6 \mathrm{~B}, 14,19 \mathrm{~F}$ and $23 \mathrm{~F}$, which are arguably also the strong competitors [9, 22, 23]. Also surprising is that the competition was found in clearance and not in acquisition in this data set. Although previous carriage studies have identified competition in acquisition to be the main mechanism, some of them as well as experiments in mouse models have suggested competition in clearance [4, 7, 8]. Analysis of other data sets is needed to validate these results.

The analysis of between-type interactions on the pneumococcal data set has various shortcomings. Most importantly, the sample size was small (45 individuals). To put this number into perspective, most other longitudinal carriage data 
sets used for estimating pneumococcal interactions have consisted of more than a hundred subjects [2,-7, 20]. In addition, at the time of data collection, only 15 of the 21 serotypes could be identified by molecular-based methods. The 6 remaining serotypes were thus detected using culture-based methods only. The level of unobserved heterogeneity may also have complicated identification of between-type interactions. The inclusion of random effects may have only been able to partly account for this. Adjustment based on the collected background information of the study participants and seasonal effects could have further strengthened the analysis.

In the future, the proposed method could be applied to other data sets to further elucidate the interaction between pneumococcal serotypes. In principle, the approach remains valid as long as the transition rates are constant between observation times. For instance, it is possible to model type-specific interaction parameters, which may enable detection of possible differences in competitive ability across pneumococcal serotypes, a topic that has only been touched upon in previous studies [2, 6, 7]. When factors such as age, season and medication use may be relevant to pneumococcal carriage [6, 20], they could be included as covariates. While introducing more parameters may make the model more realistic, it should be done with caution, as identification of a large number of parameters is challenging, especially when the amount of data is limited.

As methods to detect multi-strain pathogens will continue to improve and become more accessible, we expect more opportunities to study the interactions between pneumococcal serotypes or strains of other pathogens, such as the human papillomavirus and Plasmodium falciparum [24, 25]. Existing statistical and computational methods may need to be modified to accommodate analysis of the resulting higher-resolution data as was done in this work. In turn, new knowledge of between-strain interactions may help to better understand and improve the impact of key preventive and therapeutic interventions (e.g. vaccination and antibiotics) against multi-strain pathogens [10, 26].

\section{References}

[1] M. Lipsitch, J. K. Dykes, S. E. Johnson, et al. "Competition among Streptococcus pneumoniae for intranasal colonization in a mouse model". Vaccine 18.25 (2000), 2895-2901.

[2] A. Melegaro, Y. Choi, R. Pebody, et al. "Pneumococcal carriage in United Kingdom families: estimating serotype-specific transmission parameters from longitudinal data". Am J Epidemiol 166.2 (2007), 228-235.

[3] F. Hoti, P. Erästö, T. Leino, et al. "Outbreaks of Streptococcus pneumoniae carriage in day care cohorts in Finland - implications for elimination of transmission". BMC Infect Dis 9 (2009), 102.

[4] K. Auranen, J. Mehtälä, A. Tanskanen, et al. "Between-strain competition in acquisition and clearance of pneumococcal carriage - epidemiologic evidence from a longitudinal study of day-care children". Am J Epidemiol 171.2 (2010), 169-176. 
[5] P. Erästö, F. Hoti, and K. Auranen. "Modeling transmission of multitype infectious agents: application to carriage of Streptococcus pneumoniae". Stat Med 31.14 (2012), 1450-1463.

[6] M. Lipsitch, O. Abdullahi, A. D'Amour, et al. "Estimating rates of carriage acquisition and clearance and competitive ability for pneumococcal serotypes in Kenya with a Markov transition model". Epidemiology 23.4 (2012), 510-519.

[7] J. Mehtälä, M. Antonio, M. S. Kaltoft, et al. "Competition between Streptococcus pneumoniae strains: implications for vaccine-induced replacement in colonization and disease". Epidemiology (2013), 522-529.

[8] K. Trzciński, Y. Li, D. M. Weinberger, et al. "Effect of serotype on pneumococcal competition in a mouse colonization model". mBio 6.5 (2015), e00902-15.

[9] D. M. Weinberger, R. Malley, and M. Lipsitch. "Serotype replacement in disease after pneumococcal vaccination". Lancet 378.9807 (2011), 1962-1973.

[10] W. P. Hausdorff and W. P. Hanage. "Interim results of an ecological experiment - Conjugate vaccination against the pneumococcus and serotype replacement". Hum Vaccin Immunother 12.2 (2016), 358-374.

[11] J. A. Lewnard and W. P. Hanage. "Making sense of differences in pneumococcal serotype replacement". Lancet Infect Dis 19.6 (2019), e213-e220.

[12] P. Turner, J. Hinds, C. Turner, et al. "Improved detection of nasopharyngeal cocolonization by multiple pneumococcal serotypes by use of latex agglutination or molecular serotyping by microarray". J Clin Microbiol 49.5 (2011), 1784-1789.

[13] C. Satzke, E. M. Dunne, B. D. Porter, et al. "The PneuCarriage project: a multi-centre comparative study to identify the best serotyping methods for examining pneumococcal carriage in vaccine evaluation studies". PLoS Med 12.11 (2015), e1001903.

[14] C. P. Olwagen, P. V. Adrian, and S. A. Madhi. "Comparison of traditional culture and molecular qPCR for detection of simultaneous carriage of multiple pneumococcal serotypes in African children". Sci Rep 7.1 (2017), 4628.

[15] D. M. Weinberger, R. Dagan, N. Givon-Lavi, et al. "Epidemiologic evidence for serotype-specific acquired immunity to pneumococcal carriage". J Infect Dis 197.11 (2008), 1511-1518.

[16] S. M. Granat, J. Ollgren, E. Herva, et al. "Epidemiological evidence for serotype-independent acquired immunity to pneumococcal carriage". J Infect Dis 200.1 (2009), 99-106.

[17] C. Moler and C. Van Loan. "Nineteen dubious ways to compute the exponential of a matrix". SIAM review 20.4 (1978), 801-836.

[18] P. K. Andersen and N. Keiding. "Multi-state models for event history analysis". Stat Methods Med Res 11.2 (2002), 91-115.

[19] A. Wyllie, D. Bogaert, S. Prevaes, et al. "Carriage of Streptococcus pneumoniae in the first 18 months of life". Manuscript in preparation ().

[20] E. Numminen, C. Chewapreecha, C. Turner, et al. "Climate induces seasonality in pneumococcal transmission". Sci Rep 5 (2015), 11344.

[21] I. Man, J. Wallinga, and J. A. Bogaards. "Inferring pathogen type interactions using crosssectional prevalence data: opportunities and pitfalls for predicting type replacement". Epidemiology 29.5 (2018), 666-674.

[22] D. M. Weinberger, K. Trzciński, Y.-J. Lu, et al. "Pneumococcal capsular polysaccharide structure predicts serotype prevalence". PLoS Pathog 5.6 (2009), e1000476.

[23] M. Vissers, A. J. Wijmenga-Monsuur, M. J. Knol, et al. "Increased carriage of non-vaccine serotypes with low invasive disease potential four years after switching to the 10-valent pneumococcal conjugate vaccine in The Netherlands". PLoS One 13.3 (2018), e0194823. 
[24] S. L. Ranjeva, E. B. Baskerville, V. Dukic, et al. "Recurring infection with ecologically distinct HPV types can explain high prevalence and diversity". Proc Natl Acad Sci U S A 114.51 (2017), 13573-13578.

[25] A. Lerch, C. Koepfli, N. E. Hofmann, et al. "Longitudinal tracking and quantification of individual Plasmodium falciparum clones in complex infections". Sci Rep 9.1 (2019), 3333.

[26] C. Colijn, J. Corander, and N. J. Croucher. "Designing ecologically optimized pneumococcal vaccines using population genomics". Nat Microbiol (2020), 473-485. 


\section{Appendices of Chapter 2}

\section{A. Analytical expression of the approximate likelihood}

In this appendix, we show the equivalence of the last line of Equation (2.2) to the analytical expression given by Equation (2.3) i.e.

$$
\begin{aligned}
& \sum_{\boldsymbol{x} \in \mathcal{X}(D)} \int_{0}^{\Delta T} \int_{0}^{\Delta T-t_{1}} \cdots \int_{0}^{\Delta T-\sum_{r=1}^{n-2} t_{r}} e^{Q\left(x_{n}\right)\left(\Delta T-\sum_{r=1}^{n-1} t_{r}\right)} \prod_{r=1}^{n-1} e^{Q\left(x_{r}\right) t_{r}} Q\left(x_{r}, x_{r+1}\right) d t_{n-1} \cdots d t_{2} d t_{1} \\
& =\sum_{\boldsymbol{x} \in \mathcal{X}(D)} e^{Q\left(x_{n}\right) \Delta T}\left(\prod_{r=1}^{n-1} Q\left(x_{r}, x_{r+1}\right)\right) \\
& \cdot\left(\frac{(-1)^{n-1}}{\prod_{r=1}^{n-1} Q\left(x_{r}\right)-Q\left(x_{n}\right)}+\sum_{r=1}^{n-1} \frac{e^{\left(Q\left(x_{r}\right)-Q\left(x_{n}\right)\right) \Delta T}}{\left(Q\left(x_{r}\right)-Q\left(x_{n}\right)\right) \prod_{s=1, s \neq r}^{n-1}\left(Q\left(x_{r}\right)-Q\left(x_{s}\right)\right)}\right)
\end{aligned}
$$

First, we bring $e^{Q\left(x_{n}\right) \Delta T}$ and $\prod_{r=1}^{n-1} Q\left(x_{r}, x_{r+1}\right)$ out of the nested integral in the left-hand side of the equation. As these terms are also present in the right-hand side of the equation, we only still have to check whether the rest of the expressions on both sides are equal. We checked this using Wolfram Mathematica with the following code for $n=2,3,4,5$. In the following piece of code, pi represents $Q\left(x_{i}\right)-Q\left(x_{n}\right)$. Verification up to $n=5$ is justifiable in the motivating data set, as the data record at most differences in 4 serotypes between consecutive observation times. A formal proof of this analytical expression for any integer number $n$ may require some induction argument.

(*Define the nested integrals*)

$\mathrm{d} 2=$ Integrate $[\operatorname{Exp}[\mathrm{p} 1 \mathrm{t} 1],\{\mathrm{t} 1,0, \mathrm{te}\}]$;

$\mathrm{d} 3=$ Integrate [Integrate $[\operatorname{Exp}[\mathrm{p} 1 \mathrm{t} 1+\mathrm{p} 2 \mathrm{t} 2],\{\mathrm{t} 2,0, \mathrm{te}-\mathrm{t} 1\}],\{\mathrm{t} 1,0, \mathrm{te}\}] ;$

$\mathrm{d} 4=$ Integrate [Integrate [Integrate [Exp[p1 t1+p2 t2+p3 t3], \{t3,0,te-t1-t2\}], \{t2, 0,te-t1\}],

$\{\mathrm{t} 1,0, \mathrm{te}\}]$;

$\mathrm{d} 5=$ Integrate [Integrate [Integrate [Integrate [Exp [p1 t1+p2 t2+p3 t3+p4t $t$ ] ,

$\{t 4,0, t e-t 1-t 2-t 3\}],\{t 3,0, t e-t 1-t 2\}],\{t 2,0, t e-t 1\}],\{t 1,0, t e\}] ;$

(*Define the analytical expression*)

$\mathrm{P}=\{\mathrm{p} 1, \mathrm{p} 2, \mathrm{p} 3, \mathrm{p} 4\}$;

$d\left[n_{-}\right]:=P l u s[$ Times [Power $[-1, \operatorname{Plus}[-1, n]]$, Power [Product $\left.\left.[\operatorname{Part}[\mathrm{P}, \mathrm{r}], \operatorname{List}[\mathrm{r}, 1, \mathrm{P}] \mathrm{us}[-1, \mathrm{n}]]],-1\right]\right]$,

Sum [Times [Power [E, Times [te, Part [P, r] ] ] , Power [Part [P, r] , -1] , Power [Product [Plus [Part [P, r] ,

Times $[-1, \operatorname{Part}[\mathrm{P}, \mathrm{s}]]]$, List $[\mathrm{s}, 1, \mathrm{Plus}[-1, \mathrm{r}]]],-1]$, Power [Product $[\mathrm{Plus}[\mathrm{Part}[\mathrm{P}, \mathrm{r}]$,

Times $[-1, \operatorname{Part}[\mathrm{P}, \mathrm{s}]]]$, List $[\mathrm{s}, \operatorname{Plus}[1, \mathrm{r}], \operatorname{Plus}[-1, \mathrm{n}]]],-1]], \operatorname{List}[\mathrm{r}, 1, \mathrm{Plus}[-1, \mathrm{n}]]]]$;

(*Check whether the nested integrals are the same as the analytical expression*)

FullSimplify [d[2] ] ===FullSimplify [d2]

FullSimplify $[d[3]]===F u l l$ Simplify $[\mathrm{d} 3]$

FullSimplify $[d[4]]===F u l l$ Simplify $[d 4]$

FullSimplify $[\mathrm{d}[5]]===$ FullSimplify $[\mathrm{d} 5]$

\section{B. Justification of the minimum-transition assumption}

In this appendix, we investigate how well Equation (2.3) of the main text approximates the exact likelihood of the model parameters for different lengths of the sampling interval. This is done in models with a limited number of types and states so that the computation of the exact likelihood is still feasible. The performance of the approximation is then extrapolated to settings with more types. 
The exact likelihood function can be derived from the transition probability matrix, which is given by $\exp (\Delta T \cdot Q)$. Specifically, the likelihood of observing a pair of states at two consecutive observation times, $x$ and $y$, is given by the $(x, y)$ element of $\exp (\Delta T \cdot Q)$. As a given data set may contain all possible combinations of pairs of states, we compare all elements of $\exp (\Delta T \cdot Q)$ to the corresponding approximation as given by Equation (2.3)

As an example, consider the multi-state model as described in the main text with two types and the following model parameters: $k=0.5, h=1.25, \lambda_{1}=0.02, \lambda_{2}=0.03, \mu_{1}=0.1, \mu_{2}=0.2$ per month. The corresponding transition rate matrix of an individual with unity random effect then becomes

$$
\begin{aligned}
& Q=\left[\begin{array}{cccc}
-\left(\lambda_{1}+\lambda_{2}\right) & \lambda_{1} & \lambda_{2} & 0 \\
\mu_{1} & -\left(\mu_{1}+k \lambda_{2}\right) & 0 & k \lambda_{2} \\
\mu_{2} & 0 & -\left(\mu_{2}+k \lambda_{1}\right) & k \lambda_{1} \\
0 & h \mu_{2} & h \mu_{1} & -\left(h \mu_{1}+h \mu_{2}\right)
\end{array}\right] \\
& =\left[\begin{array}{cccc}
-0.05 & 0.02 & 0.03 & 0 \\
0.1 & -0.115 & 0 & 0.015 \\
0.2 & 0 & -0.21 & 0.01 \\
0 & 0.25 & 0.125 & -0.375
\end{array}\right]
\end{aligned}
$$

The corresponding transition matrix for one-month sampling intervals $(\Delta T=1)$ and the corresponding approximates computed using Equation (2.3) arranged in matrix $\tilde{P}(\Delta T)$ are given by the following expressions:

$$
\exp (1 \cdot Q)=\left[\begin{array}{cccc}
0.965 & 0.018 & 0.017 & 0.000 \\
0.194 & 0.796 & 0.003 & 0.007 \\
0.148 & 0.002 & 0.843 & 0.007 \\
0.036 & 0.144 & 0.201 & 0.619
\end{array}\right], \tilde{P}(1)=\left[\begin{array}{cccc}
0.961 & 0.018 & 0.017 & 0.000 \\
0.194 & 0.793 & 0.003 & 0.007 \\
0.147 & 0.002 & 0.84 & 0.007 \\
0.036 & 0.144 & 0.201 & 0.618
\end{array}\right]
$$

Comparing these two matrices shows that $\tilde{P}(1)$ accounts for more than $99 \%$ of the probability of $\exp (1 \cdot Q)$ in each entry Table S2.1. With a longer sampling interval of 3 months, the proportion of accounted probability remains above $96 \%$. The proportion of probability covered by $\tilde{P}(\Delta T)$ remains high in models up to seven types, which is the highest number of types we have considered in this analysis. With 21 types, which is the number of pneumococcal serotypes in the motivating data set, the proportions of accounted probability we obtained by linear extrapolation of the values given in Table S2.1 which are $96 \%, 85 \%$ and $70 \%$ for sampling intervals of 1,2 and 3 months.

Table S2.1: Minimum proportion of the exact likelihood accounted for by the approximation across all element of matrices $\exp (\Delta T \cdot Q)$ and $\tilde{P}(\Delta T)$ for increasing number of types and length of sampling interval.

\begin{tabular}{c||ccc}
\hline Number of types & $\boldsymbol{\Delta} \boldsymbol{T}=\mathbf{1}$ & $\boldsymbol{\Delta} \boldsymbol{T}=\mathbf{2}$ & $\boldsymbol{\Delta} \boldsymbol{T}=\mathbf{3}$ \\
\hline 2 & $99.6 \%$ & $98.6 \%$ & $96.7 \%$ \\
3 & $99.5 \%$ & $98.0 \%$ & $95.5 \%$ \\
4 & $99.2 \%$ & $96.9 \%$ & $94.6 \%$ \\
5 & $99.1 \%$ & $95.8 \%$ & $93.1 \%$ \\
6 & $98.9 \%$ & $95.3 \%$ & $91.0 \%$ \\
7 & $98.7 \%$ & $94.8 \%$ & $89.2 \%$ \\
\hline
\end{tabular}

\section{The naive method based on midpoint imputation}

In the naive estimation method, the transition times are imputed midway between consecutive observation times. After imputation, maximum likelihood estimates of the model parameters are obtained using the following Poisson regression models with the baseline rates representing the intercepts, the presence/absence of other serotypes representing the independent variables, and the person-times 
representing the offsets:

$$
\begin{aligned}
& \log \left(\mathbb{E}\left(\left[\begin{array}{c}
Y_{1, a c q,-} \\
Y_{2, a c q,-} \\
\vdots \\
Y_{p, a c q,-} \\
\hline Y_{1, a c q,+} \\
Y_{2, a c q,+} \\
\vdots \\
Y_{p, a c q,+}
\end{array}\right]\right)\right)=\left[\begin{array}{cccc|c}
1 & 0 & \cdots & 0 & 0 \\
0 & 1 & \cdots & 0 & 0 \\
\vdots & & \ddots & \vdots & \vdots \\
0 & 0 & \cdots & 1 & 0 \\
\hline 1 & 0 & \cdots & 0 & 1 \\
0 & 1 & \cdots & 0 & 1 \\
\vdots & & \ddots & \vdots & \vdots \\
0 & 0 & \cdots & 1 & 1
\end{array}\right]\left[\begin{array}{c}
\lambda_{1} \\
\lambda_{2} \\
\vdots \\
\lambda_{p} \\
k
\end{array}\right]+\log \left(\left[\begin{array}{c}
T_{1, a c q,-} \\
T_{2, a c q,-} \\
\vdots \\
T_{p, a c q,-} \\
\hline T_{1, a c q,+} \\
T_{2, a c q,+} \\
\vdots \\
T_{p, a c q,+}
\end{array}\right]\right) \\
& \log \left(\mathbb{E}\left(\left[\begin{array}{c}
Y_{1, c l r,-} \\
Y_{2, c l r,-} \\
\vdots \\
Y_{p, c l r,-} \\
\hline Y_{1, c l r,+} \\
Y_{2, c l r,+} \\
\vdots \\
Y_{p, c l r,+}
\end{array}\right]\right)\right)=\left[\begin{array}{cccc|c}
1 & 0 & \cdots & 0 & 0 \\
0 & 1 & \cdots & 0 & 0 \\
\vdots & & \ddots & \vdots & \vdots \\
0 & 0 & \cdots & 1 & 0 \\
\hline 1 & 0 & \cdots & 0 & 1 \\
0 & 1 & \cdots & 0 & 1 \\
\vdots & & \ddots & \vdots & \vdots \\
0 & 0 & \cdots & 1 & 1
\end{array}\right]\left[\begin{array}{c}
\mu_{1} \\
\mu_{2} \\
\vdots \\
\mu_{p} \\
\hline h
\end{array}\right]+\log \left(\left[\begin{array}{c}
T_{1, c l r,-} \\
T_{2, c l r,-} \\
\vdots \\
T_{p, c l r,-} \\
\hline T_{1, c l r,+} \\
T_{2, c l r,+} \\
\vdots \\
T_{p, c l r,+}
\end{array}\right]\right)
\end{aligned}
$$

The terms in these equations are defined as follows:

- $Y_{j, a c q,-}$ : number of acquisition events of type $j$ in absence of other types;

- $Y_{j, a c q,+}$ : number of acquisition events of type $j$ in presence of other types;

- $T_{j, a c q,-}$ : person-time in absence of any types;

- $T_{j, a c q,+}$ : person-time in absence of type $j$ but in presence of other types;

- $Y_{j, c l r,-}:$ number of clearance events of type $j$ in absence of other types;

- $Y_{j, c l r,+}$ : number of clearance events of type $j$ in presence of other types;

- $T_{j, c l r,-}$ : person-time in presence of type $j$ but in absence of other types;

- $T_{j, c l r,+}$ : person-time in presence of type $j$ and in presence of other types.

\section{Derivation of the priors for the baseline rates}

The parameters of the gamma priors for baseline rates used in BALB were derived separately for each serotype and for acquisition and clearance rates. The shape and rate parameters of the gamma prior were chosen as $\alpha_{\text {baseline }}=0.00001$ and $\beta_{\text {baseline }}=\alpha_{\text {baseline }} / \nu_{\text {baseline. }}$. Here, $\nu_{\text {baseline }}$ is the crude mean, derived by dividing the number of acquisition or clearance events by the total at-risk persontime assuming transitions to be midway between observation times. This means that the prior mean is $\nu_{\text {baseline }}$ and the prior variance $\nu_{\text {baseline }}^{2} / \alpha_{\text {baseline. }}$.

\section{E. Supplementary tables of the simulation study}




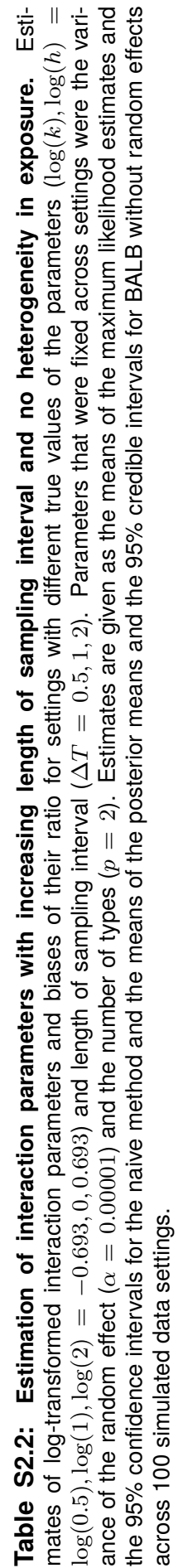

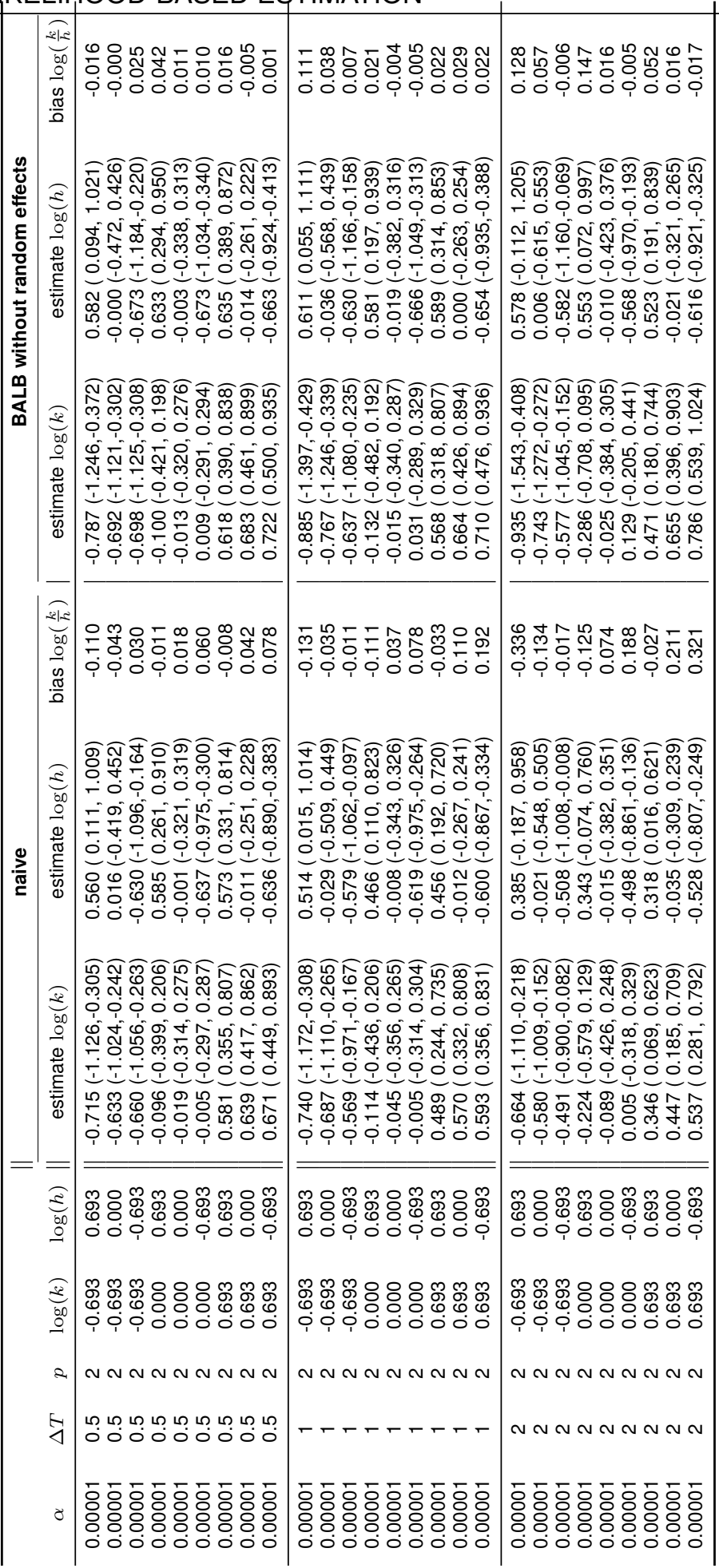




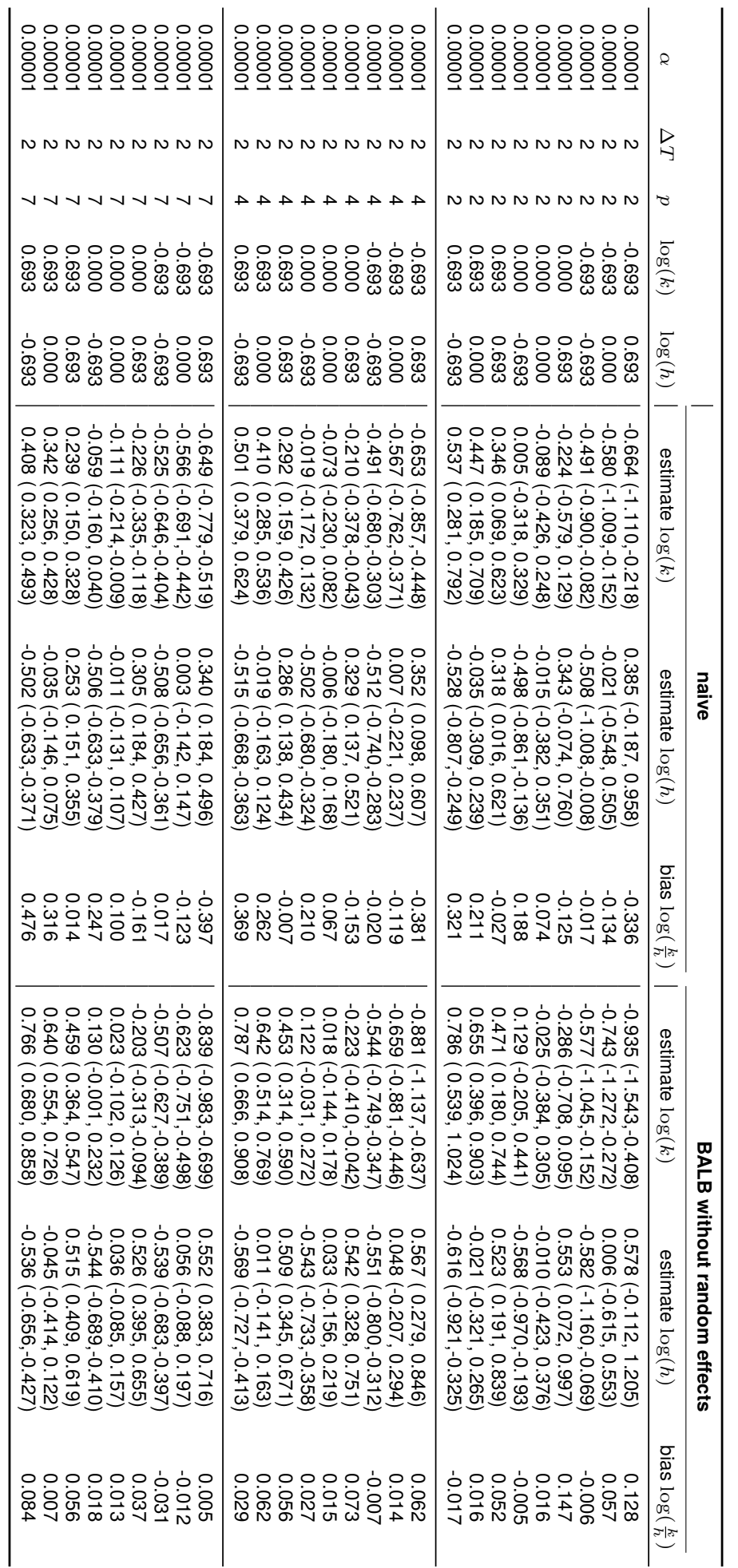

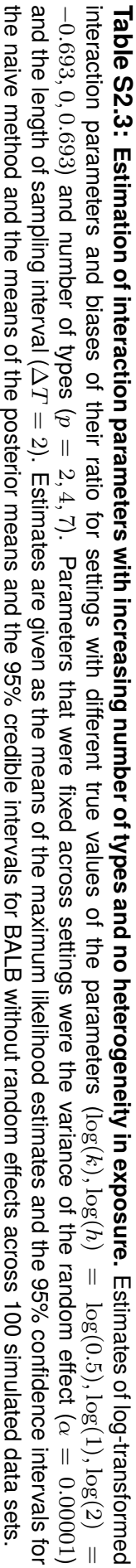




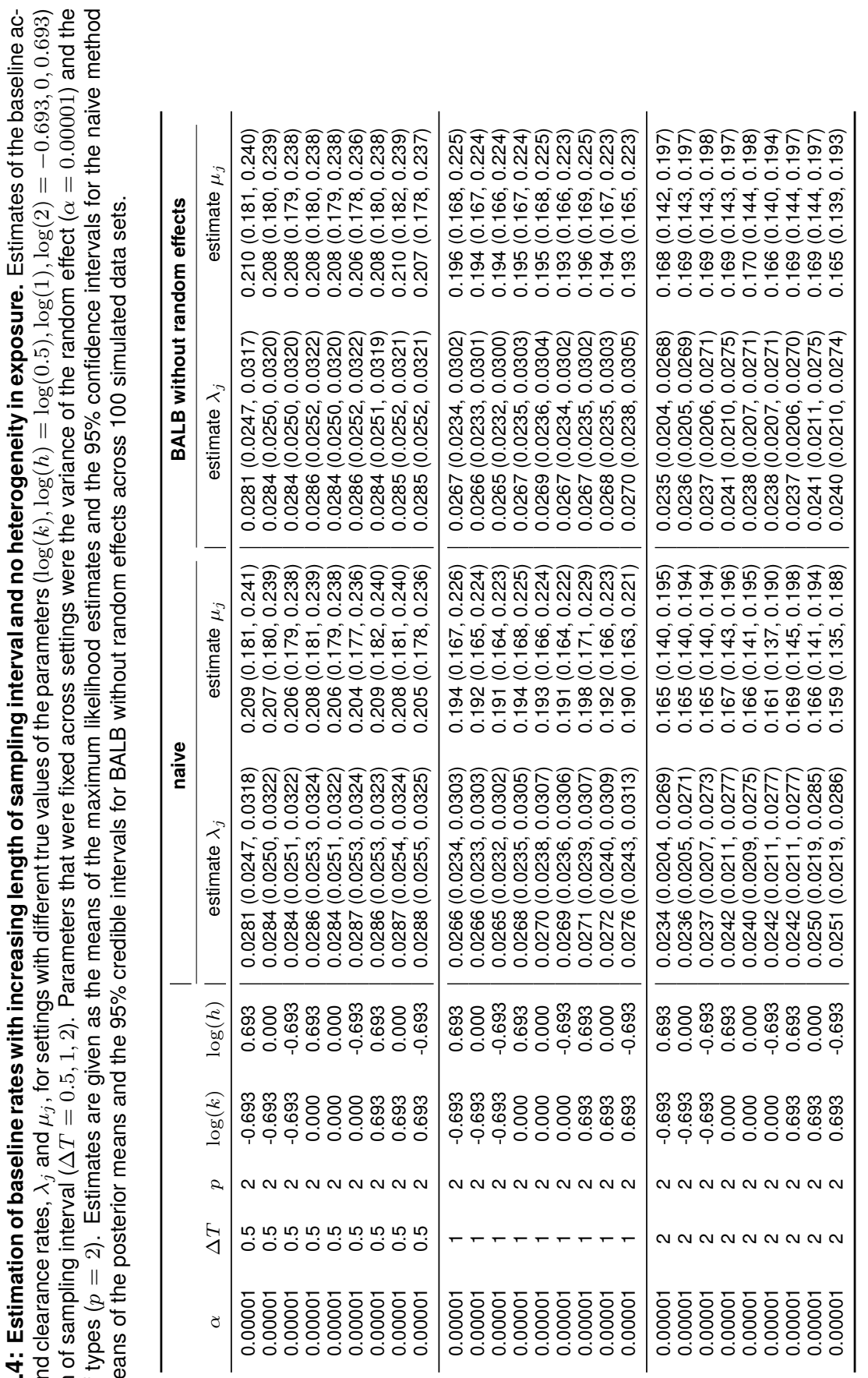




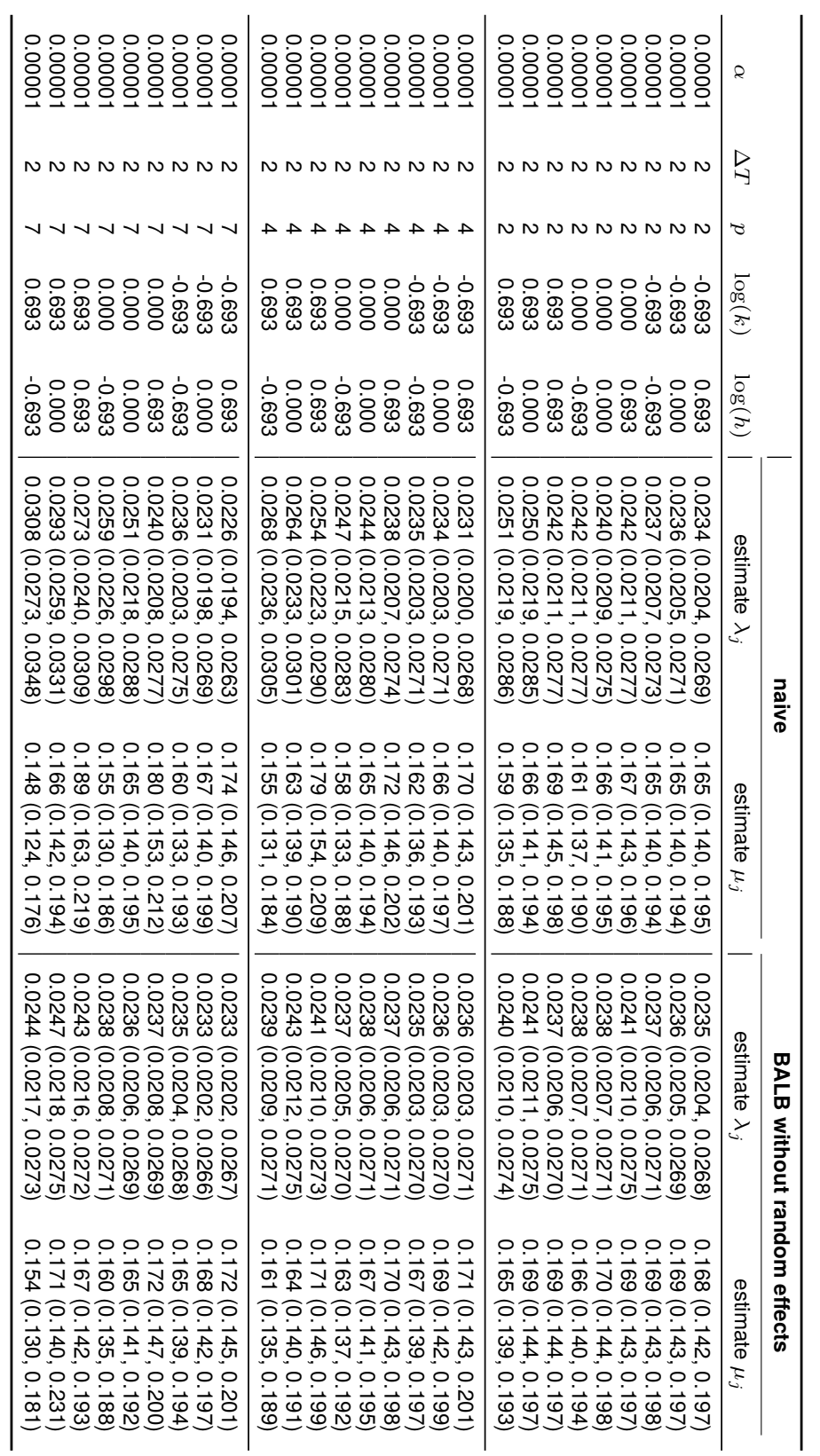




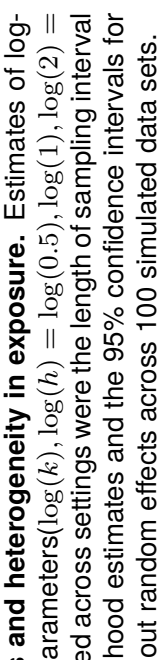

సٓ

专产市要

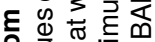

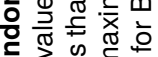

๙

$\div \stackrel{0}{0}$

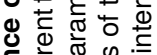

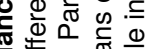

类

Oे

可 305

\%

过要家怘

훙워웡

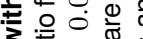

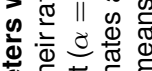

屯 焉

춘

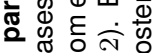

든 응 흔

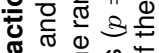

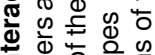

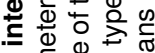

두

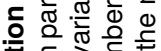

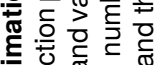

热宁市

山.

$\ddot{0}$

ฟิ है О न ญ

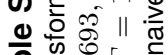

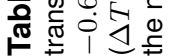

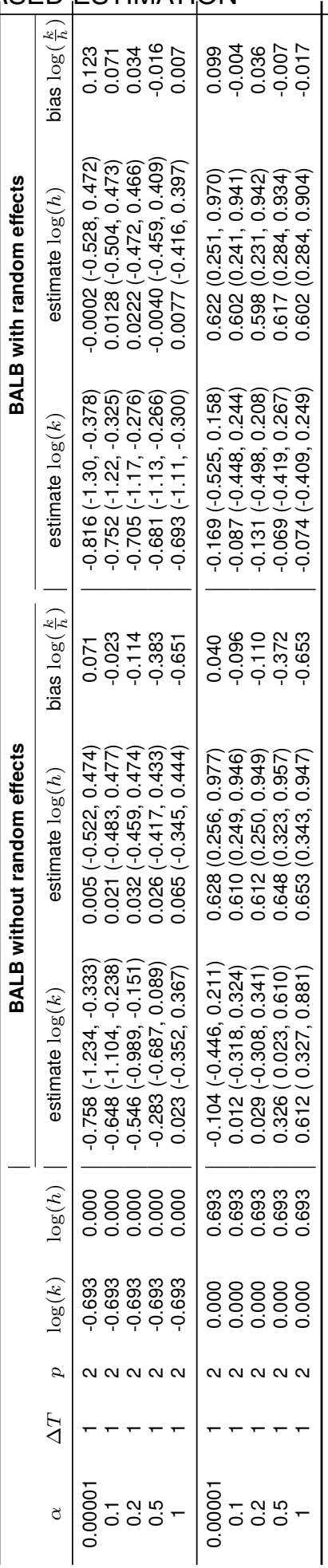




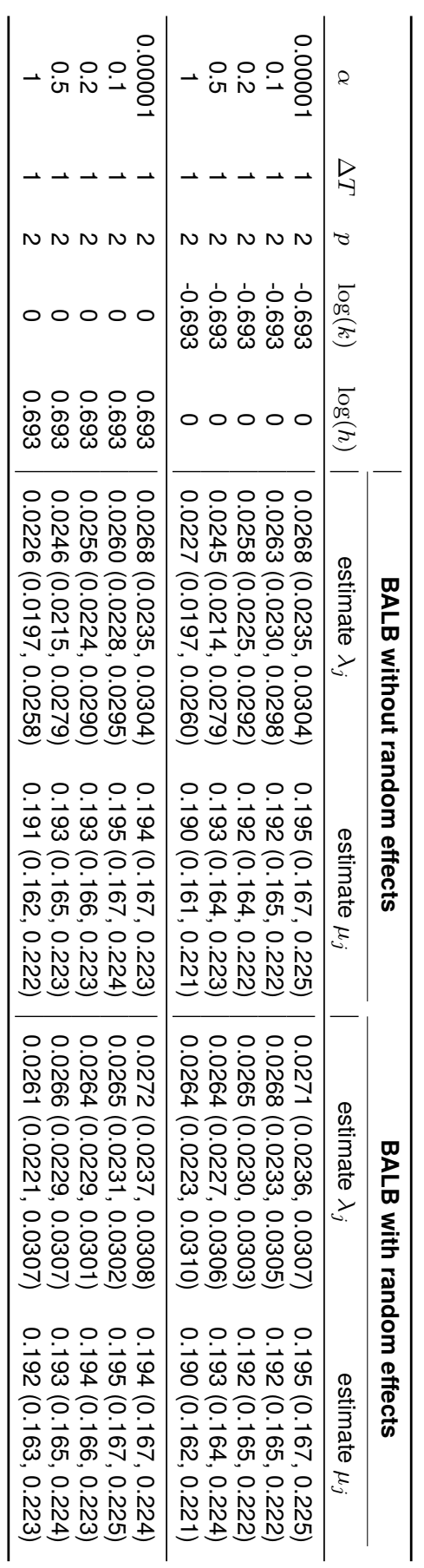

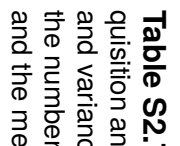

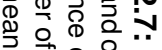

心芒檕罗

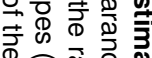

ণ

에응 응 흥

ه

욱 이웡

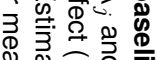

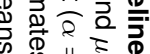

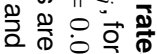

흔.

요욜

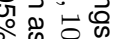

$\circ$ क

웅 穴证市

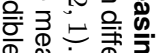

न

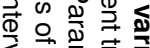

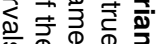

웅

일

四肴志

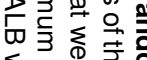

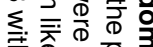

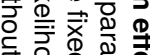

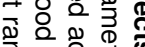

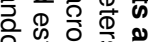

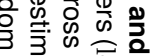

व

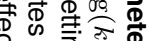

잉

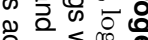

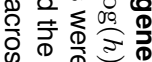
की 0 क 융휴

○ㅇำ

능. 응 옹

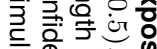

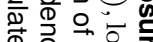

范

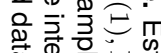

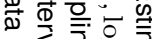

象 $\frac{\varrho}{\omega}$

क ज्ञ

윽 웃 응

完@! 丞

光.

\} \| 0 \stackrel { \mathbb { D } } { \mathbb { D } }

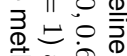

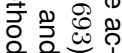




\section{F. Supplementary tables and figues of the pneumococcal data set}

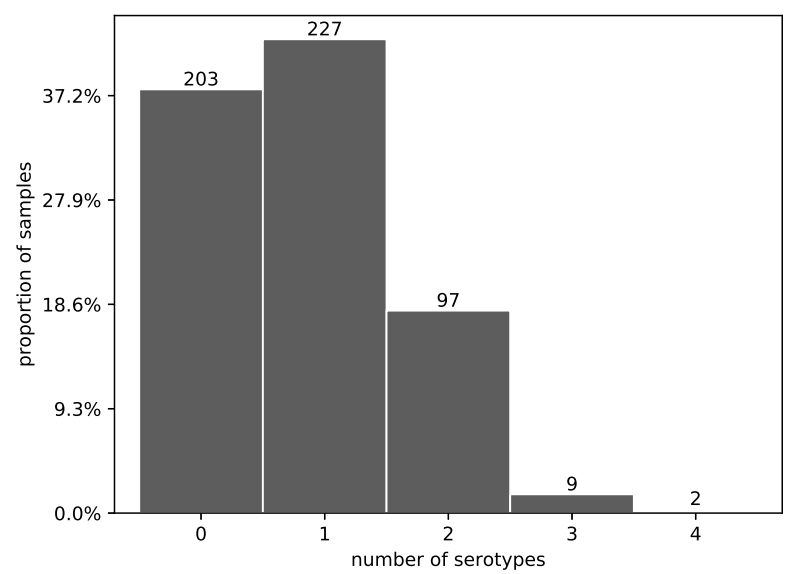

Figure S2.1: Proportion of all positive samples $(\mathrm{N}=538)$ by number of co-carried serotypes. 


\begin{tabular}{|c|c|c|c|c|}
\hline \& & 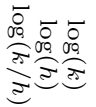 & 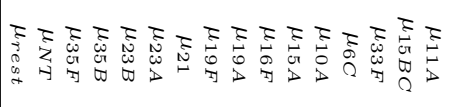 & 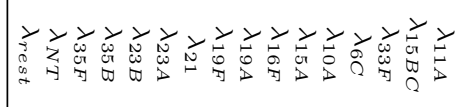 & \\
\hline & 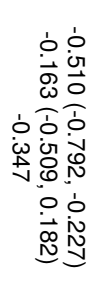 & 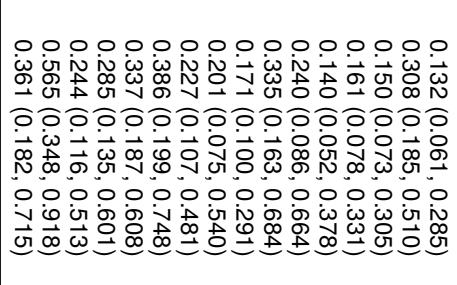 & 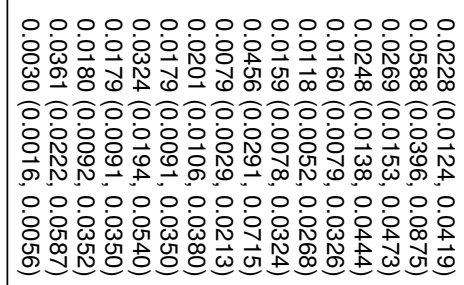 & 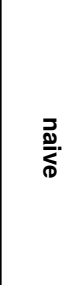 \\
\hline & 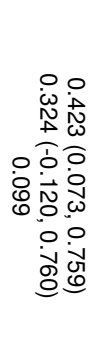 & 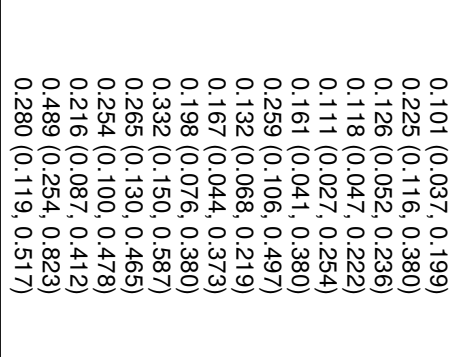 & 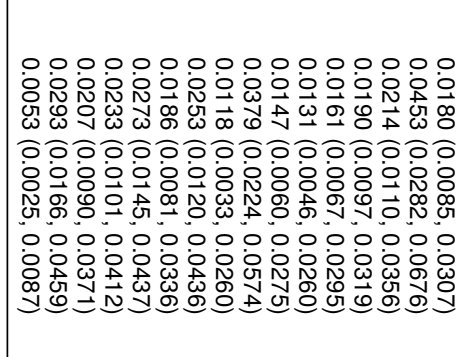 & 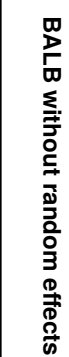 \\
\hline 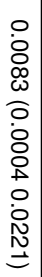 & 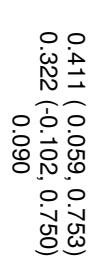 & 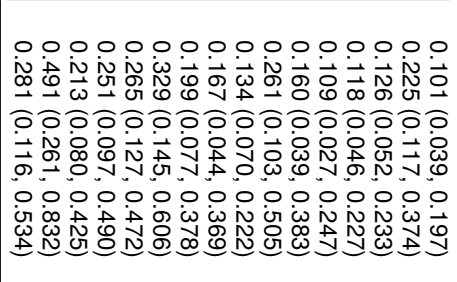 & 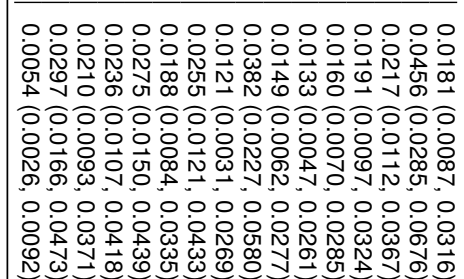 & $\begin{array}{l}\infty \\
\stackrel{0}{0} \\
\stackrel{0}{0}\end{array}$ \\
\hline $\begin{array}{l}0 \\
0 \\
0 \\
N \\
0 \\
0 \\
0 \\
0 \\
0 \\
0 \\
0 \\
\text { N } \\
\text { पn } \\
0\end{array}$ & 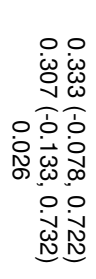 & 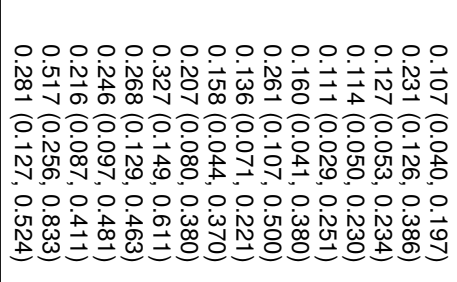 & 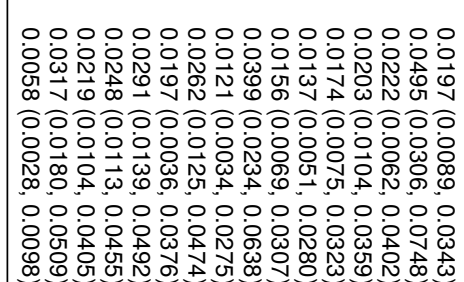 & 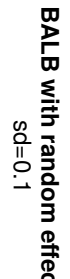 \\
\hline 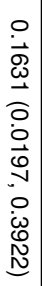 & 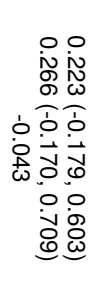 & 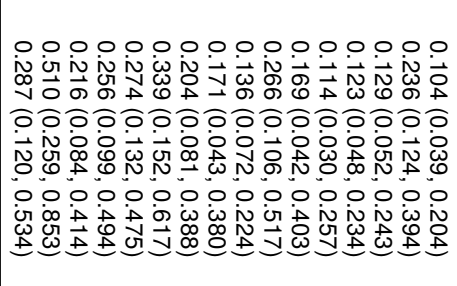 & 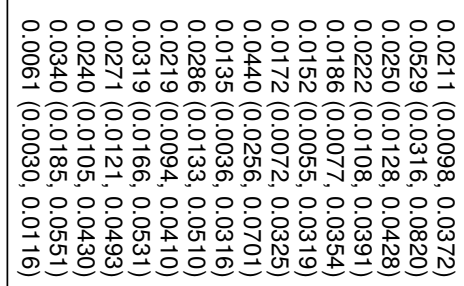 & $\stackrel{\text { ¿n }}{I 1}$ \\
\hline
\end{tabular}




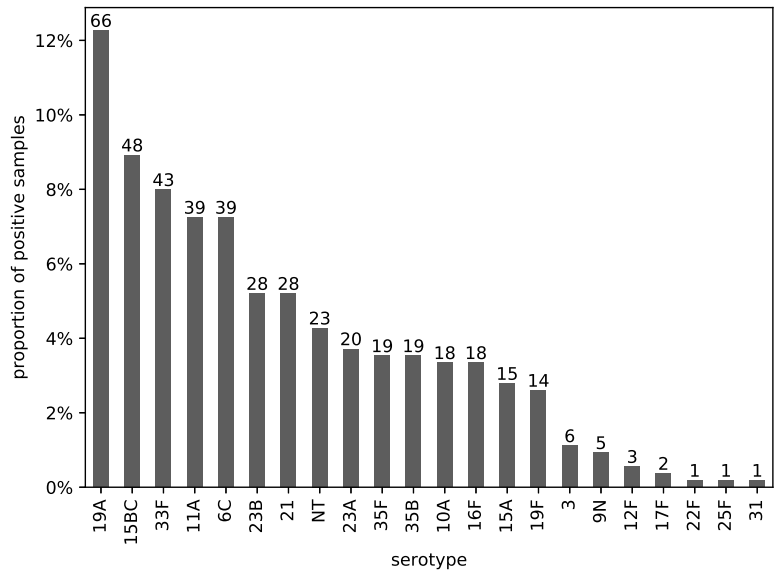

Figure S2.2: Proportion of samples positive for each serotype $(\mathrm{N}=538)$. Samples positive for $S$. pneumoniae for which the serotype could not be determined were assigned as non-typable (NT). 


\section{Chapter 3}

\section{Inferring pathogen type interactions using cross- sectional prevalence data: opportunities and pitfalls for predicting type replacement}

This chapter has been published as: I. Man, J. Wallinga, and J. A. Bogaards. "Inferring pathogen type interactions using cross-sectional prevalence data: opportunities and pitfalls for predicting type replacement”. Epidemiology, 29.5 (2018), 666-674. 


\section{Abstract}

\section{Background}

Many multi-valent vaccines target only a subset of types of a pathogen. If vaccine and non-vaccine types compete, vaccination may lead to type replacement. The plausibility of type replacement has been assessed using the odds ratio $(O R)$ of co-infections in cross-sectional prevalence data, with $O R>1$ being interpreted as low risk of type replacement. The usefulness of the $O R$ as a predictor for type replacement is debated, as it lacks a theoretical justification, and there is no framework explaining under which assumptions the $O R$ predicts type replacement.

\section{Methods}

We investigate the values that the $O R$ can take based on deterministic $S$ usceptibleInfected-S usceptible and $S$ usceptible-Infected-Recovered-Susceptible multi-type transmission models. We consider different mechanisms of type interactions and explore parameter values ranging from synergistic to competitive interactions.

\section{Results}

We find that $O R>1$ might mask competition because of confounding due to unobserved common risk factors and cross-immunity, as indicated by earlier studies. We prove mathematically that unobserved common risk factors lead to an elevation of the $O R$ and present an intuitive explanation of why cross-immunity increases the $O R$. We find that $O R<1$ is predictive for type replacement in the absence of immunity. With immunity, $O R<1$ remains predictive under biologically reasonable assumptions of unidirectional interactions during infection and an absence of immunity-induced synergism.

\section{Conclusions}

Using the $O R$ in cross-sectional data to predict type replacement is justified but is only unambiguous under strict assumptions. An accurate prediction of type replacement requires pathogen-specific knowledge on common risk factors and cross-immunity. 


\section{Introduction}

Studying and predicting the effects of vaccination against pathogens with many types can be challenging if the types interact with each other [1]. With a vaccine that immunises against only a subset of pathogen types, vaccination may indirectly affect the types that are not targeted. Vaccination against the vaccine types may increase or decrease the prevalence of the non-vaccine types, depending on whether the interactions between the vaccine and non-vaccine types are competitive or synergistic. If the interactions are synergistic, vaccination may decrease the prevalence of the non-vaccine types since it also takes away the synergistic effects that the non-vaccine types receive from the vaccine types. If the interactions are competitive, vaccination may increase the prevalence of the non-vaccine types so that the non-vaccine types replace the vaccine types [2-4]. Such replacements have been observed after the introduction of vaccination against pathogens like Haemophilus influenzae and Streptococcus pneumoniae [5-7]. For human papillomavirus (HPV), it is still unclear if different genotypes interact and whether vaccination will lead to type replacement [8, 9].

To assess the risk of type replacement before the introduction of vaccination, investigators have searched for evidence of competition between the vaccine and non-vaccine types in epidemiologic studies. In particular, cross-sectional prevalence studies may provide information on patterns of co-occurrence of pathogen types. Co-occurrence can be defined as co-infection by different virus types (e.g. for HPV) or as co-carriage of different bacterial types (e.g. for S. pneumoniae). Once the meaning of co-occurrence is defined, type interactions can be quantified by the observed number of co-occurrences. Deviations of this quantity from the expected number of co-occurrences under independence can be interpreted as evidence for interactions. A common measure of association that expresses the extent of such deviation is the odds ratio $(O R)$ of co-occurrence, with positive (negative) associations being considered indicative of synergistic (competitive) interactions.

Although this qualitative interpretation of associations has an intuitive appeal, it may not be consistent with the underlying mechanisms of interactions, leading to an incorrect assessment of the risk of type replacement. While some competitive mechanisms induce negative associations, others, such as cross-immunity, have been shown to induce positive associations [10, 11]. Even if the underlying mechanism of interactions agrees with this intuitive interpretation, type interactions may be confounded by unobserved risk factors or routes of transmission that are shared by multiple types, leading to a bias toward positive associations [8, 11, 12].

For HPV, various cross-sectional prevalence studies from the pre-vaccination era found co-infections to occur more often than expected, expressing positive associations, but few noteworthy differences between type-specific associations 
are reported [13-15]. Such co-infection patterns are usually explained in terms of unobserved common risk factors, with low risk of type replacement according to the intuitive interpretation. However, it is not clear to what extent competitive interactions may be masked by common risk factors, and therefore whether type replacement following HPV vaccination is plausible. Moreover, it remains to be demonstrated whether unobserved common risk factors have the same effect on each type-specific association and can be corrected for.

Although the validity of using the $O R$ of co-occurrence for inferring type interactions has been studied before [10, 11, 16], its methodologic basis is not yet well established. In this chapter, we 1) derive the $O R$ as an estimator of interactions in acquisition and clearance; 2) present a proof for positive bias due to unobserved common risk factors; 3) provide a novel explanation of how cross-immunity induces positive associations. Ultimately, our goal is to assess the usefulness of co-occurrence patterns of pathogen types in cross-sectional prevalence data for predicting type replacement.

\section{The $O R$ as an estimator of the interaction parameters}

\section{A $S$ usceptible-Infected- $S$ usceptible model with two pathogen types}

We first consider a $S$ usceptible-Infected-Susceptible $(S I S)$ model without two pathogen types in a closed population (Figure 3.1A) [3, 17]. In this population, individuals are susceptible or infected with respect to each of the two types so that there are $2^{2}=4$ different infection states. We encode each of the infection states by a notation in which the $i$-th letter indicates the status with respect to the $i$-th type: $S$ for susceptible and $I$ for infected. In formulae, each of $\{S S, I S, S I, I I\}$ denotes the proportion of individuals in the corresponding state in the population. Together, they give the joint distribution of the two types.

In this model, individuals without any infections $(S S)$ become infected by type 1 at rate $\lambda_{1}=c \beta_{1}(I S+I I)$ and by type 2 at rate $\lambda_{2}=c \beta_{2}(S I+I I)$, where $c$ is the contact rate and $\beta_{i}$ the probability of acquiring type $i$ given established contact with an infected individual.

Types interact through two mechanisms: acquisition and clearance. Due to interactions in acquisition, individuals already infected by one type acquire an infection of the other type at an adjusted rate that is $k$ times $\lambda_{1}$ or $\lambda_{2}$. The interaction parameter $k$ is essentially the rate ratio of acquiring infections of one type among individuals that are already infected by the other type (exposed) compared with the acquisition among susceptibles (unexposed). Similarly, due to interactions in clearance, individuals that are infected by both types clear infections at an adjusted rate that is $h$ times the clearance rate of individuals that are infected by only one type, $\mu_{1}$ or $\mu_{2}$. The interaction parameter $h$ is therefore the rate ratio of 

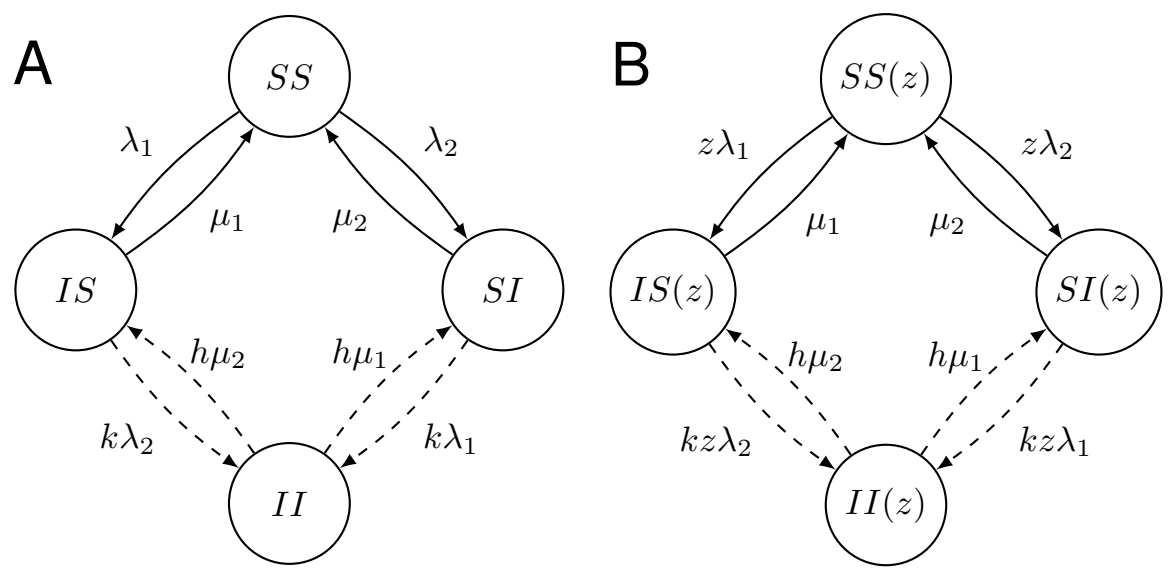

Figure 3.1: The structure of two SIS models for two pathogen types with interactions in acquisition and clearance indicated by dashed arrows. We encode each of the infection states by a notation in which the $i$-th letter indicates the status with respect to the $i$-th type: $S$ for susceptible and $I$ for infected. The susceptibility is assumed to be homogeneous in (A) and heterogeneous in (B). (B) depicts only the subpopulation with susceptibility level $z$.

clearing infections of one type among individuals infected by the other type (exposed) compared with the clearance among individuals not infected by the other type (unexposed). Interactions in acquisition (clearance) can be either independent, synergistic, or competitive by choosing $k$ to be $=$, , or $<1\left(\frac{1}{h}\right.$ to be $=$, $>$, or $<1$ ) as given by Table 3.1.

The following system of differential equations describes how $\{S S, I S, S I, I I\}$ changes over time:

$$
\left\{\begin{array}{l}
\frac{d S S}{d t}=-\left(\lambda_{1}+\lambda_{2}\right) S S+\mu_{1} I S+\mu_{2} S I \\
\frac{d I S}{d t}=\lambda_{1} S S-\left(\mu_{1}+k \lambda_{2}\right) I S+h \mu_{2} I I \\
\frac{d S I}{d t}=\lambda_{2} S S-\left(\mu_{2}+k \lambda_{1}\right) S I+h \mu_{1} I I \\
\frac{d I I}{d t}=k \lambda_{2} I S+k \lambda_{1} S I-h\left(\mu_{1}+\mu_{2}\right) I I
\end{array}\right.
$$

Table 3.1: Parameter values for interaction parameters $k$ and $h$ corresponding synergistic, independent, and competitive interactions.

\begin{tabular}{c||ccc}
\hline Operating mechanism & Synergy & Independence & Competition \\
\hline Acquisition & $k>1$ & $k=1$ & $k<1$ \\
Clearance & $\frac{1}{h}>1$ & $\frac{1}{h}=1$ & $\frac{1}{h}<1$ \\
\hline
\end{tabular}




\section{The stationary distribution}

$\{S S, I S, S I, I I\}$, as governed by Equation (3.1) always stabilises at an equilibrium as time progresses. This occurs for any given set of model parameters. We assume that the model parameters are chosen such that both types are present (coexisting) at the equilibrium.

Equilibria in the deterministic setting are closely related to stationary distributions in Markov processes, since both are stable in time. In this paper, we consider the setting in which a cross-sectional dataset consists of individuals sampled from a stationary distribution that coincides with the equilibrium of the system described by Equation (3.1)

As we are interested in the cross-sectional setting, we focus on the equilibrium and neglect transient dynamics of $\{S S, I S, S I, I I\}$. By solving the linear system that governs the equilibrium (see Appendix A), we obtain the following simple expression in terms of $\left\{\lambda_{1}, \lambda_{2}, \mu_{1}, \mu_{2}, k, h\right\}$ :

$$
\left\{\begin{array}{l}
S S=\left(h \mu_{1} \mu_{2}\right) / C \\
I S=\left(h \mu_{2} \lambda_{1}\right) / C \\
S I=\left(h \mu_{1} \lambda_{2}\right) / C \\
I I=\left(k \lambda_{1} \lambda_{2}\right) / C,
\end{array}\right.
$$

where $C=h \mu_{1} \mu_{2}+h \mu_{2} \lambda_{1}+h \mu_{1} \lambda_{2}+k \lambda_{1} \lambda_{2}$ is the normalizing constant. Note that the derivation of Equation (3.2) does not depend on how $\lambda_{i}$ is defined in terms of the contact rate and transmission probabilities so that Equation (3.2) holds as long as the model has the structure depicted in Figure 3.1. A.

\section{Result I: The $O R$ is an exact estimator of the composite of the inter- action parameters, $\frac{k}{h}$.}

The $O R$ is defined as the ratio of the odds of one type in presence of the other type, relative to the odds of this type in absence of the other type:

$$
O R=\left(\frac{I I}{S I}\right) /\left(\frac{I S}{S S}\right) \text {. }
$$

To compute the $O R$, Equation (3.2) is substituted in Equation (3.3) This substitution yields $O R=\frac{k}{h}$, a function of the composite of the interaction parameters. If only one mechanism of interactions is operating, that is, $h=1$ or $k=1$, the $O R$ reduces to $k$ or the reciprocal of $h$ as shown in Figure 3.2A, respectively. An alternative proof of $O R=\frac{k}{h}$ based on reversibility can be found in Appendix A. 

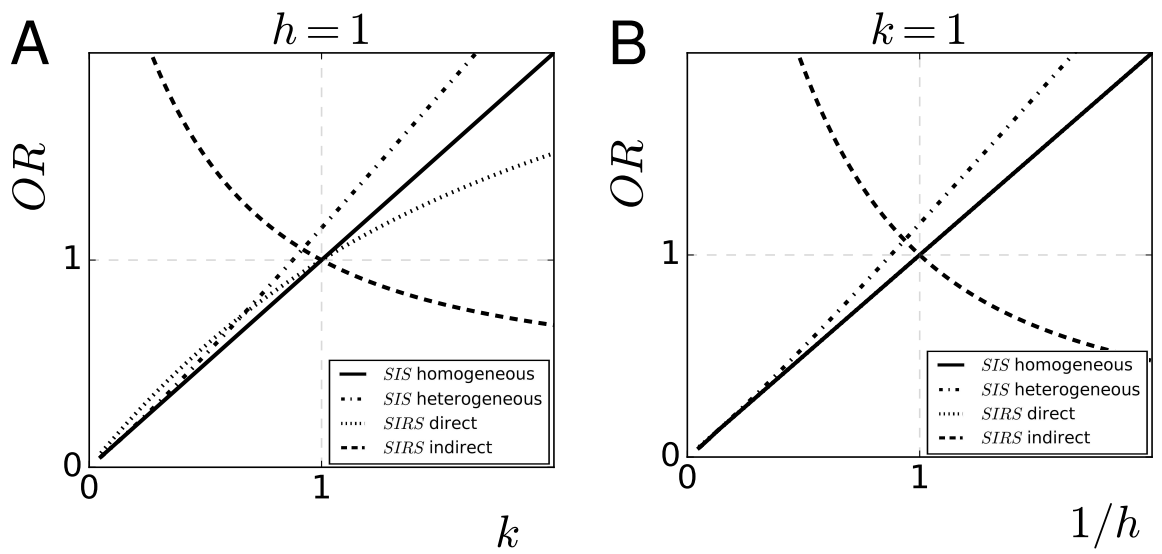

Figure 3.2: The crude $O R$ as an estimator of the interaction parameter for acquisition $(k)$ in $(\mathrm{A})$ and the reciprocal of the interaction parameter for clearance $\left(\frac{1}{h}\right)$ in (B). Homogeneous SIS model (solid): unbiased estimation. Heterogeneous SIS model (dasheddotted): overestimation. SIRS model with direct interactions (dotted): overestimation of $k<1$ and underestimation of $k>1$, unbiased estimation of $h$ (overlapped by the solid line). SIRS model with indirect interactions (dashed): reversion bias.

\section{The $O R$ as a predictor for type replacement}

\section{The outcome of vaccination}

We investigate whether the $O R$ correctly predicts the outcome of vaccination. We introduce the following indicator function to denote the outcome for the prevalence of type 1 (non-vaccine type) when vaccinating against type 2 (vaccine type):

$$
\Phi=\left\{\begin{array}{l}
+, \text { if the prevalence of type } 1 \text { decreases } \\
\circ, \text { if the prevalence of type } 1 \text { stays unchanged } \\
-, \text { if the prevalence of type } 1 \text { increases (type replacement). }
\end{array}\right.
$$

Hence, $\Phi=+$ denotes a beneficial and $\Phi=-$ an unfavorable impact on the nonvaccine type due to vaccination against the vaccine type. We simulate vaccination by reducing the probability of acquiring type 2 throughout the entire population and investigate whether $O R>,=,<1$ correctly predicts $\Phi=+, \circ,-$, respectively.

In the simulations, different parameter values of $k$ and $h$, ranging from competitive to synergistic interactions, lead to different outcomes of vaccination (Table 3.2. If one mechanism of interactions is independent $\left(k=1\right.$ or $\left.\frac{1}{h}=1\right), \Phi$ is determined by the parameter value of the other mechanism of interactions with respect to 1 . If both mechanisms of interactions are operating, we found the out- 
Table 3.2: Parameter values for the interaction parameters $k$ and $h$ corresponding $\Phi=$ $+, \circ,-$.

\begin{tabular}{c||ccc}
\hline Operating mechanisms & $\mathbf{\Phi}=+$ & $\mathbf{\Phi}=0$ & $\mathbf{\Phi}=-$ \\
\hline Independent in clearance & $k>1$ & $k=1$ & $k<1$ \\
Independent in acquisition & $\frac{1}{h}>1$ & $\frac{1}{h}=1$ & $\frac{1}{h}<1$ \\
Both operating & $\frac{k}{h}>1$ & $\frac{k}{h}=1$ & $\frac{k}{h}<1$ \\
\hline
\end{tabular}

come of vaccination to be determined by the value of $\frac{k}{h}$ with respect to 1 . As such, with both mechanisms operating in opposite directions, one being competitive and the other synergistic, the outcome of vaccination is determined by the strongest of the two (i.e. the parameter that deviates the most from 1).

\section{Result II: The $O R$ is a predictor for type replacement.}

As $O R=\frac{k}{h}$ and the value of $\frac{k}{h}$ with respect to 1 determines the value of $\Phi$, the $O R$ is a predictor for the outcome of vaccination; $O R>,=,<1$ predicts $\Phi=+, \circ,-$, respectively. This correspondence justifies the intuitive interpretation of the $O R$.

If the assumptions of the model are violated, the $O R$ may no longer be a predictor for the outcome of vaccination. In case the interactions are not symmetrical among types, the $O R$ becomes a weighted average of the type-specific interaction parameters. If the vaccine type is competitive towards the non-vaccine type, but the non-vaccine type is synergistic towards the vaccine type, $O R>1$ may hold even though type replacement does occur. Nevertheless, in less extreme cases of asymmetry, e.g. if the vaccine and non-vaccine type are both competitive or both synergistic towards each other but with different strength, the $O R$ still correctly predicts the outcome of vaccination.

\section{Positive bias due to unobserved common risk fac- tors}

Individuals may differ in risk of infections because of differences in genetic disposition or behaviour. Some risk factors are common for all pathogen types. If a common risk factor is not observed nor adjusted for, it may confound the previous result of the $O R$ being an estimator of $\frac{k}{h}$ and a predictor for type replacement. Using an example with unobserved heterogeneity in susceptibility, we illustrate the confounding effect due to unobserved common risk factors and explain why the bias is towards positive associations. 


\section{Heterogeneity in susceptibility}

We consider a heterogeneous $S$ usceptible-Inected-Susceptible model in which each individual is assigned an unobserved susceptibility level $z$, which influences his/her susceptibility for both types. The variation of $z$ in the population is captured by the density function $f(z)$.

As this model comprises an extra dimension, $z$, the proportions of different infection states at the equilibrium become functions of $z:\{S S(z), I S(z), S I(z), I I(z)\}$. To be consistent with the notations of the previous model, we let $\{S S, I S, S I, I I\}$ be the proportions of all individuals in the corresponding infection states regardless of the value of $z$, i.e. for $A=S S, I S, S I, I I$ :

$$
A=\int_{0}^{\infty} A(z) d z .
$$

We assume homogeneous mixing between individuals with different susceptibility levels. To model transmission, we define the global force of infection as $\lambda_{1}=c \beta_{1}(I S+I I)$ and $\lambda_{2}=c \beta_{2}(S I+I I)$. We then define the individual-specific force of infection to be the product of the individual-specific multiplier, $z$, and the global force of infection: $z \lambda_{1}$ and $z \lambda_{2}$. In Figure 3.1 $\mathrm{B}$, the infection dynamics of the population with susceptibility level $z$ is shown. The corresponding system of differential equations can be found in Appendix B.

\section{The crude and the adjusted $O R$}

If the susceptibility level is not observed, the crude $O R$ is computed without distinguishing between individuals with different susceptibility levels:

$$
O R=\left(\frac{I I}{S I}\right) /\left(\frac{I S}{S S}\right)
$$

For the hypothetical situation in which we could observe the susceptibility level, we define the adjusted $O R$ to be the $O R$ evaluated at each $z$ :

$$
O R(z)=\left(\frac{I I(z)}{S I(z)}\right) /\left(\frac{I S(z)}{S S(z)}\right) .
$$

For each fixed susceptible level, $z$, the corresponding system of differential equations follows the same structure as the one of the homogeneous $S$ usceptibleInected-S usceptible model, where $\lambda_{i}$ in Equation (3.1) is replaced by $z \lambda_{i}$. Hence, for each $z$, the adjusted $O R$ remains an estimator of $\frac{k}{h}$. 


\section{Result III: The crude $O R$ overestimates the composite of interaction parameters, $\frac{k}{h}$, and is not a sensitive predictor for type replacement.}

The crude $O R$ has a bias towards positive associations: $O R>\frac{k}{h}$. The proof of $O R>\frac{k}{h}$ in the case of independent interactions $\left(k=\frac{1}{h}=1\right)$ can be found in Appendix B. The proof invokes Chebyshev's integral inequality [18], which formalises a sufficient condition for a positive bias. In this example of heterogeneity in susceptibility, this condition requires the marginal probability of being infected to be either increasing or decreasing with $z$ for both types. This condition is satisfied since the higher the susceptibility level, the higher the probability of being infected.

Since the crude $O R$ overestimates $\frac{k}{h}, O R>1$ does not necessarily correspond to $\Phi=+$. However, $O R<1$ still corresponds to $\Phi=-$. In other words, $O R>1$ cannot rule out the possibility of type replacement, but $O R<1$ can predict it.

Using $O R=1$ as a threshold to distinguish between the presence and absence of type replacement leads to an incorrect prediction. However, there is no appropriate threshold value for the $O R$ that can produce a correct prediction, since the value of the $O R$ at $\Phi=\circ$ also depends on the chosen model parameters such as $\beta_{i}$ and $\mu_{i}$. Figure 3.3 shows the varying value of the $O R$ under independence for different combinations of $\beta_{1}, \beta_{2}$ (on the left) and $\mu_{1}, \mu_{2}$ (on the right). This dependency on type-specific parameters suggests that different typeto-type combinations may require different adjustments for the same unobserved common risk factors.

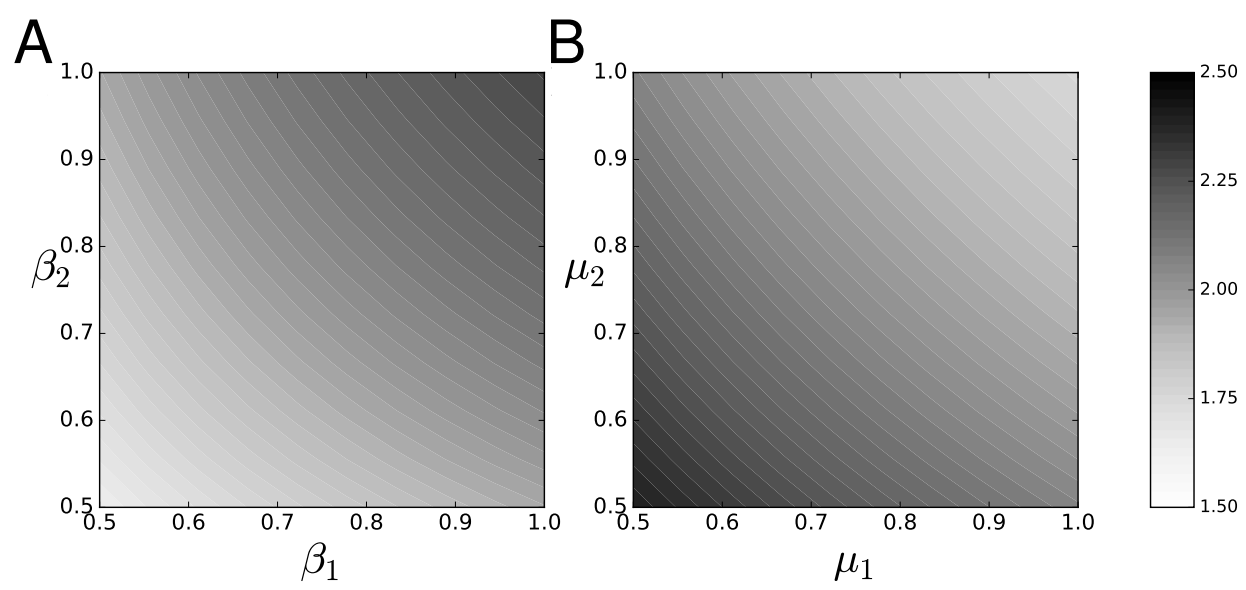

Figure 3.3: The crude $O R$ under independence in the heterogeneous $S I S$ model depends on type-specific parameters. A: it varies as $\beta_{1}$ and $\beta_{2}$ vary, while $\mu_{1}=\mu_{2}=1$. B: it varies as $\mu_{1}$ and $\mu_{2}$ vary, while $\beta_{1}=\beta_{2}=\frac{4}{7}$. For both $\mathrm{A}$ and $\mathrm{B}, c=3$ and $f(z)$ is a discrete distribution with $20 \%-80 \%$ mass at $z=0.2$ and $z=1.8$, respectively.

Unobserved heterogeneity in other variables, that are either negatively or pos- 
itively correlated with being infected with respect to both types, also leads to a positive bias of the $O R$ for $\frac{k}{h}$. For instance, heterogeneity in contact rate or clearance rate also leads to an overestimation of $\frac{k}{h}$, since the sufficient condition for positive bias we propose is satisfied. With assortative mixing according to such a common risk factor, we expect an even stronger positive bias [19].

\section{Different forms of bias due to type-specific or cross-immunity}

The results obtained under the $S$ usceptible-Inected-Susceptible model may not hold if natural infections trigger immune responses that protect the host against future infections. In general, immunity can be type-specific or cross-protective. For HPV, the strength of naturally acquired immunity is still a topic of discussion as is the possibility of cross-protection to related genotypes [20]. For instance, for S. pneumoniae, naturally acquired immunity is thought to build up with age and likely plays a minor role in transmission dynamics among toddlers but might mask competition among adults [21].

In this section, we analyse how type-specific immunity and cross-immunity affect the estimation of interaction parameters and the prediction of type replacement. We study the two $S$-usceptible-Infected- $R$-ecovered-Susceptible (SIRS) models depicted by Figure 3.4A and Figure 3.4B. The corresponding systems of differential equations can be found in Appendix C. In both models, we incorporate type-specific immunity by expanding the infection dynamics to $S I R S$ with regard to each type, where state $R$ (for Recovered) represents the immune state. The number of infection states now becomes $3^{2}=9$. Individuals enter state $R$ after clearance of infection and exit due to waning of immunity at rate $\gamma_{i}$ for type $i$. After losing immunity, individuals return to state $S$. In the $S I R S$ model given by Figure 3.4A, we keep the "SI"-part of the infection dynamics the same as in the previous $S I S$ model, including how types interact in acquisition and clearance. Hence, current infections of one type affect susceptibility for and clearance of the other type. In the SIRS model given by Figure 3.4B, we let past infections of one type affect susceptibility for and clearance of the other type. Such a mechanism of interactions is sometimes called indirect, since current infections of one type indirectly, through recovery, affect the other type. Indirect interactions, if competitive, correspond to cross-immunity, in which case being immune for one type offers protection to the other type. We consider direct and indirect interactions in separate models, since they lead to qualitatively different kinds of bias.

After incorporating the immune state, the definition of the $O R$ as given under 

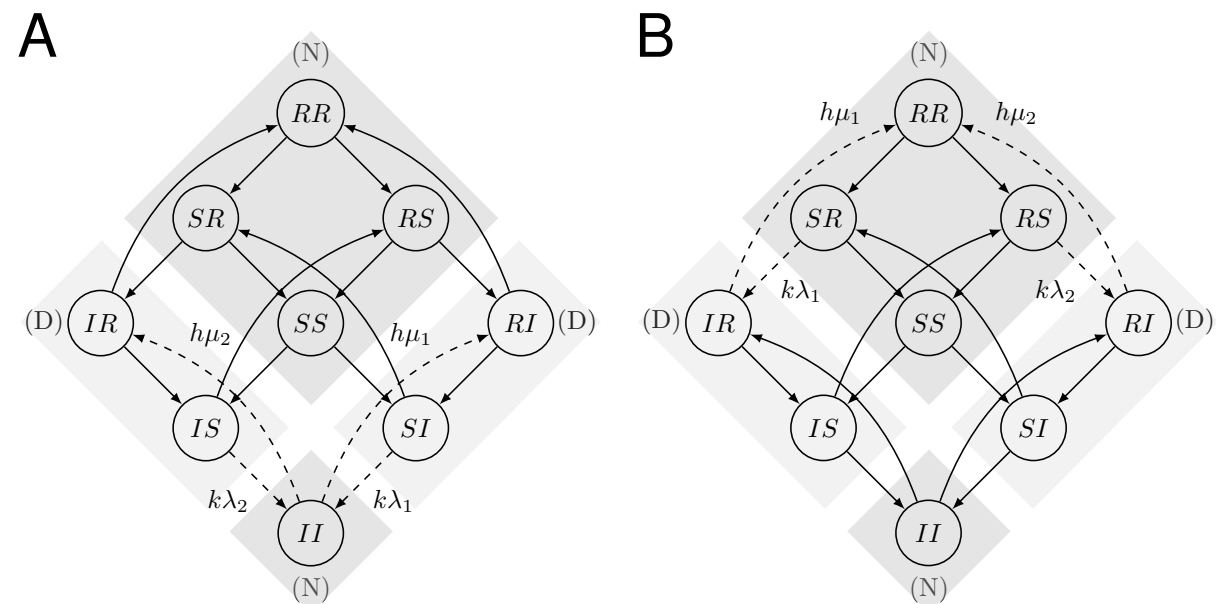

Figure 3.4: The structure of two SIRS models for two pathogen types with direct interactions in $(A)$ and indirect interactions in $(B)$ indicated by the dotted arrows. The transition rates not affected by type interactions are omitted. The dark grey areas indicate the terms in the numerator $(\mathrm{N})$ of the $O R$, whereas the light grey areas indicate the terms in the denominator (D) of the $O R$, where $O R=\frac{I I \cdot(S S+S R+R S+R R)}{(S I+R I) \cdot(I S+I R)}=\frac{(N)}{(D)}$.

result I becomes:

$$
O R=\left(\frac{I I}{S I+R I}\right) /\left(\frac{I S+I R}{S S+S R+R S+R R}\right) .
$$

This definition matches the empirical setting where one cannot distinguish between susceptible and immune individuals.

\section{Result IV: With type-specific immunity, the $O R$ is a biased estimator of the composite of the interaction parameters, $\frac{k}{h}$.}

In the SIRS model with direct interactions (Figure 3.4A), the $O R$ remains an unbiased estimator of $\frac{1}{h}$, but not of $k$ unless $k=1$ (see Figure 3.2A and Figure 3.2 3 ). $O R=1$ still constitutes a valid boundary between synergy and competition, however, the $O R$ overestimates $k$ if $k<1$ and underestimates $k$ if $k>1$. Jointly, the $O R$ becomes biased for $\frac{k}{h}$ (see Appendix $\mathrm{C}$ for the proof).

\section{Result V: With cross-immunity, the correspondence between the $O R$ and the composite of the interaction parameters, $\frac{k}{h}$, is reversed.}

In the SIRS model with indirect interactions (Figure 3.4 3 ), we consider crossimmunity as a composite of competition in both acquisition and clearance: past 
infections of one type hinder the acquisition and accelerate the clearance of the other type. We found that parameter values corresponding to cross-immunity ( $k<$ 1 and $\left.\frac{1}{h}<1\right)$ induce positive associations $(O R>1)$. Conversely, the opposite outcome of negative associations $(O R<1)$ holds if interactions are synergistic $\left(k>1\right.$ and $\left.\frac{1}{h}>1\right)$. Hence, the correspondence between the $O R$ and $\frac{k}{h}$ is reversed Figure 3.2A and Figure 3.2 $\mathrm{B}$ ).

We can understand this reversion by juxtaposing the two SIRS models. In both models, the $O R$ is computed using the same definition, with in the numerator (N): $\{S S, S R, R S, R R\}$ and $\{I I\}$, and in the denominator (D): $\{I S, I R\}$ and $\{S I, R I\}$. In the direct $S I R S$ model, interactions in acquisition affect the transitions from states in (D) to states in (N), which is reversed in the indirect SIRS model. Correspondingly, in the direct SIRS model, increasing $k$ increases the flow from states in (D) to states in $(\mathrm{N})$ and leads to an increase in the $O R$ (Figure 3.2A dotted line), whereas in the indirect $S I R S$ model, increasing $k$ leads to a decrease in the $O R$ (Figure 3.2A, dashed line). Analogously, increasing $\frac{1}{h}$ leads to an increase in the $O R$ in the direct $S I R S$ model (Figure 3.2 3 , dotted line) and a decrease in the $O R$ in the indirect $S I R S$ model (Figure 3.23, dashed line). Given that $O R=1$ at $k=\frac{1}{h}=1$, inducing cross-immunity by decreasing $k$ and $\frac{1}{h}$ from 1 leads to $O R>1$.

\section{Predicting type replacement in presence of immunity}

In the indirect $S I R S$ model, the $O R$ is not predictive for type replacement due to the reversed correspondence between the $O R$ and $\frac{k}{h}$. In the direct $S I R S$ models, if both mechanisms of interactions are operating in the same direction, that is, either $k>1, \frac{1}{h}>1$ or $k<1, \frac{1}{h}<1$, the outcome of vaccination is also still determined by the value of $\frac{k}{h}$ with respect to 1 . For more complicated situations in which the two mechanisms of interactions operate in opposite directions, that is, either $k<1, \frac{1}{h}>1$ or $k>1, \frac{1}{h}<1$, the outcome of vaccination also depends on the type-specific parameters. For example, if current infections of one type increase the susceptibility for the other type (synergy, $k>1$ ) but accelerate clearance of the other type (competition, $\frac{1}{h}<1$ ), the outcome of vaccination may be $\Phi=-$ while $O R>1$, masking type replacement. InFigure 3.5 this discrepancy is shown by the differences between the $\Phi$-map and the $O R$-map in the upper left and the lower right quadrants.

\section{Discussion}

In this chapter, we studied the usefulness of co-occurrence patterns of pathogen types in cross-sectional prevalence data for predicting type replacement. We confirmed the $O R$ of co-occurrence as an estimator of interactions in acquisition and 


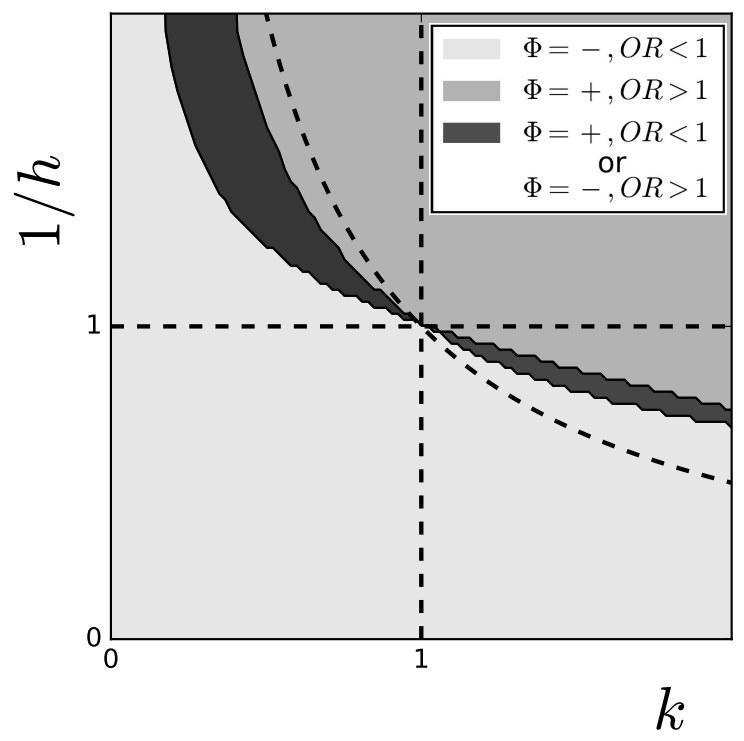

Figure 3.5: The outcome of vaccination and the $O R$ agree when both mechanisms of interactions operate in the same direction (the upper right and lower left quadrants) but may differ when the two mechanisms operate in different directions (the upper left and lower right quadrants). $\Phi=-(+)$ denotes the (non-)occurrence of type replacement by the non-vaccine type. The line $\frac{k}{h}=1$ depicts the boundary between $\Phi=+$ and $\Phi=-$ in the homogeneous SIS model.

clearance in models, either $S I S$ or $S I R S$, with direct interactions. This correspondence between the $O R$ and the interactions is reversed in the setting of a SIRS model with indirect interactions, that is when natural immunity against one type modifies the infection dynamics of another type.

We found that $O R>1$ can be caused by various mechanisms, namely direct synergistic interactions, cross-immunity or confounding due to unobserved common risk factors. As $O R>1$ can be caused by cross-immunity, it cannot preclude type replacement. On the other hand, we confirm $O R<1$ being predictive for type replacement in the setting without immunity, even under confounding by unobserved common risk factors. With immunity, it is predictive for type replacement only if 1) different mechanisms of interactions do not act in different directions; and 2) natural immunity against the vaccine types does not promote infections of the non-vaccine types. Such immunity-induced synergism is rare but has been reported for different strains of the dengue virus in the form of antibody-dependent enhancement [22].

The conditions, identified by our models, under which $O R<1$ is predictive for type replacement may not be generalisable if we depart from the model assumptions we considered. For instance, the predictive power of $O R<1$ for type re- 
placement may disappear under asymmetric interactions among types. Although mild asymmetry (e.g. if one type experiences stronger competition from another type than vice versa), may retain the predictive power, more extreme asymmetry (e.g. if one type is synergistic towards another type from which it experiences competition) may not. Furthermore, we did not consider within-host competition for transmission, which affects the transmission capacity in co-infected relative to singly infected individuals. In addition, natural immunity may result in a mix of direct and indirect interactions, different than in the SIRS models considered here, further complicating the interpretation of the $O R$.

Other model assumptions on contact patterns and transmission may lead to different interpretations of the $O R$. For example, the $S$ usceptible-Inected-RecoveredSusceptible model of Malagón et al. allows simultaneous acquisition when susceptibles are partnered with co-infected individuals [11]. Their model yields $O R>$ 1 even if the type interactions are independent, in contrast to our SIRS models, which find $O R=1$. This bias of the $O R$ under independence arises because simultaneous acquisition is itself a mechanism that enhances the co-occurrence of types.

We assumed cross-sectional prevalence data to be sampled from an epidemiologic equilibrium in which a stable prevalence of infections is maintained. This stationary assumption is reasonable in the pre-vaccination era for endemic pathogens, like S. pneumoniae and HPV, and is commonly assumed in transmission modelling [23]. After the introduction of vaccination, this assumption is violated until the prevalence has re-established at a new equilibrium. When the prevalence oscillates through the years but has a seasonal pattern, other statistical methods using time series to infer type interactions might be more suitable [22]. Furthermore, individuals reach the stationary distribution only after being at risk for some time in practice. The time required to achieve stationary depends on the speed of the transmission process and may differ between pathogens.

For HPV, there have been many studies on pre-vaccination cross-sectional data that used the $O R$ to infer competitive interactions [13-15]. These studies usually adjust for possible unobserved common risk factors by either including individual-specific random effects or by comparing each type-to-type $O R$ to the pooled $O R$. After adjustment, most studies find $O R>1$ or fail to find systematic deviations of the type-to-type $O R$ 's from the pooled $O R$. However, conclusions concerning type replacement should be drawn cautiously in view of alternative explanations for these findings, including cross-immunity between types, which entails a risk of type replacement. Furthermore, adjustment for unobserved common risk factors need not be similar among types. Essentially, previous models account for unobserved risk factors by assuming a random effect that is the same for all pairs of types. Our results show that random effects could be different for each type-to-type $O R$. Whether or not such differences among type-to-type combinations are practically negligible depends on the application. If not, one may 
resolve to random effects models that also account for differences between types [24].

HPV vaccines have demonstrated to be cross-protective for some non-vaccine HPV types that are phylogenetically related to the vaccine types [25-28]. Such cross-protection may substitute the competitive pressure by the vaccine types on the non-vaccine types and counterbalance type replacement. Including crossprotection would alter the outcome of vaccination in our analyses. For some scenarios with $O R<1$ calculated from the pre-vaccination prevalence, type replacement may be mitigated or even prevented by cross-protection if cross-protective efficacy is strong enough. Consequently, while $O R<1$ predicts the potential for type replacement, type replacement need not occur in the presence of vaccine cross-protection.

For other pathogens, it is less common to use the $O R$ of co-occurrence in a cross-sectional setting to study type replacement. We only know of Bogaert et al., who studied the possibility of Staphylococcus aureus replacing $S$. pneumoniae after the introduction of PCV-7 [29]. For S. pneumoniae serotypes, we know of no studies on patterns of co-occurrence in the setting we discuss before PCV-7 was introduced.

We note that our models predict a stable equilibrium frequency distribution of $50 \%-50 \%$ when applied to epidemiologically indistinguishable types. Hence, they are not neutral from a population-genetic point of view as described by Lipsitch et al., who argued that non-neutral models are unsatisfactory in explaining the longterm coexistence of types when the evidence for competition is compelling [30]. Thereupon, various neutral models have been suggested, all assuming a form of competition, for example, by limiting the number of types that a host can carry or by inducing homologous immunity. Yet, a neutral model that intrinsically assumes competition may not be appropriate for developing the framework to test for signs of competition, since it has no natural representation for the absence of competition, in contrast to our ecologically non-neutral models. Furthermore, stable coexistence of $50 \%-50 \%$ frequency is not a problem if one assumes types that are independent of each other. Even if types are epidemiologically indistinguishable (e.g. if they share the same transmission route and have similar infection cycles), it is reasonable that they converge to the same frequency if they are not interfering with each other during infection or transmission.

So far, only a few studies have commented on the validity of using the $O R$ of co-occurrence to predict type replacement. This study is the first to provide conditions under which the $O R$ is an estimator of interactions and under which it is predictive for type replacement. Furthermore, our contribution provides analytical proofs and intuition for earlier findings, such as the manifestation of unobserved confounding and the reversed relationship between the $O R$ and type replacement due to cross-immunity. 
Type replacement following vaccination may have a detrimental impact on public health. Prediction of type replacement based on the $O R$ of co-occurrence in cross-sectional studies has an intuitive appeal, which is mathematically grounded. However, when knowledge of the underlying mechanisms of interactions and the structure of confounding is lacking, observed patterns of co-occurrence allow for various explanations. Hence, the settings in which the $O R$ unambiguously indicates the possibility of type replacement is narrowed down to pathogens in endemic equilibrium, with a well-understood infection cycle and natural immunity. As such, to assess vaccination effects on the prevalence of non-vaccine types, postvaccine surveillance studies remain essential because potential pitfalls in predicting type replacement are pervasive.

\section{References}

[1] O. Balmer and M. Tanner. "Prevalence and implications of multiple-strain infections". Lancet Infect Dis 11.11 (2011), 868-878.

[2] A. R. Mclean. "Vaccination, evolution and changes in the efficacy of vaccines: a theoretical framework". Proc Biol Sci 261.1362 (1995), 389-393.

[3] M. Lipsitch. "Vaccination against colonizing bacteria with multiple serotypes". Proc Natl Acad Sci U S A 94.12 (1997), 6571-6576.

[4] E. H. Elbasha and A. P. Galvani. "Vaccination against multiple HPV types". Math Biosci 197.1 (2005), 88-117.

[5] G. S. Ribeiro, J. N. Reis, S. M. Cordeiro, et al. "Prevention of Haemophilus influenzae type b (Hib) meningitis and emergence of serotype replacement with type a strains after introduction of Hib immunization in Brazil". J Infect Dis 187.1 (2003), 109-116.

[6] L. A. Hicks, L. H. Harrison, B. Flannery, et al. "Incidence of pneumococcal disease due to non-pneumococcal conjugate vaccine (PCV7) serotypes in the United States during the era of widespread PCV7 vaccination, 1998-2004". J Infect Dis 196.9 (2007), 1346-1354.

[7] D. M. Weinberger, R. Malley, and M. Lipsitch. "Serotype replacement in disease after pneumococcal vaccination". Lancet 378.9807 (2011), 1962-1973.

[8] M. Plummer, S. Vaccarella, and S. Franceschi. "Multiple human papillomavirus infections: the exception or the rule? (editorial)". J Infect Dis 203.7 (2011), 891-893.

[9] M. Pons-Salort, A. C. Thiébaut, D. Guillemot, et al. "HPV genotype replacement: too early to tell (correspondence)". Lancet Infect Dis 13.12 (2013), 1012.

[10] D. P. Durham, E. M. Poolman, Y. Ibuka, et al. "Reevaluation of epidemiological data demonstrates that it is consistent with cross-immunity among human papillomavirus types". J Infect Dis 206.8 (2012), 1291-1298.

[11] T. Malagón, P. Lemieux-Mellouki, J. F. Laprise, et al. "Bias due to correlation between times-atrisk for infection in epidemiologic studies measuring biological interactions between sexually transmitted infections: a case study using human papillomavirus type interactions". Am J Epidemiol 184.12 (2016), 873-883.

[12] J. E. Tota, A. V. Ramanakumar, M. Jiang, et al. "Epidemiologic approaches to evaluating the potential for human papillomavirus type replacement postvaccination". Am J Epidemiol 178.4 (2013), 625-634. 
[13] A. F. Rositch, C. Poole, M. G. Hudgens, et al. "Multiple human papillomavirus infections and type competition in men". J Infect Dis 205.1 (2012), 72-81.

[14] A. K. Chaturvedi, H. A. Katki, A. Hildesheim, et al. "Human papillomavirus infection with multiple types: pattern of coinfection and risk of cervical disease". J Infect Dis 203.7 (2011), 910-920.

[15] M. Mollers, H. J. Vriend, M. A. van der Sande, et al. "Population- and type-specific clustering of multiple HPV types across diverse risk populations in the Netherlands". Am J Epidemiol 179.10 (2014), 1236-1246.

[16] M. Pons-Salort, V. Letort, and M. e. a. Favre. "Exploring individual HPV coinfections is essential to predict HPV-vaccination impact on genotype distribution: a model-based approach". Vaccine 31.8 (2013), 1238-1245.

[17] K. Auranen, J. Mehtälä, A. Tanskanen, et al. "Between-strain competition in acquisition and clearance of pneumococcal carriage - epidemiologic evidence from a longitudinal study of day-care children". Am J Epidemiol 171.2 (2010), 169-176.

[18] P. R. Mercer. More calculus of a single variable. Springer, 2014.

[19] P. Lemieux-Mellouki, M. Drolet, J. Brisson, et al. "Assortative mixing as a source of bias in epidemiological studies of sexually transmitted infections: the case of smoking and human papillomavirus". Epidemiol Infect 144.7 (2016), 1490-1499.

[20] P. E. Gravitt. "The known unknowns of HPV natural history". J Clin Invest 121.12 (2011), 45934599.

[21] N. Principi, L. Terranova, A. Zampiero, et al. "Oropharyngeal and nasopharyngeal sampling for the detection of adolescent Streptococcus pneumoniae carriers". J Med Microbiol 63.3 (2014), 393-398.

[22] S. Shrestha, A. A. King, and P. Rohani. "Statistical inference for multi-pathogen systems". PLoS Comput Biol 7.8 (2011), e1002135.

[23] M. J. Keeling and P. Rohani. Modeling infectious diseases in humans and animals. Princeton University Press, 2008.

[24] B. A. Coull and A. Agresti. "Random effects modeling of multiple binomial responses using the multivariate binomial logit-normal distribution". Biometrics 56.1 (2000), 73-80.

[25] T. Malagón, M. Drolet, M. C. Boily, et al. "Cross-protective efficacy of two human papillomavirus vaccines: a systematic review and meta-analysis". Lancet Infect Dis 12.10 (2012), 781-789.

[26] D. Apter, C. M. Wheeler, J. Paavonen, et al. "Efficacy of human papillomavirus 16 and 18 (HPV16/18) AS04-adjuvanted vaccine against cervical infection and precancer in young women: final event-driven analysis of the randomized, double-blind PATRICIA trial". Clin Vaccine Immunol 22.4 (2015), 361-373.

[27] K. Kavanagh, K. G. Pollock, K. Cuschieri, et al. "Changes in the prevalence of human papillomavirus following a national bivalent human papillomavirus vaccination programme in Scotland: a 7-year cross-sectional study". Lancet Infect Dis 17.12 (2017), 1293-1302.

[28] P. J. Woestenberg, A. J. King, B. H. Van Benthem, et al. "Bivalent vaccine effectiveness against type-specific HPV positivity: evidence for cross-protection against oncogenic types among Dutch STI clinic visitors". J Infect Dis 217.2 (2018), 213-222.

[29] D. Bogaert, A. van Belkum, M. Sluijter, et al. "Colonisation by Streptococcus pneumoniae and Staphylococcus aureus in healthy children". Lancet 363.9424 (2004), 1871-1872.

[30] M. Lipsitch, C. Colijn, T. Cohen, et al. "No coexistence for free: neutral null models for multistrain pathogens". Epidemics 1.1 (2009), 2-13. 


\section{Appendices of Chapter 3}

\section{A. The homogeneous $S I S$ model}

\section{A.1. The linear system at the equilibrium}

The equilibrium of the homogeneous $S I S$ model in terms of $\left\{\lambda_{1}, \lambda_{2}, \mu_{1}, \mu_{2}, k, h\right\}$ can be obtained by solving the following linear system.

$$
\left[\begin{array}{l}
0 \\
0 \\
0 \\
0
\end{array}\right]=\left[\begin{array}{cccc}
-\left(\lambda_{1}+\lambda_{2}\right) & \mu_{1} & \mu_{2} & 0 \\
\lambda_{1} & -\left(\mu_{1}+k \lambda_{2}\right) & 0 & h \mu_{2} \\
\lambda_{2} & 0 & -\left(\mu_{2}+k \lambda_{1}\right) & h \mu_{1} \\
0 & k \lambda_{2} & k \lambda_{1} & -h\left(\mu_{1}+\mu_{2}\right)
\end{array}\right]\left[\begin{array}{c}
S S \\
I S \\
S I \\
I I
\end{array}\right]
$$

\section{A.2. An alternative proof of result I}

To better understand how the $O R$ reduces to $\frac{k}{h}$, we provide an alternative proof for result I using the reversibility property of the model. A model is reversible if the net flow between any pair of states is zero, i.e. for any state $A$ and state $B$, the flow from $A$ to $B$ equals the flow from $B$ back to $A$. The flow from $A$ to $B$ is given by "the prevalence in $A$ " times "the transition rate from $A$ to $B$ ", so that we have the following detailed balance equation:

$$
A \cdot q_{A \rightarrow B}=B \cdot q_{B \rightarrow A}
$$

Checking the detailed balance equation for each pair of states in our model verifies its reversibility at the equilibrium. For example, the flow from $S S$ to $I S$ equals the flow from $I S$ back to $S S$, i.e.

$$
S S \cdot q_{S S \rightarrow I S}=\frac{h \mu_{1} \mu_{2} \lambda_{1}}{C}=I S \cdot q_{I S \rightarrow S S}
$$

This detailed balance property links the prevalence of states, which appear in the definition of $O R$, to the interaction parameters, which appear in the definition of the transition rates, so that we have:

$$
\begin{aligned}
O R & =\frac{I I}{S I} / \frac{I S}{S S} \\
& =\frac{q_{S I \rightarrow I I}}{q_{I I \rightarrow S I}} / \frac{q_{S S \rightarrow I S}}{q_{I S \rightarrow S S}} \\
& =\frac{k \lambda_{1}}{h \mu_{1}} / \frac{\lambda_{1}}{\mu_{1}} \\
& =\frac{k}{h}
\end{aligned}
$$

The first equality of Equation (S3.1) shows that the $O R$ is a ratio between two ratios, $\frac{I I}{S I}$ and $\frac{I S}{S S}$. The second equality evokes the reversibility, which translates these two ratios to ratios of transition rates between $\{I I, S I\}$ and between $\{I S, S S\}$. These transition rates then reduce to $\frac{k}{h}$ according to the definitions. The reversibility is key to the correspondence $O R=\frac{k}{h}$, since the rest of the derivation follows according to the definitions. 


\section{B. The heterogeneous $S I S$ model}

\section{B.1. The system of differential equations}

$$
\begin{cases}\frac{d S S(z, t)}{d t} & =-\left(z \lambda_{1}+z \lambda_{2}\right) S S(z, t)+\mu_{1} I S(z, t)+\mu_{2} S I(z, t) \\ \frac{d I S(z, t)}{d t} & =z \lambda_{1} S S(z, t)-\left(\mu_{1}+k z \lambda_{2}\right) I S(z, t)+h \mu_{2} I I(z, t) \\ \frac{d S I(z, t)}{d t} & =z \lambda_{2} S S(z, t)-\left(\mu_{2}+k z \lambda_{1}\right) S I(z, t)+h \mu_{1} I I(z, t) \\ \frac{d I I(z, t)}{d t} & =k z \lambda_{2} I S(z, t)+k z \lambda_{1} S I(z, t)-h\left(\mu_{1}+\mu_{2}\right) I I(z, t)\end{cases}
$$

\section{B.2. The proof of $O R>1$ under independence}

Here, we prove that the crude $O R$ is greater than 1 if the interactions are independent $(k=h=1)$ at the equilibrium. Equivalently, we prove that the observed-to-expected ratio is greater the 1, i.e. $I I>$ $(I S+I I)(S I+I I)$. Let $f(z)$ be the density function of the susceptibility level so that $\int_{0}^{\infty} f(z) d z=1$. For notational convenience, we also define the following normalised quantities for each $z$ :

$$
\begin{aligned}
& \pi_{I I}(z)=I I(z) / f(z) \\
& \pi_{I *}(z)=(I S(z)+I I(z)) / f(z) \\
& \pi_{* I}(z)=(S I(z)+I I(z)) / f(z)
\end{aligned}
$$

To verify $I I>(I S+I I)(S I+I I)$, we expand the two sides of the inequality. The left-hand side can be written as

$$
\begin{aligned}
I I & =\int_{0}^{\infty} I I(z) d z \\
& =\int_{0}^{\infty} \pi_{I I}(z) f(z) d z \\
& =\int_{0}^{\infty} \pi_{I *}(z) \pi_{* I}(z) f(z) d z \\
& =\left(\int_{0}^{\infty} f(z) d z\right)\left(\int_{0}^{\infty} \pi_{I *}(z) \pi_{* I}(z) f(z) d z\right)
\end{aligned}
$$

The third equality is true, since for each value of $z$ the corresponding system of differential equations follows the same structure as one of the homogeneous $S I S$, but with $\lambda_{i}$ being substituted by $z \lambda_{i}$ (compare the system of ODE of the homogeneous SIS model and Equation (S3.2)]. In the homogeneous $S I S$ model, $O R=1$ at $k=h=1$. Hence, for each $z, O R(z)=1$ at $k=h=1$. Equivalently, $\pi_{I I}(z)=\pi_{I *}(z) \pi_{* I}$. Lastly, the fourth equality holds, as $\int_{0}^{\infty} f(z) d z=1$.

The right hand side can be written as

$$
\begin{aligned}
(I S+I I)(S I+I I) & =\left(\int_{0}^{\infty} I S(z)+I I(z) d z\right)\left(\int_{0}^{\infty} S I(z)+I I(z) d z\right) \\
& =\left(\int_{0}^{\infty} \pi_{1}(z) f(z) d z\right)\left(\int_{0}^{\infty} \pi_{2}(z) f(z) d z\right)
\end{aligned}
$$

We have now arrived at the setting in which we can apply the weighted version of Chebyshev's integral inequality. This inequality says that for any continuous function $f(z)>0$ on $[b, c]$, and continuous functions $\pi_{1}(z)$ and $\pi_{2}(z)$ on $[b, c]$ that are both increasing or both decreasing, the following 

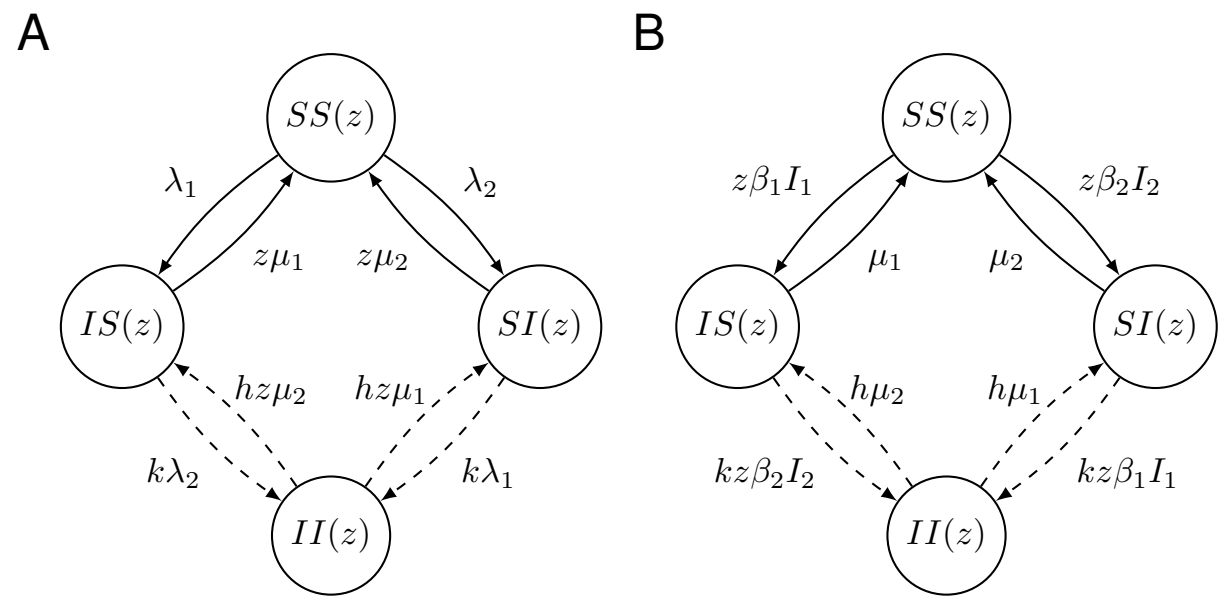

Figure S3.1: The structure of two heterogeneous $S I S$ models for two pathogen types with interactions in acquisition and clearance indicated by dashed arrows. The clearance rate and contact rate are assumed to be heterogeneous in A and B, respectively. $I_{i}$ denotes the proportion of individuals infected by type $i$ throughout the whole population.

holds:

$$
\left(\int_{b}^{c} f(z) d z\right)\left(\int_{b}^{c} \pi_{1}(z) \pi_{2}(z) f(z) d z\right)>\left(\int_{b}^{c} \pi_{1}(z) f(z) d z\right)\left(\int_{b}^{c} \pi_{2}(z) f(z) d z\right)
$$

Hence, the last step is to prove that $\pi_{1}(z)$ and $\pi_{2}(z)$ are both increasing in $z$. For each $z, \pi_{i}(z)$ at the equilibrium satisfies $z \lambda_{i}\left(1-\pi_{i}(z)\right)=\mu_{i} \pi_{i}(z)$, which can be rewritten as $\pi_{i}(z)=\frac{z \lambda_{i}}{\left(z \lambda_{i}+\mu_{i}\right)}$. As $\pi_{i}(z)$ has a strictly positive derivative, i.e. $\frac{d \pi_{i}(z)}{d z}=\frac{\lambda_{i} \mu_{i}}{\left(z \lambda_{i}+\mu_{i}\right)^{2}}>0, \pi_{i}(z)$ is increasing.

Again, in the same manner, heterogeneity in contact rate as given in Figure S3.1 A also yields $\pi_{1}(z)$ and $\pi_{2}(z)$ that are increasing in $z$. Since at the equilibrium, $\pi_{i}(z)$ satisfies $z \beta_{i} I_{i}\left(1-\pi_{i}(z)\right)=\mu_{i} \pi_{i}(z)$, where $z$ is now the varying contact rate and $I_{i}$ is the proportion of individuals infected by type $i$. This equation can be rewritten as $\pi_{i}(z)=\frac{z \beta_{i} I_{i}}{\left(z \beta_{i} I_{i}+\mu_{i}\right)} . I_{i}$ has the same value for all $z$ at the equilibrium. As $\pi_{i}(z)$ has a strictly negative derivative, i.e. $\frac{d \pi_{i}(z)}{d z}=\frac{\beta_{i} I_{i} \mu_{i}}{\left(z \beta_{i} I_{i}+\mu_{i}\right)^{2}}>0, \pi_{i}(z)$ is increasing.

At last, heterogeneity in clearance as given in Figure S3.1 $\beta$ rate yields $\pi_{1}(z)$ and $\pi_{2}(z)$ that are decreasing in $z$. Since at the equilibrium, $\pi_{i}(z)$ satisfies $\lambda_{i}\left(1-\pi_{i}(z)\right)=z \mu_{i} \pi_{i}(z)$, which can be rewritten as $\pi_{i}(z)=\frac{\lambda_{i}}{\left(\lambda_{i}+z \mu_{i}\right)}$. As $\pi_{i}(z)$ has a strictly negative derivative, i.e. $\frac{d \pi_{i}(z)}{d z}=\frac{-\lambda_{i} \mu_{i}}{\left(\lambda_{i}+z \mu_{i}\right)^{2}}<$ $0, \pi_{i}(z)$ is decreasing.

\section{The $S I R S$ models}

\section{C.1. Force of infection}

$$
\left\{\begin{array}{l}
\lambda_{1}=c \beta_{1}(I S+I I+I R) \\
\lambda_{2}=c \beta_{2}(S I+I I+R I)
\end{array}\right.
$$




\section{C.2. Direct interactions}

$$
\begin{cases}\frac{d S S}{d t} & =-\left(\lambda_{1}+\lambda_{2}\right) S S+\gamma_{1} R S+\gamma_{2} S R \\ \frac{d I S}{d t} & =\lambda_{1} S S-\left(\mu_{1}+k \lambda_{2}\right) I S+\gamma_{2} I R \\ \frac{d S I}{d t} & =\lambda_{2} S S-\left(\mu_{2}+k \lambda_{1}\right) S I+\gamma_{1} R I \\ \frac{d I I}{d t} & =\lambda_{2} I S+k \lambda_{1} S I-h\left(\mu_{1}+\mu_{2}\right) I I \\ \frac{d R S}{d t} & =\mu_{1} I S-\left(\lambda_{2}+\gamma_{1}\right) R S+\gamma_{2} R R \\ \frac{d S R}{d t} & =\mu_{2} S I-\left(\lambda_{1}+\gamma_{2}\right) S R+\gamma_{1} R R \\ \frac{d R I}{d t} & =h \mu_{1} I I+\lambda_{2} R S-\left(\mu_{2}+\gamma_{1}\right) R I \\ \frac{d I R}{d t} & =h \mu_{2} I I+\lambda_{1} S R-\left(\mu_{1}+\gamma_{2}\right) I R \\ \frac{d R R}{d t} & =\mu_{2} R I+\mu_{1} I R-\left(\gamma_{1}+\gamma_{2}\right) R R\end{cases}
$$

\section{C.3. Indirect interactions}

$$
\begin{cases}\frac{d S S}{d t} & =-\left(\lambda_{1}+\lambda_{2}\right) S S+\gamma_{1} R S+\gamma_{2} S R \\ \frac{d I S}{d t} & =\lambda_{1} S S-\left(\mu_{1}+\lambda_{2}\right) I S+\gamma_{2} I R \\ \frac{d S I}{d t} & =\lambda_{2} S S-\left(\mu_{2}+\lambda_{1}\right) S I+\gamma_{1} R I \\ \frac{d I I}{d t} & =\lambda_{2} I S+\lambda_{1} S I-\left(\mu_{1}+\mu_{2}\right) I I \\ \frac{d R S}{d t} & =\mu_{1} I S-\left(k \lambda_{2}+\gamma_{1}\right) R S+\gamma_{2} R R \\ \frac{d S R}{d t} & =\mu_{2} S I-\left(k \lambda_{1}+\gamma_{2}\right) S R+\gamma_{1} R R \\ \frac{d R I}{d t} & =\mu_{1} I I+k \lambda_{2} R S-\left(h \mu_{2}+\gamma_{1}\right) R I \\ \frac{d I R}{d t} & =\mu_{2} I I+k \lambda_{1} S R-\left(h \mu_{1}+\gamma_{2}\right) I R \\ \frac{d R R}{d t} & =h \mu_{2} R I+h \mu_{1} I R-\left(\gamma_{1}+\gamma_{2}\right) R R\end{cases}
$$

\section{C.4. The proof of result IV}

We show that the $O R$ is an unbiased estimator of $\frac{1}{h}$ when there are only interactions in clearance but is biased for interactions in acquisition. Together, the $O R$ becomes a biased estimator of $\frac{k}{h}$.

If types interact only through direct interactions in clearance, $O R=\frac{1}{h}$ still holds due to a form of reversibility between $\{S S, S R, R S, R R\},\{I I\},\{S I, R I\}$ and $\{I S, I R\}$. These four groups of states (as indicated by the four grey areas in Figure 3.4 in the main text coincide with the four factors appearing in the definition of the $O R$.

The result $O R=\frac{1}{h}$ can be derived in a similar way as Equation (S3.1) The corresponding detailed balanced equations are

$$
\begin{aligned}
(S S+S R) \cdot q_{S S \rightarrow I S} & =(I S+I R) \cdot q_{I S \rightarrow R S} \\
S I \cdot q_{S I \rightarrow I I} & =I I \cdot q_{I I \rightarrow R I}
\end{aligned}
$$

Each equation of S3.3 corresponds to a pair of groups and describes the flow between them. For instance, the first equation corresponds to the pair $\{S S, S R, R S, R R\}$ and $\{I S, I R\}$. Note that state $R S$ and state $R R$ do not appear in the equation, since they do not have direct transitions to $\{I S, I R\}$. Note also that $S S \cdot q_{S S \rightarrow I S}+S R \cdot q_{S R \rightarrow I R}$ is written as $(S S+S R) \cdot q_{S S \rightarrow I S}$, since $q_{S S \rightarrow I S}$ and $q_{S R \rightarrow I R}$ are both equal to $\lambda_{1}$. 
Furthermore, the following proportionality holds:

$$
\begin{aligned}
S I \cdot p & =S I+R I \\
(S S+S R) \cdot p & =S S+S R+R S+R R
\end{aligned}
$$

S3.4 links $S I$ and $S S+S R$, which appear in the detailed balanced equations in $\mathrm{S3.3}$, to $S I+R I$ and $S S+R S+S R+R R$, which appear in the definition of the $O R$. Hence, using [S3.3] and S3.4], the derivation of $O R=\frac{1}{h}$ goes as follows:

$$
\begin{aligned}
O R & =\frac{I I}{S I \cdot p} / \frac{I S+I R}{(S S+S R) \cdot p} \\
& =\frac{I I}{S I} / \frac{I S+I R}{S S+S R} \\
& =\frac{q_{S I \rightarrow I I}}{q_{I I \rightarrow R I}} / \frac{q_{S S \rightarrow I S}}{q_{I S \rightarrow R S}} \\
& =\frac{\lambda_{1}}{h \mu_{1}} / \frac{\lambda_{1}}{\mu_{1}} \\
& =\frac{1}{h}
\end{aligned}
$$

If types only interact through direct interactions in acquisition, the $O R$ becomes a biased estimator of $k$ unless $k=1 . O R=1$ still constitutes a valid boundary between synergy and competition, however, the $O R$ over-estimates $k$ if $k<1$, and under-estimates $k$ if $k>1$. The bias arises as the reversibility breaks down for $k \neq 1$. The reversibility is violated, since $q_{S S \rightarrow I S}=\lambda_{1}$ and $q_{S R \rightarrow I R}=$ $k \lambda_{1}$, disrupting the proportionality in S3.4 so that the derivation of S3.5] no longer holds.

Since the $O R$ is biased for $k$, it is also biased for the composite of the interaction parameters, $\frac{k}{h}$. 


\section{Chapter 4}

\section{Reconstructing heterogeneous pathogen interactions from co-occurrence data via statistical network infer- ence}

This chapter has been submitted as: I. Man, E. Benincà, M. E. E. Kretzschmar, and J. A. Bogaards. "Reconstructing heterogeneous pathogen interactions from co-occurrence data via statistical network inference". 


\section{Abstract}

Infectious diseases often involve multiple pathogen species or multiple strains of the same pathogen. As such, knowledge of how different pathogen species or pathogen strains interact is key to understand and predict the outcome of interventions that target only a single pathogen or subset of strains involved in disease. While population-level data have been used to infer pathogen strain interactions, most previously used inference methods only consider uniform interactions between all strains or focus on marginal interactions between pairs of strains (without correction for indirect interactions through other strains). Here, we evaluate whether statistical network inference could be useful for reconstructing heterogeneous interaction networks from cross-sectional surveys tracking co-occurrence of multi-strain pathogens. To this end, we applied a suite of network models to data simulating endemic infection states of pathogen strains. Satisfactory performance was demonstrated by unbiased estimation of interaction parameters for large sample sizes. Accurate reconstruction of networks may require regularisation or penalisation for sample size. Of note, performance deteriorated in the presence of host heterogeneity, but this could be overcome by correcting for individual-level risk factors. Our work demonstrates how statistical network inference could prove useful for detecting pathogen interactions and may have implications beyond epidemiology. 


\section{Introduction}

The rapid expansion of sequencing technologies over the last decades has drastically increased our ability to detect within-host pathogen diversity. As a result, it is recognised that infectious diseases often involve multiple pathogen species. For example, diseases may arise from bacterial and viral co-infections [1] 2], or co-infections of multiple strains of the same pathogen species, e.g. serotypes of Streptococcus pneumoniae [3], genotypes of the human papillomavirus [4], or clones of Plasmodium falciparum [5]. It is also increasingly understood that networks of microbial interactions may determine the outcome of preventive or therapeutic interventions (e.g. vaccination, antibiotics, probiotics) in unexpected ways [6-8]. For infectious agents, these interactions between pathogens or pathogen strains can take various forms: infection by one strain can modify susceptibility to subsequent infection by others, and the simultaneous presence of multiple strains can affect the duration, infectiousness as well as severity of infection [9, 10]. Consequently, pathogen interactions may have far-reaching clinical, epidemiological, and eco-evolutionary implications [11-13].

The acknowledgement that pathogen strain interaction is a crucial factor shaping the ecology for a wide number of infectious agents has spurred the development of epidemiological models where hosts can be infected by different pathogens or different strains of the same pathogen [14]. However, inference of interaction parameters in these models is notoriously challenging, especially when pathogen strain interaction is to be extracted from population-level data. The availability of large-scale cross-sectional surveys, detecting the presence of multiple infectious strains simultaneously, has given rise to a wide range of methods for detecting interactions from co-occurrence data [15-20]. These methods rely on identifying deviation from statistical independence, either in the number of pathogen strains occurring in individual hosts, pairwise co-occurrence between any two strains, or the exact combination of co-occurrence.

Clearly, the validity of such statistical methods for detecting pathogen interactions hinges on the assumption that non-interaction implies statistical independence, but statistical associations can be driven by factors other than biological interactions [21]. This point has been made repeatedly by community ecologists, concerned with the possibility to detect interdependence among species from cooccurrence patterns across habitats [22], and has been reiterated by epidemiologists [23]. A common criticism of using statistical associations is that hosts or habitats are not identical, and this may favour the presence of one or another (or both) species irrespective of biological interactions. Opportunely, epidemiological studies on human pathogens typically offer rich meta-data, making it possible (in principle) to correct for host heterogeneity. Moreover, when studying different strains of the same pathogen, one might assume that the same hosts are more likely than others to become infected with all strains, justifying correction for host 
heterogeneity by host-specific covariates in statistical models [23].

So far, many existing methods for detecting multi-strain pathogen interactions are restricted to uniform (or homogeneous) interactions across strains (i.e. all strains under consideration interact with one another in the same manner) or focused on marginal interactions between pairs of pathogen strains. Marginal estimation of pairwise interactions does not necessarily control for indirect interactions through other strains and may therefore be prone to bias, especially when strains interact in a heterogeneous manner. Network inference methods relying on conditional dependency between the co-occurrence of multiple strains might be more suited for detecting heterogeneous pathogen strain interactions (e.g. interactions that are different between each pair of strains) but are not widely considered. Moreover, it is not yet well studied how the performance of these methods might be affected by the presence of host heterogeneity.

The purpose of this paper is two-fold. First, we explore the possibility of detecting heterogeneous pathogen interactions from cross-sectional survey data using statistical network inference methods. For this, we evaluate the performance of a suite of network models in reconstructing simulated networks of pathogen strain interactions for a range of epidemiological and network settings. Second, we investigate the robustness of these methods to settings with host heterogeneity that is relevant for pathogen spread. Here, we focus on the reconstruction of interaction networks of pathogen strains, but the methodology is applicable to cross-sectional surveys tracking co-occurrence of potentially interacting pathogen species and may have implications beyond epidemiology.

\section{Results}

In order to evaluate the performance of various network inference methods, we simulated random interaction networks with up to 10 pathogen strains, which describe whether and to what extent the strains interact in an epidemiological model. Within each network, the presence of interaction between each pair of strains $i$ and $j$ was established with connection probability $\sigma$. Given that interaction between a pair was established, the strength of interaction, indicated by the value of $x_{i j}$, was drawn uniformly from the range $[-\theta, \theta]$, where $\theta$ is a positive number. Values of $x_{i j}$ in the range $[-\theta, 0)$ and $(0, \theta]$ correspond to competitive and mutualistic interactions, respectively, and the greater the value diverges from zero, the stronger the interaction becomes.

Each network generated as such was then used to parametrise a $S$ usceptibleInfected-Susceptible $(S I S)$ epidemiological model [8]. In the model, $\exp \left(x_{i j}\right)$ was used as the hazard ratio that determines how the presence of strain $i$ in a host alters the host's hazards to acquire or clear strain $j$, and vice versa. See Section Material and methods for the exact description of how parameter $x_{i j}$ is utilised 
in the parametrisation of the epidemiological model. After parametrisation, the steady state prevalence of the epidemiological model was obtained and used to sample random cross-sectional datasets with the number of observations being $100,1.000,10.000$, or 100.000 . These datasets were then used to reconstruct the interaction networks. See Figure S4.1 for a schematic representation of the entire data generation process.

The set of network inference methods that we considered were the Ising model, graphical modelling approaches, and generalised estimating equations (GEE) [2426]. For the Ising model and GEE, model selection was done based lasso (least absolute shrinkage and selection operator) and Wald tests with false discovery rate control, respectively. Graphical model selection was performed either in a forward or a backward fashion (i.e. by iteratively growing or shrinking networks), using either AIC or BIC as selection criterion.

In the base-case setting, we considered 500 random networks with 5 strains, each generated with connection probability $\sigma=0.25$ and a range of interaction parameters of $[-\theta, \theta]=[-\log 3, \log 3]$. In the corresponding epidemiological model, we considered interaction in acquisition only (i.e. no interaction in clearance), a population without host heterogeneity, and type-specific basic reproduction numbers $R_{0, i}$ in the range $[1.5,2]$.

In the base-case setting, visual inspection showed satisfactory concordance between the estimated networks and true networks. This is illustrated in Figure 4.1 which also shows some differences between network inference methods. In order to compare the different methods systematically, we assessed various performance measures, including sensitivity and specificity for indicating the presence (or absence) of pairwise interactions, i.e. the proportions of truly present (or truly absent) interactions that are correctly identified in the reconstructed networks. In addition, we assessed the F1-score, which summarises sensitivity and positive predictive value (PPV, i.e. the proportion of identified interactions that are truly present) in the form of their harmonic mean.

\section{Trade-off between sensitivity and specificity}

Network reconstruction improved with the number of observations according to almost all assessed performance measures for all considered methods (Figure 4.2AD, Table S4.1. Interactions were recovered with almost $100 \%$ sensitivity at 100,000 observations (Figure 4.2A). Optimal specificity was attained already at small sample sizes in graphical modelling approaches with BIC-based, whereas specificity remained at a more or less constant suboptimal level in graphical modelling approaches with AIC-based selection (Figure 4.2D). GEE was good at small sample sizes but was the only method considered with slightly deteriorating performance with increasing sample size notwithstanding correction for multiple testing (Figure 4.2 3 -D). Overall, methods with less optimal specificity showed better sensi- 

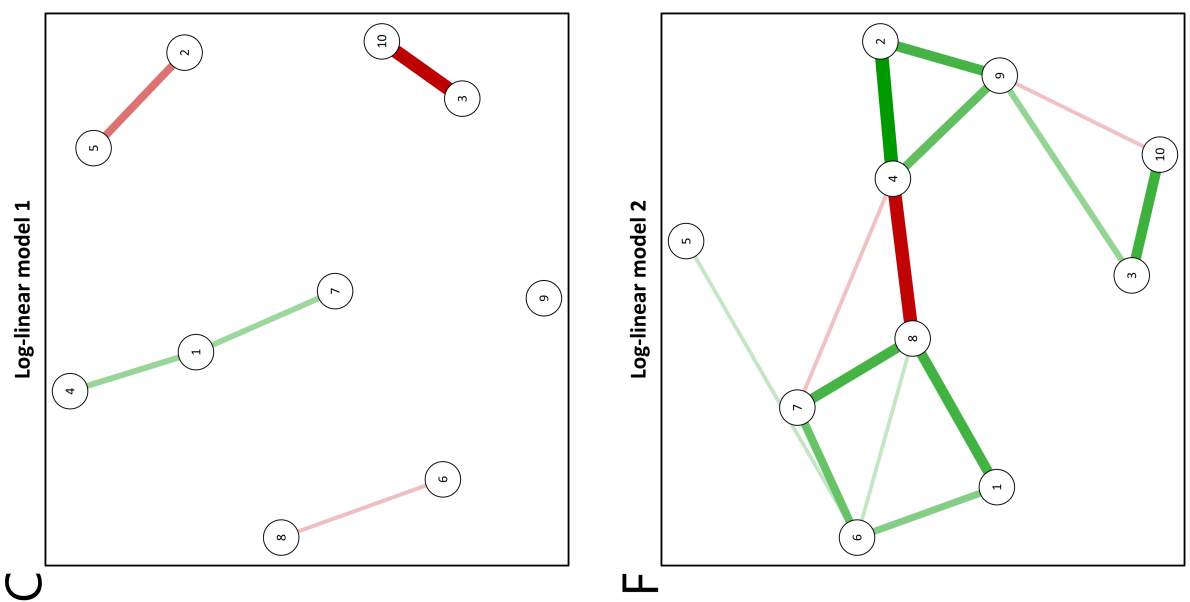

ப
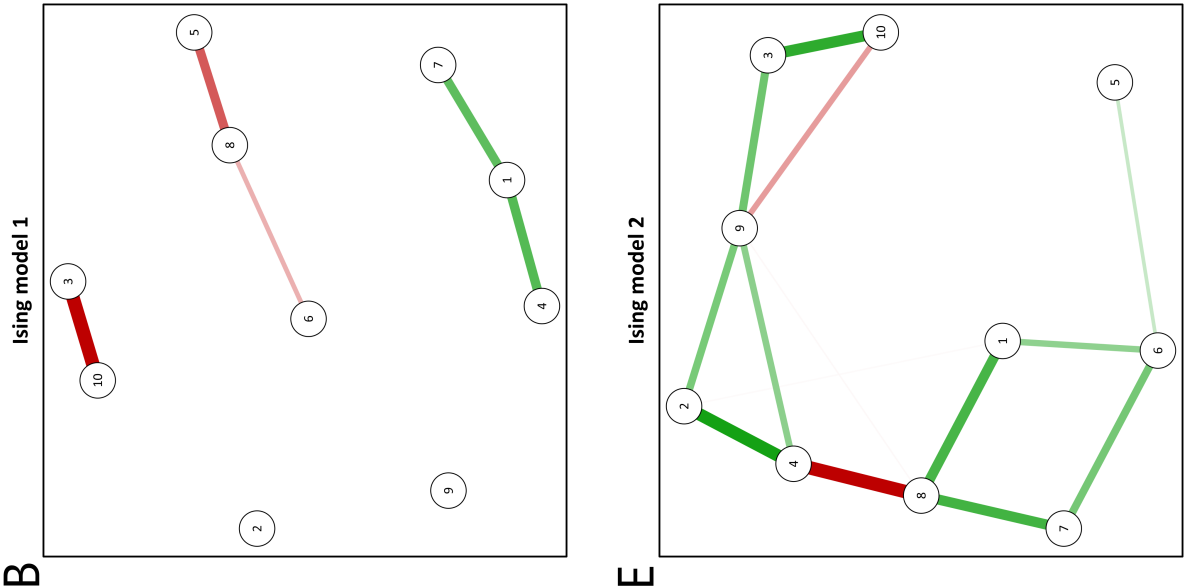

山
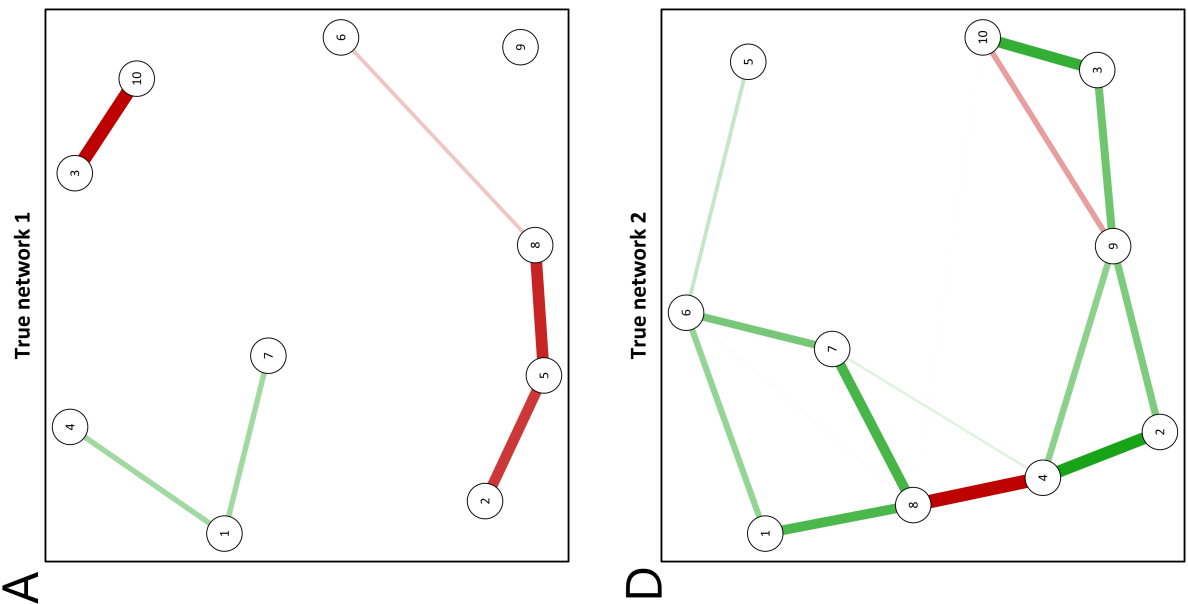

Figure 4.1: Examples of networks with $n=10$ strains in the simulation study. Two random networks generated with connection probability $\sigma=0.25$ and interaction strengths $x_{i j}$ drawn from $[-\theta, \theta]=[-\log 3, \log 3]$ (A and D); Estimated networks from co-occurrence in 100,000 observations according to the regularised Ising mode ( $\mathrm{B}$ and $\mathrm{E})$; and according to a graphical model using backward model selection by BIC ( $\mathrm{C}$ and $\mathrm{F})$. Strength and type of interactions (mutualistic versus competitive) are indicated by the thickness and colour (green versus red) of edges, respectively. 
tivity, showcasing a trade-off between sensitivity and specificity. Finally, the Ising model and the graphical modelling approaches with BIC-based selection were able to achieve relatively high values of sensitivity and specificity at high sample sizes, which resulted in near-optimal F1-scores. (Figure 4.23,C).

\section{Unbiased estimation of the strength of interaction}

In addition, we assessed the quantitative agreement between the estimated and the true interaction parameter $x_{i j}$, which indicates the strength of interaction, based on Pearson correlation and root-mean-square deviation. Average Pearson correlations between the true and estimated interactions were high for all methods with 1,000 observations or more (Figure 4.2E). Under the smallest sample size involving only 100 observations, fewer interactions were recovered, and the estimated interaction strength deviated somewhat from the true parameters, but even in this case, the average correlation between true and estimated interaction strength remained substantial, especially under GEE or regularised Ising estimation (Table S4.1. Moreover, while correlations between true and estimated interactions reached a plateau above 1,000 observations, accuracy continued to improve with increasing sample size, as verified by steadily decreasing root-mean-square deviations for all methods considered, showcasing asymptotically unbiased estimation (Figure 4.2F).

\section{Performance under alternative epidemiological settings and network configurations}

In a series of sensitivity analyses, we modified the base-case setting to alternative epidemiological settings or network configurations: inclusion of interaction in clearance, lower basic reproduction numbers $R_{0, i} \in[1,1.5]$, stronger interactions $x_{i j} \in[-\log 10, \log 10]$, higher connection probabilities $\sigma=0.5$, larger networks by increasing the number of strains to 10 , or larger networks by connecting two sub-networks of 5 strains.

Network inference was not affected by including interactions in clearance relative to the base-case setting. In this setting, F1-scores remained the same for all methods considered (compare pink dots to black lines in Figure 4.3). This implies that the combined effect of interactions in acquisition and clearance was well captured by the estimated interaction parameters. Specifically, the ratio of the interaction parameters in acquisition and clearance was well estimated.

Looking across all alternative settings, regularised Ising, GEE estimation and BIC-graphical modelling approaches maintained the high performance of the basecase analysis when the sample size was 10,000 or larger (Figure 4.3A,B,C,E). For these methods, when the sample size was 1,000 or smaller, performance stayed more or less the same with higher connection probability or lower reproduction 

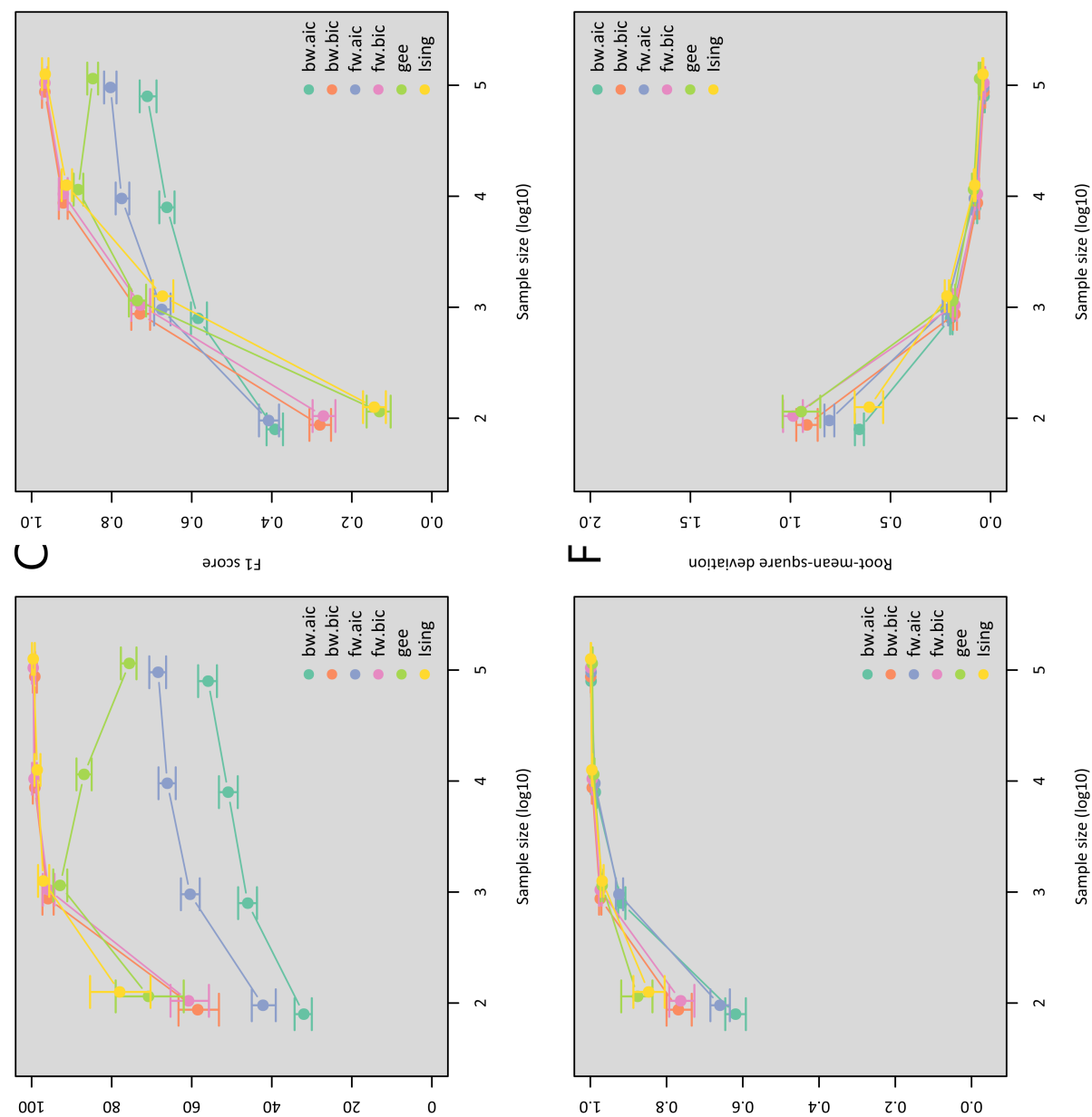

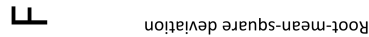

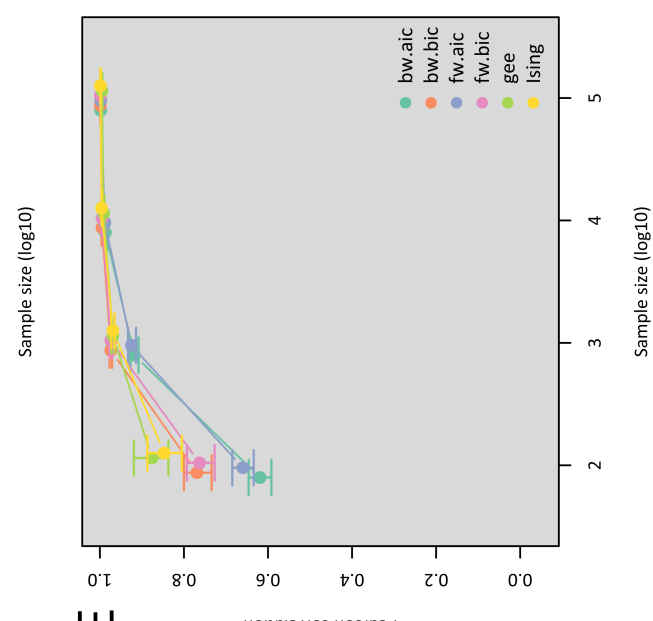

(\%) $\wedge d d$

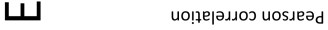
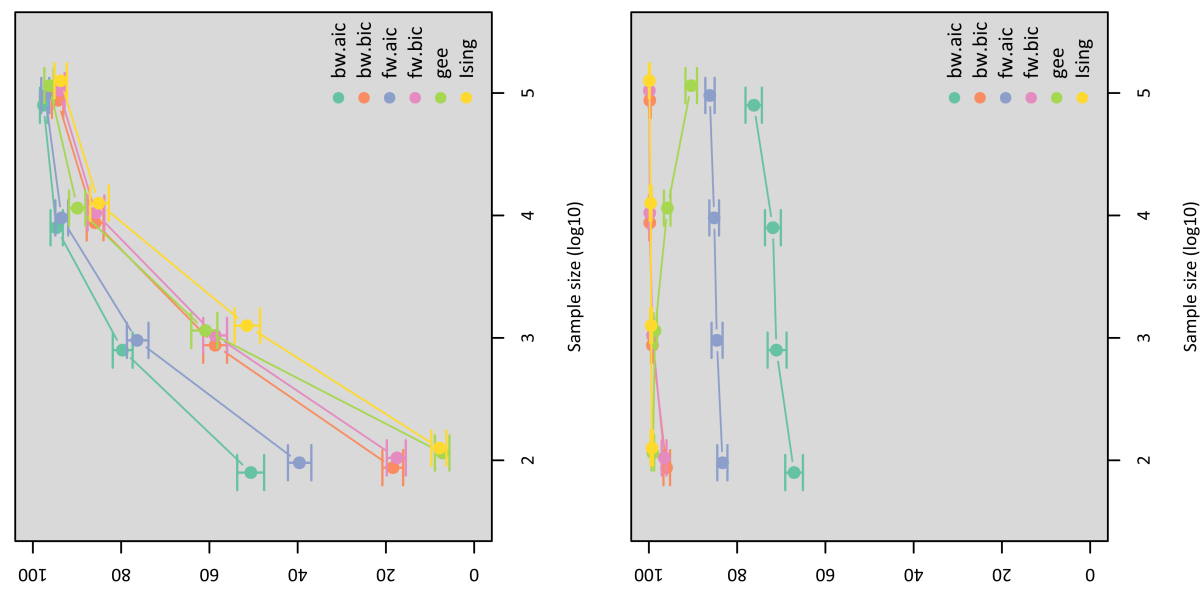

(\%) Kł!n!!!!suəS

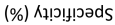

Figure 4.2: Performance measures of statistical network inference in the base-case analysis. Several measures have been calculated to assess the performance of the statistical network inference as a function of the sample size for the base-case analysis. A) Sensitivity; B) Positive predictive value (PPV); C) F1 score; D) Specificity; E) Pearson correlation; F) Root-mean-square deviation. In the base-case analysis, interactions between strains were generated with connection probability $\sigma=0.25$, interaction strengths $x_{i j}$ indicating interaction in acquisition drawn from $[-\log 3, \log 3]$, and basic reproduction numbers randomly drawn from $[1.5,2]$. All methods were evaluated at sample sizes of 100,1,000,10,000 and 100,000 observations, but x-axis coordinates are slightly jittered to improve visualization. Abbreviations: bw.aic (turquoise): graphical model with backward AIC selection; bw.bic (red): graphical model with backward BIC selection; fw.aic (purple): graphical model with forward AIC selection; fw.bic (pink): graphical model with forward BIC selection; gee (green): generalised estimating equations; Ising (yellow): lsing model. 


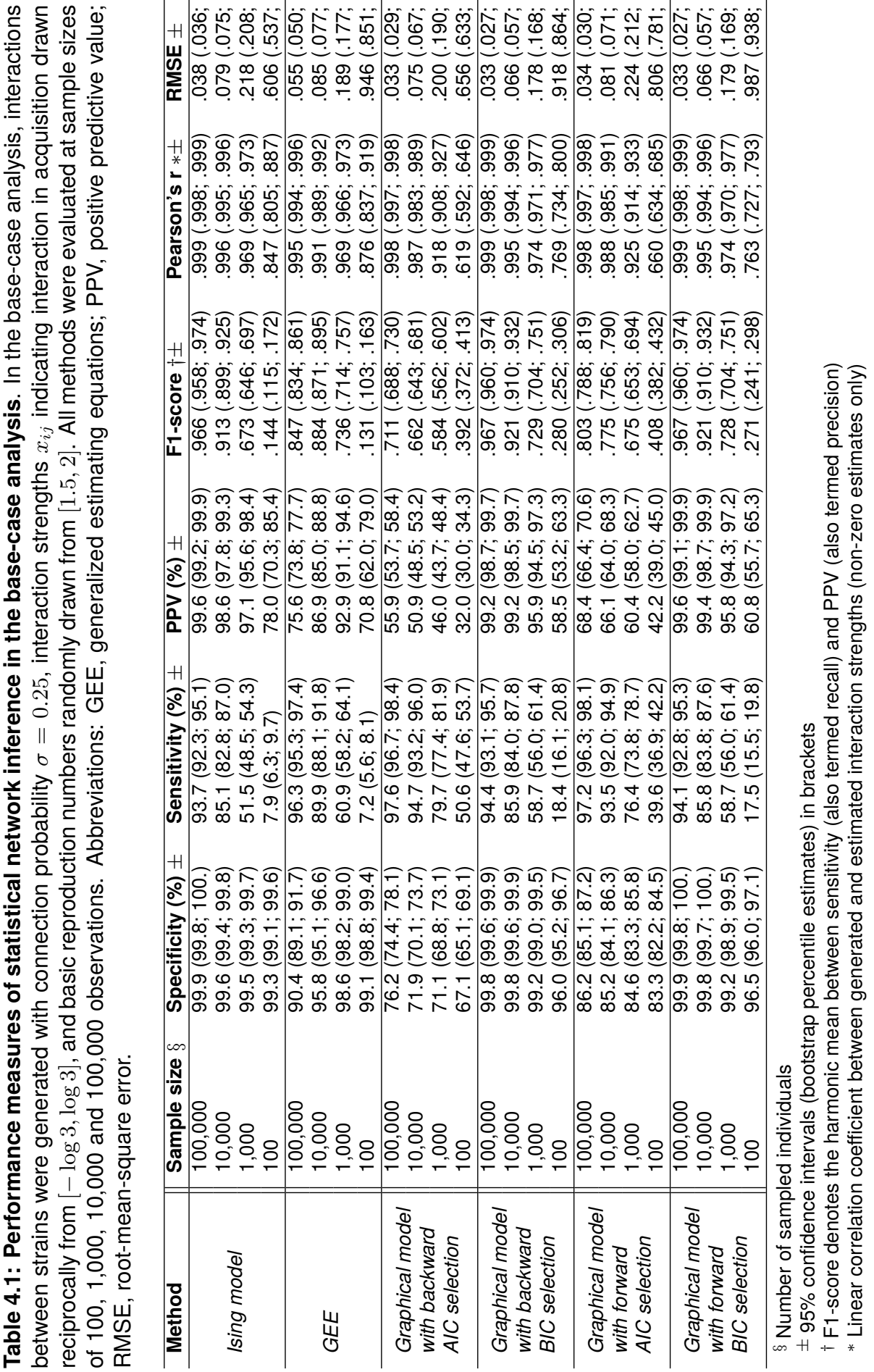


numbers (compare red and turquoise dots to black lines), improved with stronger interactions (compare purple dots to black lines) but slightly deteriorated when considering larger networks of 10 strains (compare green and yellow dots to black lines).

As for the AIC-based graphical modelling approaches, the performance diverged more from that of the base-case setting (Figure 4.3),F). The performance improved substantially under higher connection probabilities (compare red dots to black lines). However, it suffered also more when networks were larger (compare green and yellow dots to black lines). These patterns are linked to the high sensitivity and poor specificity of the AIC-based approaches (as shown in the base-case analysis).

\section{Robustness to host heterogeneity}

Finally, we tested the robustness of the network inference methods to host heterogeneity in contact rate relevant to pathogen spread. The host population was divided into two interconnected sub-populations: one with high and one with low contact rates. Network inference strongly deteriorated in the presence of host heterogeneity as compared to the base-case analysis when heterogeneity was not corrected for (compare turquoise dots to black lines in Figure 4.4, compare $\mathrm{D}$ to $A$ in Figure 4.5. For all methods considered, F1-scores did not improve with increasing sample size. Similarly, root-mean-square deviation remained high at large sample sizes (Table S1). These findings show a consistent bias towards positive associations in uncorrected analyses (Figure S4.2).

Network inference regained good performance if host heterogeneity was corrected for by performing stratified analyses based on the risk variable indicating membership to either sub-population (compare purple and pink dots to black lines in Figure 4.4, compare $B$ and $C$ to $A$ in Figure 4.5. At moderate sample sizes (1.000 and 10.000), performance was somewhat better in subgroup analyses on high-risk individuals, likely due to increased statistical power at the higher prevalence of infection. The alternative correction approach by adding the risk variable as an extra node to the network, representing elevated infection risk, also performed well (compare red to turquoise in Figure 4.4). Graphically, the risk variable can be represented as a central node equally connected to all other nodes representing carriage of strains (Figure 4.5E). This dependency illustrates that elevated infection risk is associated with positivity for each strain. After filtering out the risk variable, the strain-specific interaction network is retained (Figure 4.5F) 

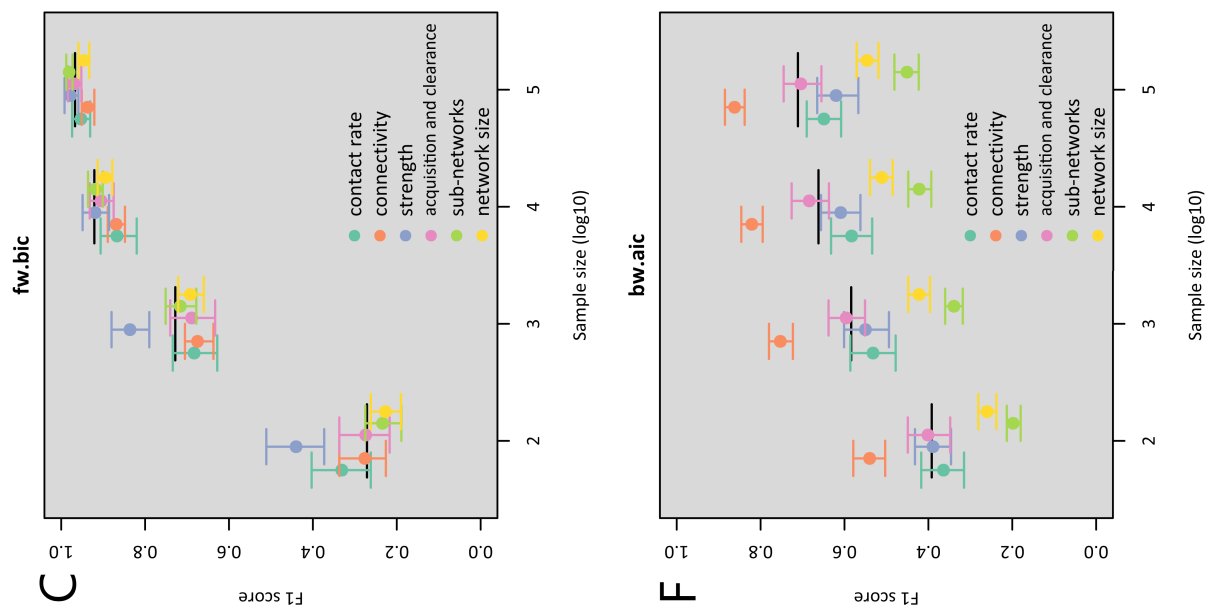

느논
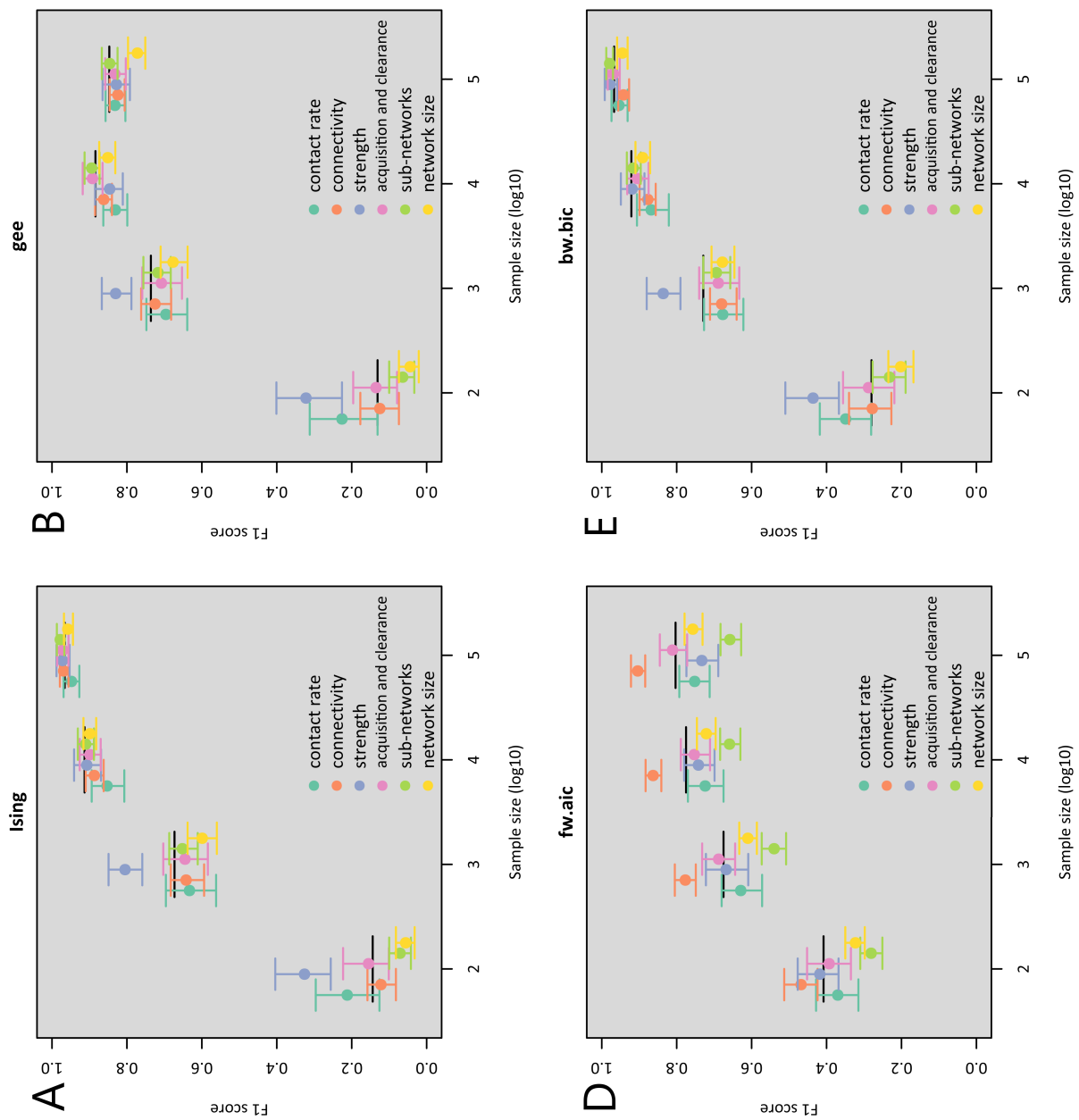

Figure 4.3: F1-scores of statistical network inference methods in the sensitivity analyses. The performance of different network inference methods expressed by F1-score as a function of the sample size. A) Ising model; B) generalised estimating equations; and graphical models with C) forward BIC selection; D) forward AIC selection; E) backward BIC selection; F) backward AIC selection. The alternative settings considered in the sensitivity analyses were: acquisition and clearance (pink): interaction strengths $x_{i j}$ including both interactions in acquisition and clearance; reproduction number (turquoise): lower basic reproduction numbers from the range $[1,1.5]$ instead of $[1.5,2]$; strength (purple): strong interaction strengths $x_{i j}$ drawn from $[-\log 10, \log 10]$ instead of $[-\log 3, \log 3]$; network size (yellow): larger networks with 10 strains; sub-networks (green): larger networks with 10 strains created by collating sub-networks of 5 strains; connection probability (red): higher connection probability $\sigma$ being 0.5 instead of 0.25 . Performance of the base-case analysis is given by black horizontal lines. 

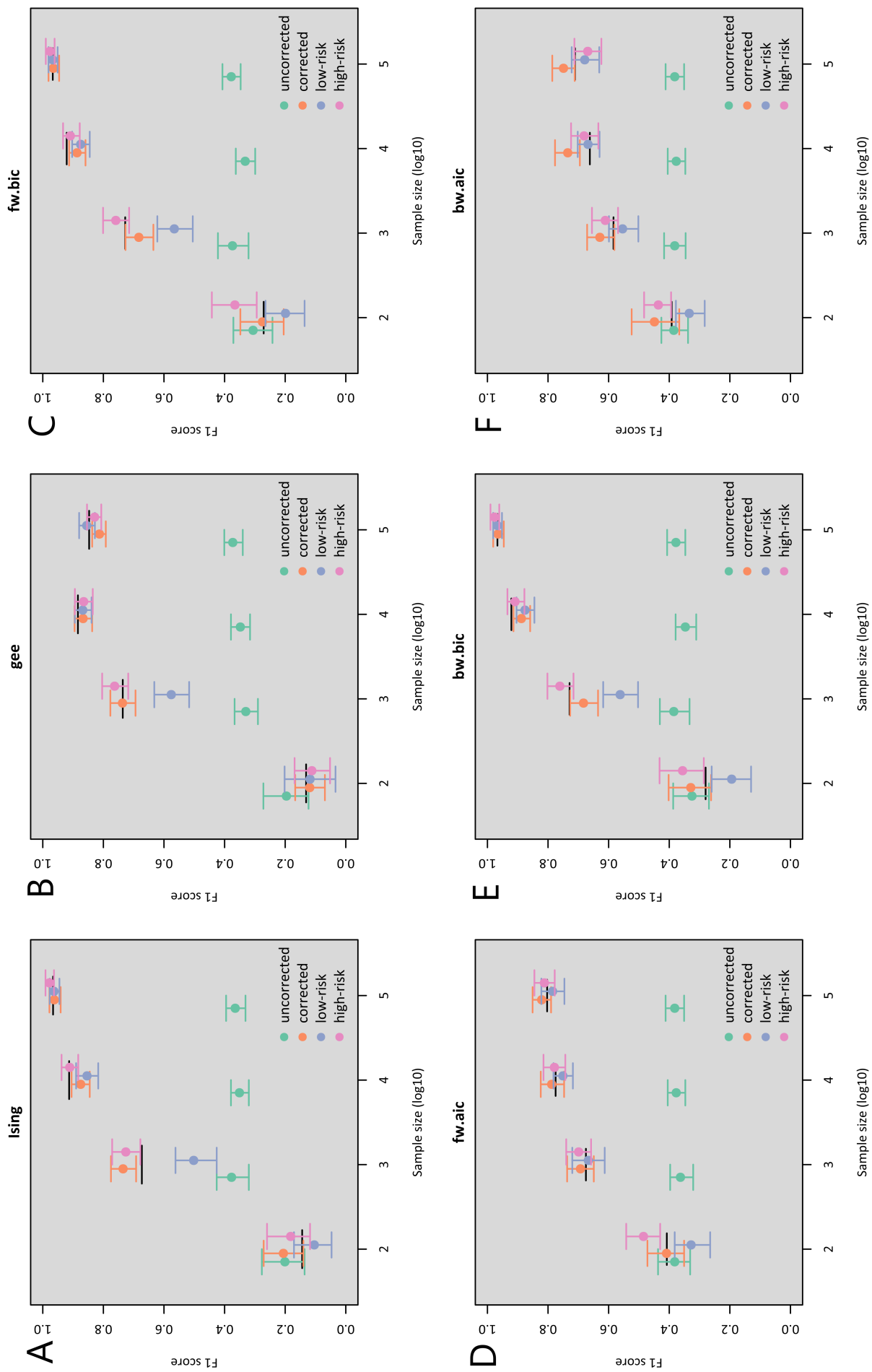

Figure 4.4: F1-scores of statistical network inference under host heterogeneity. The performance of the different inference methods evaluated under the setting with host heterogeneity as a function of the sample size. A) Ising model; B) generalised estimating equations; and graphical models with C) forward BIC selection; D) forward AIC selection; E) backward BIC selection; F) backward AIC selection. Similar epidemiological models are used as in Figure 4.2 but with two sub-populations of hosts. Average contact rate is the same as in the base-case analysis, but $80 \%$ of hosts is assumed to have below-average contacts and $20 \%$ above-average contacts (coefficient of variation: $80 \%$ ). Mixing between sub-populations occurred pseudo-assortatively (assortivity fraction: $50 \%$ ). Performance is investigated in following ways: uncorrected: based on representatively sampled individuals from the total population without correction for contact rate; corrected: idem but with correction for contact rate; low-risk: (stratified) analysis on individuals sampled from sub-population with low contact rate only; high-risk: (stratified) analysis on individuals sampled from sub-population with high contact rate only. 

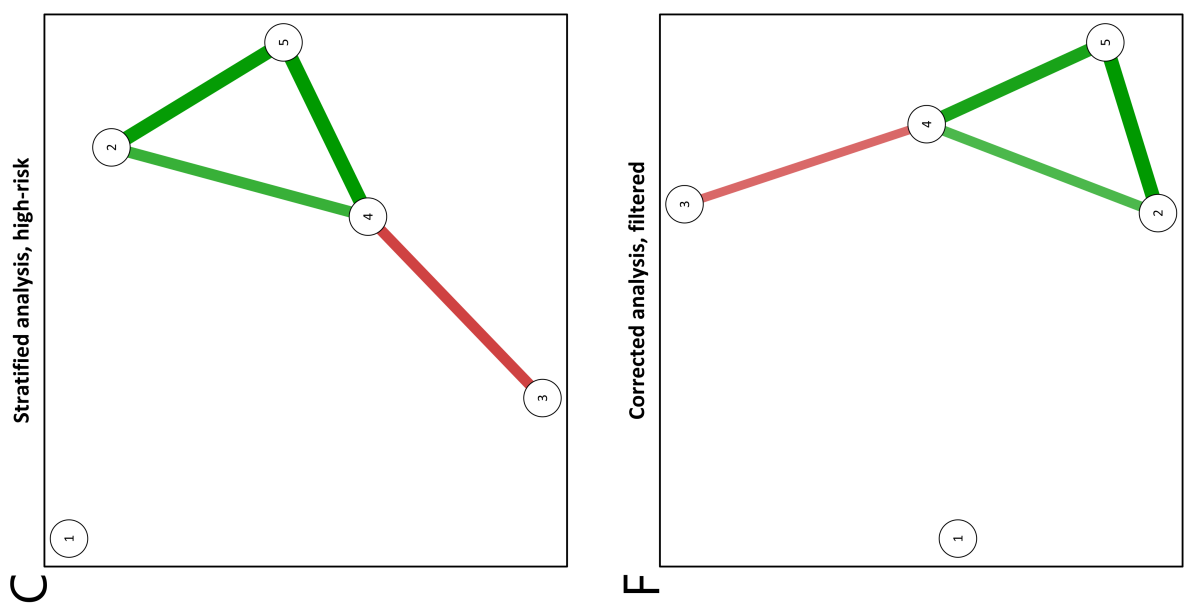

ㄴ.
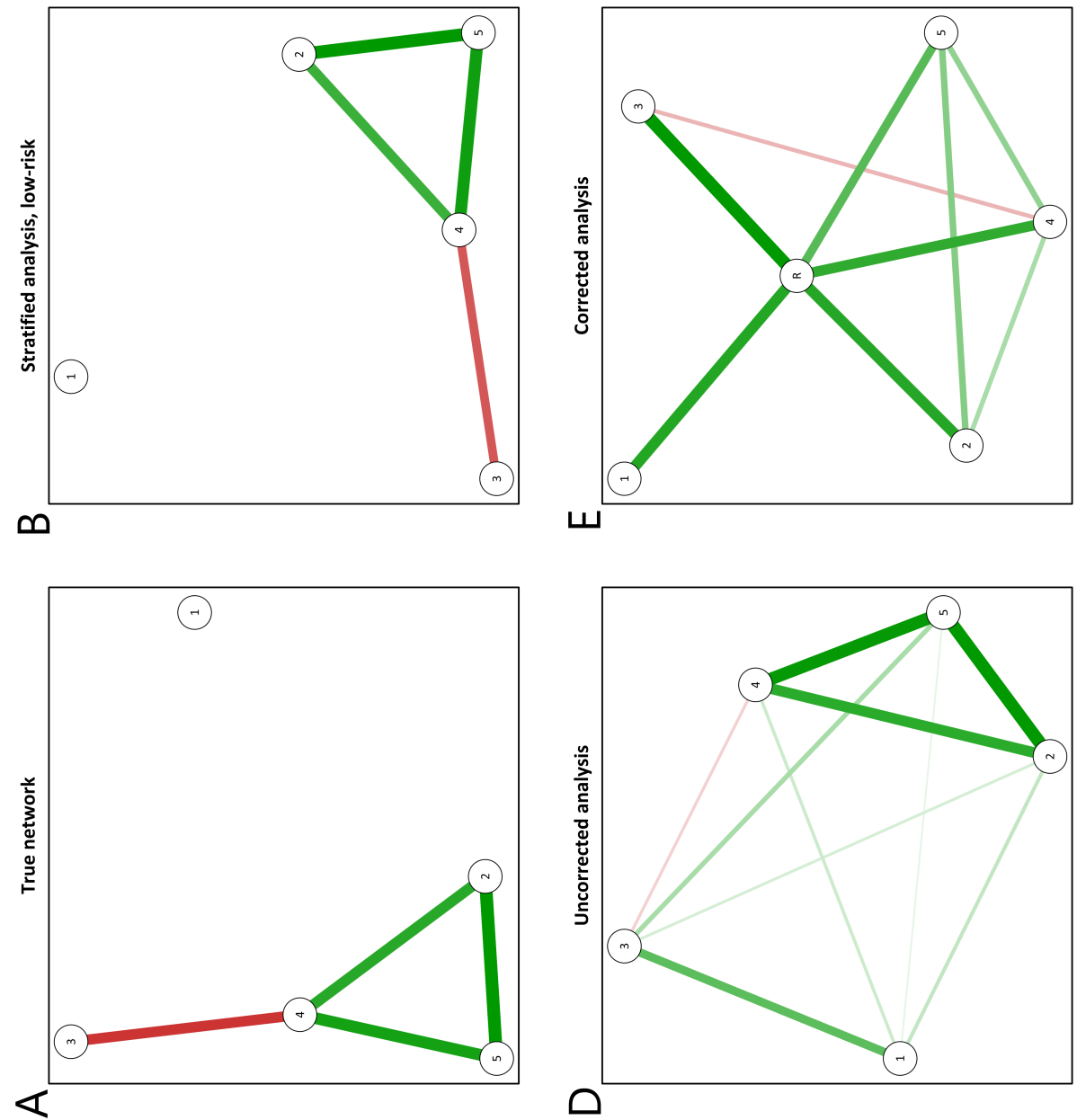

Figure 4.5: An example of network reconstruction under host heterogeneity. The true random network was generated with connection probability $\sigma=0.25$, interaction strengths drawn from $[-\log 3, \log 3]$, and basic reproduction numbers randomly drawn from $[1.5,2](\mathrm{A})$. Estimated networks were obtained using the regularised Ising model from a dataset with 10,000 individuals in a stratified analysis among low-risk individuals only (B), high-risk individuals only (C), among a representative sample of the total population without correction (D), or with correction by augmenting the network with an extra node $\mathrm{R}$, indicating membership to either sub-population (E). The filtered network $(F)$ is obtained from $(E)$ by omitting node $R$ and the corresponding edges. Strength and type of interactions (mutualistic versus competitive) are indicated by the thickness and colour (green versus red) of edges, respectively. 


\section{Discussion}

In this paper, we evaluated whether heterogeneous pathogen strain interactions can be recovered from cross-sectional surveys tracking co-occurrence of multistrain

pathogens via statistical network inference. Using simulated data, we demonstrated unbiased estimation of pairwise interactions between multiple infectious strains, appropriately correcting for indirect interactions through other strains. Performance of network inference was shown to be strongly influenced by host heterogeneity, but we also demonstrated how this can be overcome by correcting for individual risk factors common to all pathogen strains.

Conceivably, the nature and strength of multi-strain interactions may indeed be heterogeneous, as these are modulated by within-host niche differentiation. Such differentiation may arise from slight differences in host-cell tropism, as e.g. described for oncogenic HPV infection, where genotypes of alpha-7 and alpha-9 species have distinct preferences for glandular and squamous epithelium, respectively [4 27]. Moreover, if multi-strain interactions are mediated by within-host immune responses, as hypothesised for different pneumococcal serotypes, the strength of interaction may depend on the antigenic overlap between strains [3]. To uncover heterogeneous interactions, it is essential to analyse datasets where the exact combination of strains in each host is recorded. Previous endeavours to infer competitive interactions from such genotype combination data found that they only performed satisfactorily in datasets where competitive interactions are particularly strong and that accounting for host behavioural heterogeneity is more important than adding additional information via the genotype combinations 20 21). The importance of accounting for host heterogeneity is reiterated in our analysis, but we also demonstrate that network inference is well suited to detect small interactions at large enough sample sizes, provided that the mechanisms of interaction can be inferred from conditional independence structures.

Our results were obtained under a particular epidemiological model and under a steady state assumption, with between-strain interactions modelled in acquisition and clearance. We have previously shown that the odds ratio of co-occurrence in a two-strain version of this model is an exact estimator of the composite of interaction parameters defined accordingly [28]. The results of the present study should be viewed as an extension of that result to systems with an arbitrary number of strains. Network models are well suited to capture conditional independence between multiple strains, providing a clear advantage over marginal estimation of pairwise interactions as these may be confounded through indirect interactions with other strains. While we showed excellent performance of the network inference methods in this respect, we cannot ensure good performance under epidemiological models other than the one we considered. For instance, if strains interact through natural cross-immunity that is long-lasting, infections with differ- 
ent strains may be positively associated (i.e. as given by odds ratios greater than one) and co-occurrence patterns of current infection are no longer informative of their interaction structure [28]. Likewise, strains may interact through modification of viral or bacterial load during co-colonization, rather than through modification of acquisition or clearance. Although such interactions are better captured by records of viral or bacterial load, they are likely correlated with interactions in acquisition and clearance. Hence, network inference based on co-occurrence may retain good performance even in such situations. More extensive simulations are needed to demonstrate the robustness of the network inference methods to variations in model specification. In general, more complex mechanisms of interactions may be better identifiable from individual- or population-level longitudinal studies than cross-sectional surveys [29, 30].

Likewise, the performance of network inference may depend on the way that interactions between multiple strains combine during co-infection. Previously, we demonstrated that predictors of strain-replacement in the wake of vaccination against a subset of pathogen strains, perform best when interactions in acquisition and clearance combine multiplicatively [8]. We envisage that reconstruction of heterogeneous strain interaction networks from co-occurrence data becomes more complicated if interactions combine otherwise than multiplicatively, e.g. if the strength of interaction is not affected by the multiplicity of infection. It should be noted that estimates obtained under GEE or Ising models are also marginal in this sense, as they only consider pairwise interactions and leave possible higherorder interactions unspecified. Conversely, graphical log-linear models implicitly incorporate a three-way interaction whenever three strains are graphically connected, allowing pairwise interactions to be modulated by a third strain [25]. The suitability of either approach is determined by the likelihood of higher-order interactions being present in a particular system, and by the quality and resolution of the available data.

While we did not directly compare the methods applied here to previous approaches, we envisage that the comparatively good performance of statistical network inference derives from their rigour and computational efficiency. These methods bypass the specification of explicit models for interacting strain dynamics and instead capitalise on the implicit relationship between the interaction parameters and the expected proportions of the host population that carry different combinations of infectious strains. Consistency of methods depends on whether the underlying interaction mechanisms are appropriately captured, but the same applies to explicit epidemiological modelling of co-infections. Both approaches may suffer from model misspecification, but if approximations hold, statistical network inference provides a particularly powerful tool, as shown here.

All network modelling approaches considered in this paper show unbiased estimation of interaction parameters, evidenced by diminishing RMSD for increasing sample size. However, we found that different methods excel in different as- 
pects of estimation, with a general trade-off between non-discovery and false discovery. Network structure was only captured appropriately when applying some form of regularization (as in the Ising model) or penalizing for sample size (as in BIC-based log-linear model selection). In comparison, GEE or AIC-based model selection suffered from poor specificity, yielding comparatively low PPV at large sample sizes. Even so, these methods exhibited negligible RMSD, demonstrating that false discovery at large sample sizes predominantly pertains to supposedly small interaction strengths. Penalization ensures that very weak connections are set to zero; hence, the appropriateness of using AIC- or BIC-based model selection depends on the sparsity of the true interaction network being analysed. In our simulations, the sparsity of the networks used to generate co-occurrence data was controlled by fixing the rate of true zeroes in the network. The extent to which true independence holds in reality should determine which class of network inference methods is to be preferred [31]. From a practical point of view, however, the important interactions were almost always correctly identified, also at limited sample sizes.

Finally, we show that a regression framework familiar to most epidemiologists, i.e. GEE, performs satisfactorily in estimating pairwise interactions. The GEE regression framework has the additional benefit that it offers a flexible way of separating individual risk factors common to all strains, from interactions between any two strains [23]. This framework has been employed before to study clustering patterns of HPV genotypes across risk populations, with correction for known risk factors [18]. While correction for individual-level characteristics is also possible in other methods, as demonstrated in the present study, most other graphical modelling approaches rely on the inclusion of risk factors as binary nodes to the network. This clearly limits the practical ability to correct for various sources of host heterogeneity, which is more naturally accommodated in a regression framework [26. 32]. Compared to other graphical modelling approaches, the GEE framework is not scalable to situations where the number of strains exceeds the number of observations. However, for most epidemiological studies this does not pose a real problem.

To summarise, we have demonstrated how interactions between multiple pathogen strains might be estimated from cross-sectional surveys, detecting the presence of multiple infectious strains at once. We illustrated this by applying statistical network models, that properly account for conditional independence between strains and are able to efficiently and consistently estimate heterogeneous interactions. Our work demonstrates how widely available multivariate methods may be used to identify between-strain interactions from readily available data. 


\section{Material and methods}

\section{Multi-strain epidemiological model}

The epidemiological model we used for simulation was previously introduced to assess the outcome of vaccination against a subset of pathogen strains present in a host population [8]. In short, this model allows for an arbitrary number of infectious strains to circulate, assuming Susceptible-Infected-Susceptible $(S I S)$ dynamics with regard to each of the individual strains. It applies to pathogens for which naturally acquired immunity is limited so that reinfection is possible [33], as for instance could be the case for infection with human papillomavirus or with Streptococcus pneumoniae. For a pathogen with $n$ strains, the model state space $\mathcal{S}$ consists of $2 n$ infection states, each denoted by the set of strains the host is infected with. See Figure S4.3 for a schematic representation of the possible infection states with $n=3$. A system of ordinary differential equations describes how the proportions of individuals in each of the model states evolve over time. The equation for the change in prevalence $N_{X}$ of any state $X \in \mathcal{S}$ is generally given by

$$
\begin{aligned}
\frac{d N_{X}}{d t}= & \sum_{i \in X} N_{X \backslash\{i\}} q_{X \backslash\{i\} \rightarrow X}-\sum_{i \in X} N_{X} q_{X \rightarrow X \backslash\{i\}} \\
& -\sum_{i \notin X} N_{X} q_{X \rightarrow X \cup\{i\}}+\sum_{i \notin X} N_{X \cup\{i\}} q_{X \cup\{i\} \rightarrow X} .
\end{aligned}
$$

The four groups of terms on the right-hand side, moving from left to right, correspond to flows of individuals into or out of state $X$ due to 1) acquisition of a strain $i \in X$, 2) clearance of a strain $i \in X, 3$ ) acquisition of a strain $i \notin X$, and 4) clearance of a strain $i \notin X$, respectively. Each term is a product of the proportion of the population in a particular state and the corresponding acquisition or clearance hazards (per capita rates) denoted by $q$. In an example with three strains and $X=\{1\}$, the corresponding terms are 1) $\left.\left.N_{\emptyset} q_{\emptyset \rightarrow\{1\}}, 2\right) N_{\{1\}} q_{\{1\} \rightarrow \emptyset}, 3\right)$ $N_{\{1\}} q_{\{1\} \rightarrow\{1,2\}}+N_{\{1\}} q_{\{1\} \rightarrow\{1,3\}}$, and 4) $N_{\{1,2\}} q_{\{1,2\} \rightarrow\{1\}}+N_{\{1,3\}} q_{\{1,3\} \rightarrow\{1\}}$.

The baseline hazard of clearance for an individual only infected with strain $i$ is denoted by $q_{\{i\} \rightarrow \emptyset}$ and the baseline hazard for a completely susceptible individual to acquire strain $i$ is given by

$$
q_{\emptyset \rightarrow\{i\}}=c \beta_{i} \sum_{X \in \mathcal{S}_{i}} I_{X} .
$$

Here, $c$ is the per capita rate at which hosts make contacts that are relevant for pathogen spread, $\beta_{i}$ is the probability of successfully acquiring strain $i$ given contact with an individual infected with strain $i$, and $\mathcal{S}_{i} \subset \mathcal{S}$ is the subset of states 
containing strain $i$.

Potential interactions between strains are modelled through modification of the baseline acquisition or clearance hazards. The model allows for different structures of interactions, but we only consider the pairwise-symmetric multiplicative structure (see [8] for the alternative structures). To be exact, we assume each strain that is carried to contribute multiplicatively to the hazard of acquiring (or clearing) the incoming (or outgoing) strain. Hence, the hazards of acquisition and clearance in the presence of other strains are

$$
\begin{aligned}
& q_{X \rightarrow X \cup\{i\}}=\prod_{j \in X} k_{i j} q_{\emptyset \rightarrow i}, \\
& q_{X \backslash\{i\} \rightarrow X}=\prod_{j \in X} h_{i j} q_{i \rightarrow \emptyset},
\end{aligned}
$$

where the involved interaction parameters are pairwise-symmetric, i.e. $k_{j i}=k_{i j}$ and $h_{j i}=h_{i j}$ for all $i$ and $j$. Defined as such, $k_{i j}$ and $h_{i j}$ are essentially hazard ratios that act on the baseline hazards. In particular, values equal to one imply no interaction and the deviation from one indicates the strength of interaction. For the mode of acquisition, $k_{i j}<1$ and $k_{i j}>1$ indicate competitive and mutualistic interactions, respectively. Conversely, for the mode of clearance, $h_{i j}<1$ and $h_{i j}>1$ indicate mutualistic and competitive interactions, respectively. Previously, we have shown that when interaction is present in both modes, the overall interaction could be summarised by the ratio of the two interaction parameters $k_{i j} / h_{i j}$, with $k_{i j} / h_{i j}<1$ and $k_{i j} / h_{i j}>1$ indicating competitive and mutualistic interactions, respectively.

Finally, the basic reproduction number of each strain $i$ is given by $R_{0, i}=$ $c \beta_{i} / q_{\{i\} \rightarrow \emptyset}$. In the absence of interactions, only strains having $R_{0, i}>1$ would be expected to survive, with marginal prevalence given by $\sum_{X \in \mathcal{S}_{i}} N_{X}=1-1 / R_{0, i}$. Note, however, that $R_{0, i}>1$ is neither sufficient nor required for survival in the presence of strain interactions, and that rank marginal prevalence need not reflect rank reproduction number. For simplicity, we fixed $c=3$, set $q_{\{i\} \rightarrow \emptyset}=1$ for all strains and drew $\beta_{i}$ randomly to obtain random $R_{0, i}$.

\section{Network construction}

In the interaction networks we consider here, nodes represent pathogen strains and edges the presence of a pairwise interaction between two strains. By connecting each pair of strains with fixed connection probability $\sigma$, independent from other edges, the strain interaction networks resemble Erdös-Rènyi random graphs that become more saturated with higher connection probability [34].

Binary networks only indicating presence or absence of interactions were con- 
verted to weighted networks by attributing strengths of interaction $x_{i j}$, drawn uniformly between $[-\theta, \theta]$, with $\theta>0$ denoting the maximum strength of interaction. In settings where we considered interaction in acquisition only, $\exp \left(x_{i j}\right)$ was used as $k_{i j}$ in the epidemiological model. When we also considered interaction in clearance, aside from $x_{i j}$, which we drew uniformly from $[-\theta, \theta]$, we also drew an additional random number $x_{i j}^{\prime}$ uniformly from $[-\theta / 2, \theta / 2]$. $\exp \left(x_{i j}^{\prime}\right)$ was used as $k_{i j}$ and $\exp \left(x_{i j}^{\prime}-x_{i j}\right)$ as $h_{i j}$.

\section{Sampling cross-sectional datasets}

We sampled random cross-sectional datasets $\left[Y_{1}, Y_{2}, \cdots, Y_{m}\right]^{T}$ from a multivariate binomial distribution that coincides with the steady state of the described epidemiological model, where $m$ is the number of i.i.d. observations, i.e. individuals. For each individual $l, Y_{l}=\left[Y_{1, l}, Y_{2, l}, \cdots, Y_{n, l}\right]$ denotes the sequence of binary random variables [35], with $Y_{i, l}$ denoting the presence of strain $i$ in individual $l$. It follows that $P\left(Y_{i, l}=1 \forall i \in X, Y_{j, l}=0 \forall j \notin X\right)=N_{X}$, where $N_{X}$ is the steady state solution of model (1).

\section{Statistical network inference}

In previous work, we and others have shown that in a two-strain version of model (1), the prevalence of being simultaneously infected with two strains that do not interact, corresponds to the product of marginal prevalence of both strains, provided that host death in age groups susceptible to infection is negligible [21], and there are no unobserved common risk factors [28]. Moreover, the odds ratio of co-occurrence in the two-strain model corresponds to the ratio of interaction parameters in acquisition and clearance $k_{12} / h_{12}$ [28]. When there are more than two strains, however, the correspondence between pairwise association measures and corresponding pairwise composite interaction parameters $k_{i j} / h_{i j}$ might no longer hold, and deviation from independence between two non-interacting strains could be induced by a third strain that interacts with both. The objective here is to capture these (possibly heterogeneous) pairwise interaction parameters from cross-sectional prevalence surveys for more than two strains. For this purpose, we make use of various network inference methods (Appendix A).

The first statistical network inference method we applied are graphical modelling approaches based on log-linear analysis [36]. This technique is generally used to examine the relationship between more than two categorical variables, here denoting presence-absence of each pathogen strain in a host population. To reconstruct networks of strain-specific interactions from co-occurrence data with up to 10 circulating strains, we searched through the subset of decomposable log-linear models, i.e. models whose dependence graph is triangulated [25]. Selection was applied both in a forward (focused on adding edges) and backward 
(focused on removing edges) fashion, using $\mathrm{AIC}$ or $\mathrm{BIC}$ as selection criterion. Strengths of interaction were quantified a posteriori by calculating odds ratios using conditional maximum likelihood estimation from the contingency tables implied by the selected model.

Alternatively, we used the Ising model to reconstruct the simulated networks 24 37]. Here, the probability of a certain pathogen strain being present is modelled as a function of other strains being present at the same time. The Ising model can be shown to be equivalent to certain kinds of log-linear models, but interactions are at most pairwise and the dependence graph does not need to be triangulated. To estimate the presence and strength of interactions, we made use of regularised logistic regressions: iteratively, one variable is regressed onto all others, with a penalty imposed on the regression coefficients to obtain a sparse network representation [38], and with model selection based on the extended BIC [24].

Lastly, we used generalised estimating equations (GEE) to estimate pairwise odds ratios between all strains under consideration. In modelling the associations between multiple strains concomitantly, we used the alternating logistic regression algorithm of the GENMOD procedure in SAS statistical software [26, 32]. The regression framework facilitates the assessment of strain-specific interactions on the basis of Wald tests. To correct for multiple hypothesis testing, we made use of the Benjamini-Hochberg procedure [39].

\section{Host heterogeneity}

In the sensitivity analysis with host heterogeneity, we considered a version of the described multi-strain epidemiological model with two sub-populations that differ in their contact rates relevant for pathogen spread. The proportions of hosts in the high- and low-contact sub-populations are denoted by $p_{1}=20 \%$ and $p_{2}=$ $80 \%$, and the corresponding contact rates by $c_{1}$ and $c_{2}$, respectively. The values of $c_{1}$ and $c_{2}$ were chosen to obtain a coefficient of variation of $80 \%$. Contacts between the two sub-populations follow a classical pattern based on assortativity fraction $\phi$ within host types, that takes the value 0 when mixing between hosts is random and 1 when fully assortative [33]. Here, we fixed $\phi=50 \%$. The contact rate $c_{s r}$ between a 'transmitting' individual from sub-population $s$ and a 'receiving' individual from sub-population $r$ is given by (see Appendix B for derivation)

$$
c_{s r}=\phi c_{s} \delta_{s r}+(1-\phi) \frac{p_{r} c_{r}}{p_{1} c_{1}+p_{2} c_{2}}, \text { for } s, r \in\{1,2\} .
$$

Here, $\delta_{s r}$ is the Kronecker delta (with $\delta_{s r}=1$ if $s=r$ and zero otherwise). Defined accordingly, the baseline hazard for a susceptible individual in sub-population $r$ to 
acquire strain $i$ is given by

$$
q_{r, \emptyset \rightarrow\{i\}}=c_{1 r} \beta_{i} \sum_{X \in \mathcal{S}_{i}} \frac{N_{1, X}}{p_{1}}+c_{2 r} \beta_{i} \sum_{X \in \mathcal{S}_{i}} \frac{N_{2, X}}{p_{2}}, \text { for } r \in\{1,2\} .
$$

Here, $N_{r, X}$ is the proportion of individuals in sub-population $r \in 1,2$ and infection state $X$, with $\sum_{X \in \mathcal{S}} N_{r, X}=p_{r}$. Differential equations for $N_{r, X}$ were defined analogously as in model (1) and the hazard for acquisition in presence of infection with other strains as in equation (3), e.g.

$$
q_{r, X \rightarrow X \cup\{i\}}=\prod_{j \in X} k_{i j} q_{r, \emptyset \rightarrow\{i\}} .
$$

For network reconstruction in the stratified analysis, we performed analyses separately within either sub-population. For the alternative analysis with correction, we included an additional variable $R$ indicating to which sub-population each individual belongs. In the GEE regression framework, we included $\mathrm{R}$ as an explanatory variable (irrespective of strain). In the other network approaches, we added $\mathrm{R}$ to the sequence of variables denoting strain-specific presence, i.e. $Y_{l}^{\prime}=$ $\left[Y_{l}, R\right]$. In effect, the conditional independence structure inferred from $\left[Y_{1}^{\prime}, Y_{2}^{\prime}, \cdots, Y_{m}^{\prime}\right]^{T}$ is augmented by one node representing elevated infection risk, next to nodes representing strains.

\section{References}

[1] K. F. Budden, S. D. Shukla, S. F. Rehman, et al. "Functional effects of the microbiota in chronic respiratory disease". Lancet Respir Med 7.10 (2019), 907-920.

[2] H. M. Rowe, V. A. Meliopoulos, A. Iverson, et al. "Direct interactions with influenza promote bacterial adherence during respiratory infections". Nat Microbiol 4.8 (2019), 1328-1336.

[3] S. Cobey and M. Lipsitch. "Niche and neutral effects of acquired immunity permit coexistence of pneumococcal serotypes". Science 335.6074 (2012), 1376-1380.

[4] N. Egawa, K. Egawa, H. Griffin, et al. "Human papillomaviruses; epithelial tropisms, and the development of neoplasia". Viruses 7.7 (2015), 3863-3890.

[5] A. Lerch, C. Koepfli, N. E. Hofmann, et al. "Longitudinal tracking and quantification of individual Plasmodium falciparum clones in complex infections". Sci Rep 9.1 (2019), 3333.

[6] K. Z. Coyte, J. Schluter, and K. R. Foster. "The ecology of the microbiome: networks, competition, and stability". Science 350.6261 (2015), 663-666.

[7] V. Bucci, B. Tzen, N. Li, et al. "MDSINE: Microbial Dynamical Systems INference Engine for microbiome time-series analyses". Genome Biol 17.1 (2016), 121.

[8] I. Man, K. Auranen, J. Wallinga, et al. "Capturing multiple-type interactions into practical predictors of type replacement following human papillomavirus vaccination". Philos Trans $R$ Soc Lond B Biol Sci 374.1773 (2019), 20180298. 
[9] O. Balmer and M. Tanner. "Prevalence and implications of multiple-strain infections". Lancet Infect Dis 11.11 (2011), 868-878.

[10] E. C. Griffiths, A. B. Pedersen, A. Fenton, et al. "The nature and consequences of coinfection in humans". J Infect 63.3 (2011), 200-206.

[11] S. Alizon, J. C. de Roode, and Y. Michalakis. "Multiple infections and the evolution of virulence". Ecol Lett 16.4 (2013), 556-567.

[12] C. H. van Dorp, M. van Boven, and R. J. De Boer. "Immuno-epidemiological modeling of HIV-1 predicts high heritability of the set-point virus load, while selection for CTL escape dominates virulence evolution". PLoS Comput Biol 10.12 (2014), e1003899.

[13] H. Susi, B. Barrès, P. F. Vale, et al. "Co-infection alters population dynamics of infectious disease". Nat Commun 6 (2015), 5975.

[14] P. S. Wikramaratna, A. Kucharski, S. Gupta, et al. "Five challenges in modelling interacting strain dynamics". Epidemics 10 (2015), 31-34.

[15] S. Howard, C. Donnelly, and M.-S. Chan. "Methods for estimation of associations between multiple species parasite infections". Parasitology 122.2 (2001), 233-251.

[16] G. Regev-Yochay, R. Dagan, M. Raz, et al. "Association between carriage of Streptococcus pneumoniae and Staphylococcus aureus in children". JAMA 292.6 (2004), 716-720.

[17] A. K. Chaturvedi, H. A. Katki, A. Hildesheim, et al. "Human papillomavirus infection with multiple types: pattern of coinfection and risk of cervical disease". J Infect Dis 203.7 (2011), 910-920.

[18] M. Mollers, H. J. Vriend, M. A. van der Sande, et al. "Population- and type-specific clustering of multiple HPV types across diverse risk populations in the Netherlands". Am J Epidemiol 179.10 (2014), 1236-1246.

[19] M. Q. Wilber, P. T. Johnson, and C. J. Briggs. "When can we infer mechanism from parasite aggregation? A constraint-based approach to disease ecology”. Ecology 98.3 (2017), 688702.

[20] S. Alizon, C. L. Murall, E. Saulnier, et al. "Detecting within-host interactions from genotype combination prevalence data". Epidemics 29 (2019), 100349.

[21] F. M. Hamelin, L. J. Allen, V. A. Bokil, et al. "Coinfections by noninteracting pathogens are not independent and require new tests of interaction". PLoS Biol 17.12 (2019), e3000551.

[22] A. Hastings. "Can competition be detected using species co-occurrence data?" Ecology 68.1 (1987), 117-123.

[23] M. Plummer, S. Vaccarella, and S. Franceschi. "Multiple human papillomavirus infections: the exception or the rule? (editorial)". J Infect Dis 203.7 (2011), 891-893.

[24] C. D. Van Borkulo, D. Borsboom, S. Epskamp, et al. "A new method for constructing networks from binary data". Sci Rep 4.1 (2014), 1-10.

[25] S. Højsgaard, D. Edwards, and S. Lauritzen. Graphical models with R. Springer Science \& Business Media, 2012.

[26] V. Carey, S. L. Zeger, and P. Diggle. "Modelling multivariate binary data with alternating logistic regressions". Biometrika 80.3 (1993), 517-526.

[27] E.-M. de Villiers. "Cross-roads in the classification of papillomaviruses". Virology 445.1-2 (2013), 2-10.

[28] I. Man, J. Wallinga, and J. A. Bogaards. "Inferring pathogen type interactions using crosssectional prevalence data: opportunities and pitfalls for predicting type replacement". Epidemiology 29.5 (2018), 666-674.

[29] S. Telfer, R. Birtles, M. Bennett, et al. "Parasite interactions in natural populations: insights from longitudinal data". Parasitology 135.7 (2008), 767-781. 
[30] S. Shrestha, A. A. King, and P. Rohani. "Statistical inference for multi-pathogen systems". PLoS Comput Biol 7.8 (2011), e1002135.

[31] K. P. Burnham and D. R. Anderson. Model selection and multimodel inference: a practical information-theoretic approach. Springer-Verlag, 2004.

[32] S. R. Lipsitz and G. M. Fitzmaurice. "Estimating equations for measures of association between repeated binary responses". Biometrics 52.3 (1996), 903-912.

[33] M. J. Keeling and P. Rohani. Modeling infectious diseases in humans and animals. Princeton University Press, 2008.

[34] M. Newman. Networks. Oxford University Press, 2018.

[35] J. L. Teugels. "Some representations of the multivariate Bernoulli and binomial distributions". J Multivar Anal 32.2 (1990), 256-268.

[36] A. Agresti. Categorical data analysis. Vol. 482. John Wiley \& Sons, 2003.

[37] E. Ising. "Beitrag zur theorie des ferromagnetismus". Zeitschrift für Physik 31 (1925), 253-258.

[38] P. Ravikumar, M. J. Wainwright, J. D. Lafferty, et al. "High-dimensional Ising model selection using I1-regularized logistic regression". Ann Stat 38.3 (2010), 1287-1319.

[39] Y. Benjamini and Y. Hochberg. "Controlling the false discovery rate: a practical and powerful approach to multiple testing". J R Stat Soc Series B Stat Methodol 57.1 (1995), 289-300.

\section{Appendices of Chapter 4}

\section{A. Statistical network inference}

The steady state of model (4.1) represents the expected proportions of the host population that carry different combinations of infectious strains. These can be used to test for interactions between strains. In previous work considering the model with only two strains, we have shown that the prevalence of being simultaneously infected by two non-interacting strains in model (4.1) corresponds to the product of marginal prevalences of the two strains, provided that there are no unobserved common risk factors [28]. Consequently, standard methods such as $\chi^{2}$ tests could be employed to test whether the observed probability of co-occurrence differs from expectation under the null hypothesis of noninteraction, assuming that host death in age groups susceptible to infection is negligible [21]. Note, however, that a third strain, interacting with both non-interacting strains, would also lead to a deviation from this null hypothesis. In the present work, we extend the notion of statistical independence to an arbitrary number of infectious strains. If these do not interact in any way, i.e. if $k_{i j}=1$ and $h_{i j}=1$ for all $i$ and $j$, then their infection dynamics are uncoupled and the expected prevalence of any state $X$, i.e. $N_{X}=\prod_{i \in X} \sum_{Y \in S_{i}} I_{Y}$ at the steady state, where $S_{i}$ is the subset of states containing strain. However, conditional independence in the co-occurrence of multiple strains will be disrupted in the presence of between-strain interactions. The objective here is to capture these (possibly heterogeneous) interactions from cross-sectional prevalence surveys.

Here, we want to investigate if strain-specific interactions in the system as described by equations (4.1)-(4.3) of the main text can be inferred by testing for conditional independence at the steady state. The statement that strain $i$ and $j$ are conditionally independent given strain $k$ means that (co-)infection with strains $i$ and $j$ occurs independently given the presence of strain $k$, i.e.

$$
\frac{\sum_{X \in S_{i}, X \in S_{j}, X \in S_{k}} N_{X}}{\sum_{X \in S_{k}} N_{X}}=\frac{\sum_{X \in S_{i}, X \in S_{k}} N_{X}}{\sum_{X \in S_{k}} N_{X}} \cdot \frac{\sum_{X \in S_{j}, X \in S_{k}} N_{X}}{\sum_{X \in S_{k}} N_{X}} .
$$

If Equation (S4.1) holds, then $\frac{\sum_{X \in S_{i}, X \in S_{j}, X \in S_{k}} N_{X}}{\sum_{X \in S_{j}, X \in S_{k}} N_{X}}=\frac{\sum_{X \in S_{i}, X \in S_{k}} N_{X}}{\sum_{X \in S_{k}} N_{X}}$. This means that, given 
the status of strain $k$, occurrence of strain $i$ is not informed by presence of strain $j$. Note that marginal independence is a special case of conditional independence, where there is no need to condition on any other strain, thus $\sum_{X \in S_{i}, X \in S_{j}} N_{X}=\left(\sum_{X \in S_{i}} N_{X}\right) \cdot\left(\sum_{X \in S_{j}} N_{X}\right)$. By taking logarithms and possibly expanding in $X$ by factorization, Equation (S4.1) takes the form of a log-linear model that can be estimated by Poisson regression [36].

In our example, one could find a parsimonious description of the network of strain-specific interactions by brute-force optimization over all possible log-linear models. However, with 10 circulating strains, the state space already consists of more than a thousand infection states. Therefore, we attempted to uncover the structure of strain-specific interactions from co-occurrence data by searching through the subset of decomposable log-linear models, i.e. models whose dependence graph is triangulated [25]. Selection was applied both in a forward or a backward fashion (i.e. by iteratively growing or shrinking networks, respectively), using AIC or BIC as selection criterion. Computations were performed with the packages gRbase (version 1.8.3), gRain (version 1.3.0), and gRim (version 0.2.0) developed for graphical modelling with $R$ (version 3.4.1) 25]. Strengths of interaction were quantified a posteriori, by calculating odds ratios using conditional maximum likelihood estimation from the contingency tables implied by the selected model.

Alternatively, we made use of a method for constructing networks from binary data, based on the Ising model as known from statistical mechanics [37]. In this model, the conditional probability of a certain pathogen strain being absent or present in the $l$ th-host, represented by the binary node $Y_{i}=y_{i}=\{0,1\}$ given all other nodes $Y_{-i}$ (suppressing $l$ in the notation here for simplicity), is defined by

$$
P\left(Y_{i}=y_{i} \mid Y_{-i}=y_{-i}\right)=Z^{-1} \exp \left(\tau_{i} y_{i}+\sum_{j \neq i} \omega_{i j} y_{i} y_{j}\right) .
$$

Here, $Z=1+\exp \left(\tau_{i}+\sum_{j \neq i} \omega_{i j} y_{j}\right)$ acts as a normalizing constant, and $\omega_{i j}$ are network parameters denoting pairwise interactions between nodes $Y_{i}$ and $Y_{j}$; the higher $\omega_{i j}$ becomes, the more nodes $Y_{i}$ and $Y_{j}$ prefer to be in the same state. Besides, $\tau_{i}$ is a threshold parameter that determines the prevalence of strain $i$ in absence of interactions, and it can be verified that $\tau_{i}=\log \left(R_{0, i}-1\right)$ in our model. By viewing $Y_{i}$ as the response variable and all other variables $Y_{-i}$ as predictors, one may fit a logistic regression function to investigate which nodes are connected to the response variable. The intercept of the regression equation is the threshold of the index node, while the slope of the regression equation is the connection strength with other nodes. To establish which of the variables in the data are connected to a given variable, and which are not, some regularization is required. We used $l 1$-regularised logistic regression [38], commonly known as lasso (least absolute shrinkage and selection operator), with model selection based on the extended BIC [24]. Computations were performed in $\mathrm{R}$ (version 4.0.2) using the packages IsingFit (version 0.3.1) and IsingSampler (version 0.2.1).

Lastly, we also considered generalised estimating equations (GEE) to assess the association between pairs of binary responses, using the alternating logistic regression algorithm of the GENMOD procedure in SAS [26 32]. We have employed this framework before to study clustering patterns of HPV genotypes across risk populations [18]. The alternating logistic regression algorithm seeks to model the logarithm of the odds ratio between strains $i$ and $j$ for the $l$-th individual as $\log \left(\frac{P\left(Y_{i}=1, Y_{j}=1\right) P\left(Y_{i}=0, Y_{j}=0\right)}{P\left(Y_{i}=1, Y_{j}=0\right) P\left(Y_{i}=0, Y_{j}=1\right)}\right)=z_{i j l}^{T} \alpha$. Here, $z_{i j l}$ is obtained from the design matrix, denoting the presence and unicity of pairwise interactions, and $\alpha$ is a vector of regression parameters. The regression framework facilitates the assessment of strain-specific interactions on the basis of Wald tests. To control the rate of type I errors in null hypothesis testing when conducting multiple comparisons, we made use of the Benjamini-Hochberg procedure 39.

\section{B. Host heterogeneity}

The contact rate $c_{s r}$ between a 'transmitting' individual from sub-population $s$ and a 'receiving' individual from sub-population $r$ can be understood as follows. The overall contact rate of individuals from 
a particular sub-population can be decomposed into effective contact rates made with individuals of one's own sub-population $c_{r=s}$ and with individuals from another sub-population $c_{r \neq s}$.

In case of fully assortative mixing $(\phi=1)$, all contacts are made with individuals from one's own sub-population, i.e. if $\phi=1$, then $c_{s} \equiv c_{r=s}$ for $s, r \in\{1,2\}$. In case of random mixing $(\phi=0)$, the probability that a contact belongs to sub-population $r$ is determined by the total share of contacts that are made by individuals from this sub-population. This share is equal to $\frac{p_{r} c_{r}}{p_{1} c_{1}+p_{2} c_{2}}$, i.e. the relative contact rate weighted by the proportion in each sub-population. As mixing patterns do not alter population contact rates, the following relation holds for the effective contact rates made with individuals from either sub-population is $c_{s}=c_{r=s}+c_{r \neq s}=\left(\frac{p_{r=s} c_{r=s}}{p_{1} c_{1}+p_{2} c_{2}}\right) c_{s}+\left(\frac{p_{r \neq s} c_{r \neq s}}{p_{1} c_{1}+p_{2} c_{2}}\right) c_{s}$ for $s, r \in\{1,2\}$.

With an assortment probability $\phi$ the effective contact rate between individuals from distinct subpopulations is scaled down by a factor $1-\phi$, i.e. $c_{r \neq s}=(1-\phi)\left(\frac{p_{r \neq s} c_{r \neq s}}{p_{1} c_{1}+p_{2} c_{2}}\right)$ for $s, r \in\{1,2\}$. Likewise, the effective contact rate between individuals from the same sub-population is given by the weighted average of fully assortative and random mixing $c_{r=s}=\phi \cdot c_{s}+(1-\phi) \cdot\left(\frac{p_{r=s} c_{r=s}}{p_{1} c_{1}+p_{2} c_{2}}\right) c_{s}$ for $s, r \in\{1,2\}$. It follows that both situations are captured by the following generalization: $c_{s r}=$ $c_{s}\left(\phi \cdot \delta_{s r}+(1-\phi) \cdot \frac{p_{r} c_{r}}{p_{1} c_{1}+p_{2} c_{2}}\right)$ for $s, r \in\{1,2\}$. 


\section{Supplementary tables and figures}

\section{Draw presence of interactions}

- Connect each pair of strains, $i$ and $j$, with probability $P\left(x_{i j} \neq 0\right)=\sigma$

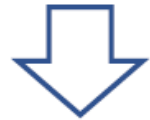

2. Draw strengths of interactions

- Given $x_{i j} \neq 0$, draw $x_{i j}$ with $x_{i j} \sim U[-\log \theta, \log \theta]$

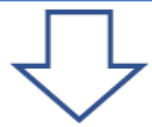

\section{Draw basic reproduction number}

- Draw strain-specific transmission probability $\beta_{i} \sim U\left[\frac{1}{2}, \frac{2}{3}\right]$

- Fix contact rate $c=3$ and clearance rate $q_{\{i\} \rightarrow \emptyset}=1$
Example network with three strains:
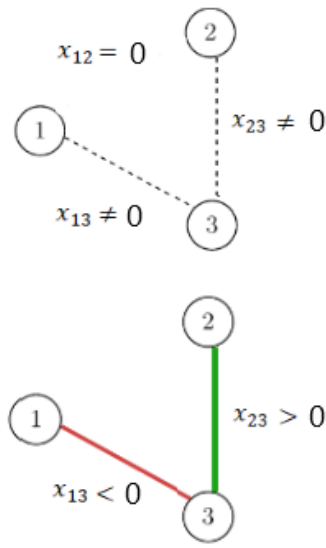

\section{Simulate equilibrium}

- Simulate the epidemiological model until all state-specific prevalence's have stabilized, i.e. $\frac{\mathrm{d} N_{X}}{\mathrm{dt}}=0$

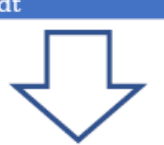

\section{Draw cross-sectional surveys}

- For individual $l=1,2, \ldots, m$ : draw $Y_{l}$ from the steady state $N_{X}$, i.e. $P\left(Y_{i . l}=1 i \in X, Y_{j, l}=0 \forall j \notin X\right)=N_{X}$

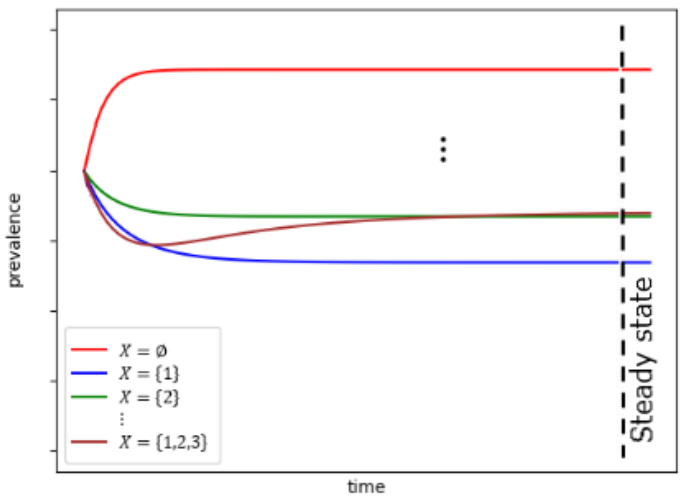

Figure S4.1: Schematic representation of the data generation process. For simplicity, the workflow is illustrated for a system with $n=3$ strains but the same principle was applied in base-case and sensitivity analyses for systems of $n=5$ or $n=10$ strains. 

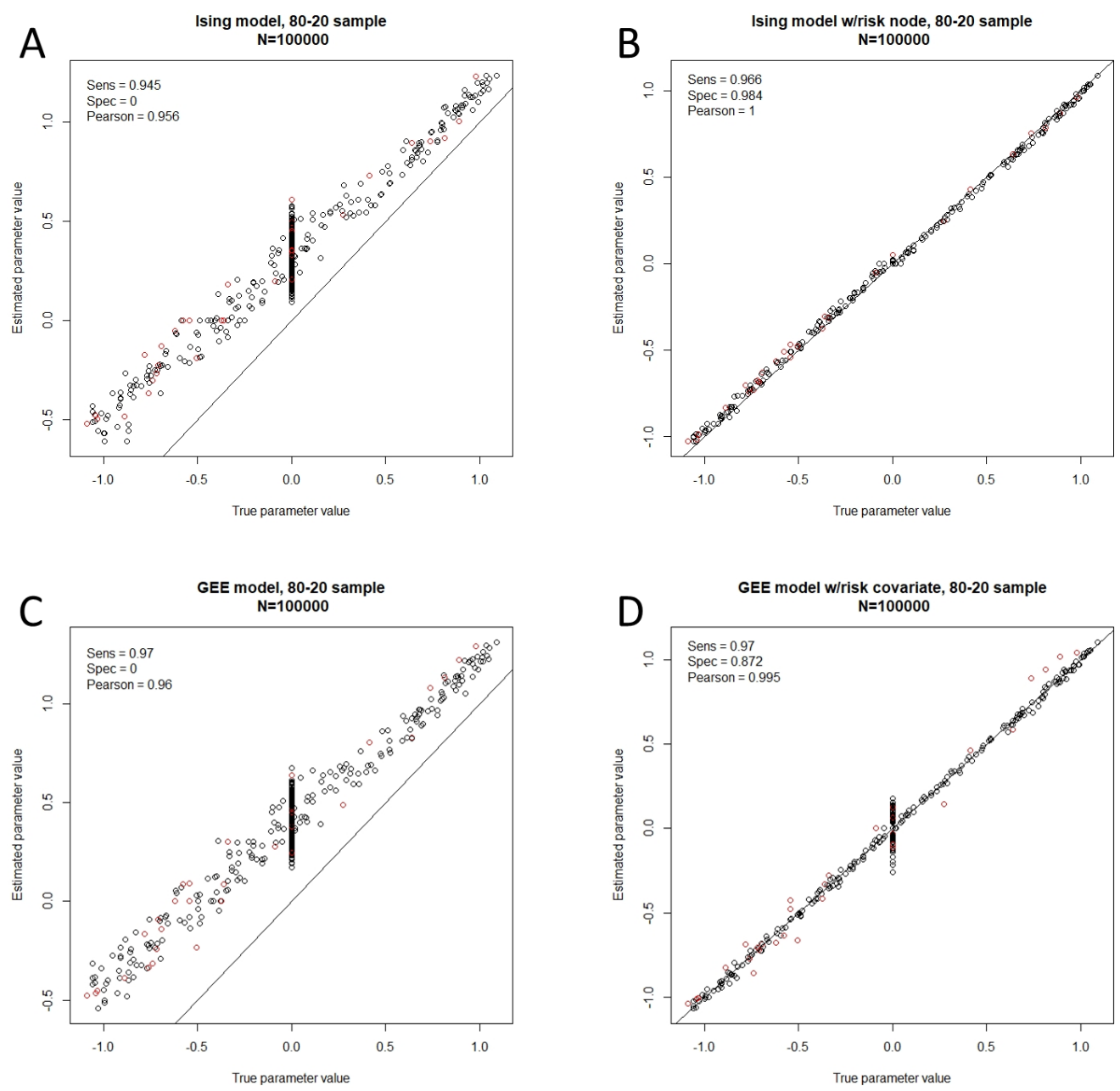

Figure S4.2: Scatter plots of the estimated against the true interaction parameters. Four scatter plots of the estimated against the true interaction parameters under GEE or Ising models, with or without correction for contact rate. 


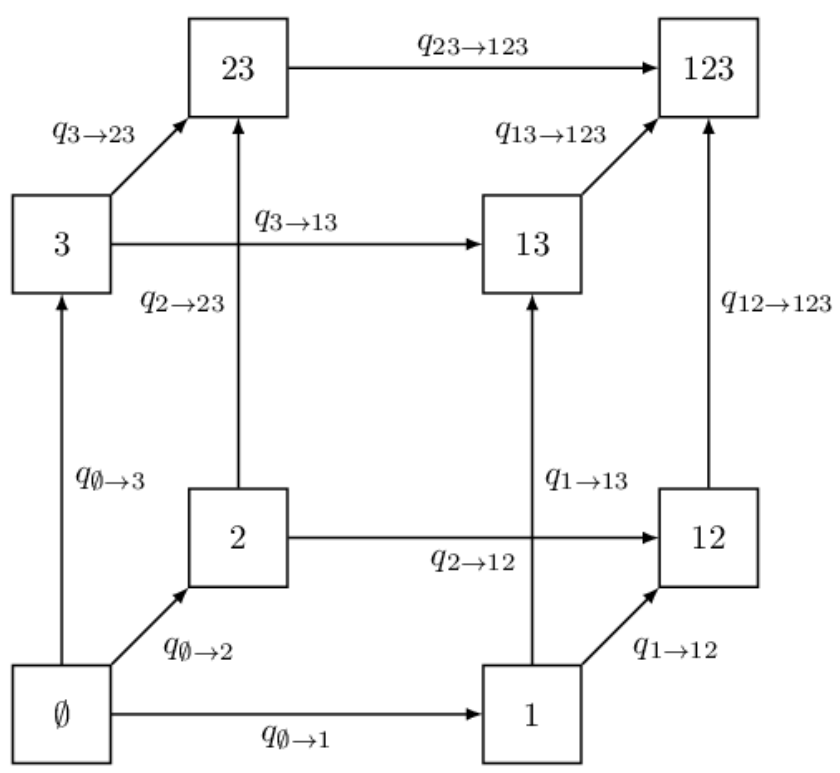

Figure S4.3: Schematic representation of the epidemiological model with $n=3$ strains. The eight $\left(2^{n}\right)$ infection states and transitions pertaining to acquisitions. For convenience, the reverse transitions (i.e. clearances) are not shown, and infectious states are not shown in set notation with brackets, unlike the formulas in the main text. 


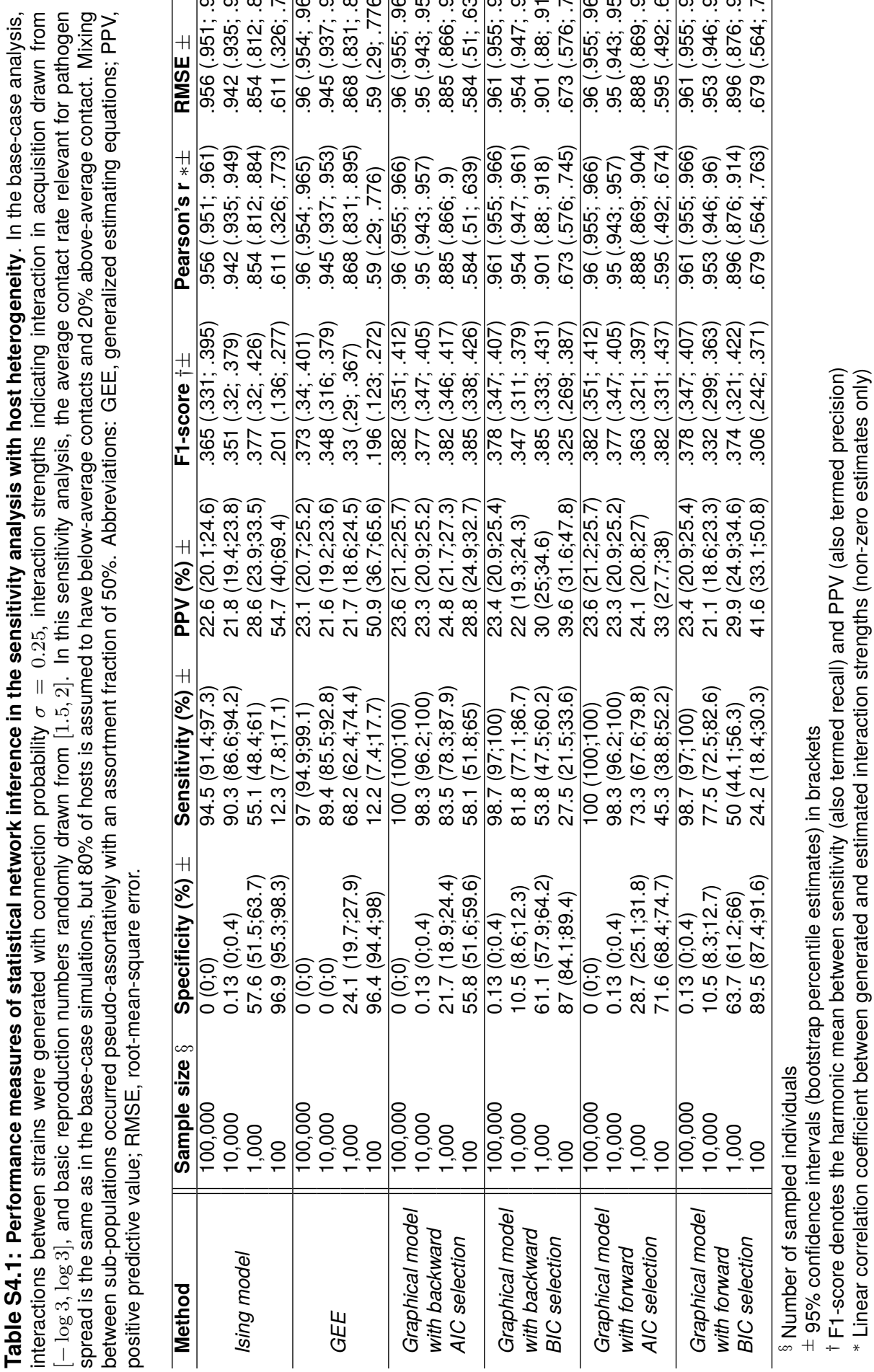




\section{Chapter 5}

\section{Capturing multiple-type interactions into practical pre- dictors of type replacement following human papillo- mavirus vaccination}

This chapter has been published as: I. Man, K. Auranen, J. Wallinga, and J. A. Bogaards. "Capturing multiple-type interactions into practical predictors of type replacement following human papillomavirus vaccination". Philos Trans R Soc Lond B Biol Sci, 374.1773 (2019), 20180298. 


\section{Abstract}

Current HPV vaccines target a subset of the oncogenic human papillomavirus (HPV) types. If HPV types compete during infection, vaccination may trigger replacement by the non-targeted types. Existing approaches to assess the risk of type replacement have focused on detecting competitive interactions between pairs of vaccine and non-vaccine types. However, methods to translate any inferred pairwise interactions into predictors of replacement have been lacking. In this paper, we develop practical predictors of type replacement in a multi-type setting, readily estimable from pre-vaccination longitudinal or cross-sectional cooccurrence data. The predictors we propose for replacement by individual nontargeted types take the form of weighted cross hazard ratios of acquisition versus clearance, or aggregate odds ratios of coinfection with the vaccine types. We elucidate how the hazard-based predictors incorporate potentially heterogeneous direct and indirect type interactions by appropriately weighting type-specific hazards and show when they are equivalent to the odds-based predictors. Additionally, pooling type-specific predictors proves to be useful for predicting increases in the overall non-vaccine-type prevalence. Using simulations, we demonstrate a good performance of the predictors under different interaction structures. Furthermore, we discuss potential applications and limitations of the proposed methodology in predicting type replacement, as compared to existing approaches. 


\section{Introduction}

Predicting the impact of vaccination against a pathogen can be challenging if the pathogen consists of many, potentially interacting (sub)types. When the vaccine targets only a subset of the pathogenic types, it is particularly relevant to evaluate the risk of replacement by the non-targeted types. In this paper, we expand existing methodologies for predicting type replacement for multi-type pathogens, with the focus on the human papillomavirus (HPV).

HPV is one of the most common oncogenic DNA viruses in humans. Persistent infection with HPV can cause cancer in various parts of the body [1]. In particular, twelve to fifteen HPV types are classified as high-risk or probable high-risk due to their association with cervical cancer [2]. Currently, three HPV vaccines are available, covering two, four, and nine HPV types. All three vaccines protect against HPV 16 and 18, two high-risk types that together account for approximately 70\% of all cervical cancers in unvaccinated populations. HPV 31, 33, and 45, accounting for an additional $10-15 \%$ of cases [3], are among the cross-protective types of the bivalent and quadrivalent vaccines and are included in the nonavalent vaccine [4].

In countries where HPV vaccination has been implemented with high coverage, circulation of both the vaccine and cross-protective HPV types has decreased considerably [5, 6]. However, concerns have been raised that the ecological niche vacated by the targeted types could be taken over by the non-targeted high-risk HPV types [7-9]. Thus far, post-implementation surveillance data have been reassuring, as only sporadic increases in the prevalence of infection with some nonvaccine types have been observed without a clear signal of type replacement 4 10-12]. While waiting for the long-term impact of HPV vaccination to become apparent, evaluation of the potential for type replacement remains crucial.

Whether HPV vaccination will trigger type replacement depends on the existence and strength of competitive interactions between HPV types. HPV types may compete during coinfection, by either diminishing each other's opportunities to establish a productive infection or by promoting viral clearance (e.g. through activation of antigen-presenting cells) [13-17]. Due to such competitive mechanisms, the hazard of acquiring (or clearing) a given HPV type may be reduced (or increased, respectively) by infection with other types. In epidemiological terms, different type interactions can be conveniently quantified in terms of appropriately defined hazard ratios [18-20].

We have previously shown, in a simple model of one vaccine and one nonvaccine type, that the cross hazard ratio of acquisition versus clearance can be used to predict type replacement, provided that the two types interact symmetrically and there is no long-lasting cross-immunity [21]. The latter assumption seems plausible for HPV, as even the existence of natural homologous immunity 
is still debated [22, 23]. Given Susceptible-Infected-Susceptible (SIS) transmission dynamics, the appropriate cross hazard ratio is, moreover, equivalent to an odds ratio of coinfection, and thus estimable from cross-sectional prevalence data [21]. Here, the odds ratio is defined as the odds of infection with one type in presence versus absence of coinfection with the other type.

With more than two interacting types, not only does the prediction of replacement require inference of interactions between multiple types but also an adequate way of combining them. To predict how vaccination will affect the prevalence of a given non-vaccine type, one needs to take account of its direct interactions with the vaccine types as well as any indirect interactions via other non-vaccine types. Meaningful prediction should also incorporate possible heterogeneities in strength or direction (competition versus synergy) of type interactions.

Previous studies evaluating the potential of HPV type replacement have focused on inferring interactions between pairs of vaccine and non-vaccine types [7. 24-27]. In these studies, for each vaccine type, pairwise odds ratios have been compared to pooled odds ratios (pooled across the non-vaccine types) to identify likely candidates for type replacement. With this approach, however, each non-vaccine type is evaluated multiple times according to its interactions with different vaccine types, while the occurrence of type replacement is determined by all these interactions jointly. In addition, the pooled odds ratio, which has been interpreted as the tendency of the vaccine type in question to be involved in coinfection, lacks a clear interpretation for the prediction of type replacement.

In this paper, we consider the prediction of replacement following vaccination in a setting with an arbitrary number of interacting vaccine and non-vaccine types. We propose predictors of type-specific replacement, i.e. increase in the prevalence of individual non-vaccine types, and pooled predictors of increase in the overall non-vaccine-type prevalence. The predictors are initially defined in terms of steady-state hazards of type-specific acquisition and clearance. Using a SIS model, we explain how the predictors relate to the underlying mode of type interactions and show under which interaction structures these hazard-based predictors can be estimated as odds ratios from cross-sectional prevalence data. Finally, we evaluate the performance of the proposed predictors by means of numerical simulations.

\section{Material and methods}

\section{Prediction framework}

We derive predictors of type replacement for a pathogen consisting of many potentially interacting types. The prediction method applies to any pathogen for which naturally acquired immunity is short-lived so that the transmission dynamics of 
each type can be approximated by an SIS model. The predictors are constructed in terms of the following data collected at the pre-vaccination steady state:

- prevalence of each (co)infection state, estimable from cross-sectional data;

- type-specific hazards (per capita rates) of acquisition and clearance, estimable from longitudinal data.

Figure 5.1A shows the eight (co)infection states and the respective transitions for a pathogen with three types.

Type interaction is assumed to operate through current (co)infection, with one or multiple types modifying acquisition hazards of other types, or modifying clearance hazards of coinfecting types. Interactions between different types are allowed to be either competitive or synergistic and to vary in strength. Furthermore, we focus on predicting replacement by non-vaccine types that are not crossprotected by the vaccine, as these types are the most salient for evaluating the potential for replacement.

\section{Type-specific and overall replacement}

We consider replacement, here defined as an increase in the prevalence of nonvaccine-type infection once the vaccine types are eliminated in the post-vaccination steady state, at two levels:

- type-specific replacement, defined as an increase in the prevalence of a given non-vaccine type $i$. This occurs when $\sum_{i \in X} I_{X}^{\prime} / \sum_{i \in X} I_{X}>1$, where $I_{X}^{\prime}$ and $I_{X}$ denote the post- and pre-vaccination steady-state prevalence of infection state $X$, respectively. The sums here are taken over all states containing type $i$, e.g. states $\{3,13,23,123\}$ in a three-type system when considering $i=3$;

- overall replacement, defined as an increase in the overall non-vaccine-type prevalence. This occurs when $\sum_{|X \cap N V T|>0} I_{X}^{\prime} / \sum_{|X \cap N V T|>0} I_{X}>1$, where $N V T$ is the set of all non-vaccine types, and $|\cdot|$ denotes the number of types in a given set. The sums are taken over all states containing at least one non-vaccine type, e.g. states $\{2,3,23,12,13,123\}$ in a three-type system when considering $N V T=\{2,3\}$.

\section{Predictors in a two-type setting}

Previously, we have shown that the following pairwise odds ratio is an exact predictor of type replacement in a simple SIS model of one vaccine type (type 1) and 

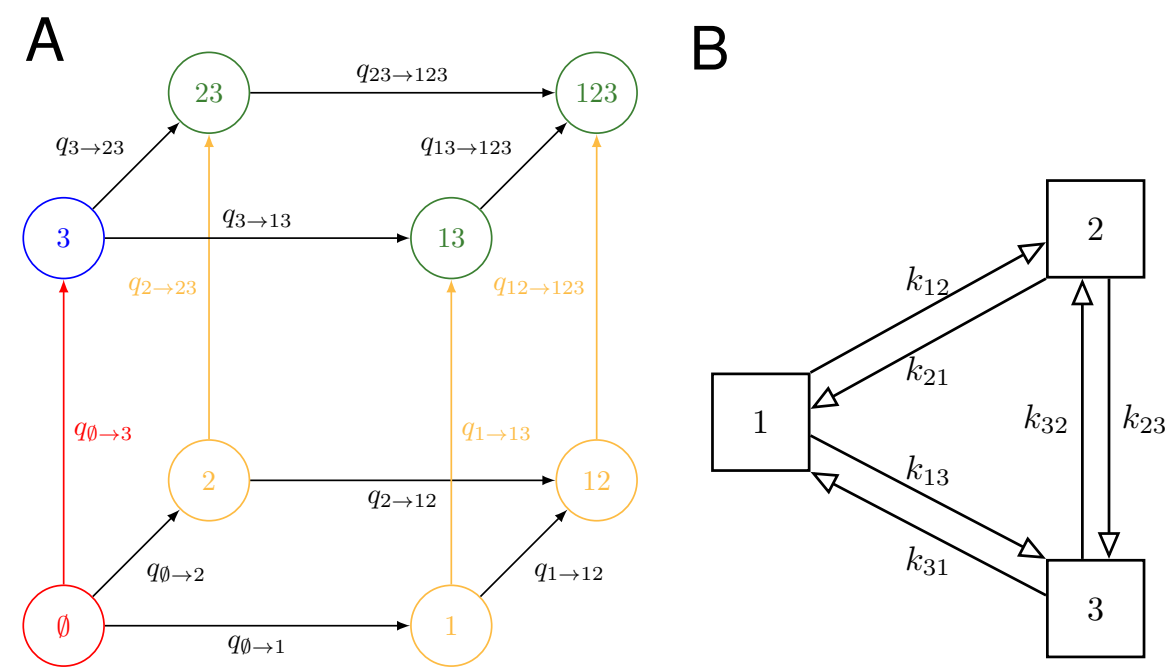

Figure 5.1: A) The eight infection states and transitions pertaining to acquisitions in a threetype system. For convenience, the reverse transitions (i.e. clearances) are not shown. The colours indicate the aggregate states for $V T=\{1,2\}, i=3$ and the corresponding type-3 acquisitions; $\mathcal{A}_{V T}$ in yellow, $\mathcal{A}_{V T, i}$ in green, $\mathcal{A}_{i}$ in blue, $\mathcal{A}_{0}$ in red. B) Graphical representation of the interaction parameters under the symmetric multiplicative structure or pairwise-asymmetric multiplicative structure in a three-type system. Under the pairwisesymmetric multiplicative structure, pairs of reverse interaction parameters would moreover be identical (i.e. $k_{i j}=k_{j i}$ ).

one non-vaccine type (type 2), provided the two types interact symmetrically [21]:

$$
O R_{1,2}=\left(\frac{I_{12}}{I_{1}}\right) /\left(\frac{I_{2}}{I_{\emptyset}}\right) .
$$

This pairwise odds ratio compares the odds of non-vaccine-type infection between those infected and uninfected with the vaccine type.

Whenever the pre-vaccination steady-state value of $O R_{1,2}$ is less than one, replacement will occur. This correspondence follows from $O R_{1,2}$ being equivalent to the following pairwise cross hazard ratio:

$$
H R_{1,2}=\left(\frac{q_{1 \rightarrow 12}}{q_{\emptyset \rightarrow 2}}\right) /\left(\frac{q_{12 \rightarrow 1}}{q_{2 \rightarrow \emptyset}}\right),
$$

where $q_{X \rightarrow Y}$ denotes the transition hazard from state $X$ to $Y$. The numerator (denominator) of expression Equation (5.2) is the hazard ratio of acquiring (clearing) the non-vaccine type in those infected versus those uninfected with the vaccine type and thus quantifies interaction in acquisition (clearance). In other words, the numerator (denominator) quantifies to what extent vaccine-type infection accel- 
erates or decelerates acquisition (clearance) of non-vaccine-type infection. As a whole, the ratio captures the joint effects of interactions both in acquisition and clearance on the occurrence of non-vaccine-type infection.

Of note, the above pairwise odds ratio Equation (5.1) has been used for inferring interactions between HPV genotypes [7. 25-27], whereas the pairwise cross hazard ratio Equation (5.2) has been used for describing competition between Streptococcus pneumoniae serotypes [28].

\section{Predictors in a multi-type setting}

Type-specific cross hazard ratio as a predictor of type-specific replacement

We now generalise the pairwise cross hazard ratio Equation (5.2) to a setting with an arbitrary number of interacting vaccine and non-vaccine types. To predict replacement by a given non-vaccine type $i$, we first aggregate the infection states into the four disjoint collections of states given by Table 5.1 (see Figure 5.1A). Based on the transitions between these four aggregate states, we propose the following generalization of the four hazards in the pairwise cross hazard ratio Equation (5.2)

$$
H R_{V T, i}=\left(\frac{\sum_{X \in \mathcal{A}_{V T}} I_{X \mid \mathcal{A}_{V T}} q_{X \rightarrow X \cup\{i\}}}{\sum_{X \in \mathcal{A}_{0}} I_{X \mid \mathcal{A}_{0}} q_{X \rightarrow X \cup\{i\}}}\right) /\left(\frac{\sum_{X \in \mathcal{A}_{V T, i}} I_{X \mid \mathcal{A}_{V T, i}} q_{X \rightarrow X \backslash\{i\}}}{\sum_{X \in \mathcal{A}_{i}} I_{X \mid \mathcal{A}_{i}} q_{X \rightarrow X \backslash\{i\}}}\right),
$$

where $I_{X \mid \mathcal{A}}=I_{X} / \sum_{Y \in \mathcal{A}} I_{Y}$ denotes the relative prevalence of state $X$ conditioned within an aggregate state $\mathcal{A}$. Each term in expression Equation (5.3) is a weighted average of hazards as it aggregates hazards of acquiring or clearing type $i$ with weights given by the conditional steady-state prevalence of the states from which the transitions occur. In effect, this weighting adjusts for the time each individual spends being at risk for the respective transitions [29]. Similar to the pairwise cross hazard ratio Equation (5.2) the numerator and denominator of the type-specific cross hazard ratio Equation (5.3) are hazard ratios of acquiring and clearing type $i$ in those infected with at least one vaccine type $(|X \cap V T|>0)$ versus those uninfected with any of the vaccine types $(|X \cap V T|=0)$, respectively. In short, the type-specific cross hazard ratio Equation (5.3) combines interactions by the vaccine types on the non-vaccine type of interest.

An example with multiple vaccine types -Assume that $V T=\{1,2\}, N V T=$ $\{3\}$, and there is interaction only in acquisition (Figure 5.1 $\mathrm{A}$ ), and consider the type-specific cross hazard ratio Equation (5.3) as a predictor for replacement by type 3. As the denominator of expression Equation (5.3) now equals one, the 
Table 5.1: Four disjoint collections of infection states constructed for the definition of the type-specific cross hazard ratio Equation (5.3). Here, $V T$ denotes the set of vaccine types.

\begin{tabular}{|c|c|}
\hline $\begin{array}{c}\mathcal{A}_{V T}=\{X:|X \cap V T|>0, i \notin X\} \\
\text { states with vaccine type(s), without type } i\end{array}$ & $\begin{array}{l}\mathcal{A}_{V T, i}=\{X:|X \cap V T|>0, i \in X\} \\
\text { states with vaccine type(s), with type } i\end{array}$ \\
\hline $\begin{array}{c}\mathcal{A}_{0}=\{X:|X \cap V T|=0, i \notin X\} \\
\text { states without vaccine type(s), without type } i\end{array}$ & $\begin{array}{c}\qquad \mathcal{A}_{i}=\{X:|X \cap V T|=0, i \in X\} \\
\text { states without vaccine type(s), with type } i\end{array}$ \\
\hline
\end{tabular}

predictor reduces to

$$
H R_{12,3}=\frac{I_{1 \mid\{1,2,12\}} q_{1 \rightarrow 13}+I_{2 \mid\{1,2,12\}} q_{2 \rightarrow 23}+I_{12 \mid\{1,2,12\}} q_{12 \rightarrow 123}}{q_{\emptyset \rightarrow 3}},
$$

where $I_{1 \mid\{1,2,12\}}=I_{1} / C, I_{2 \mid\{1,2,12\}}=I_{2} / C, I_{12 \mid\{1,2,12\}}=I_{12} / C$, and $C=I_{1}+I_{2}+$ $I_{12}$.

\section{Type-specific odds ratio as a predictor of type-specific replacement}

The type-specific cross hazard ratio $H R_{V T, i}$ as given by Equation (5.3) requires estimation of type-specific acquisition and clearance hazards from longitudinal data. However, collecting such data may be cumbersome and expensive due to repeated observations of the infection states in the same study subjects. It would thus be advantageous if the cross hazard ratio $H R_{V T, i}$ could be approximated using steady-state cross-sectional (i.e. prevalence) data, in a similar way as in the two-type setting using the pairwise odds ratio Equation (5.1)

In a setting with more than two types, the pairwise odds ratio Equation (5.1) can be generalised to the following type-specific odds ratio:

$$
O R_{V T, i}=\left(\frac{\sum_{X \in \mathcal{A}_{V T, i}} I_{X}}{\sum_{X \in \mathcal{A}_{V T}} I_{X}}\right) /\left(\frac{\sum_{X \in \mathcal{A}_{i}} I_{X}}{\sum_{X \in \mathcal{A}_{0}} I_{X}}\right),
$$

which is the odds of infection with non-vaccine type $i$ in presence versus absence of coinfection with any of the vaccine types. The approximation of $H R_{V T, i}$ by $O R_{V T, i}$ is exact given detailed balance, i.e. when $I_{X} q_{X \rightarrow Y}=I_{Y} q_{Y \rightarrow X}$ for any pair of states $X, Y$ (see Appendix A for the proof). In reality, whether this property holds depends on the underlying structure of type interactions.

Example continued - In the previous example where $V T=\{1,2\}, N V T=\{3\}$ (Figure 5.1A), the type-specific odds ratio Equation (5.5) becomes

$$
O R_{12,3}=\left(\frac{I_{13}+I_{23}+I_{123}}{I_{1}+I_{2}+I_{12}}\right) /\left(\frac{I_{3}}{I_{\emptyset}}\right) .
$$


Overall odds and cross hazard ratios as predictors of overall type replacement

Pooling across pairwise odds ratios has been used to summarise interactions of all non-vaccine types with each vaccine type separately [7, 25]. However, this approach lacks a clear interpretation for predicting type replacement. Here, we propose the following overall odds ratio for predicting overall replacement:

$$
O R=\prod_{i \in N V T} O R_{V T, i}
$$

The use of a pooled odds ratio for the prediction can be justified by considering the ratio of the odds of overall non-vaccine-type infection in the pre- versus postvaccination steady states. This odds ratio is essentially the true impact of vaccination with values less than one characterizing overall replacement. Assuming mutual independence between all non-vaccine types, this true odds ratio of overall infection can be approximated by the product of true odds ratios of type-specific infections (see Appendix B for the derivation). As each true type-specific odds ratio can be predicted by the corresponding $O R_{V T, i}$, we envision their product Equation (5.7) to be a predictor for overall replacement.

Owing to the relation between $H R_{V T, i}$ and $O R_{V T, i}$, we also propose the following overall cross hazard ratio as a predictor for overall replacement:

$$
H R=\prod_{i \in N V T} H R_{V T, i}
$$

\section{Simulated structures of type interactions}

Thus far, we have made no assumptions about the structure of type interactions, i.e. no constraints on $k_{X i}$ and $h_{X i}$ in defining $q_{X \rightarrow X \cup\{i\}}=k_{X i} \cdot q_{\emptyset \rightarrow i}$ and $q_{X \cup\{i\} \rightarrow X}=$ $h_{X i} \cdot q_{\emptyset \rightarrow i}$. To investigate the performance of the proposed predictors and their robustness against different interaction structures, we considered the following three alternative structures in simulations (see Appendix $C$ for the exact descriptions).

The first interaction structure is pairwise-symmetric and multiplicative so that each (co)infecting type contributes multiplicatively to the acquisition hazard of an incoming type, i.e. $q_{X \rightarrow X \cup\{i\}}=\left(\prod_{j \in X} k_{j i}\right) \cdot q_{\emptyset \rightarrow i}$, or to the clearance hazard of a coinfecting type, i.e. $q_{X \cup\{i\} \rightarrow X}=\left(\prod_{j \in X} h_{j i}\right) \cdot q_{i \rightarrow \emptyset}$. Here $k_{j i}$ and $h_{j i}$ describe how (co)infection with type $j$ modifies acquisition and clearance of type $i$, respectively. Values of $k_{j i}$ and $1 / h_{j i}$ equal to, less, and larger than one correspond to independent, competitive, and synergistic interactions, respectively. In order words, competition decreases the acquisition rate, whereas synergy decreases the clearance rate. Furthermore, pairs of interaction parameters are assumed to be identical, i.e. $k_{i j}=k_{j i}$ and $h_{i j}=h_{j i}$, resulting in a symmetric interaction matrix. With $n$ types, the numbers of parameters governing the interaction in acquisition 
and clearance are both reduced from $n\left(2^{n-1}-1\right)$ to $n(n-1) / 2$ (Figure 5.1 3$)$. This pairwise-symmetric multiplicative structure preserves the detailed balance property (see Appendix D for the proof), so that $H R_{V T, i}$ and $O R_{V T, i}$ are equivalent.

Departure from the above structure may disrupt this equivalence of $H R_{V T, i}$ and $O R_{V T, i}$. Two alternative structures are the pairwise-asymmetric multiplicative and the groupwise-symmetric multiplicative structure. The pairwise-asymmetric multiplicative structure relaxes the symmetry constraint $k_{j i}=k_{i j}$. The groupwisesymmetric multiplicative structure assumes the multiplicativity to act on groups of types instead of individual types. For example, in a four-type system with groups $A=\{1,2\}, B=\{3,4\}$, and interaction parameters $k_{A}, k_{B}$ (within groups), $k_{A B}=$ $k_{B A}$ (between groups), the hazards of acquiring type 1 from state 23 and 234 are both $k_{A} k_{B A} q_{\emptyset \rightarrow 1}$.

\section{Performance analysis}

To evaluate the performance of the proposed predictors, we simulated the effect of vaccination using a deterministic SIS transmission model (see Appendix C for details of the model). We investigated how the performance of the predictors depends on the numbers of vaccine and non-vaccine types and their interaction structure. For each setting, different sets of model parameters, including interaction parameters and type-specific transmissibility, were randomly generated.

The interaction parameters of the two symmetric structures were uniformly generated on a log scale in the interval $(1 / 3,3)$, ranging from competitive to synergistic interactions. To examine the effect of increasing asymmetry under the pairwise-asymmetric structure, asymmetric interaction parameters $\left(k_{i j}^{\prime}, k_{j i}^{\prime}\right)$ were obtained by either perturbing the generated parameters of the pairwise-symmetric structure $\left(k_{i j}\right)$, or by generating new $k_{i j}^{\prime}, k_{j i}^{\prime}$ independently of each other. Perturbation of the pairwise-symmetric parameters was done by adding deviations on a log scale, i.e. $\log \left(k_{i j}^{\prime}\right)=\log \left(k_{i j}\right)+\epsilon / 2$ and $\log \left(k_{j i}^{\prime}\right)=\log \left(k_{i j}\right)-\epsilon / 2$. In effect, $e^{\epsilon}$ is the ratio between a pair of reverse interaction parameters, $k_{i j}^{\prime} / k_{j i}^{\prime}$ with increasing $\epsilon$ inducing more asymmetry. When generating $k_{i j}^{\prime}, k_{j i}^{\prime}$ independently, there was no constraint on their ratio.

For each parameter set, prediction of replacement was made at the simulated pre-vaccination steady state and compared to the 'true' outcome (replacement/no replacement) at the post-vaccination steady state. For each setting, the performance of each predictor was defined as the proportion of correct predictions among all generated parameter sets (see Appendix E for the exact simulation procedure). 


\section{Simulation results}

\section{Performance under the pairwise-symmetric multiplicative structure}

As the pairwise-symmetric multiplicative structure obeys detailed balance, the hazard-based and odds-based predictors are equivalent $\left(H R_{V T, i}=O R_{V T, i}\right.$ and $H R=O R$ ) and indeed performed identically (row 1 of Figure 5.2).

With one non-vaccine type, the predictors $\left(H R_{V T, i}=O R_{V T, i}=H R=O R\right)$ were always correct, even when some vaccine types would interact synergistically while others would compete with the non-vaccine type. Thus, the predictors correctly captured the balance between the opposing forces of competition and synergy.

With multiple non-vaccine types, prediction of both type-specific and overall replacement became more difficult due to interactions between the non-vaccine types. For example, in a system with $V T=\{1\}, N V T=\{2,3\}$, if $k_{13}=1, k_{12}>1$ and $k_{23}<1$ (Figure 5.13), vaccination indirectly triggered replacement by type 3 by decreasing type-2 infection and thus increasing type-3 infection. Rewriting Equation (5.3) shows that this indirect effect is indicated by the second factor in

$$
H R_{1,3}=k_{13} \cdot\left[\frac{\left(I_{\emptyset}+I_{2} k_{12} k_{23}\right) \cdot\left(I_{\emptyset}+I_{2}\right)}{\left(I_{\emptyset}+I_{2} k_{12}\right) \cdot\left(I_{\emptyset}+I_{2} k_{23}\right)}\right] .
$$

In particular, the expression in the brackets is less than one when either $k_{12}$ or $k_{23}$ (but not both) is less than one (see Appendix $\mathrm{F}$ for the derivation of Equation (5.9)). However, if the direct and indirect effects acted in opposite directions, the predictors did not always adequately capture their combined effect (see Appendix $G$ for an example). Indeed, with multiple non-vaccine types, the performance decreased as the number of non-vaccine types increased (follow each subfigure in Figure 5.2 across the $\searrow$ diagonals). Nonetheless, the performance of all predictors remained well above $94 \%$. In addition, when the overall predictor failed with a value close to one, the overall prevalence of non-vaccine-type infection usually changed only modestly (see Appendix $G$ for an example).

\section{Performance under alternative interaction structures}

Under the alternative interaction structures, the hazard-based and odds-based predictors were no longer equivalent but still performed comparably, except when the asymmetry was strong (Figure 5.2. With one non-vaccine type, the hazardbased predictions remained almost perfect, unlike the odds-based ones. Under the groupwise-symmetric structure, the predictive performance decreased more rapidly as the number of non-vaccine types increased, as compared to the pairwisesymmetric structure. However, it was still above $80 \%$ in simulations with up to 
seven types (row 2 of Figure 5.2).

Under the pairwise-asymmetric structure, the performance of both sets of predictors decreased with increasing asymmetry (rows 3 to 5 of Figure 5.2). Nevertheless, the performance of the hazard-based predictors remained above $85 \%$ even when pairs of $k_{i j}$ and $k_{j i}$ were generated independently, inducing strong asymmetry. The decreasing performance was more vivid for the odds-based predictors, which went down to $70 \%$ in the corresponding settings. The superiority of the hazard-based predictors can be illustrated in the following example of two types. Suppose that the vaccine type competes with the non-vaccine type $\left(k_{12}<\right.$ 1 ) while the reverse interaction is synergistic $\left(k_{21}>1\right)$. Then $H R_{1,2}=k_{12}<1$ predicts replacement correctly, whereas the symmetrically defined $O R_{1,2}$ may not because it averages over $k_{12}$ and $k_{21}$.

The above results were based on simulations in which interactions occurred only in acquisition. Additional simulations that allowed interactions in both acquisition and clearance showed almost identical performance of both the hazard-based and odds-based predictors (compare Figure 5.2 to Figure S5.5 in Appendix H).

\section{Discussion}

This work aims to develop a methodology for predicting type replacement for pathogens with many, potentially interacting types using pre-vaccination epidemiological data. We have proposed a predictor of replacement by individual nonvaccine types (i.e. increase in type-specific prevalence) and another predictor of increase in the overall non-vaccine-type prevalence. Both predictors are initially defined in terms of hazards of acquiring and clearing the non-vaccine types and thus, in principle, require estimation of longitudinal data. In addition, we have derived alternative predictors that can be readily estimated from cross-sectional prevalence data as odds ratios. All proposed predictors demonstrated satisfactory performance (mostly above $85 \%$ ) across a range of simulated structures of type interactions except when the interactions were strongly asymmetric.

The predictor of replacement by a given non-vaccine type is constructed as a weighted cross hazard ratio of acquiring versus clearing infection with the nonvaccine type in question, comparing those infected and uninfected with any of the vaccine types. The odds-based alternative is defined as the odds of infection with that non-vaccine type in the presence versus absence of infection with any of the vaccine types.

We note the similarity between estimators for type interaction and vaccine efficacy of multi-valent vaccines, as both are defined in terms of cross ratios of weighted hazards in a multi-type setting. In both cases, it proves useful to weigh hazards with the steady-state prevalence, conditioned on appropriate risk sets. In 


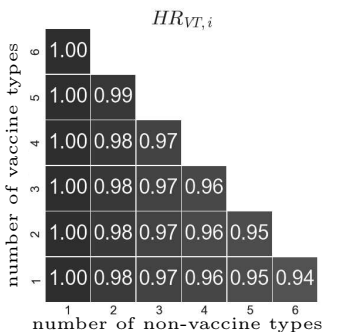

Groupwise-symmetric:
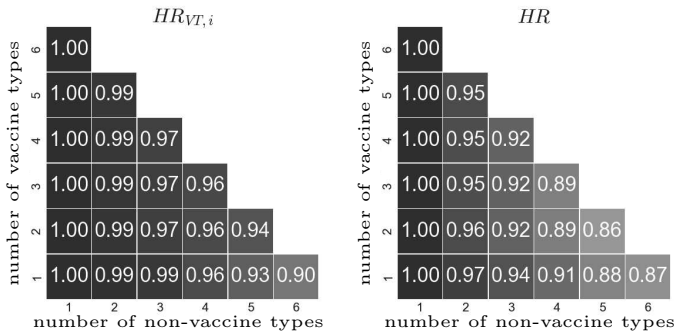

Mildly pairwise-asymmetric $(\epsilon=0.2)$ :
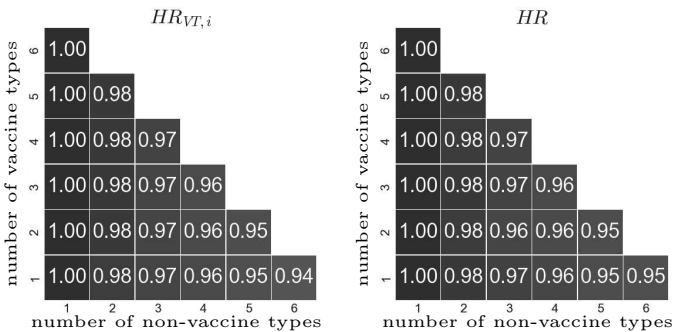

Moderately pairwise-asymmetric $(\epsilon=0.5)$ :
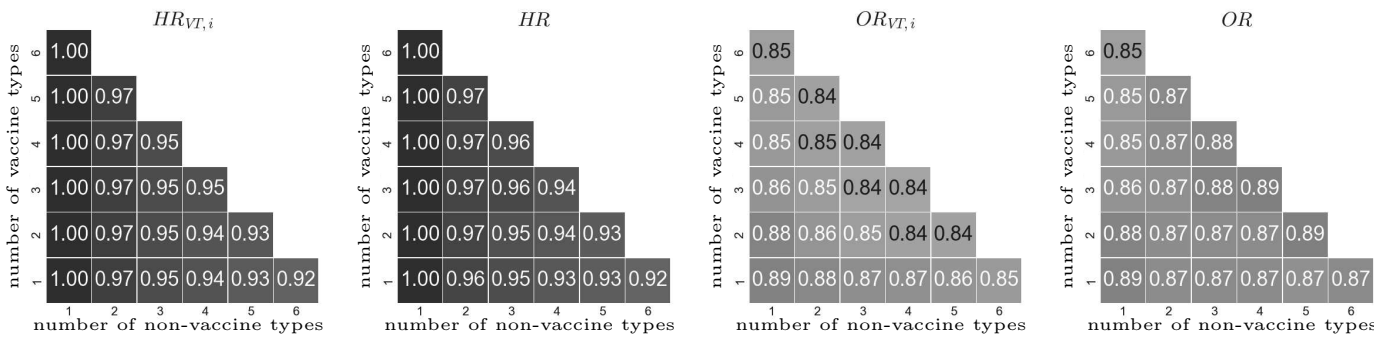

Strongly pairwise-asymmetric:
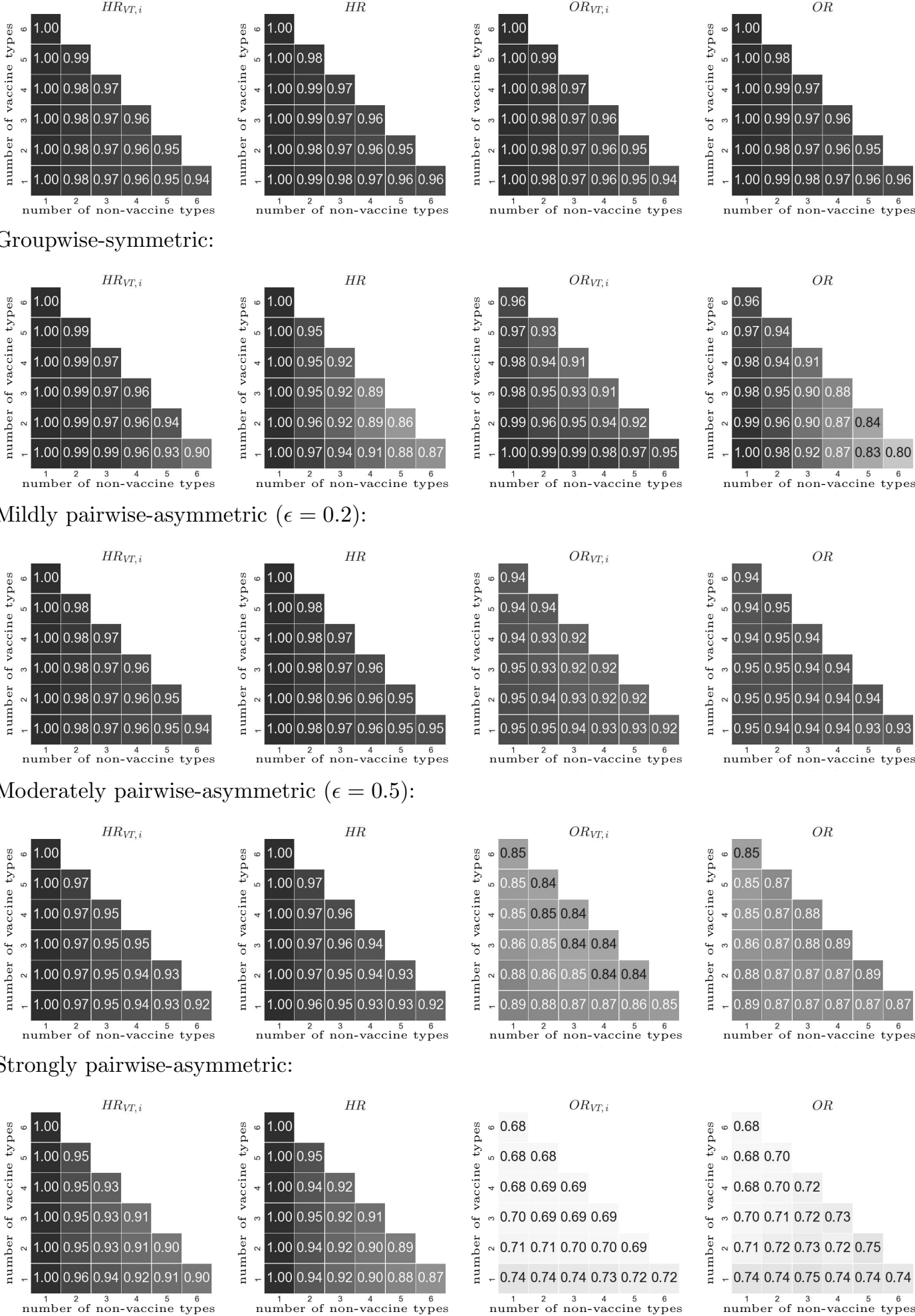

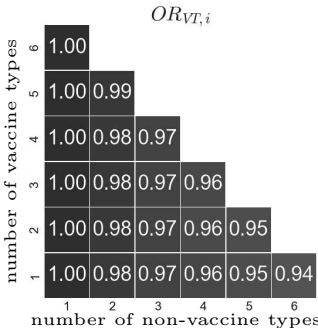


defining estimators for vaccine efficacy against one or more vaccine types, the risk sets are those from which acquisitions of the vaccine types may occur [29]. Likewise, in the current paper, the risk sets are those from which acquisitions (or clearances) of the non-vaccine type may occur (see the type-specific cross hazard ratio Equation (5.3).

The predictors of overall replacement take form as products of either the hazardbased or the odds-based type-specific predictors. In effect, the overall predictors are obtained by pooling the type-specific predictors on a logarithmic scale. In various studies that have used pairwise odds ratios to evaluate HPV type interactions, pooling has been performed across the pool of non-vaccine types for each vaccine type separately [7, 25, 26]. These pooled odds ratios have been interpreted as the affinity of a given vaccine type to be involved in coinfection with any of the nonvaccine types, but their relevance for predicting type replacement has remained elusive. Our results substantiate the predictive value of pooled odds ratios regarding overall replacement but suggest that pooling is better performed on the entire set of vaccine types, instead of for each vaccine type separately. Furthermore, the pairwise odds ratios have been compared to the pooled ones to identify likely candidates for type replacement [7, 25, 26]. Accordingly, the potential for replacement by a given non-vaccine type has been assessed separately for each vaccine type, while our type-specific predictors capture interactions with all vaccine types in a comprehensive way.

Our simulation study revealed how the performance of the proposed predictors depends on the underlying structure of type interactions. The predictors performed best under the pairwise-symmetric multiplicative structure, in which the hazard-based and odds-based predictors are equivalent. The predictors were mostly able to capture the opposing forces of competition and synergy as well as the interplay between interactions in acquisition and clearance. Under other simulated structures, the hazard-based predictors still performed well, while the odds-based predictors performed fairly up to a reasonable degree of asymmetry. As a rule of thumb, symmetric interactions facilitate the prediction of type replacement, while complex and heterogeneous interactions may necessitate more sophisticated predictors that capture details and directions of interactions. For HPV, little is known about the structure of interactions between genotypes, as the very existence of interaction is already difficult to determine. For instance, any imbalance between type-specific prevalence may mask possible asymmetric interactions, and although pooling of multiple types may increase the power of detecting interaction, it may also obscure type-specific patterns. Nevertheless, our simulation study demonstrated that the proposed predictors are robust against various interaction structures.

We cannot ensure the good performance of the proposed predictors under mechanisms of interactions other than the ones we considered. For instance, if the pathogen types interact through natural cross-immunity that is long-lasting, 
infections with different types may be positively associated (i.e. as given by odds ratios greater than one) while there is a risk for type replacement [30, 31]. This shortcoming is inherent to the difficulty to distinguish between susceptible individuals and those who have acquired natural immunity as is the case for HPV infection which induces only a weak antibody response [32]. Nevertheless, if immunity is confined to be type-specific, the predictors work equally well in a two-type model [21], and we envision the proposed predictors to remain applicable also in a multi-type setting. Another mechanism that we did not consider is competition for transmissibility, e.g. through reduction of viral load during coinfection [13-15]. Although competition for transmissibility is not captured by the proposed predictors, it is likely correlated with competition in clearance, as HPV persistence is also determined by viral load [16]. Hence, the predictors may retain good predictive ability even when not all mechanisms of interactions are captured.

Despite the theoretical appeal of the proposed predictors and their promising performance in simulations, some challenges still need to be addressed. First, more extensive simulations are needed to investigate how well the predictors perform with high numbers of pathogen types. Here, simulations were performed with at most seven types, while HPV consists of up to fifteen high-risk types. Second, our method assumes the elimination of all vaccine-targeted types, although some vaccine types or cross-protective types may persist, especially when vaccination coverage is low [4, 5]. If persistence of targeted types would mostly prevent or limit the extent of type replacement, our method may still provide good predictions as a worst-case scenario. Formal analyses are required to sharpen this intuition. Third, it remains to be investigated whether the hazard-based and odds-based predictors can be accurately estimated from limited data. In particular, although the hazard-based predictors performed better in simulations, it is not straightforward which ones are more suitable for the empirical setting. In general, hazardbased estimates are more robust against different sources of bias (e.g. due to population entry and unobserved heterogeneity) [31] but require a larger sample size than the estimation of type-specific point prevalence. In addition, statistical methods need to be developed to deal with possible confounding due to common risk factors, which were neglected in our analysis. In particular, multivariate statistical methods (e.g. GEE and random effect models) may be adapted to adjust for observed heterogeneity or control for unobserved heterogeneity when estimating the new predictors, which are essentially odds and hazard ratios.

For HPV, most epidemiological studies have concluded a low risk of type replacement based on a lack of systematic patterns of negative associations in the co-occurrence of vaccine and non-vaccine types [12, 25]. The methodology presented in this paper may help to translate the observed patterns into explicit predictions of the overall risk of replacement. Additionally, application of our type-specific predictors could discover non-vaccine types with hitherto hidden potentials for type replacement, which are not only determined by their direct interactions with the 
vaccine types but also by the indirect interactions through other non-vaccine types. As the circulation of vaccine types will be further reduced in the near future, more data will become available to validate predictions about the long-term impact of HPV vaccination. The proposed methodology could also be used to give better insights into the underpinnings of type competition in Streptococcus pneumoniae, a pathogen for which replacement has been widely observed after the introduction of the pneumococcal conjugate vaccination [33, 34].

To conclude, we developed a novel methodology for predicting type replacement in a setting of many interacting types. We did so by relating available epidemiological data to the underlying mechanisms of how pathogen types may interact. The proposed predictors may help to better anticipate and understand the impact of vaccination against pathogens with many coexisting (sub)types. 


\section{References}

[1] C. de Martel, M. Plummer, J. Vignat, et al. "Worldwide burden of cancer attributable to HPV by site, country and HPV type". Int J Cancer 141.4 (2017), 664-670.

[2] N. Muñoz, F. X. Bosch, S. de Sanjosé, et al. "Epidemiologic classification of human papillomavirus types associated with cervical cancer". N Engl J Med 348.6 (2003), 518-527.

[3] S. de Sanjose, W. G. V. Quint, L. Alemany, et al. "Human papillomavirus genotype attribution in invasive cervical cancer: a retrospective cross-sectional worldwide study". Lancet Oncol 11.11 (2010), 1048-1056.

[4] D. Mesher, K. Soldan, M. Lehtinen, et al. "Population-level effects of human papillomavirus vaccination programs on infections with nonvaccine genotypes". Emerg Infect Dis 22.10 (2016), 1732-1740.

[5] K. Kavanagh, K. Pollock, A. Potts, et al. "Introduction and sustained high coverage of the HPV bivalent vaccine leads to a reduction in prevalence of HPV 16/18 and closely related HPV types". Br J Cancer 110.11 (2014), 2804-2811.

[6] M. Drolet, É. Bénard, M. C. Boily, et al. "Population-level impact and herd effects following human papillomavirus vaccination programmes: a systematic review and meta-analysis". Lancet Infect Dis 15.5 (2015), 565-580.

[7] J. E. Tota, A. V. Ramanakumar, M. Jiang, et al. "Epidemiologic approaches to evaluating the potential for human papillomavirus type replacement postvaccination". Am J Epidemiol 178.4 (2013), 625-634.

[8] J. O. Lloyd-Smith. "Vacated niches, competitive release and the community ecology of pathogen eradication". Philos Trans R Soc B Biol Sci 368.1623 (2013), 20120150.

[9] M. Safaeian and A. C. Rodriguez. "Invited commentary: multiple human papillomavirus infections and type replacement-anticipating the future after human papillomavirus vaccination". Am J Epidemiol 180.11 (2014), 1076-1081.

[10] M. Saccucci, E. L. Franco, L. Ding, et al. "Non-vaccine-type human papillomavirus prevalence after vaccine introduction: no evidence for type replacement but evidence for cross-protection". Sex Transm Dis 45.4 (2018), 260-265.

[11] J. A. Kahn, D. R. Brown, L. Ding, et al. "Vaccine-type human papillomavirus and evidence of herd protection after vaccine introduction". Pediatrics 130.2 (2012), e249-e256.

[12] J. E. Tota, A. V. Ramanakumar, L. L. Villa, et al. "Evaluation of human papillomavirus type replacement postvaccination must account for diagnostic artifacts: masking of HPV52 by HPV16 in anogenital specimens". Cancer Epidemiol Biomarkers Prev 24.1 (2015), 286-290.

[13] L. F. Xi, Z. R. Edelstein, C. Meyers, et al. "Human papillomavirus types 16 and 18 DNA load in relation to coexistence of other types, particularly those in the same species". Cancer Epidemiol Biomarkers Prev 18.9 (2009), 2507-2512.

[14] R. B. Roden and P. L. Stern. "Opportunities and challenges for human papillomavirus vaccination in cancer". Nat Rev Cancer 18.4 (2018), 240-254.

[15] J. Biryukov and C. Meyers. "Superinfection exclusion between two high-risk human papillomavirus types during a coinfection". J Virol 92.8 (2018), e01993-17.

[16] E. Marra, A. King, E. van Logchem, et al. "Anal HPV 16 and 18 viral load: A comparison between HIV-negative and -positive MSM and association with persistence". J Med Virol 90.1 (2018), 76-83.

[17] M. T. Sofonea, S. Alizon, and Y. Michalakis. "Exposing the diversity of multiple infection patterns". J Theor Biol 419 (2017), 278-289. 
[18] K. K. Thomas, J. P. Hughes, J. M. Kuypers, et al. "Concurrent and sequential acquisition of different genital human papillomavirus types". J Infect Dis 182.4 (2000), 1097-1102.

[19] M. C. Rousseau, J. S. Pereira, J. C. Prado, et al. "Cervical coinfection with human papillomavirus (HPV) types as a predictor of acquisition and persistence of HPV infection". $J$ Infect Dis 184.12 (2001), 1508-1517.

[20] M. Merikukka, M. Kaasila, P. B. Namujju, et al. "Differences in incidence and co-occurrence of vaccine and nonvaccine human papillomavirus types in Finnish population before human papillomavirus mass vaccination suggest competitive advantage for HPV33". Int J Cancer 128.5 (2011), 1114-1119.

[21] I. Man, J. Wallinga, and J. A. Bogaards. "Inferring pathogen type interactions using crosssectional prevalence data: opportunities and pitfalls for predicting type replacement". Epidemiology 29.5 (2018), 666-674.

[22] P. E. Gravitt. "The known unknowns of HPV natural history". J Clin Invest 121.12 (2011), 45934599.

[23] S. L. Ranjeva, E. B. Baskerville, V. Dukic, et al. "Recurring infection with ecologically distinct HPV types can explain high prevalence and diversity". Proc Natl Acad Sci U S A 114.51 (2017), 13573-13578.

[24] J. E. Tota, M. Jiang, A. V. Ramanakumar, et al. "Epidemiologic evaluation of human papillomavirus type competition and the potential for type replacement post-vaccination". PLoS One 11.12 (2016), e0166329.

[25] A. K. Chaturvedi, H. A. Katki, A. Hildesheim, et al. "Human papillomavirus infection with multiple types: pattern of coinfection and risk of cervical disease". J Infect Dis 203.7 (2011), 910-920.

[26] M. Mollers, H. J. Vriend, M. A. van der Sande, et al. "Population- and type-specific clustering of multiple HPV types across diverse risk populations in the Netherlands". Am J Epidemiol 179.10 (2014), 1236-1246.

[27] D. C. Beachler, G. Jenkins, M. Safaeian, et al. "Natural acquired immunity against subsequent genital human papillomavirus infection: a systematic review and meta-analysis". J Infect Dis 213.9 (2015), 1444-1454.

[28] J. Mehtälä, M. Antonio, M. S. Kaltoft, et al. "Competition between Streptococcus pneumoniae strains: implications for vaccine-induced replacement in colonization and disease". Epidemiology (2013), 522-529.

[29] K. Auranen, H. Rinta-Kokko, and M. E. Halloran. "Estimating strain-specific and overall efficacy of polyvalent vaccines against recurrent pathogens from a cross-sectional study". Biometrics 69.1 (2013), 235-244.

[30] D. P. Durham, E. M. Poolman, Y. Ibuka, et al. "Reevaluation of epidemiological data demonstrates that it is consistent with cross-immunity among human papillomavirus types". J Infect Dis 206.8 (2012), 1291-1298.

[31] T. Malagón, P. Lemieux-Mellouki, J. F. Laprise, et al. "Bias due to correlation between times-atrisk for infection in epidemiologic studies measuring biological interactions between sexually transmitted infections: a case study using human papillomavirus type interactions". Am J Epidemiol 184.12 (2016), 873-883.

[32] M. A. Vink, J. Berkhof, J. van de Kassteele, et al. "A bivariate mixture model for natural antibody levels to human papillomavirus types 16 and 18: baseline estimates for monitoring the herd effects of immunization". PLoS One 11.8 (2016), e0161109.

[33] D. R. Feikin, E. W. Kagucia, J. D. Loo, et al. "Serotype-specific changes in invasive pneumococcal disease after pneumococcal conjugate vaccine introduction: a pooled analysis of multiple surveillance sites". PLoS Med 10.9 (2013), e1001517. 
[34] S. Flasche, O. L. P. de Waroux, K. L. O'Brien, et al. "The serotype distribution among healthy carriers before vaccination is essential for predicting the impact of pneumococcal conjugate vaccine on invasive disease". PLoS Comput Biol 11.4 (2015), e1004173.

\section{Appendices of Chapter 5}

\section{A. Equivalence of hazard-based and odds-based predictors under de- tailed balance}

We prove the equivalence of the type-specific hazard-based and odds-based predictors (defined in Equation (5.3) and Equation (5.5) in the main text, respectively) under detailed balance, i.e. assuming that $I_{X} q_{X \rightarrow Y}=I_{Y} q_{Y \rightarrow X}$ for any pair of states $X, Y$. According to definition Equation (5.3),

$$
\begin{aligned}
& H R_{V T, i}=\left(\frac{\sum_{X \in \mathcal{A}_{V T}} I_{X \mid \mathcal{A}_{V T}} q_{X \rightarrow X \cup\{i\}}}{\sum_{X \in \mathcal{A}_{0}} I_{X \mid \mathcal{A}_{0}} q_{X \rightarrow X \cup\{i\}}}\right) /\left(\frac{\sum_{X \in \mathcal{A}_{V T, i}} I_{X \mid \mathcal{A}_{V T, i}} q_{X \rightarrow X \backslash\{i\}}}{\sum_{X \in \mathcal{A}_{i}} I_{X \mid \mathcal{A}_{i}} q_{X \rightarrow X \backslash\{i\}}}\right) \\
& =\left(\frac{\frac{\sum_{X \in \mathcal{A}_{V T}} I_{X} q_{X \rightarrow X \cup\{i\}}}{\sum_{X \in \mathcal{A}_{V T}} I_{X}}}{\frac{\sum_{X \in \mathcal{A}_{0}} I_{X} q_{X \rightarrow X \cup\{i\}}}{\sum_{X \in \mathcal{A}_{0}} I_{X}}}\right) /\left(\frac{\frac{\sum_{X \in \mathcal{A}_{V T, i}} I_{X} q_{X \rightarrow X \backslash\{i\}}}{\sum_{X \in \mathcal{A}_{V T, i}} I_{X}}}{\frac{\sum_{X \in \mathcal{A}_{i}} I_{X} q_{X \rightarrow X \backslash\{i\}}}{\sum_{X \in \mathcal{A}_{i}} I_{X}}}\right),
\end{aligned}
$$

where each of the four aggregate-level hazards are specified as weighted averages of the respective incoming type-specific hazards (see text). Reordering the terms as $\left(\frac{\frac{a_{1}}{a_{2}}}{\frac{b_{1}}{b_{2}}}\right) /\left(\frac{\frac{c_{1}}{c_{2}}}{\frac{d_{1}}{d_{2}}}\right)=\left(\frac{a_{1} \cdot b_{2}}{a_{2} \cdot b_{1}}\right)$. $\left(\frac{c_{2} \cdot d_{1}}{c_{1} \cdot d_{2}}\right)=\left(\frac{a_{1} \cdot d_{1}}{b_{1} \cdot c_{1}}\right) \cdot\left(\frac{b_{2} \cdot c_{2}}{a_{2} \cdot d_{2}}\right)$ gives

$$
H R_{V T, i}=\left(\frac{\sum_{X \in \mathcal{A}_{V T}} I_{X} q_{X \rightarrow X \cup\{i\}} \cdot \sum_{X \in \mathcal{A}_{i}} I_{X} q_{X \rightarrow X \backslash\{i\}}}{\sum_{X \in \mathcal{A}_{0}} I_{X} q_{X \rightarrow X \cup\{i\}} \cdot \sum_{X \in \mathcal{A}_{V T, i}} I_{X} q_{X \rightarrow X \backslash\{i\}}}\right) \cdot\left(\frac{\sum_{X \in \mathcal{A}_{0}} I_{X} \cdot \sum_{X \in \mathcal{A}_{V T, i}} I_{X}}{\sum_{X \in \mathcal{A}_{V T}} I_{X} \cdot \sum_{X \in \mathcal{A}_{i}} I_{X}}\right) .
$$

As each state in $\mathcal{A}_{i}$ can be reached from $\mathcal{A}_{0}$ by adding (acquiring) type $i$, the sum over $\mathcal{A}_{i}$ can be written as a sum over $\mathcal{A}_{0}$ (and analogously for $\mathcal{A}_{V T, i}$ and $\mathcal{A}_{V T}$ ):

$$
H R_{V T, i}=\left(\frac{\sum_{X \in \mathcal{A}_{V T}} I_{X} q_{X \rightarrow X \cup\{i\}} \cdot \sum_{X \in \mathcal{A}_{0}} I_{X \cup\{i\}} q_{X \cup\{i\} \rightarrow X}}{\sum_{X \in \mathcal{A}_{0}} I_{X} q_{X \rightarrow X \cup\{i\}} \cdot \sum_{X \in \mathcal{A}_{V T}} I_{X \cup\{i\}} q_{X \cup\{i\} \rightarrow X}}\right) \cdot\left(\frac{\sum_{X \in \mathcal{A}_{0}} I_{X} \cdot \sum_{X \in \mathcal{A}_{V T, i}} I_{X}}{\sum_{X \in \mathcal{A}_{V T}} I_{X} \cdot \sum_{X \in \mathcal{A}_{i}} I_{X}}\right) .
$$

Finally, invoking detailed balance shows that $H R_{V T, i}$ equals $O R_{V T, i}$ :

$$
\begin{aligned}
& H R_{V T, i}=\left(\frac{\sum_{X \in \mathcal{A}_{V T}} I_{X} q_{X \rightarrow X \cup\{i\}} \cdot \sum_{X \in \mathcal{A}_{0}} I_{X} q_{X \rightarrow X \cup\{i\}}}{\sum_{X \in \mathcal{A}_{0}} I_{X} q_{X \rightarrow X \cup\{i\}} \cdot \sum_{X \in \mathcal{A}_{V T}} I_{X} q_{X \rightarrow X \cup\{i\}}}\right) \cdot\left(\frac{\sum_{X \in \mathcal{A}_{0}} I_{X} \cdot \sum_{X \in \mathcal{A}_{V T, i}} I_{X}}{\sum_{X \in \mathcal{A}_{V T}} I_{X} \cdot \sum_{X \in \mathcal{A}_{i}} I_{X}}\right) \\
& =\left(\frac{\sum_{X \in \mathcal{A}_{V T, i}} I_{X}}{\sum_{X \in \mathcal{A}_{V T}} I_{X}}\right) /\left(\frac{\sum_{X \in \mathcal{A}_{i}} I_{X}}{\sum_{X \in \mathcal{A}_{0}} I_{X}}\right)=O R_{V T, i} .
\end{aligned}
$$




\section{B. Pooled predictor of overall replacement}

We here prove that the odds ratio of overall non-vaccine-type infection $\left(\overline{O R}_{N V T}\right)$, comparing the preand post-vaccination steady states, can be approximated by the product of the odds ratios of nonvaccine-type-specific infections, $\prod_{i \in N V T} \overline{O R}_{i}$, under mutually independent non-vaccine-type infection dynamics. We start by introducing some notations:

- $I_{X}$ : pre-vaccination steady-state prevalence of infection state $X$;

- $I_{X}^{\prime}$ : post-vaccination steady-state prevalence of infection state $X$;

- $E_{i}=\sum_{i \in X} I_{X}$ : pre-vaccination prevalence of type- $i$ infection;

- $E_{i}^{\prime}=\sum_{i \in X} I_{X}^{\prime}$ : post-vaccination prevalence of type- $i$ infection;

- odds $_{i}=E_{i} /\left(1-E_{i}\right)$ : pre-vaccination odds of type- $i$ infection;

- odds $s_{i}^{\prime}=E_{i}^{\prime} /\left(1-E_{i}^{\prime}\right)$ : post-vaccination odds of type- $i$ infection;

- $\overline{O R}_{i}=o d d s_{i} / o d d s_{i}^{\prime}$ : true odds ratio of type-i infection, comparing the pre- and post-vaccination steady states;

- $E_{N V T}=\sum_{|X \cap N V T|>0} I_{X}$ : pre-vaccination overall non-vaccine-type prevalence;

- $E_{N V T}^{\prime}=\sum_{|X \cap N V T|>0} I_{X}^{\prime}$ : post-vaccination overall non-vaccine-type prevalence;

- odd $s_{N V T}=E_{N V T} /\left(1-E_{N V T}\right)$ : pre-vaccination odds of overall non-vaccine-type infection;

- odd $_{N V T}^{\prime}=E_{N V T}^{\prime} /\left(1-E_{N V T}^{\prime}\right)$ : post-vaccination odds of overall non-vaccine-type infection;

- $\overline{O R}_{N V T}=o d d s_{N V T} / o d d s_{N V T}^{\prime}$ : true odds ratio of overall non-vaccine-type infection, comparing the pre- and post-vaccination steady states.

For notational convenience, we have purposefully defined the above odds ratios with the pre-vaccination odds in the numerator and the post-vaccination odds in the denominator. Accordingly, $\overline{O R}_{i}<1$ and $\overline{O R}_{N V T}<1$ characterise type-specific and overall replacement, respectively, corresponding to the way values less than one of $O R_{i}$ and $O R$ predict replacement.

We now prove that $\overline{O R}_{N V T} \approx \prod_{i \in N V T} \overline{O R}_{i}$ for two non-vaccine types. The proof for more than two non-vaccine types proceeds analogously. We start by rewriting odds ${ }_{N V T}$ :

$$
\text { odd } s_{N V T}=\frac{E_{N V T}}{1-E_{N V T}}=\frac{I_{1}+I_{2}+I_{12}}{I_{\emptyset}} .
$$

By assuming independence between the non-vaccine types, i.e. $I_{12}=E_{1} E_{2}$ :

$$
\begin{aligned}
o d d s_{N V T} & =\frac{E_{1}\left(1-E_{2}\right)+\left(1-E_{1}\right) E_{2}+E_{1} E_{2}}{\left(1-E_{1}\right)\left(1-E_{2}\right)} \\
& =\frac{E_{1}}{1-E_{1}}+\frac{E_{2}}{1-E_{2}}+\frac{E_{1} E_{2}}{\left(1-E_{1}\right)\left(1-E_{2}\right)} \\
& =o d d s_{1}+o_{d d}+\text { odds }_{1} \text { odd } s_{2} \\
& =\overline{O R}_{1} \text { odds } s_{1}^{\prime}+\overline{O R}_{2} \text { odds }{ }_{2}^{\prime}+\overline{O R}_{1} \overline{O R}_{2} \text { odds }{ }_{1}^{\prime} \text { odd }_{2}^{\prime} .
\end{aligned}
$$

Similarly, odds $s_{N V T}^{\prime}=o d d s_{1}^{\prime}+o d d s_{2}^{\prime}+o d d s_{1}^{\prime}$ odd $s_{2}^{\prime}$. The true odds ratio for the overall non-vaccinetype infection then is

$$
\overline{O R}_{N V T}=o d d s_{N V T} / o d d s_{N V T}^{\prime}=\frac{\overline{O R}_{1} \text { odd } s_{1}^{\prime}+\overline{O R}_{2} \text { odd } s_{2}^{\prime}+\overline{O R}_{1} \overline{O R}_{2} \text { odds } s_{1}^{\prime} \text { odd } s_{2}^{\prime}}{o d d s_{1}^{\prime}+o d d s_{2}^{\prime}+o d d s_{1}^{\prime} \text { odds } s_{2}^{\prime}},
$$

which has a value less than one when overall type replacement occurs. As the term $\overline{O R}_{1} \overline{O R}_{2}$ appears in the expression of the true odds ratio, $\overline{O R}_{N V T}$, it can be used as a predictor of overall type replacement. How well $\overline{O R}_{N V T}$ and $\overline{O R}_{1} \overline{O R}_{2}$ correspond to one another can be illustrated in the following example. Figure S5.1 shows for which combination of values of $\overline{O R}_{1}$ and $\overline{O R}_{2}$ the predictor, 
$\overline{O R}_{1} \overline{O R}_{2}$, is less than one (the area below the dashed-dotted curve), predicting replacement. The area in which the true odds ratio of overall non-vaccine-type prevalence is less than one (the area below the dashed curve) is seen to coincide, except for small areas indicated by the arrows in the figure. The figure was generated with odd $s_{1}=0.64$ and $o d d s_{2}=0.37$. Other values give similar results. Due to this correspondence, $\overline{O R}_{N V T}$ can be well approximated by $\overline{O R}_{1} \overline{O R}_{2}$.

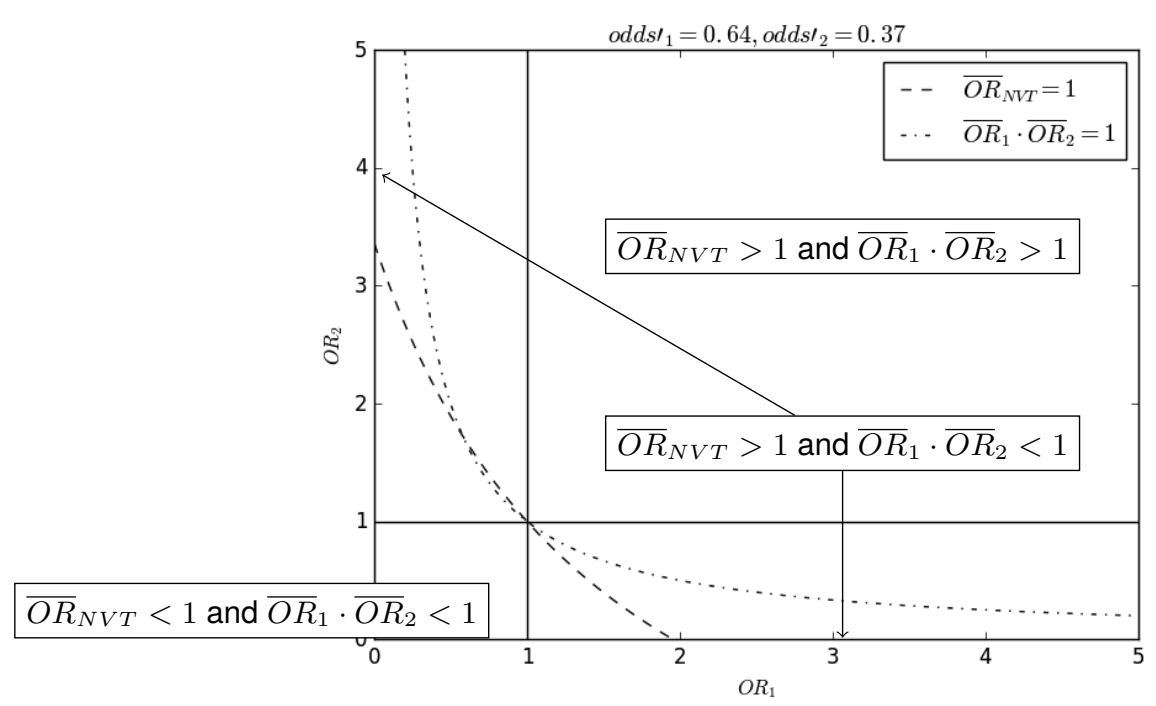

Figure S5.1: Contour plot of $\overline{O R}_{N V T}$ and $\overline{O R}_{1} \overline{O R}_{2}$ with contour lines at $\overline{O R}_{N V T}=1$ and $\overline{O R}_{1} \overline{O R}_{2}=1$, respectively. The values of $o d d_{1}^{\prime}=0.64$ and $o d d_{2}^{\prime}=0.37$ were randomly generated.

As, in addition, each $\overline{O R}_{i}$ can be predicted by $O R_{V T, i}$ (defined in Equation (5.5) in the main text), we envision that $\overline{O R}_{N V T}$ can be well predicted by $\prod_{i \in N V T} O R_{V T, i}$ (defined in Equation (5.7) in the main text). The performance of using $\prod_{i \in N V T} O R_{V T, i}$ to predict overall replacement is evaluated in Section "Simulation results" of the main text.

\section{A multi-type $S$ usceptible-Infected- $S$ usceptible model}

In this section, we describe the transmission model used to simulate the impact of vaccination. The model has $S$ usceptible-Infected-Susceptible dynamics with regard to each of the pathogen types. It allows interactions in both acquisition and clearance. We describe the model's infection states and the respective transition hazards in Section C.1, the pre- and post-vaccination steady states in Section C.2, and three alternative structures of type interactions in Section C.3.

\section{C.1. Infection states and transition hazards}

For a pathogen with $n$ types, the model consists of $2^{n}$ infection states, each defined by the set of types the host is infected with. We denote the set of infection states by $\mathcal{S}$. Acquisitions and clearances of type-specific infection are assumed to be sequential so that only transitions between states that differ 
by infection of one type are possible. Figure 5.1A in the main text shows the eight infection states and the respective transitions for a pathogen with three types.

The baseline hazard for a susceptible individual to acquire type $i$ is $q_{\emptyset \rightarrow i}=c \beta_{i} \sum_{i \in X} I_{X}$, where $c$ is the contact rate, and $\beta_{i}$ is the proportion (probability) of successfully acquiring type $i$ given contact with an individual infected with type $i$. The baseline hazard of clearing type $i$ by a singly infected individual is $q_{i \rightarrow \emptyset}$. The other hazards of acquisition and clearance are given by

$$
\left\{\begin{array}{l}
q_{X \rightarrow X \cup\{i\}}=k_{X i} \cdot q_{\emptyset \rightarrow i}, \\
q_{X \cup\{i\} \rightarrow X}=h_{X i} \cdot q_{i \rightarrow \emptyset},
\end{array}\right.
$$

where $k_{X i}$ and $h_{X i}$ are defined by the underlying structure of type interaction (cf. Section C.3).

\section{C.2. System of ordinary differential equations}

A system of ordinary differential equations describes how the proportions of individuals in each of the model states evolve over time. The equation for state $X$ is

$\frac{d I_{X}}{d t}=\sum_{i \in X} I_{X \backslash\{i\}} q_{X \backslash\{i\} \rightarrow X}-\sum_{i \in X} I_{X} q_{X \rightarrow X \backslash\{i\}}+\sum_{i \notin X} I_{X \cup\{i\}} q_{X \cup\{i\} \rightarrow X}-\sum_{i \notin X} I_{X} q_{X \rightarrow X \cup\{i\}}$,

where the four terms correspond to:

- transition from $I_{X \backslash\{i\}}$ to $I_{X}$ by acquisition of type $i \in X$;

- transition from $I_{X}$ to $I_{X \backslash\{i\}}$ by clearance of type $i \in X$;

- transition from $I_{X \cup\{i\}}$ to $I_{X}$ by clearance of type $i \notin X$;

- transition from $I_{X}$ to $I_{X \cup\{i\}}$ by acquisition of type $i \notin X$.

The pre-vaccination steady state $\left\{I_{X}\right\}_{X \in \mathcal{S}}$ is defined by the condition $\frac{d I_{X}}{d t}=0$. The postvaccination steady state $\left\{I_{X}^{\prime}\right\}_{X \in \mathcal{S}}$ is determined by the condition $\frac{d I_{X}^{\prime}}{d t}=0$ and the condition $I_{X}^{\prime}=0$ for any state containing vaccine types, i.e. $|X \cap V T|>0$. In effect, the post-vaccination steady state is defined as the steady state after the elimination of all vaccine types. Thereby, we implicitly assume a vaccination scheme (i.e. coverage of vaccination and efficacy against the vaccine types) that leads to elimination. Note that, in the model, the non-vaccine-type prevalence in such a post-vaccination steady state is the same regardless of the exact vaccination scheme. As long as the scheme achieves elimination, the system will converge to the unique steady state with only non-vaccine types.

\section{C.3. Multiplicative structures of type interaction}

C.3.1. Pairwise-symmetric and pairwise-asymmetric structures

Under both the pairwise-symmetric and pairwise-asymmetric multiplicative interaction structure Figure S5.2, the hazards of acquisition and clearance in presence of infection with other types are

$$
\left\{\begin{array}{l}
q_{X \rightarrow X \cup\{i\}}=\left(\prod_{j \in X} k_{j i}\right) \cdot q_{\emptyset \rightarrow i}, \\
q_{X \cup\{i\} \rightarrow X}=\left(\prod_{j \in X} h_{j i}\right) \cdot q_{i \rightarrow \emptyset} .
\end{array}\right.
$$

In other words, each (co)infecting type contributes a multiplicative factor to the hazard of acquiring (clearing) the incoming (outgoing) type. Moreover, under the symmetry assumption, $k_{i j}=k_{j i}$ and $h_{i j}=h_{j i}$ for all $i \neq j$. 

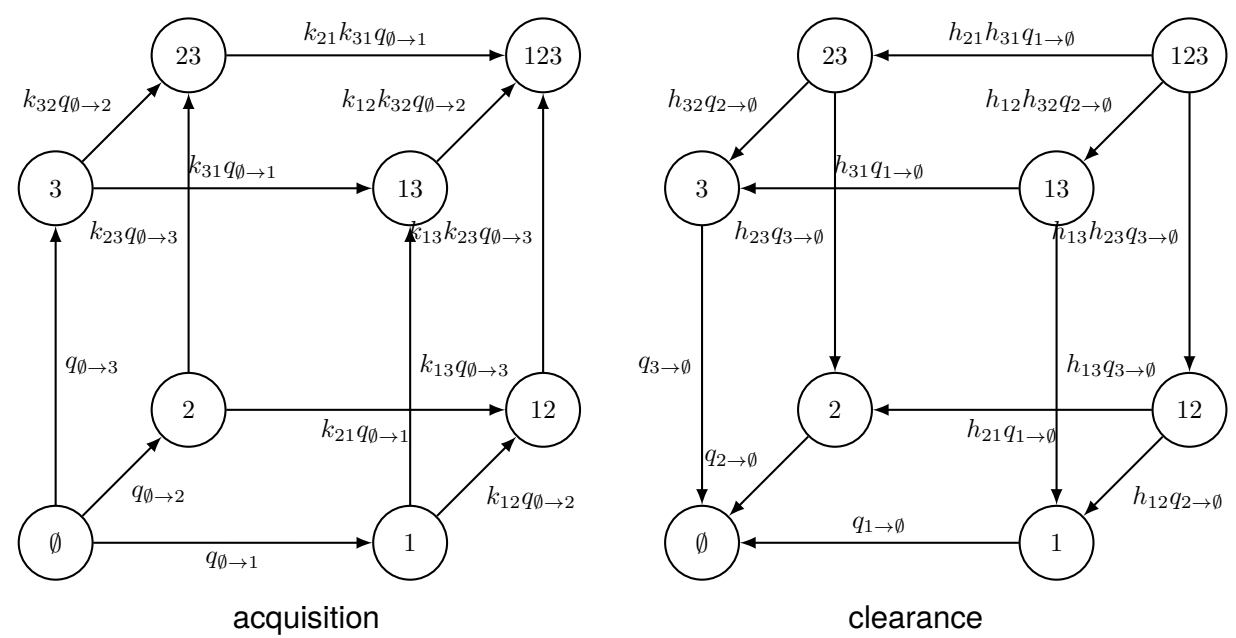

clearance

Figure S5.2: The eight infection states and the respective (left) acquisitions and (right) clearances in a three-type system, under the pairwise-symmetric or pairwise-asymmetric multiplicative structure.

\section{C.3.2. Groupwise-symmetric structure}

The groupwise-symmetric multiplicative structure Figure S5.3 assumes that the multiplicativity acts on groups of types instead of individual types. Given a system with $n$ types and $m \leq n$ groups of types, $A_{1}, A_{2}, \ldots, A_{m}$. Suppose that type $i$ belongs to group $A_{l}$. The hazards of acquiring and clearing type $i$ then are

$$
\left\{\begin{array}{l}
q_{X \rightarrow X \cup\{i\}}=\left(\prod_{j=1}^{m} \mathbb{1}\left\{\left|X \cap A_{j}\right|>0\right\} \cdot k_{j l}\right) \cdot q_{\emptyset \rightarrow i}, \\
q_{X \cup\{i\} \rightarrow X}=\left(\prod_{j=1}^{m} \mathbb{1}\left\{\left|X \cap A_{j}\right|>0\right\} \cdot h_{j l}\right) \cdot q_{i \rightarrow \emptyset} .
\end{array}\right.
$$

The symmetry assumption now reads $k_{j l}=k_{l j}$ and $h_{j l}=h_{l j}$ for all between-group interaction parameters. Furthermore, we abbreviate all within-group interaction parameters $k_{j j}$ and $h_{j j}$ by $k_{j}$ and $h_{j}$, respectively.

Here in the electronic supplementary material, we index groups with numbers, whereas alphabets are used in the main text. The example of the main text reads as follows with the numerical notation: in a four-type system with groups $A_{1}=\{1,2\}, A_{2}=\{3,4\}$, and interaction parameters $k_{1}, k_{2}$ (within groups), $k_{12}=k_{21}$ (between groups), the acquisition hazards of type 1 from state 23 and 234 are both $k_{1} k_{21} q_{\emptyset \rightarrow 1}$.

\section{Detailed balance under the pairwise-symmetric multiplicative struc- ture}

We here prove the pairwise-symmetric multiplicative structure as defined in Section $C$ satisfies the detailed balance property, i.e. $I_{X} q_{X \rightarrow Y}=I_{Y} q_{Y \rightarrow X}$ for any pair of states $X, Y$ in the state space $\mathcal{S}$. The proof is based on first proposing a possible steady-state distribution over $\{X\}_{X \in S}$ and then showing that detailed balance holds for this distribution. As detailed balance implies global balance, the distribution is the unique steady-state distribution [1]. 

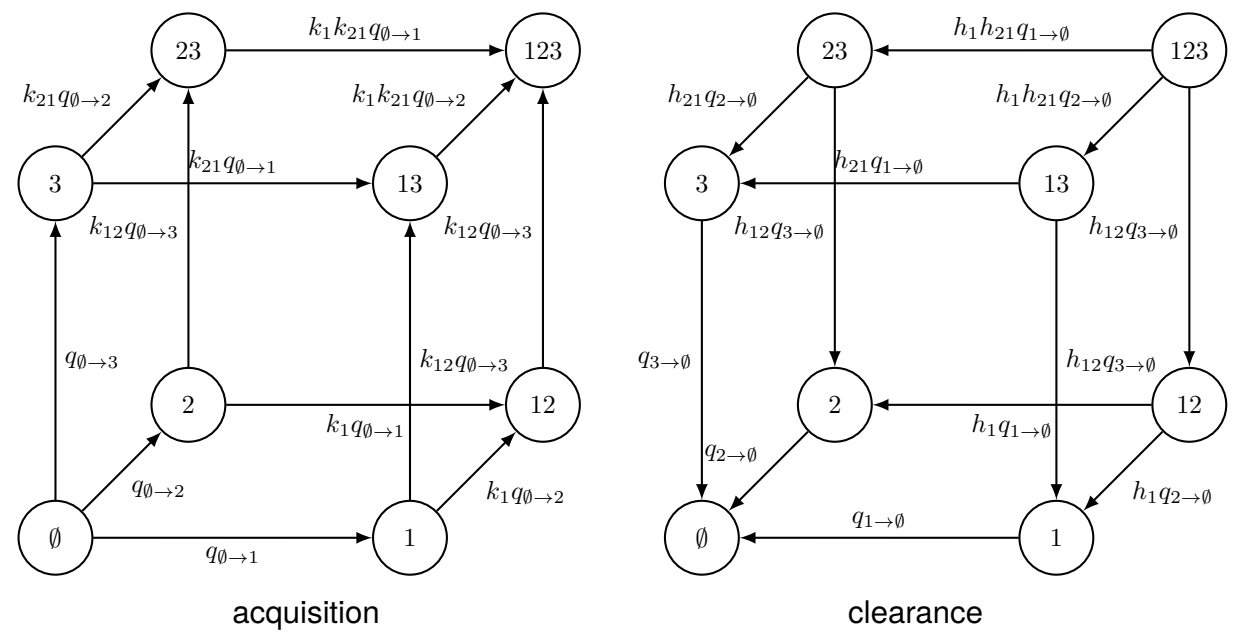

Figure S5.3: The eight infection states and the respective (left) acquisitions and (right) clearances in a three-type system, under the groupwise-symmetric multiplicative structure with groups $A_{1}=\{1,2\}, A_{2}=\{3\}$. Here, we index groups with numbers, whereas alphabets are used in the main text.

We propose the following expression for the steady-state distribution:

$$
I_{X}=\left(\prod_{i \in X} q_{\emptyset \rightarrow i}\right) \cdot\left(\prod_{i \notin X} q_{i \rightarrow \emptyset}\right) \cdot\left(\frac{\prod_{i<j \in X} k_{i j}}{\prod_{i<j \in X} h_{i j}}\right) / C, X \in \mathcal{S} .
$$

Here, $k_{i j}$ and $h_{i j}$ are the interaction parameters, and $C$ is a normalizing constant. Take any pair of 
states that differ with regard to the status of one type exactly, i.e. $X$ and $Y=X \cup\{e\}$ :

$$
\begin{aligned}
\frac{I_{Y}}{I_{X}}= & \frac{\left(\prod_{i \in Y} q_{\emptyset \rightarrow i}\right) \cdot\left(\prod_{i \notin Y} q_{i \rightarrow \emptyset}\right) \cdot\left(\frac{\prod_{i<j \in Y} k_{i j}}{\prod_{i<j \in Y} h_{i j}}\right) / C}{\left(\prod_{i \in X} q_{\emptyset \rightarrow i}\right) \cdot\left(\prod_{i \notin X} q_{i \rightarrow \emptyset}\right) \cdot\left(\frac{\prod_{i<j \in X} k_{i j}}{\prod_{i<j \in X} h_{i j}}\right) / C} \\
= & \frac{q_{\emptyset \rightarrow e} \cdot\left(\prod_{i<j \in Y} k_{i j}\right) \cdot\left(\prod_{i<j \in X} h_{i j}\right)}{q_{e \rightarrow \emptyset} \cdot\left(\prod_{i<j \in X} k_{i j}\right) \cdot\left(\prod_{i<j \in Y} h_{i j}\right)} \\
= & \frac{q_{\emptyset \rightarrow e} \cdot\left(\prod_{i<j \in X} k_{i j}\right) \cdot\left(\prod_{i<j \in X} h_{i j}\right) \cdot\left(\prod_{i \in X} k_{i e}\right)}{q_{e \rightarrow \emptyset} \cdot\left(\prod_{i<j \in X} k_{i j}\right) \cdot\left(\prod_{i<j \in X} h_{i j}\right) \cdot\left(\prod_{i \in X} h_{i e}\right)} \\
= & \frac{q_{\emptyset \rightarrow e} \cdot\left(\prod_{i \in X} k_{i e}\right)}{q_{e \rightarrow \emptyset} \cdot\left(\prod_{i \in X} h_{i e}\right)} \\
= & \frac{q_{X \rightarrow Y}}{q_{Y \rightarrow X}} .
\end{aligned}
$$

For any pair of states that are not connected, $q_{X \rightarrow Y}=q_{Y \rightarrow X}=0$ so that $I_{X} q_{X \rightarrow Y}=I_{Y} q_{Y \rightarrow X}$ holds trivially. Hence, we have proved that the proposed distribution satisfies the detailed balance property.

\section{E. Simulation procedure}

For any given simulation setting (e.g. the pairwise-symmetric multiplicative structure with $|V T|=1$ and $|N V T|=2), N=10000$ different parameter sets were randomly generated. The varying model parameters were the interaction parameters (see below) and the type-specific transmissibilities $\left(\beta_{i}\right.$ as defined in Section C.1), while the baseline hazards of clearance were fixed, i.e. $q_{i \rightarrow \emptyset}=1$ for all type $i$. Of note, the results in the main text were generated with no interaction in clearance, i.e. $h_{i j}=1$. See Section $\mathrm{H}$ for the simulation results obtained in the general case with interaction in both acquisition and clearance. The parameter values and ranges used are given in Table S5.1.

The varied interaction parameters (as defined in Section C.3) under different interaction structures were generated as follows:

- Pairwise-symmetric multiplicative structure: altogether $n(n-1) / 2$ parameters $k_{i j}$ for $i<j$;

- Pairwise-asymmetric multiplicative structure: altogether $n(n-1)$ parameters $k_{i j}^{\prime}$ for $i \neq j$. These asymmetric interaction parameters were obtained by either perturbing the interaction parameters generated under the pairwise-symmetric multiplicative structure, or by generating $k_{i j}^{\prime}, k_{j i}^{\prime}$ independently of each other. Perturbation of the pairwise-symmetric parameters is done by adding or subtracting $\epsilon / 2$ on a log scale. Which one of $k_{i j}^{\prime}$ and $k_{j i}^{\prime}$ was derived from addition (and which one from subtraction) was decided randomly with 50/50 chance. This construction ensures the order of $k_{i j}^{\prime}$ and $k_{j i}^{\prime}$ to be random, while $\left|\log \left(k_{i j}^{\prime}\right)-\log \left(k_{j i}^{\prime}\right)\right|=\epsilon$ fixed. Hence, the ratio between any pair of reverse interaction parameters $k_{i j}^{\prime} / k_{j i}^{\prime}\left(\right.$ or $\left.k_{j i}^{\prime} / k_{i j}^{\prime}\right)$ was $e^{\epsilon}=1.22,1.65$. The applied values of $\epsilon$ were 0.2 and 0.5 . We also considered a maximal amount of asymmetry by generating $k_{i j}^{\prime}, k_{j i}^{\prime}$ independently of each other;

- Groupwise-symmetric multiplicative structure: the number of interaction parameters depended 
on the randomly generated number of groups $m \leq n$. With $m$ groups, the numbers of parameters were $m$ (for $k_{i}$ ) and $m(m-1) / 2$ (for $k_{i j}$ where $i<j$ ).

Interaction parameters under the pairwise-symmetric and the groupwise-symmetric multiplicative structure were generated uniformly on a log scale in the interval $(1 / 3,3)$ by means of Latin hypercube sampling, likewise for the pairwise-asymmetric multiplicative structure when generating $k_{i j}^{\prime}, k_{j i}^{\prime}$ independently. The type-specific transmissibilities were generated uniformly in the interval $(2.5,3.5)$ also by means of Latin hypercube sampling.

Note that some generated parameter sets were excluded to retain meaningful simulations. Given a number of types in a setting, we included only parameter sets that allowed all types in the model to be endemic in the pre-vaccination steady state.

For each included parameter set, the following steps were executed:

- simulate the pre-vaccination steady state (as defined in Section C.2) and compute predictors $H R_{V T, i}, H R, O R_{V T, i}$ and $O R$ to predict type-specific and overall replacement;

- simulate the 'true' outcome of vaccination at the post-vaccination steady state (as defined in Section C.2) and compare it to the pre-vaccination predictions.

After simulating all parameter sets, the proportion of corrected predictions is computed for the typespecific and overall outcomes. When there were multiple non-vaccine types, the type-specific performance was first defined and computed for each non-vaccine type separately. As the interactions between different types were generated randomly, the performance of each non-vaccine type should be identical on average. Hence, we computed the numbers shown in Figure 5.2 of the main text and in Figure S5.5 by averaging over the performance of all non-vaccine types.

Table S5.1: Parameter values or ranges used in simulations. $\dagger$ As the per capita contact rate $c$ and acquisition probabilities $\beta_{i}$ always appear in product. The value of $c$ is absorbed in $\beta_{i}$ for notational convenience, which now may attain values above one.

\begin{tabular}{l||l}
\hline Parameter & Values or range \\
\hline Number of types $(n)$ & $2,3, \cdots, 7$ \\
Per capita contact rate $(c)$ & $\dagger$ \\
Probability of successfully acquiring type $i$, & Uniformly distributed between 2.5 and 3.5 for all type $i \dagger$ \\
$\quad$ given contact with infected $\left(\beta_{i}\right)$ & \\
Per capita clearance rate of type $i\left(\mu_{i}\right)$ & 1 for all type $i$ \\
Interaction parameters in acquisition $\left(k_{i j}\right)$ & Uniformly distributed between $1 / 3$ and 3 on a log scale \\
Interaction parameters in clearance $\left(h_{i j}\right)$ & Uniformly distributed between $1 / 3$ and 3 on a log scale \\
Parameter for asymmetric interactions $(\epsilon)$ & $0.2,0.5$ \\
\hline
\end{tabular}




\section{F. Decomposition into direct and indirect interactions}

We here rewrite predictor $H R_{1,3}$ Equation (5.3) into expression Equation (5.9) under the pairwisesymmetric multiplicative structure with only interaction in clearance by decomposing expression Equation (5.3) into direct and indirect interactions.

According to definition (3),

$$
\begin{aligned}
H R_{1,3} & =\frac{I_{1 \mid\{1,12\}} q_{1 \rightarrow 13}+I_{12 \mid\{1,12\}} q_{12 \rightarrow 123}}{I_{\emptyset \mid\{\emptyset, 2\}} q_{\emptyset \rightarrow 3}+I_{2 \mid\{\emptyset, 2\}} q_{2 \rightarrow 23}} \\
& =\frac{\frac{I_{1}}{I_{1}+I_{12}} q_{1 \rightarrow 13}+\frac{I_{12}}{I_{1}+I_{12}} q_{12 \rightarrow 123}}{\frac{I_{\emptyset}}{I_{\emptyset}+I_{2}} q_{\emptyset \rightarrow 3}+\frac{I_{2}}{I_{\emptyset}+I_{2}} q_{2 \rightarrow 23}} \\
& =\left(\frac{I_{1} q_{1 \rightarrow 13}+I_{12} q_{12 \rightarrow 123}}{I_{1}+I_{12}}\right) /\left(\frac{I_{\emptyset} q_{\emptyset \rightarrow 3}+I_{2} q_{2 \rightarrow 23}}{I_{\emptyset}+I_{2}}\right) \\
& =\left(\frac{I_{1} k_{13} q_{\emptyset \rightarrow 3}+I_{12} k_{13} k_{23} q_{\emptyset \rightarrow 3}}{I_{1}+I_{12}}\right) /\left(\frac{I_{\emptyset} q_{\emptyset \rightarrow 3}+I_{2} k_{23} q_{\emptyset \rightarrow 3}}{I_{\emptyset}+I_{2}}\right) \\
& =k_{13} \cdot\left[\left(\frac{I_{1}+I_{12} k_{23}}{I_{1}+I_{12}}\right) /\left(\frac{I_{\emptyset}+I_{2} k_{23}}{I_{\emptyset}+I_{2}}\right)\right] \\
& =k_{13} \cdot\left[\frac{\left(I_{1}+I_{12} k_{23}\right) \cdot\left(I_{\emptyset}+I_{2}\right)}{\left(I_{1}+I_{12}\right) \cdot\left(I_{\emptyset}+I_{2} k_{23}\right)}\right] .
\end{aligned}
$$

The next step is to rewrite $I_{1}$ and $I_{12}$ using the lack of interaction in clearance and the detailed balance property (i.e. $I_{X} q_{X \rightarrow Y}=I_{Y} q_{Y \rightarrow X}$ as proved in Section D):

$$
\begin{gathered}
I_{1}=I_{\emptyset} \frac{q_{\emptyset \rightarrow 1}}{q_{1 \rightarrow \emptyset}}, \\
I_{12}=I_{2} \frac{q_{2 \rightarrow 12}}{q_{12 \rightarrow 2}}=I_{2} \frac{k_{12} q_{\emptyset \rightarrow 1}}{q_{12 \rightarrow 2}}=I_{2} k_{12} \frac{q_{\emptyset \rightarrow 1}}{q_{1 \rightarrow \emptyset}} .
\end{gathered}
$$

Using the above equalities, the equivalence of expressions (3) and (9) can be shown:

$$
\begin{aligned}
H R_{1,3} & =k_{13} \cdot\left[\frac{\left(I_{\emptyset} \frac{q_{\emptyset \rightarrow 1}}{q_{1 \rightarrow \emptyset}}+I_{2} k_{12} \frac{q_{\emptyset \rightarrow 1}}{q_{1 \rightarrow \emptyset}} k_{23}\right) \cdot\left(I_{\emptyset}+I_{2}\right)}{\left(I_{\emptyset} \frac{q_{\emptyset \rightarrow 1}}{q_{1 \rightarrow \emptyset}}+I_{2} k_{12} \frac{q_{\emptyset \rightarrow 1}}{q_{1 \rightarrow \emptyset}}\right) \cdot\left(I_{\emptyset}+I_{2} k_{23}\right)}\right] \\
& =k_{13} \cdot\left[\frac{\left(I_{\emptyset}+I_{2} k_{12} k_{23}\right) \cdot\left(I_{\emptyset}+I_{2}\right)}{\left(I_{\emptyset}+I_{2} k_{12}\right) \cdot\left(I_{\emptyset}+I_{2} k_{23}\right)}\right] .
\end{aligned}
$$




\section{G. Two scenarios of incorrect prediction}

Here, we show two simulated scenarios of incorrect prediction in a three-type system with $V T=$ $\{1\}$ and $N V T=\{2,3\}$, under the pairwise-symmetric multiplicative structure and only interaction in acquisition. In Figure S5.4 the steady-state prevalence is shown for increasing effective vaccine coverage. In the simulation, the effective vaccine coverage was implemented as a reduction factor that acts on the acquisition hazard of the vaccine types. In both scenarios, elimination of the vaccine type was reached at an effective vaccine coverage of around $60 \%$.

Figure S5.4A shows a scenario with $k_{12}=0.5, k_{13}=0.9$ and $k_{23}=0.4$. The predictor for type 3 indicated replacement $\left(H R_{1,3}=0.92<1\right)$, while replacement did not occur. Studying the decomposition of $H R_{1,3}$ into direct and indirect interactions (see expression (9) in the main text) shows that the component for direct (indirect) interaction indicated the tendency of promoting (preventing) replacement. However, the resulting $H R_{1,3}$ predicted replacement, while the indirect interaction was actually stronger, preventing replacement.

Figure S5.4B shows a scenario with $k_{12}=1.4, k_{13}=0.8$ and $k_{23}=0.2$. The overall predictor indicated replacement $H R=H R_{1,2} \cdot H R_{1,3}=1.46 \cdot 0.66=0.96<1$, while replacement did not occur. However, the overall non-vaccine-type prevalence decreased only slightly.
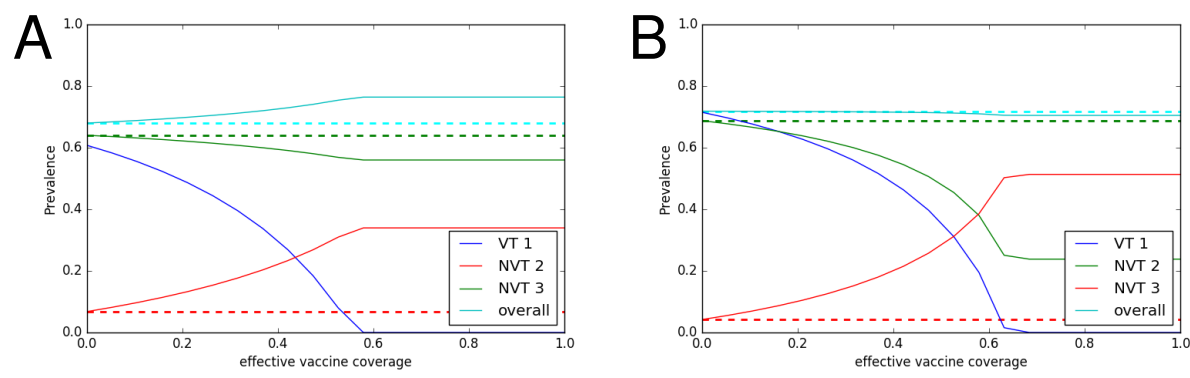

Figure S5.4: Two scenarios of incorrect prediction in a three-type system with $V T=\{1\}$ and $N V T=\{2,3\}$ under the pairwise-symmetric multiplicative structure and with only interaction in acquisition. The steady-state prevalence is shown for increasing effective vaccine coverage. The prevaccination prevalence is shown by dashed lines, whereas the post-vaccination prevalence is shown by solid lines at values of the effective vaccine coverage above $60 \%$. A) A scenario with opposing direct and indirect effects. The predictor for type 3 indicated replacement incorrectly. B) A scenario with modest decrease in the overall non-vaccine-type prevalence, while the overall predictor indicated replacement incorrectly. 


\section{H. Simulation results with interactions in acquisition and clearance}

In this section, we present the simulation results obtained from the model including interaction in clearance, in addition to that in acquisition. It was simulated following the same procedure as outlined in Section E. Each parameter set was expanded by interaction parameters for clearance, which were generated in the same manner as the corresponding interaction parameters for acquisition. For instance, the parameter sets under the pairwise-symmetric multiplicative structure each included $n(n-1) / 2$ parameters $h_{i j}$ that were uniformly generated on a log scale in the interval $(1 / 3,3)$ by means of Latin hypercube sampling. Note that for the groupwise-symmetric structure, the same groups were used to define interactions in acquisition and clearance.

The performance of predictors $H R_{V T, i}, H R, O R_{V T, i}$, and $O R$ under the alternative multiplicative structures for interactions are shown in Figure S5.5 
Pairwise-symmetric with interaction in clearance:

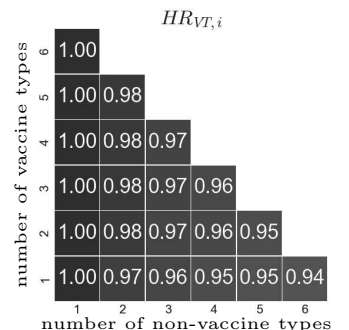

Groupwise-symmetric with interaction in clearance:

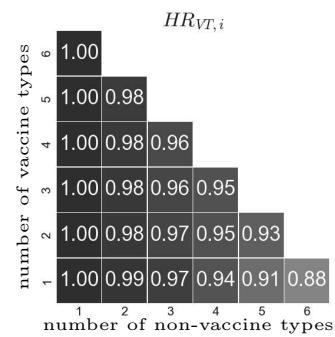

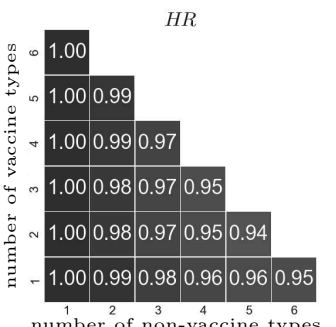

$H R$

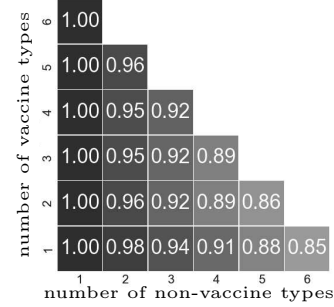

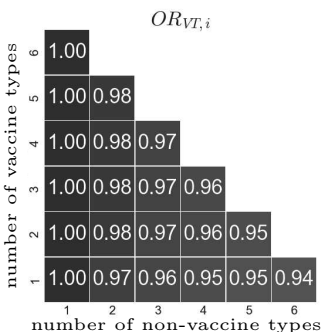

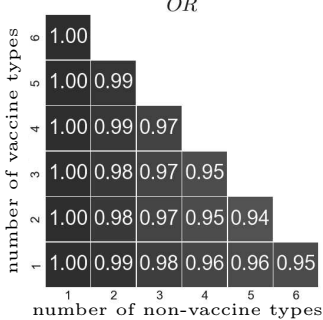

Mildly pairwise-asymmetric $(\epsilon=0.2)$ with interaction in clearance :
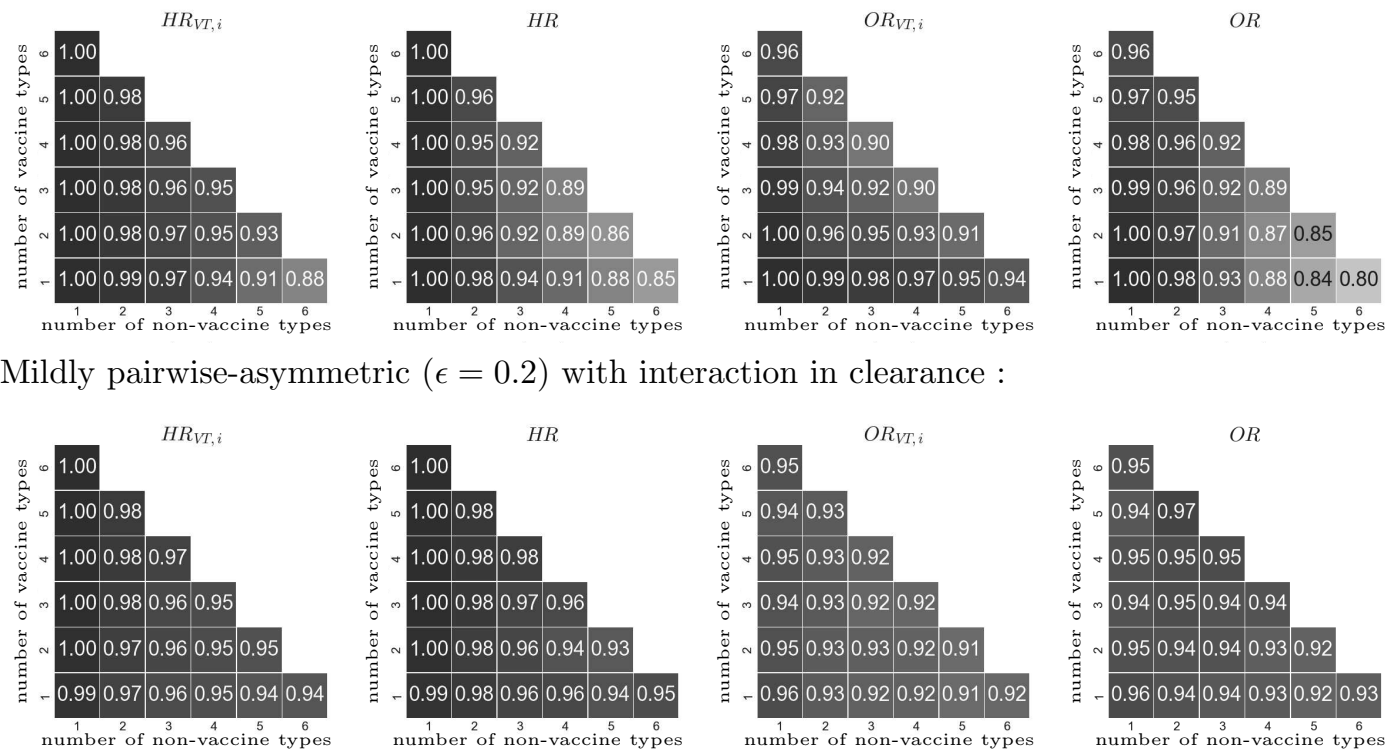

Moderately pairwise-asymmetric $(\epsilon=0.5)$ with interaction in clearance:
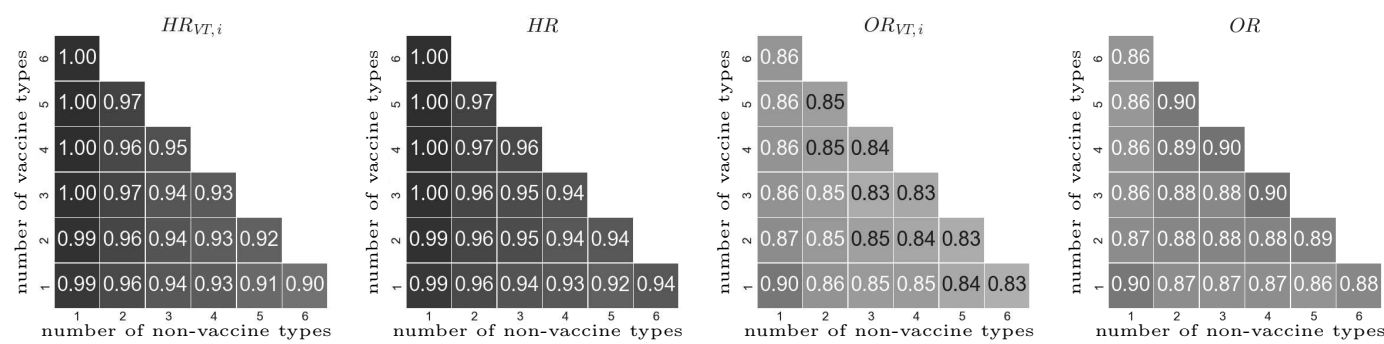

Strongly pairwise-asymmetric with interaction in clearance:
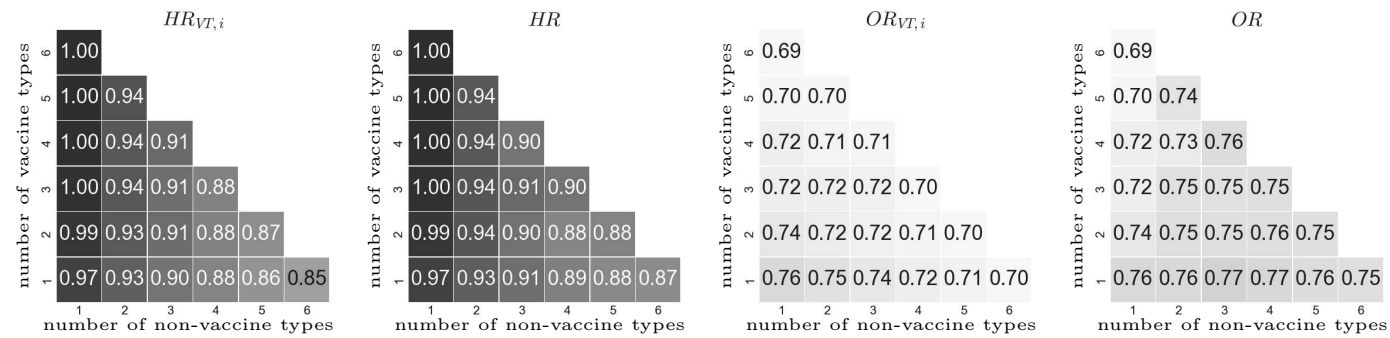

Figure S5.5: Performance (proportion of correct predictions among all generated parameter sets) of predictors $H R_{V T, i}, H R, O R_{V T, i}$, and $O R$ under alternative multiplicative structures for interactions in acquisition and clearance. Row 1: pairwise-symmetric. Row 2: groupwise-symmetric. Rows 3-5: increasing pairwise-asymmetric with increasing values of $\epsilon$ for more asymmetry between pairs of reciprocal interaction parameters. Performance of $H R_{V T, i}$ and $O R_{V T, i}$ were obtained by averaging over the performance of each non-vaccine type $i$. 


\section{Chapter 6}

\section{Human papillomavirus genotype replacement: still too early to tell?}

This chapter has been published as: I. Man, S. Vänskä, M. Lehtinen, and J. A. Bogaards. "Human papillomavirus genotype replacement: still too early to tell?". J Infect Dis, jiaa032 (2019), doi: 10.1093/infdis/jiaa032. 


\section{Abstract}

\section{Background}

Although human papillomavirus (HPV) vaccines are highly efficacious in protecting against HPV infections and related diseases, vaccination may trigger replacement by non-targeted genotypes if these compete with the vaccine-targeted types. HPV genotype replacement has been deemed unlikely based on the lack of systematic increases in non-vaccine-type prevalence in the first decade post-vaccination and on the presence of cross-protection for some non-vaccine types.

\section{Methods}

To investigate whether type replacement can be inferred from early post-vaccination surveillance, we constructed a transmission model in which a vaccine and a nonvaccine type compete through infection-induced cross-immunity. We simulated scenarios of different levels of cross-immunity and vaccine-induced cross-protection to the non-vaccine type. We then examined whether commonly used measures correctly indicate type replacement in the long run.

\section{Results}

Type replacement is a trade-off between cross-immunity and cross-protection; cross-immunity leads to type replacement unless cross-protection were strong enough. With weak cross-protection, the non-vaccine-type prevalence may initially decrease before rebounding into type replacement, exhibiting a honeymoon period. Importantly, vaccine effectiveness for non-vaccine types is inadequate for indicating type replacement.

\section{Conclusions}

Although post-vaccination surveillance thus far is reassuring, it is still too early to preclude type replacement. Monitoring of non-vaccine-types remains pivotal in gauging population-level impacts of HPV vaccination. 


\section{Introduction}

Papillomaviruses are ancient DNA viruses with high diversity in mammalian hosts. Human papillomaviruses (HPV) are classified into genotypes (generally termed "types"), defined by $>10 \%$ DNA sequence divergence from one another in the L1 capsid gene [1]. Sexually transmitted HPV types are causally linked to anogenital and oropharyngeal cancers in men and women, which attribute to almost $5 \%$ of the 14 million new cancer cases occurring annually worldwide [2, 3]. HPV vaccination thus provides a great opportunity to largely reduce the global cancer burden and may even eliminate cervical cancer as a public health problem [4].

Currently, three licensed HPV vaccines are available, constructed using L1 capsid antigens of two, four, and nine HPV genotypes, respectively [2]. The three vaccines are highly efficacious in preventing HPV infections with the corresponding vaccine types, all including high-risk types HPV 16 and 18, which cause the majority of HPV-related cancers [5, 6]. The quadrivalent and nonavalent vaccines also target low-risk types HPV 6 and 11, which are primarily associated with anogenital warts. In addition, the nonavalent vaccine includes five other high-risk types, HPV 31, 33, 45, 52 and 58, while the quadrivalent and bivalent vaccines reportedly provide partial cross-protection, predominantly against HPV 31 , and HPV 31, 33 and 45, respectively [5-10].

As strain-specific vaccination against other multi-strain pathogens, such as adenovirus and Streptococcus pneumoniae, has caused an increased circulation of some non-vaccine-targeted strains, concerns about HPV type replacement have also been raised [11,-15]. The prevailing assumption behind strain replacement is the competition between vaccine-targeted and non-vaccine strains so that vaccination releases non-vaccine strains from competition as a side-effect of eliminating the vaccine-targeted ones [12]. There are empirical findings supporting mutual suppression of viral load between HPV types during coinfection within hosts and competitive exclusion within cells [16, 17]. An evoked immune response that clears infection with a given HPV type might also yield enhanced clearance of infection with other types by activating antigen-presenting cells or cross-immunity. In contrast, multiple epidemiological studies on pre-vaccination HPV (co)infection data reported a lack of systematic negative associations between vaccine-targeted and non-vaccine types, presumed to be indicative of a lack of competitive interactions [18]. However, subsequent modelling studies demonstrated that positive associations might instead reflect the existence of natural cross-immunity, entailing a risk for type replacement [19, 20].

In the first decade following the introduction of HPV vaccination, surveillance data worldwide have shown a slight increase in the pooled prevalence of high-risk non-vaccine types not including HPV 31, 33 and 45 [8]. This increase might reflect an increasing trend in sexual activities or an apparently increased detection of 
non-vaccine types, which were previously masked by infections of HPV 16 and 18 . Alternatively, non-vaccine HPV types could be occupying the ecological niche created by reducing infections of HPV 16 and 18. Type-specific trends of non-vaccine HPV types, however, are more heterogeneous across settings, without consistent signals of type replacement. In addition, negative vaccine effectiveness has been reported for various non-vaccine types (e.g. HPV 40, 42, 43, 54, 56 and 59), but mostly without statistical significance, possibly due to limited power of monitoring studies[10, 21]. In a large-scale community-randomised study with 50\% vaccination coverage, although no consistent patterns of HPV type replacement were observed, the prevalence of HPV 39 and 51 did increase in HPV-vaccinated communities [22]. Taken together, approximately 10 years have elapsed since the wide-scale implementation of HPV vaccines, but the likelihood of type replacement remains ambiguous.

In this paper, we investigate to what extent type replacement can be inferred from early post-vaccination surveillance data. We construct a transmission model in which two HPV types, one vaccine type and one non-vaccine type, compete through naturally acquired, i.e. infection-induced, cross-immunity. Short- and long-term trends of non-vaccine-type prevalence are derived. From the shortterm trends, we compute measures commonly used to evaluate type replacement in real-life surveillance, such as prevalence ratios relative to pre-vaccination periods and vaccine effectiveness for non-vaccine types. These are then compared to the long-term trends. We investigate how the occurrence and timing of type replacement depend on the model parameters and show that, in some cases, observations of initial decreases in non-vaccine-type prevalence and positive vaccine effectiveness may not preclude type replacement in the long run.

\section{Methods}

\section{Model structure}

We constructed a heterosexual compartmental transmission model with two competing HPV types, one vaccine type (VT) and one non-vaccine type (NVT). In the model, individuals of both sexes are divided into two risk groups, distinguished by the rates at which new sexual partners of the opposite sex are contacted. Within each risk group, individuals have the same age-dependent contact rates and prefer partners from the same risk group and of a similar age. We assumed a constant influx of individuals entering the model population at age 10 years and leaving at age 70 years.

We assumed susceptible-infected-recovered-susceptible (SIRS) dynamics for both types (Figure S6.2), in accordance with most heterosexual transmission models for studying population impact of HPV vaccination [23]. Individuals enter the 
population being susceptible to both types and may subsequently undergo events of acquisition, clearance, and waning of natural immunity.

Unvaccinated individuals acquire infection with type $i(=v t, n v t)$ at a rate that is proportional to the rate of new contacts with individuals infected with type $i$. Upon established contact, the transmission probability is $\beta_{i}$. If vaccinated, the infectionacquisition rate is reduced by the type-specific vaccine efficacy $\theta_{i}$. The vaccine is assumed to be fully protective against the VT $\left(\theta_{v t}=100 \%\right)$ and may induce partial protection against the NVT $\left(\theta_{n v t}<100 \%\right)$. Acquired infections of type $i$ are cleared at rate $\mu_{i}$. Clearance induces natural type-specific immunity, inhibiting reinfection with the same type. Immunity wanes at rates $\gamma_{f}$ and $\gamma_{m}$ in females and males, respectively, after which the individual again becomes susceptible to that type.

Between-type competition is modelled by natural cross-immunity. During the period of type-specific immunity, cross-immunity is assumed to accelerate the clearance of the other type by a factor $h \geq 1$, without blocking infection. Crossimmunity is lost simultaneously with the waning type-specific immunity. For simplicity, we only consider the symmetric case in which does not depend on the type. Modelled as such, the two types disadvantage one another through crossimmunity, whereby parameter $h$ regulates the strength of between-type competition, with higher values corresponding to stronger competition. See Appendix A for a detailed description of the model structure.

\section{Parametrisation and scenarios}

The sexual contact rates and mixing distribution were based on the Dutch contact patterns (Figures S6.3-4) [24]. The waning rates of natural immunity were $\gamma_{f}=0.1$ and $\gamma_{m}=1$ per year, corresponding to mean durations of 10 years in women and 1 year in men, respectively. These differences were chosen to reflect varying natural histories of HPV infection by sex and anatomic site; notably, cervico-vaginal HPV infection likely induces some level of systemic immunity, whereas such protection is less obvious in men [25, 26]. To explore scenarios of different levels of competition, we used various pre-fixed values of $h(=1,1.33, \ldots, 3)$. For each value of $h$, other parameters were obtained by calibrating to data of age-specific pre-vaccination HPV prevalence of females in the Netherlands [27]. Data for the VT were the prevalence of HPV-16, which attains its maximal value of approximately $8 \%$ around age 23 years. For the NVT, we assumed a prevalence that is four times lower.

We implemented a girls-only vaccination programme targeting 12-year-olds with $95 \%$ uptake, complemented by a catch-up programme up to age 18 years at the introduction of vaccination (Figure S6.10A). This setting roughly mimics HPV vaccination programmes in most Western countries, albeit with an overoptimistic vaccine uptake to study the appearance of type replacement. For sim- 
plicity, we assumed no difference in uptake due to risk-group stratification. To explore scenarios of different levels of cross-protection for the NVT, we considered $\theta_{n v t}=0,10, \cdots, 50 \%$.

We performed sensitivity analyses regarding shorter natural immunity, lower vaccination coverage in girls, and higher coverage by adding boys' vaccination (Figure S6.10B). See Appendix B for the calibration process, parameter values used, and description of the sensitivity analyses.

\section{Measures for type replacement}

We defined type replacement as an increase in the prevalence of NVT infection in the post-vaccination period as compared to the pre-vaccination equilibrium. Type replacement evaluated in the long run and short-term were defined as follows.

Final type replacement - To evaluate the population impact of vaccination on NVT infection in the long run, we utilised $P R^{\text {final }}$, which compares the NVT prevalence among all women in the post-vaccination equilibrium to the pre-vaccination equilibrium; $P R^{\text {final }>1}$ denotes "final type replacement" and $P R^{\text {final }}<1$ an overall beneficial effect in the long run.

Short-term type replacement - We evaluated type replacement in the short term using $P R(a, t)$, which compares the NVT prevalence among $a$-year-old (vaccinated and unvaccinated) women after $t$ years of vaccination to the prevalence of the same age group in the pre-vaccination equilibrium.

Vaccine effectiveness - Another short-term measure for type replacement was vaccine effectiveness (VE) against NVT infection, here defined as the reduction in the risk of NVT infection in vaccinated relative to unvaccinated individuals, i.e. $V E_{n v t}(a, t)=1-R R(a, t)$, where $R R(a, t)$ denotes the corresponding relative risk evaluated among $a$-year-old women after $t$ years of vaccination. Positive VE, meaning less NVT infection among vaccinated than unvaccinated individuals, is commonly interpreted as evidence for a low risk of type replacement and negative VE as a sign of type replacement.

To scrutinise the ability of the two short-term measures to indicate final type replacement, we checked whether $\operatorname{PR}(a, t)>1$ and $V E_{n v t}(a, t)<0$ correctly indicate $P R^{\text {final }}>1$ for various choices of $a$ and $t$.

\section{Results}

\section{Convergence to the post-vaccination equilibrium}

We simulated scenarios of different levels of cross-immunity-mediated competition $(h=1,1.33, \ldots, 3)$ and vaccine-induced cross-protection $\left(\theta_{n v t}=0,10, \ldots, 50 \%\right)$. 

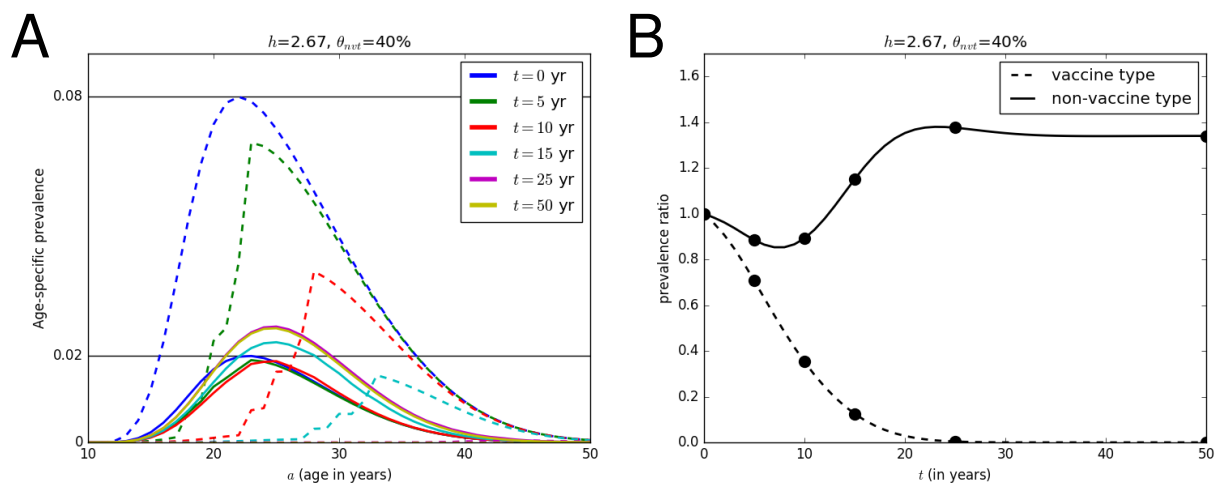

Figure 6.1: A) Age-specific prevalence of vaccine-type (dashed) and non-vaccine-type (solid) infection in women in the years following the introduction of vaccination. B) The prevalence ratio comparing the non-age-specific prevalence of all women to the prevaccination level. In both $\mathrm{A}$ and $\mathrm{B}$, the competition parameter was set to $h=2.67$ and the level of cross-protection to $\theta_{n v t}=40 \%$. In A, the black horizontal lines indicate the prevaccination maximal age-specific prevalence. Time points evaluated in $A$ are emphasised in $\mathrm{B}$ by black circles.

Throughout all scenarios in the base-case analysis, the VT was eliminated within 30 to 50 years due to the high vaccination coverage we assumed. By then, the NVT had also stabilised at the post-vaccination equilibrium Figure 6.1, Figures S6.12-14).

\section{Final type replacement is a trade-off between competition and vac- cine cross-protection}

In the absence of both vaccine-induced cross-protection $\left(\theta_{n v t}=0 \%\right)$ and competition $(h=1)$, the NVT prevalence remained unchanged (lower left corner of Figure 6.2. When increasing the strength of competition in the absence of crossprotection, the extent of type replacement in the post-vaccination equilibrium increased (bottom row in Figure 6.2). For a given level of competition $h>1$, increasing the level of vaccine-induced cross-protection attenuated the extent of final type replacement (follow each column upwards in Figure 6.2). Type replacement could even be prevented if cross-protection were strong enough. Hence, the occurrence and extent of type replacement in the post-vaccination equilibrium depend on a trade-off between the levels of competition and cross-protection. 


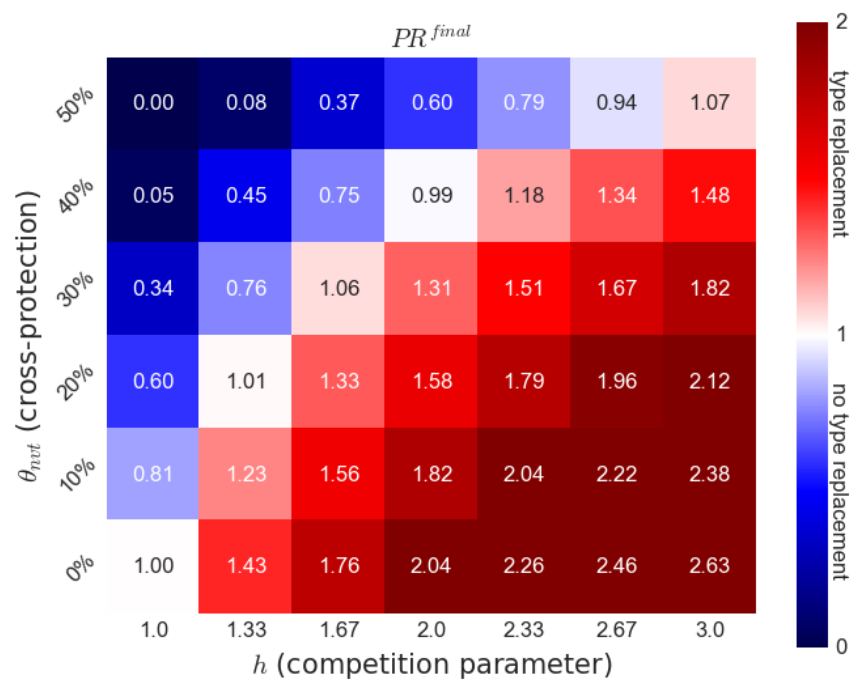

Figure 6.2: Outcomes regarding final type replacement expressed by prevalence ratio $P R^{\text {final }}$, which compares the prevalence of the non-vaccine type among all women between the pre- and post-vaccination equilibria, for different levels of competition $h$ (x-axis) and cross-protection $\theta_{n v t}$ (y-axis). Red corresponds to the occurrence of final type replacement and blue to an overall beneficial effect.

\section{Inferring final type replacement using short-term trends of non-vaccine- type prevalence}

In absence of cross-protection, short-term type replacement given by prevalence ratio $P R(a, t)$ correctly indicated final type replacement among all ages and time points (see Figure 6.3A and Figure S6.24 and compare the colours of bottom rows of Figure 6.2 and Figure 6.4A). In presence of cross-protection, short-term type replacement is also a trade-off between competition and cross-protection (see Figure 6.4A for $P R(a=20, t=10))$. However, it did not always correctly indicate the occurrence of final type replacement (compare the colours in Figure 6.2 and Figure 6.4A). Although final type replacement always occurred whenever short-term type replacement was observed, final type replacement sometimes occurred in absence of short-term type replacement. In the disagreeing scenarios, the NVT prevalence first decreased before rebounding into type replacement. In the performed simulation scenarios, such initial decreases lasted up to 20 years Figure 6.3B, Figure S6.25).

Evaluation of short-term type replacement among older women corresponded better with final type replacement but was still not perfect (compare Figure 6.2 and (Figure 6.4 $\mathrm{P})$. In addition, due to cross-protection, the NVT prevalence may undergo an age shift, whereby the NVT prevalence decreases in younger ages in 

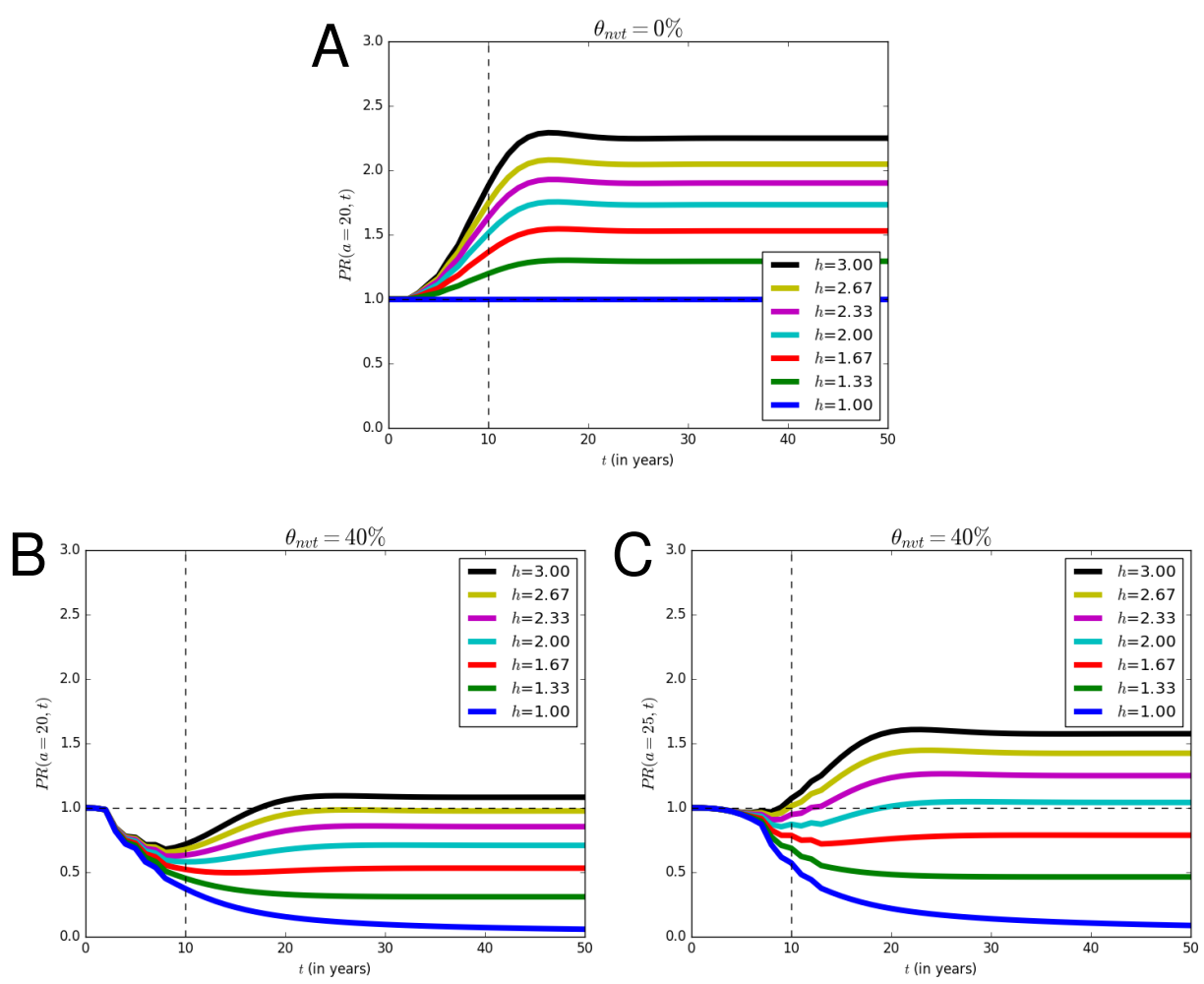

Figure 6.3: Trends of the non-vaccine-type prevalence expressed by prevalence ratio $P R(a, t)$, comparing the non-vaccine-type prevalence among 20-year-old in $(\mathrm{A}, \mathrm{B})$ and 25year-old women in $(C)$ between pre-vaccination equilibrium and $t$ years after vaccination, for different levels of competition $h$. The level of vaccine-induced cross-protection $\theta_{n v t}$ is $0 \%$ in $(A)$ and $40 \%$ in $(B, C)$. Different colours correspond to different levels of competition $(h=1,1.33, \ldots, 3)$.

the post-vaccination equilibrium but increases in older ages (Figure 6.1 A). For instance, in the scenario with $\theta_{n v t}=40 \%$ and $h=2.67$, where final type replacement did occur (Figure 6.2), the NVT prevalence among 20-year-old women stayed below the pre-vaccination level over all time $t$ after vaccination (Figure 6.33), but increased after 8 years among 25-year-old women (Figure 6.3 $\mathrm{C}$ ). Simulations showed that such sustained decreases in NVT prevalence occurred up to age 24 years in scenarios with final type replacement (Figure S6.14). Due to such age shifts, evaluating type replacement in younger age groups may fail to detect final type replacement. 

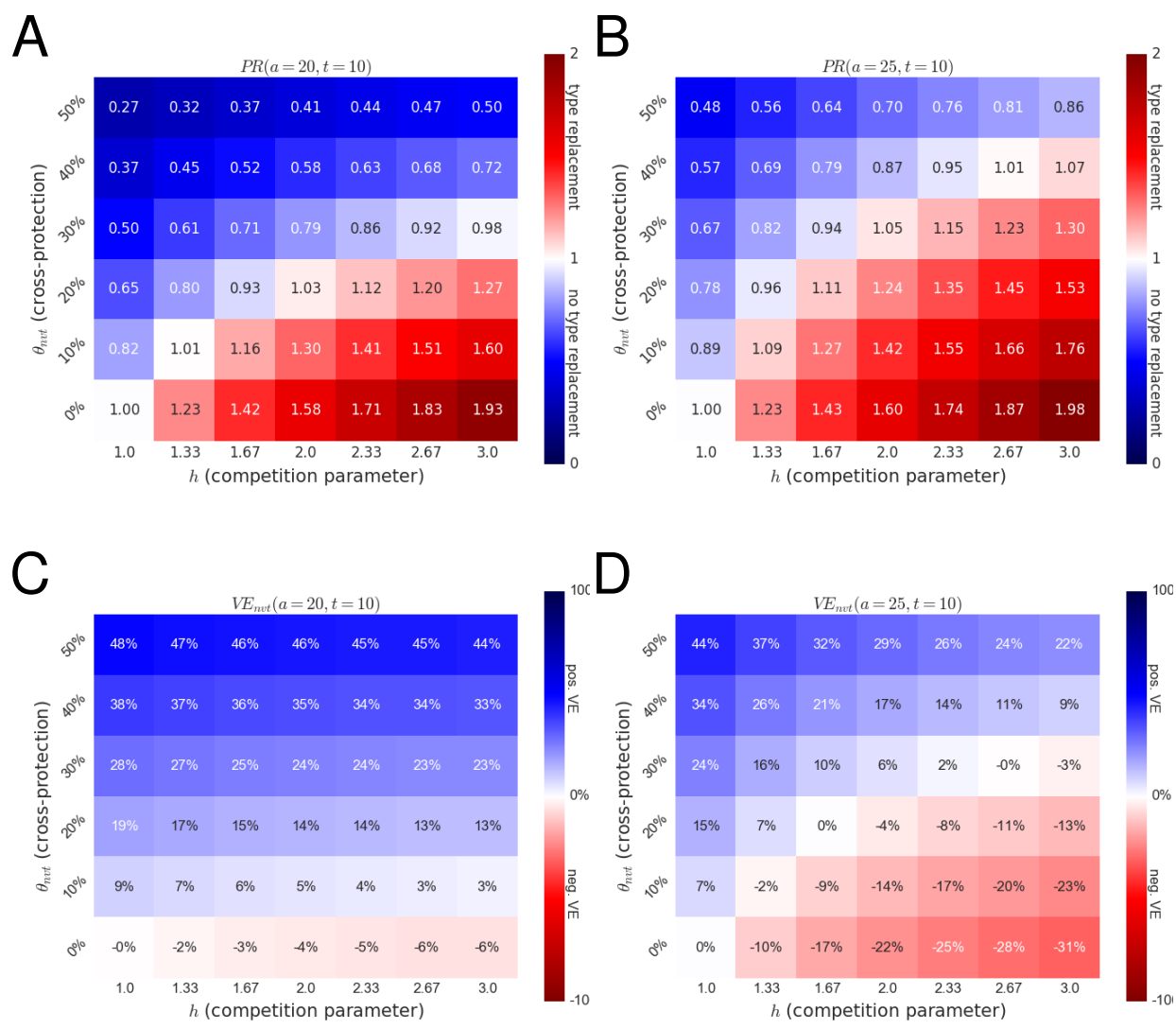

Figure 6.4: Short-term evaluation of type replacement based on prevalence ratio $P R(a, t=$ $10)$ or vaccine effectiveness $V E_{n v t}(a, t=10)$ evaluated at 10 years after vaccination. In $(\mathrm{A})$ and $(\mathrm{B}), \operatorname{PR}(a=20, t=10)$ and $P R(a=25, t=10)$ compare the prevalence of non-vaccine-type infection among 20- and 25-year-old women, respectively, between pre-vaccination equilibrium and $t=10$ years after vaccination. In (C) and (D), $V E_{n v t}(a=20, t=10)$ and $V E_{n v t}(a=25, t=10)$ compare the risk of non-vaccinetype infection among 20- and 25-year-old women, respectively, between vaccinated and unvaccinated individuals at $t=10$ years after vaccination. Red corresponds to indication of type replacement and blue to no indication based on the applied measure.

\section{Inferring final type replacement using vaccine effectiveness for the non-vaccine type}

In most simulations, vaccine effectiveness (VE), which compares the risk of NVT infection in vaccinated and unvaccinated individuals, performed poorly as an indicator of final type replacement. VE evaluated among 20-year-old women at 10 years after vaccination, $V E(a=20, t=10)$, did not discern final type replace- 
ment except in the scenarios without cross-protection (compare Figure 6.2 and Figure 6.4C). Because of the cross-protection, the risk of NVT infection in vaccinated individuals may drop below the corresponding risk in unvaccinated individuals, whereas the risk in vaccinated as well as unvaccinated individuals may have increased relative to the pre-vaccination level. Hence, positive VE for NVT infection does not rule out type replacement.

Interestingly, VE evaluated among older age groups appeared to be more sensitive for detecting final type replacement. For example, VE evaluated after 10 years among the catch-up cohort vaccinated at age 25 years corresponded better with final type replacement (compare Figure 6.2 and Figure 6.4D). However, VE at any age lost its sensitivity for detecting type replacement as time progresses; with the disappearance of the VT, vaccinated and unvaccinated individuals became more similar with respect to the amount of naturally acquired cross-immunity, after which the difference in the risks for NVT infection between the two groups became dependent on cross-protection alone (Figure S6.32). As a result, VE became immaterial for predicting type replacement.

\section{Sensitivity analyses}

In the sensitivity analyses, we encountered the same qualitative results: initial decreases in NVT prevalence before rebounding into final type replacement; occurrence of final type replacement despite positive VE; sustained decreases in NVT prevalence in young age groups (Figure S6.26-31). While retaining similar qualitative behaviour, the timing of type replacement varied among different analyses. Assuming a shorter natural immunity or a higher vaccination coverage accelerated type replacement. A lower coverage, as achieved in practice, delayed type replacement. Of interest, increasing the vaccination coverage beyond the critical level required for vaccine-type elimination appeared to reduce the scope of final type replacement by bolstering cross-protection to the NVT (compare Figure 6.2 and Figure S6.31).

\section{Discussion}

In this paper, we studied the potential emergence of genotype replacement following HPV vaccination using a transmission model in which two HPV types compete through naturally acquired, i.e. infection-induced, cross-immunity. We showed that the occurrence and timing of type replacement result from a trade-off between the levels of natural cross-immunity and vaccine-induced cross-protection. We found that cross-protection could attenuate or, if strong enough, prevent the occurrence of type replacement, in line with previous modelling studies [28, 29]. However, we discovered that with weak cross-protection, the non-vaccine-type 
prevalence may first decrease, typically for 5 to 20 years in the model, before rebounding into type replacement. Hence, 10 years follow-up since the introduction of HPV vaccination may still be too short to detect type replacement.

We are the first to suggest the existence of a "type-replacement-related" honeymoon period. This phenomenon is analogous to the general concept of a honeymoon period following vaccination, whereby the number of people who are susceptible to an infectious agent targeted by vaccination first decreases, due to the combined impact of vaccine protection and pre-existing natural immunity, but rebounds later [30-32]. In the case of a type-replacement-related honeymoon, the roles of vaccine protection and natural immunity are taken by cross-protection and natural cross-immunity, respectively. The initial decrease in the prevalence of a cross-protected type stems from the immediate cross-protection individuals receive from the vaccine, while the delayed occurrence of type replacement follows from a gradual loss of natural cross-immunity. Once the individuals who lack cross-immunity then acquire non-vaccine-type infections, the infections would last longer, in turn amplifying the transmission of the non-vaccine type, triggering type replacement.

Detection of type replacement in post-vaccination surveillance is complicated by several other factors as demonstrated in our modelled evaluations. First, vaccineinduced cross-protection may lead to a lower risk of non-vaccine-type infection in vaccinated individuals than in unvaccinated individuals, while the prevalence may increase in the general population. In other words, positive vaccine effectiveness for non-vaccine types does not necessarily preclude type replacement. As the levels of cross-protection differ by vaccine, such effects may also differ by vaccine. Second, the extent of type replacement may differ across age groups. In particular, evaluation of type replacement in young age groups may underestimate the risk in the general population. Third, the timing of type replacement depends on the vaccination coverage. With limited vaccination uptake, it may take longer to detect possible type replacement. Conversely, with ample vaccination uptake in one or both sexes, the vaccine type would be cleared more rapidly, so that potential type replacement could be detected more timely.

In addition, our analysis highlights several less-addressed insights regarding type replacement. First, increasing vaccination coverage beyond the critical threshold, required for vaccine-type elimination, still increases the benefit of cross-protection for a non-vaccine type, while the extent of competitive release for that type is already saturated. The additional amount of cross-protection in the population may suppress or even prevent type replacement, which may not have been possible at lower coverage. Second, our results suggest that those non-vaccine types for which vaccine-induced cross-protection is weak in comparison to the strength of competition bear the highest risk for type replacement. The strength of natural immunity for HPV is generally believed to be weak [25, 33], although exact estimates are hard to obtain, partly because identification of indi- 
viduals with pre-existing immunity through serological measurements is complicated [19]. The tractability of vaccination status makes estimation of the level of cross-protection more permissible. In particular, type-specific efficacies for HPV 31,33 and 45 have been estimated to be above $50 \%$ for the bivalent vaccine. A recent study suggests that the level of cross-protection might correlate with the phylogenetic distance from vaccine types [34]. Arguably, the level of natural crossimmunity follows a similar relation, meaning that closely related types would experience stronger competition and that occurrence of type replacement depends on an intricate balance with the level of vaccine-induced cross-protection. Consequently, while hypothetical, closely related HPV types against which the vaccine in use fails to evoke strong enough cross-protection are at increased risk for type replacement. The post-vaccination increases of HPV 39 and 51 in the Finnish community-randomised trial are interesting from this perspective, as these types have an intermediate phylogenetic distance to HPV 16 and 18 relative to other high-risk types.

Eventually, the potential of type replacement has implications for decisions about the prevention of HPV-related diseases. If type replacement poses a real threat, its extent might be mitigated by increasing vaccination coverage or improving vaccine effectiveness against the replacing genotypes. As different countries currently utilise different vaccines and achieve different levels of vaccination coverage, the relative merit of different vaccination strategies may also differ. In addition, there are implications for national cervical screening programmes, especially in the era of HPV DNA testing for detecting high-grade cervical lesions. As the current HPV vaccines target the most oncogenic high-risk types, the residual cancer risk coming from the non-targeted types will determine the benefit of continued screening in vaccinated women [35]. Even if vaccination triggers type replacement, the benefit of eliminating the vaccine types would probably still surpass the disease burden caused by the less-carcinogenic replacing types. However, subtle increases in the occurrence of these non-targeted types could result in considerably greater risks than anticipated based on current genotype attribution to cervical lesions and cancer [36]. Hence, disregarding type replacement may possibly lead to over-optimistic down-scaling of cervical screening programmes.

Our analysis has several strengths and weaknesses. We parametrised our model realistically based on surveys on sexual behaviour and real-life vaccination schemes [24 37]. These components are essential for generating realistic timescales in which vaccination effects propagate between cohorts. Nevertheless, our results are not meant for predicting actual levels of type-replacement or HPV trends due to simplifying model assumptions, e.g. regarding heterogeneity in sexual behaviour and vaccination uptake across risk groups, as well as uncertainty in some poorly identified parameters, especially those concerning HPV transmissibility and immunity. For instance, we assumed a relatively short duration of natural immunity of on average 1 and 10 years in men and women, respectively, 
while other modelling studies tend to assume longer immunity [23]. Our sensitivity analyses showed that longer-lasting natural immunity leads to prolonged honeymoon periods, further complicating the detection of type replacement from early post-vaccination surveillance. The assumed asymmetry in the duration of natural immunity in men and women also differs from some other modelling studies, but we expect the duration of the honeymoon period to be mainly driven by the sex with the longer-lasting immunity. This remains to be determined. In addition, the risk of type replacement may be underestimated if cross-protection to non-vaccine types lasts considerably shorter than vaccine-type protection.

Finally, the possibility of type replacement should not discourage efforts of further enhancing HPV vaccination programmes worldwide. Our analysis shows that the risk of type replacement may generally be diminished by increasing vaccination coverage and by providing improved effectiveness against possibly replacing oncogenic HPV types. These arguments plead in favour of gender-neutral HPV vaccination with broad-spectrum vaccines, adding another dimension to the construction of resilient vaccination programmes [38]. Nevertheless, monitoring type replacement remains important, especially among HPV types and age groups that bear the highest risks for type replacement. Ultimately, improved assessment of type replacement may help to further optimise HPV control measures and to eliminate cervical cancer as a public health problem.

\section{References}

[1] E.-M. de Villiers. "Cross-roads in the classification of papillomaviruses". Virology 445.1-2 (2013), 2-10.

[2] R. B. Roden and P. L. Stern. "Opportunities and challenges for human papillomavirus vaccination in cancer". Nat Rev Cancer 18.4 (2018), 240-254.

[3] M. Plummer, C. de Martel, J. Vignat, et al. "Global burden of cancers attributable to infections in 2012: a synthetic analysis". Lancet Glob Health 4.9 (2016), e609-e616.

[4] W. H. Organization. WHO Director-General calls for all countries to take action to help end the suffering caused by cervical caner. Accessed: 2018-05-19. URL: http://www . who . int/ reproductivehealth/call-to-action-eliminationcervical-cancer/en/

[5] M. Lehtinen and J. Dillner. "Clinical trials of human papillomavirus vaccines and beyond". Nat Rev Clin Oncol 10.7 (2013), 400-410.

[6] E. A. Joura, A. R. Giuliano, O.-E. Iversen, et al. "A 9-valent HPV vaccine against infection and intraepithelial neoplasia in women". N Engl J Med 372.8 (2015), 711-723.

[7] D. Mesher, K. Soldan, M. Lehtinen, et al. "Population-level effects of human papillomavirus vaccination programs on infections with nonvaccine genotypes". Emerg Infect Dis 22.10 (2016), 1732-1740.

[8] M. Drolet, É. Bénard, N. Pérez, et al. "Population-level impact and herd effects following the introduction of human papillomavirus vaccination programmes: updated systematic review and meta-analysis". Lancet 394.10197 (2019), 497-509. 
[9] M. Lehtinen, T. Luostarinen, S. Vänskä, et al. "Gender-neutral vaccination provides improved control of human papillomavirus types 18/31/33/35 through herd immunity: Results of a community randomized trial (III)". Int J Cancer 143.9 (2018), 2299-2310.

[10] P. J. Woestenberg, A. J. King, B. H. Van Benthem, et al. "Bivalent vaccine effectiveness against type-specific HPV positivity: evidence for cross-protection against oncogenic types among Dutch STI clinic visitors". J Infect Dis 217.2 (2018), 213-222.

[11] M. Stanley and L. L. Villa. "Monitoring HPV vaccination". Vaccine 26 (2008), A24-A27.

[12] J. O. Lloyd-Smith. "Vacated niches, competitive release and the community ecology of pathogen eradication". Philos Trans R Soc B Biol Sci 368.1623 (2013), 20120150.

[13] D. M. Weinberger, R. Malley, and M. Lipsitch. "Serotype replacement in disease after pneumococcal vaccination". Lancet 378.9807 (2011), 1962-1973.

[14] S. Gandon and T. Day. "Evidences of parasite evolution after vaccination". Vaccine 26 (2008), C4-C7.

[15] M. Lehtinen and J. Paavonen. "Vaccination against human papillomaviruses shows great promise". Lancet 364.9447 (2004), 1731-1732.

[16] L. F. Xi, Z. R. Edelstein, C. Meyers, et al. "Human papillomavirus types 16 and 18 DNA load in relation to coexistence of other types, particularly those in the same species". Cancer Epidemiol Biomarkers Prev 18.9 (2009), 2507-2512.

[17] J. Biryukov and C. Meyers. "Superinfection exclusion between two high-risk human papillomavirus types during a coinfection". J Virol 92.8 (2018), e01993-17.

[18] J. E. Tota, M. Jiang, A. V. Ramanakumar, et al. "Epidemiologic evaluation of human papillomavirus type competition and the potential for type replacement post-vaccination". PLoS One 11.12 (2016), e0166329.

[19] D. P. Durham, E. M. Poolman, Y. Ibuka, et al. "Reevaluation of epidemiological data demonstrates that it is consistent with cross-immunity among human papillomavirus types". J Infect Dis 206.8 (2012), 1291-1298.

[20] I. Man, J. Wallinga, and J. A. Bogaards. "Inferring pathogen type interactions using crosssectional prevalence data: opportunities and pitfalls for predicting type replacement". Epidemiology 29.5 (2018), 666-674.

[21] J. E. Tota, F. Struyf, M. Merikukka, et al. "Evaluation of type replacement following HPV16/18 vaccination: pooled analysis of two randomized trials". J Natl Cancer Inst 109.7 (2017), djw300.

[22] P. Gray, J. Palmroth, T. Luostarinen, et al. "Evaluation of HPV type-replacement in unvaccinated and vaccinated adolescent females - Post-hoc analysis of a community-randomized clinical trial (II)". Int J Cancer 142.12 (2018), 2491-2500.

[23] M. Brisson, É. Bénard, M. Drolet, et al. "Population-level impact, herd immunity, and elimination after human papillomavirus vaccination: a systematic review and meta-analysis of predictions from transmission-dynamic models". Lancet Public Health 1.1 (2016), e8-e17.

[24] H. De Graaf and C. Wijsen. Seksuele gezondheid in Nederland 2017. 2017. URL: https : //www .rutgers.nl/feiten-en-cijfers/seksuele-gezondheid-en-gedrag/seksuelegezondheid-nederland-2017.

[25] S. L. Ranjeva, E. B. Baskerville, V. Dukic, et al. "Recurring infection with ecologically distinct HPV types can explain high prevalence and diversity”. Proc Natl Acad Sci U S A 114.51 (2017), 13573-13578.

[26] A. R. Giuliano, A. G. Nyitray, A. R. Kreimer, et al. "EUROGIN 2014 roadmap: Differences in HPV infection natural history, transmission, and HPV-related cancer incidence by gender and anatomic site of infection". Int J Cancer 136.12 (2015), 2752-2760. 
[27] V. M. H. Coupé, J. Berkhof, N. W. J. Bulkmans, et al. "Age-dependent prevalence of 14 highrisk HPV types in the Netherlands: implications for prophylactic vaccination and screening". $\mathrm{Br}$ J Cancer 98.3 (2008), 646-651.

[28] E. M. Poolman, E. H. Elbasha, and A. P. Galvani. "Vaccination and the evolutionary ecology of human papillomavirus". Vaccine 26 (2008), C25-C30.

[29] C. L. Murall, C. T. Bauch, and T. Day. "Could the human papillomavirus vaccines drive virulence evolution?" Proc R Soc 282.1798 (2015), 20141069.

[30] M. Brisson and W. J. Edmunds. "Economic evaluation of vaccination programs: the impact of herd-immunity". Med Decis Making 23.1 (2003), 76-82.

[31] S. Gandon and T. Day. "The evolutionary epidemiology of vaccination". J R Soc Interface 4.16 (2007), 803-817.

[32] A. R. Mclean. "Vaccination, evolution and changes in the efficacy of vaccines: a theoretical framework". Proc Biol Sci 261.1362 (1995), 389-393.

[33] K. K. Thomas, J. P. Hughes, J. M. Kuypers, et al. "Concurrent and sequential acquisition of different genital human papillomavirus types". J Infect Dis 182.4 (2000), 1097-1102.

[34] J. A. Bogaards, P. Van Der Weele, P. J. Woestenberg, et al. "Bivalent human papillomavirus (HPV) vaccine effectiveness correlates with phylogenetic distance from HPV vaccine types 16 and 18". J Infect Dis 220.7 (2019), 1141-1146.

[35] K. Cuschieri, G. Ronco, A. Lorincz, et al. "Eurogin roadmap 2017: triage strategies for the management of HPV-positive women in cervical screening programs". Int J Cancer 143.4 (2018), 735-745.

[36] B. I. Lissenberg-Witte, J. A. Bogaards, W. G. Quint, et al. "Estimating the human papillomavirus genotype attribution in screen-detected high-grade cervical lesions". Epidemiology 30.4 (2019), 590-596.

[37] J. M. L. Brotherton and P. N. Bloem. "Population-based HPV vaccination programmes are safe and effective: 2017 update and the impetus for achieving better global coverage". Best Pract Res Clin Obstet Gynaecol 47 (2018), 42-58.

[38] K. M. Elfström, F. Lazzarato, S. Franceschi, et al. "Human papillomavirus vaccination of boys and extended catch-up vaccination: effects on the resilience of programs". J Infect Dis 213.2 (2016), 199-205.

[39] S. Vänskä, K. Auranen, T. Leino, et al. "Impact of vaccination on 14 high-risk HPV type infections: a mathematical modelling approach". PLoS One 8.8 (2013), e72088.

[40] A. De Roos, O. Diekmann, and J. Metz. "Studying the dynamics of structured population models: a versatile technique and its application to Daphnia". Am Nat 139 (1992), 123-147.

[41] H. De Graaf, M. Van Den Borne, S. Nikkelen, et al. Seksuele gezondheid van jongeren in Nederland anno 2017. 2017. URL: https://www.rutgers.nl/seks-onder-je-25e-2017

[42] K. Kavanagh, K. G. Pollock, K. Cuschieri, et al. "Changes in the prevalence of human papillomavirus following a national bivalent human papillomavirus vaccination programme in Scotland: a 7-year cross-sectional study". Lancet Infect Dis 17.12 (2017), 1293-1302.

[43] E. van Lier, J. Geraedts, P. Oomen, et al. Vaccinatiegraad en jaarverslag Rijksvaccinatieprogramma Nederland 2017. 2018. URL:https://www.rivm.nl/publicaties/vaccinatiegraaden-jaarverslag-rijksvaccinatieprogramma-nederland-2017

[44] National HPV Vaccination Program Register of Australia. 2018. URL: http://www . hpvregister. org.au/research/coverage-data 


\section{Appendices of Chapter 6}

\section{A. Transmission model}

\section{A.1. Demographics}

We describe a transmission model of two competing HPV types in an open, heterosexual population of age between $a_{\text {enter }}=10$ and $a_{\text {exit }}=70$ years. We assume a constant rate of individuals entering the population at age $a_{\text {enter }}$. The number of individuals newly entering the population is $m$ per year, which is set to unity to ease computation, without loss of generality. Throughout all birth cohorts, the proportions of male and female are $\left\{p_{m}, p_{f}\right\}=\left\{\frac{1}{2}, \frac{1}{2}\right\}$, respectively. All individuals leave the population at age 70 years exactly, and there is no exit due to death before this age.

The population is further stratified into a low-risk and a high-risk group with proportions $\left\{p_{l}, p_{h}\right\}=$ $\left\{\frac{4}{5}, \frac{1}{5}\right\}$, respectively. We assume each individual to stay in the same risk group throughout his/her lifetime. Individuals of the same age but from different risk groups differ in their contact rates, with the rate of the high-risk group being higher than the rate of the low-risk group.

In addition, the population is stratified into vaccinated and unvaccinated individuals. Within each sex, vaccinated individuals from the same birth cohorts are all vaccinated at the same age, which can be an age later than $a_{\text {enter }}$. Coverage of vaccination depends on time and sex but not on the risk-group stratification.

To denote the sets of strata in the population, we introduce the following notations:

- $\mathcal{A}=\left[a_{\text {enter }}, a_{\text {exit }}\right]$ : age range for the sexually active population;

- $\mathcal{G}=\{f, m\}$ : female and male;

- $\mathcal{R}=\{l, h\}$ : low-risk and high-risk;

- $\mathcal{W}=\{u, v\}$ : unvaccinated and vaccinated.

See Figure S6.1 for the division of a given birth cohort of age $a$ into smaller strata.

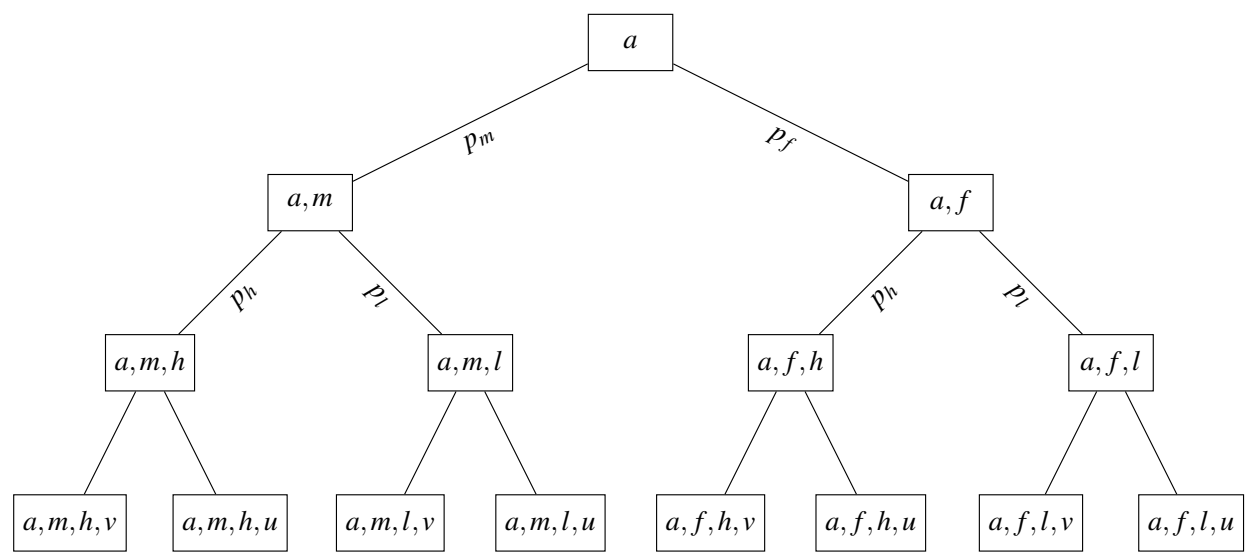

Figure S6.1: Stratification of individuals aged $a$ into strata according to sex, risk group, and vaccination status. 


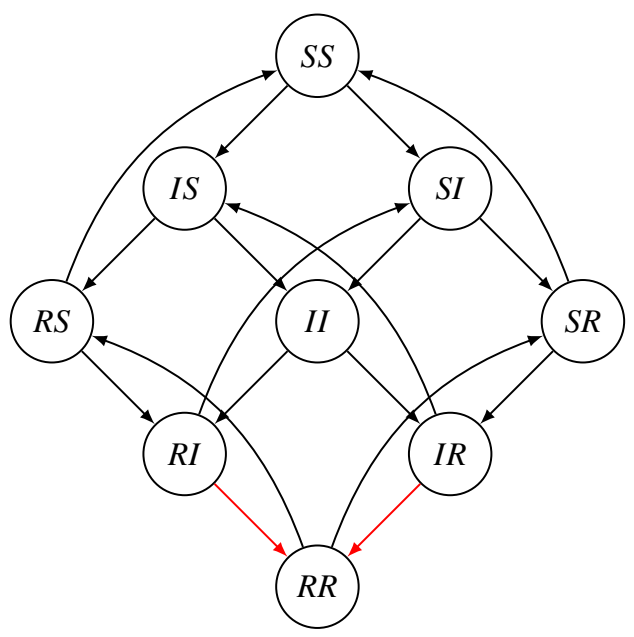

Figure S6.2: The nine infection states and the respective transitions in the two-type susceptibleifected-recovered-susceptible model. The arrows correspond to transitions of acquisition, clearance, and waning of immunity. In red are the transitions of clearance that are accelerated by a factor of $h$ due to competition through naturally acquired, i.e. infection-induced, cross-immunity.

\section{A.2. Infection states}

The model prescribes the transmission dynamics of two competing HPV types, one vaccine type $(v t)$ and one non-vaccine type $(n v t)$. Both types follow susceptible-infected-recovered-susceptible (SIRS) dynamics. Hence, there are nine infection states in total, each characterised by a pairwise combination of $S, I$ and $R$ (Figure S6.2): $\mathcal{S}=\{S S, I S, S I, I I, R S, S R, R I, I R, R R\}$. The $i$-th letter of a pairwise combination indicates the status with respect to type $i$. Acquisition and clearance of infection are assumed to occur sequentially so that only transitions between infection states differing with respect to the status of only one type are possible [Figure S6.2.

Each individual enters the sexual mixing population being susceptible to both types $(S S)$. Subsequently, he/she transits between infection states due to acquisition, clearance, and waning of natural immunity with respect to either of the two types until age 70 years.

To denote the number of individuals in infection state $X$ within a given stratum $(a, g, r, w)$, we use $X(a, g, r, w)$, where $X \in \mathcal{S}, a \in \mathcal{A}, g \in \mathcal{G}, r \in \mathcal{R}$ and $w \in \mathcal{W}$.

\section{A.3. Sexual contact patterns}

Patterns of sexual contact depend on sex, age, and risk stratification but not on vaccination status. Firstly, we allow only heterosexual contact. Secondly, contacts between individuals of different ages and risk groups are assortative such that contacts between similar ages and within the same risk group are preferred. In the following, we first specify the per capita contact rates of individuals in different strata in Section A.3.1. In Section A.3.2, we then specify the mixing distribution, i.e. how the contact of an individual of any stratum is distributed among other strata. The contact pattern we use here is a simplified version of the contact pattern suggested by Vänskä et al. [39].

\section{A.3.1. Per capita contact rate}


The mean per capita contact rate of different ages $\bar{c}(a)$ is given by the following equation (the black line in Figure S6.3A):

$$
\bar{c}(a)= \begin{cases}\left(\frac{\bar{c}_{\max }}{\tau}\right)\left(a-a_{\text {enter }}\right)^{\kappa} \exp ^{\left(-\frac{a-a_{\text {enter }}}{\omega}\right)} & , \text { for } a \in\left[a_{\text {enter }}, a_{\text {exit }}\right], \\ 0 & , \text { otherwise. }\end{cases}
$$

The parameters in the above expression are

- $a_{\text {enter }}$ : age of entering sexual mixing population, as introduced earlier;

- $a_{\bar{c}_{\max }}:$ age at which the maximum mean contact rate is attained;

- $\bar{c}_{\text {max }}$ : maximum mean contact rate among all ages;

- $\omega$ : width of decrease in mean contact rate when deviating from age $a_{\bar{c}_{\text {max }}}$;

- $\kappa=\left(a_{\bar{c}_{\max }}-a_{\text {enter }}\right) / \omega$;

- $\tau=(\kappa \omega)^{\kappa} \exp ^{(-\kappa)}$.

Note that, by construction, it follows that $\bar{c}_{\max }=\max _{a} \bar{c}(a)$ and $a_{\bar{c}_{\max }}=\underset{a}{\operatorname{argmax}} \bar{c}(a)$.

From the mean contact rate $\bar{c}(a)$, we then derive the risk-group-specific contact rates, $c(a, l)$ and $c(a, h)$ by solving the following system of equations:

$$
\begin{cases}\bar{c}(a)= & p_{l} c(a, l)+p_{h} c(a, h) \\ \left(c_{v} \cdot \bar{c}(a)\right)^{2}= & p_{l}(c(a, l)-\bar{c}(a))^{2}+p_{h}(c(a, h)-\bar{c}(a))^{2} .\end{cases}
$$

The terms in the above equations are

- $c_{v}$ : coefficient of variance of contact rate among risk groups;

- $p_{l}, p_{h}$ : proportions of individuals in the low- and high-risk group, respectively, as introduced earlier.

Solving this system of equations yields the following expressions for $c(a, l)$ and $c(a, h)$ Figure S6.3A):

$$
\begin{aligned}
c(a, l) & =\bar{c}(a)\left(1-c_{v} \sqrt{p_{h} / p_{l}}\right), \\
c(a, h) & =\bar{c}(a)\left(1+c_{v} \sqrt{p_{l} / p_{h}}\right) .
\end{aligned}
$$

\section{A.3.2. Sexual contact mixing distribution}

The mixing distribution of sexual contact between individuals of different strata is constructed in the following three steps:

- constructing the mixing distribution of contact between risk groups;

- constructing the mixing distribution of contact between age groups;

- combining the two mixing distributions.

In the first step, the mixing distribution between risk groups $Q_{\mathcal{R}}=\left(\begin{array}{cc}q_{\mathcal{R}}(l, h) & q_{\mathcal{R}}(l, h) \\ q_{\mathcal{R}}(h, l) & q_{\mathcal{R}}(h, h)\end{array}\right)$ is computed according to the following equations:

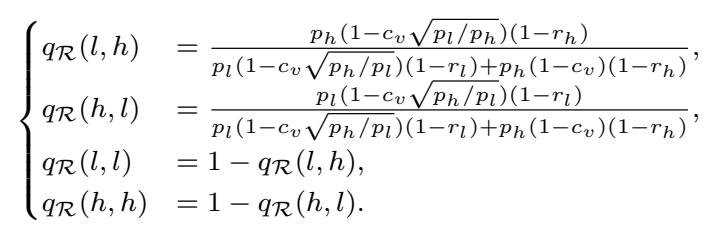

The terms in the above equations are 

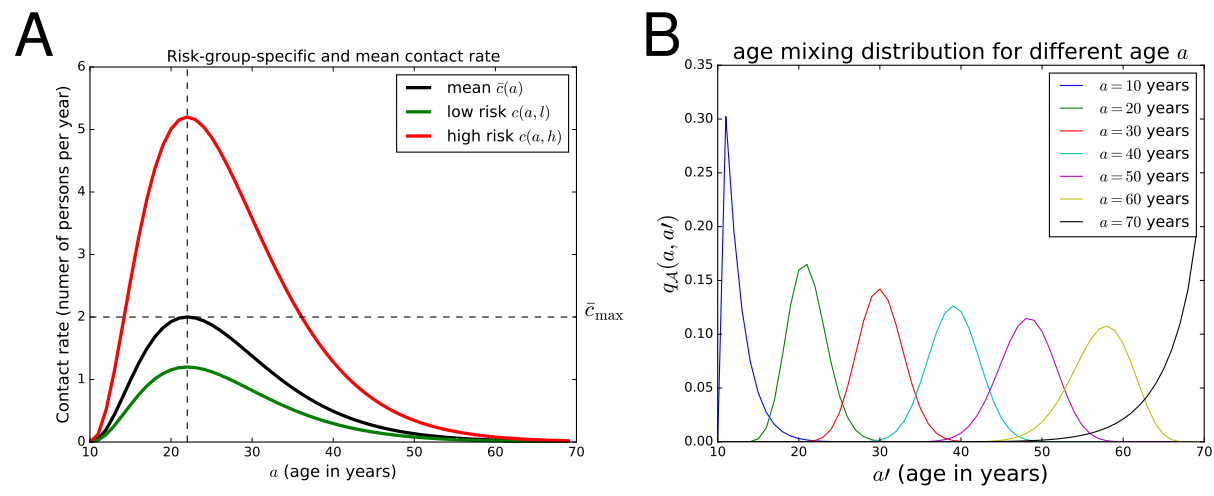

Figure S6.3: A) Per capita rates of sexual contact of the high-risk group $c(a, h)$ in red, the low-risk group $c(a, l)$ in green, and their mean $\bar{c}(a)$ in black. The unit of these rates is persons per year. In $\mathrm{A}$, the mean contact rate attains its maximum value $\bar{c}_{\max }$ at age $a_{\bar{c}_{\max }}$, as given by the dashed lines. Here, $\bar{c}_{\max }$ and $a_{\bar{c}_{\max }}$ are set as 2 persons per year and age 22 years, respectively. B) Mixing distribution $q_{\mathcal{A}}\left(a, a^{\prime}\right)$ of sexual contact by individuals of different age $a=10,20, \cdots, 70$ to another age $a^{\prime}$.

- $r_{l}, r_{h}$ : proportions of contacts of individuals in the low- and high-risk groups that are reserved among themselves (assortativity parameters);

- $p_{l}, p_{h}$ : proportions of individuals in the low- and high-risk groups, respectively, as introduced earlier;

- $c_{v}$ : coefficient of variance, as introduced earlier.

In the second step, the mixing distribution between age groups $q_{\mathcal{A}}\left(a, a^{\prime}\right)$ is computed according to the following beta distribution (Figure S6.33):

$$
q_{\mathcal{A}}\left(a, a^{\prime}\right)=\frac{\gamma(\alpha(a)+\beta(a)) \phi\left(a^{\prime}\right)^{\alpha(a)-1}\left(1-\phi\left(a^{\prime}\right)\right)^{\beta(a)-1}}{\gamma(\alpha(a)) \gamma(\beta(a))\left(a_{\text {exit }}-a_{\text {enter }}\right)}, \text { where }
$$

- $a_{\text {enter: }}$ : age of entering sexual mixing population, as introduced earlier;

- $a_{e x i t}$ : age of exiting sexual mixing population, as introduced earlier;

- $\phi(a)=\left(a-a_{\text {enter }}\right) /\left(a_{\text {exit }}-a_{\text {enter }}\right)$;

- $\mu(a)$ : mean age of the contacts of an individual of age $a$ as defined in the paragraph below;

- $\sigma^{2}(a)$ : variance age of the contacts of an individual of age $a$ as defined in the paragraph below;

- $\alpha(a)=\mu(a)\left(\frac{\mu(a)(1-\mu(a))}{\sigma(a)}-1\right)$;

- $\beta(a)=(1-\mu(a))\left(\frac{\mu(a)(1-\mu(a))}{\sigma(a)}-1\right)$.

The mean age of the contact by an individual of age $a, \mu(a)$, is assumed to be larger than $a$ when $a$ is small and smaller than $a$ when $a$ is large (see Figure S6.4 A). This reflects the preference of young individuals to have older partners and vice versa. We assumed $\mu(a)$ to increase linearly starting from $a_{\text {enter }}+\delta_{\max }$ at $a_{\text {enter }}$ to $a_{\text {exit }}-\delta_{\max }$ at $a_{\text {exit }}$. The corresponding variance of the age of the contact by an individual of age $a, \sigma^{2}(a)$, is also assumed to be a linearly increasing function, which equals to $\sigma_{\text {min }}^{2}$ at $a_{\text {enter }}$ to $\sigma_{\text {max }}^{2}$ at $a_{\text {exit }}$ (see Figure S6.4 3 ).

From the mixing distributions between risk groups and age groups, we then derive the whole mixing distribution, given by $c\left(a, g, r, a^{\prime}, g^{\prime}, r^{\prime}\right)$. This function describes how many contacts an individual from 

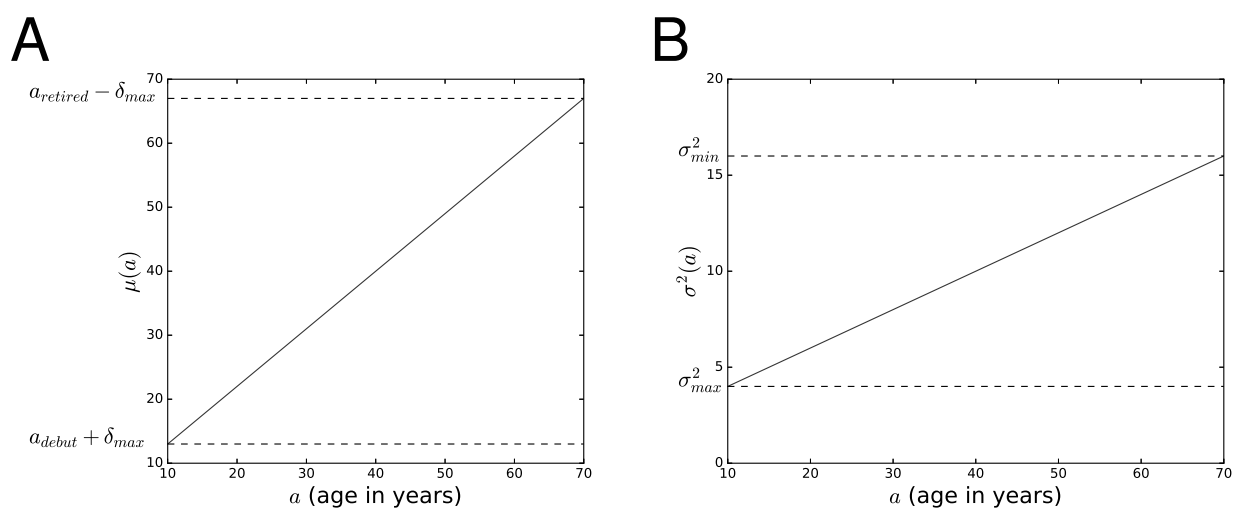

Figure S6.4: A) Mean age of the contact by individuals of different age $a, \mu(a)$. Here, $\delta_{\max }$ is set as 3 years. B) Variance of the age of the contact by individuals of different age $a, \sigma^{2}(a)$. Here, $\sigma_{m i n}^{2}$, $\sigma_{\max }^{2}$ are set as 4 and 16 years $^{2}$, respectively.

a given stratum $(a, g, r)$ has with individuals of another stratum $\left(a^{\prime}, g^{\prime}, r^{\prime}\right)$, where $a, a^{\prime} \in \mathcal{A}, g \neq g^{\prime} \in \mathcal{G}$ and $r, r^{\prime} \in \mathcal{R}$ :

$$
c\left(a, g, r, a^{\prime}, g^{\prime}, r^{\prime}\right)=\frac{p_{r} c(a, r) q_{\mathcal{A}}\left(a, a^{\prime}\right) q_{\mathcal{R}}\left(r, r^{\prime}\right)+p_{r^{\prime}} c\left(a^{\prime}, r\right) q_{\mathcal{A}}\left(a^{\prime}, a\right) q_{\mathcal{R}}\left(r^{\prime}, r\right)}{p_{r}} .
$$

The multiplication with $p_{r}$ and $p_{r^{\prime}}$ in the above equation ensures the total number of contacts between any two strata is balanced, meaning that the total number of contacts from individuals of any given stratum to individuals of another stratum is exactly equal to the total number of contact vice versa. Note that due to this balance correction, the resulting value of $c\left(a, g, r, a^{\prime}, g^{\prime}, r^{\prime}\right)$ may slightly differ from the product $c(a, r) q_{\mathcal{A}}\left(a, a^{\prime}\right) q_{\mathcal{R}}\left(r, r^{\prime}\right)$.

\section{A.4. Transition rates}

Now the rates of sexual contact are defined, we continue with introducing the related rates of acquiring infection and the rates of clearance and waning of natural immunity. The per capita rate of any given transition is determined by one or more of the following three components:

- baseline rate;

- multiplicative factor due to between-type competition;

- multiplicative factor due to the vaccination status.

In the following subsections, we define the rates of state transitions due to acquisition, clearance and waning of natural immunity, consecutively. We use $P_{X, Y}(a, g, r, w)$ to denote the rate of an individual from stratum $(a, g, r, w)$ to move from state $X$ to state $Y$. For completeness, transition rates corresponding to impossible transitions are set to zero, e.g. $P_{S S \rightarrow R S}(a, g, r, w)=0$ Figure S6.2.

\section{A.4.1. Acquisition rates}

The type-specific baseline acquisition rate $\lambda_{i}(a, g, r)$ of stratum $(a, g, r)$ has the following expression:

$$
\lambda_{i}(a, g, r)=\beta_{i} \sum_{r^{\prime} \in \mathcal{R}} \int_{a_{\text {enter }}}^{a_{\text {exit }}} c\left(a, g, r, a^{\prime}, g^{\prime}, r^{\prime}\right) \frac{I_{i}\left(a^{\prime}, g^{\prime}, r^{\prime}\right)}{m p_{g^{\prime}} p_{r^{\prime}}} d a^{\prime} \text {, where }
$$


- $g^{\prime}$ : opposite sex;

- $m$ : birth rate, which is set to unity, i.e. $m=1$;

- $\beta_{i}$ : probability of successfully acquiring type- $i$ infection given established contact with an individual infected with type $i$, where $i \in\{v t, n v t\}$

- $I_{v t}\left(a^{\prime}, g^{\prime}, r^{\prime}\right)=\sum_{w^{\prime} \in \mathcal{W}} I S\left(a^{\prime}, g^{\prime}, r^{\prime}, w^{\prime}\right)+I I\left(a^{\prime}, g^{\prime}, r^{\prime}, w^{\prime}\right)+I R\left(a^{\prime}, g^{\prime}, r^{\prime}, w^{\prime}\right)$ : all individuals in stratum $\left(a^{\prime}, g^{\prime}, r^{\prime}\right)$ infected with the vaccine type;

- $I_{n v t}\left(a^{\prime}, g^{\prime}, r^{\prime}\right)=\sum_{w^{\prime} \in \mathcal{W}} S I\left(a^{\prime}, g^{\prime}, r^{\prime}, w^{\prime}\right)+I I\left(a^{\prime}, g^{\prime}, r^{\prime}, w^{\prime}\right)+R I\left(a^{\prime}, g^{\prime}, r^{\prime}, w^{\prime}\right)$ : all individuals in stratum $\left(a^{\prime}, g^{\prime}, r^{\prime}\right)$ infected with the non-vaccine type.

Together with the multiplicative factors, the acquisition rates of an individual belonging to stratum $(a, g, r, w)$ from different infection states are

$$
\begin{aligned}
& P_{S S \rightarrow I S}(a, g, r, w)=P_{S I \rightarrow I I}(a, g, r, w)=P_{S R \rightarrow I R}(a, g, r, w)=\lambda_{v t}(a, g, r) \cdot\left(1-\theta_{v t}\right)^{\mathbb{1}[w=v]}, \\
& P_{S S \rightarrow S I}(a, g, r, w)=P_{I S \rightarrow I I}(a, g, r, w)=P_{R S \rightarrow R I}(a, g, r, w)=\lambda_{n v t}(a, g, r) \cdot\left(1-\theta_{n v t}\right)^{\mathbb{1}[w=v] .}
\end{aligned}
$$

Here, $\theta_{i}$ denotes the vaccine efficacy for type $i$. The indicator function $\mathbb{1}[w=v]$ ensures that multiplication with $\left(1-\theta_{i}\right)$ only applies to those that are vaccinated.

\section{A.4.2. Clearance rates}

The type-specific baseline clearance rates, $\mu_{v t}$ and $\mu_{n v t}$, are constant and identical for all strata. Together with the multiplicative factors, the clearance rates from different infection states of an individual from stratum $(a, g, r, w)$ are

$$
\begin{aligned}
& P_{I S \rightarrow R S}(a, g, r, w)=\mu_{v t}, \\
& P_{I I \rightarrow R I}(a, g, r, w)=\mu_{v t}, \\
& P_{I R \rightarrow R R}(a, g, r, w)=\mu_{v t} \cdot h, \\
& P_{S I \rightarrow S R}(a, g, r, w) \mu_{n v t}, \\
& P_{I I \rightarrow I R}(a, g, r, w)=\mu_{n v t}, \\
& P_{R I \rightarrow R R}(a, g, r, w)=\mu_{n v t} \cdot h .
\end{aligned}
$$

Here, $h$ is the competition parameter governing the strength of naturally acquired, i.e. infectioninduced, cross-immunity. Note that the expressions above are all independent of the chosen stratum $(a, g, r, w)$. Hence, these rates are identical for all strata.

\section{A.4.3. Rates of waning immunity}

The rate of waning immunity depends only on the sex. It is denoted by $\gamma_{g}$, where $g \in \mathcal{G}$ :

$$
\begin{aligned}
P_{R S \rightarrow S S}(a, g, r, w) & =P_{R I \rightarrow S I}(a, g, r, w)=P_{R R \rightarrow S R}(a, g, r, w)=P_{S R \rightarrow S S}(a, g, r, w) \\
& =P_{I R \rightarrow I S}(a, g, r, w)=P_{R R \rightarrow R S}(a, g, r, w)=\gamma_{g} .
\end{aligned}
$$

\section{A.4.4. Transition matrix}

For brevity, we abbreviate the notation for strata using $\Theta=(a, g, r, w)$. Then, we summarise all possible transition rates corresponding to an individual of stratum $\Theta$ compactly in a nine-by-nine transition matrix $\Psi(\Theta)$. In this matrix, the entries corresponding to different incoming and outcoming infection states are indexed in the order of $\{S S, I S, S I, I I, R S, S R, R I, I R, R R\}$. For any pair of states $X, Y \in \mathcal{S}$, the entry of $\Psi(\Theta)$ in the row of $X$ and the column of $Y$ is defined by $P_{X \rightarrow Y}(\Theta)$, the rate of an individual of stratum $\Theta$ moving from state $X$ to $Y$.

Furthermore, the diagonal elements of the transition matrix $\Psi(\Theta)$ are defined by $(-1)$ times the sum of all other elements in the same row, i.e. the diagonal element of the row of state $X$ is equal to $-\sum_{Y \neq X} P_{X \rightarrow Y}(\Theta)$. 


\section{A.5. The system of partial differential equations}

Until now, we have suppressed the dependence of the defined quantities on time $t$ and have mentioned how they evolve in time. Here, we write down $X(\Theta, t), \lambda_{i}(\Theta, t)$ and $\Psi(\Theta, t)$ formally as quantities that change in time, as described above due to events of acquisition, clearance and waning of immunity throughout the population. With these new notations including time, we can now write down the system of partial differential equations describing how the model evolves in time. We do that in the following vector form. The equations in this system describe for each stratum how the corresponding individuals transit between state and become older as time passes by applying the transition matrix to the current situation:

$$
\frac{\partial \mathbf{X}(\Theta, t)}{\partial t}+\frac{\partial \mathbf{X}(\Theta, t)}{\partial a}=\Psi(\Theta, t)^{T} \mathbf{X}(\Theta, t),
$$

where $\mathbf{X}(\Theta, t)=[S S(\Theta, t), I S(\Theta, t), S I(\Theta, t), I I(\Theta, t), R S(\Theta, t), S R(\Theta, t), R I(\Theta, t), I R(\Theta, t), R R(\Theta, t)]^{T}$ is the state vector of a given stratum $\Theta=(a, g, r, w)$. Note that the entire system of equations consists of the above equation for all strata.

In addition, the system of equations also has a boundary condition, which governs that the influx of individuals into the population. In the model, we assume all individuals to enter unvaccinated. Hence the boundary condition for stratum $(g, r, u)$ at age $a_{\text {enter }}$, where $g \in \mathcal{G}$ and $r \in \mathcal{R}$, is:

$$
S S\left(a_{\text {enter }}, g, r, u, t\right)=m \cdot p_{g} \cdot p_{r} .
$$

To model vaccination, we just transfer a proportion of a cohort from an unvaccinated stratum into the corresponding vaccinated stratum.

\section{A.6. An approximation method for simulation}

Simulation of the system of partial differential equations, described in the previous section, is done using a numerical approximation called escalator boxcar train. This method discretises the continuous influx of individuals by lumping together individuals that are born within some pre-fixed interval. Here, this interval is set to be a year. Furthermore, we discretise $c\left(a, g, r, a^{\prime}, g^{\prime}, r^{\prime}\right)$ into a stepwise constant function with step size of one year as well. A detailed description of this approximation method is provided by de Roos et al. 40 .

\section{B. Parametrisation and calibration}

\section{B.1. Overview of parameters}

Parametrisation of the model was partly based on data from literature and partly derived from calibration. Parametrisation of the contact patterns was based on surveys of sexual behaviours in the Netherlands [Figure S6.3 and Figure S6.4. 24 41] The derived parameters values are given in Table S6.1. This table also presents the chosen values of the demographic parameters. The transmission probabilities and clearance rates were obtained by calibrating the model to data of pre-vaccination age-specific prevalence of HPV in females in the Netherlands.[27] The target prevalence is described in more details in Section B.2.

The calibration procedure briefly goes as follows. First, the parameters of waning of natural, infection-induced immunity were fixed: $\gamma_{f}=0.1, \gamma_{m}=1$ in the base-case analysis and $\gamma_{f}=$ $0.2, \gamma_{m}=4$ in sensitivity analysis $\mathrm{A}$, which explores the impact of assuming a shorter duration of natural immunity. Then, the transmission probabilities $\left(\beta_{v t}, \beta_{n v t}\right)$ and clearance rates $\left(\mu_{v t}, \mu_{n v t}\right)$ were obtained assuming no competition between the two types, i.e. with the competition parameter $h=1$. Results of this step are shown in Section B.3. The obtained transmission probabilities were fixed subsequently while we re-calibrated the clearance rates to match other pre-fixed values for 
$h(=1.33,1.67, \cdots, 3)$. This step is further described in Section B.4. The parameter values obtained from this entire calibration procedure are shown in Table S6.2

In addition, there were parameters that only affect the post-vaccination transmission dynamics but not the pre-vaccination one, such as the parameters concerning the vaccination scheme and vaccine efficacy. The applied values of these parameters are shown in Section B.5.

In Section B.6, we summarise the differences in applied parameter values between the performed analyses, which are the base-case analysis and the three sensitivity analyses.

Table S6.1: Values of the parameters that were fixed throughout all analyses. * To ease computation, we normalised the birth rate to unity without loss of generality.

\begin{tabular}{ll|r}
\hline \multicolumn{2}{l|}{ Fixed parameters } & Values \\
\hline \hline Demographics & & \\
\hline Birth rate & $m$ & 1 per year* \\
Age of entering sexual mixing population & $a_{\text {enter }}$ & 10 years \\
Age of exiting sexual mixing population & $a_{\text {exit }}$ & 70 years \\
Fraction of female and male & $p_{f}, p_{m}$ & $1 / 2,1 / 2$ \\
Fraction of low- and high-risk groups & $p_{l}, p_{h}$ & $4 / 5,1 / 5$ \\
\hline Contact patterns & & \\
\hline Age of maximum mean contact rate & $a_{\bar{c}_{\max }}$ & 22 years \\
Maximum mean contact rate & $\bar{c}_{\max }$ & 2 persons per year \\
Width of decrease in mean contact rate & $\omega$ & $1 / 2,1 / 2$ \\
Assortative mixing parameters & $r_{l}, r_{h}$ & 0.8 \\
Coefficient of variance & $c_{v}$ & 3 years \\
Maximum mean age difference & $\delta_{\max }$ & 4 years \\
Minimum variance in age of contacts & $\sigma_{\min }^{2}$ & 16 years \\
Maximum variance in age of contacts & $\sigma_{\max }^{2}$ & \\
\hline
\end{tabular}

Table S6.2: Values of parameters that were varied in the base-case analysis, sensitivity analyses A, $\mathrm{B}$, and $\mathrm{C}$. ${ }^{*}$ The values of competition parameter, vaccine-type and non-vaccine-type clearance rates of different parameter sets are shown in a corresponding order, e.g. in the base-case analysis the clearance rates corresponding to $h=1$ were $\mu_{v t}, \mu_{n v t}=0.674,1.178$.

\begin{tabular}{|c|c|c|}
\hline Varied parameters & & Values \\
\hline \multicolumn{3}{|c|}{ Base-case analysis, sensitivity analyses $B, C$} \\
\hline Rate waning natrual immunity in female & $\gamma_{f}$ & 0.1 per year \\
\hline Rate waning natrual immunity in male & $\gamma_{m}$ & 1 per year \\
\hline Transmission probabilities & $\beta_{v t}, \beta_{n v t}$ & $0.45,0.45$ \\
\hline Competition parameter & & $1.00,1.33,1.67,2.00,2.33,2.67,3.00^{*}$ \\
\hline Vaccine-type clearance rate & $\mu_{v t}$ & $0.674,0.655,0.642,0.632,0.624,0.618,0.611^{*}$ \\
\hline Non-vaccine-type clearance rate & $\mu_{n v t}$ & $1.178,1.068,0.997,0.948,0.910,0.882,0.858^{*}$ \\
\hline \multicolumn{3}{|l|}{ Sensitivity analysis $A$} \\
\hline Rate waning natural immunity in female & $\gamma_{f}$ & 0.2 per year \\
\hline Rate waning natural immunity in male & $\gamma_{m}$ & 4 per year \\
\hline Transmission probabilities & $\beta_{v t}, \beta_{n v t}$ & $0.75,0.75$ \\
\hline Competition parameter & $h$ & $1.00,1.33,1.67,2.00,2.33,2.67,3.00^{*}$ \\
\hline Vaccine-type clearance rate & $\mu_{v t}$ & $1.078,1.039,1.011,0.992,0.976,0.964,0.954^{*}$ \\
\hline Non-vaccine-type clearance rate & $\mu_{n v t}$ & $1.923,1.740,1.619,1.532,1.467,1.416,1.376^{*}$ \\
\hline
\end{tabular}

\section{B.2. Target prevalence}

The prevalence of the model vaccine type was calibrated to broadly match the pre-vaccination agespecific prevalence of HPV-16 among females in the Netherlands. 27. This age-specific prevalence is increasing until around age 22 years old and then decreasing, with a peak value of approximately $8 \%$. 

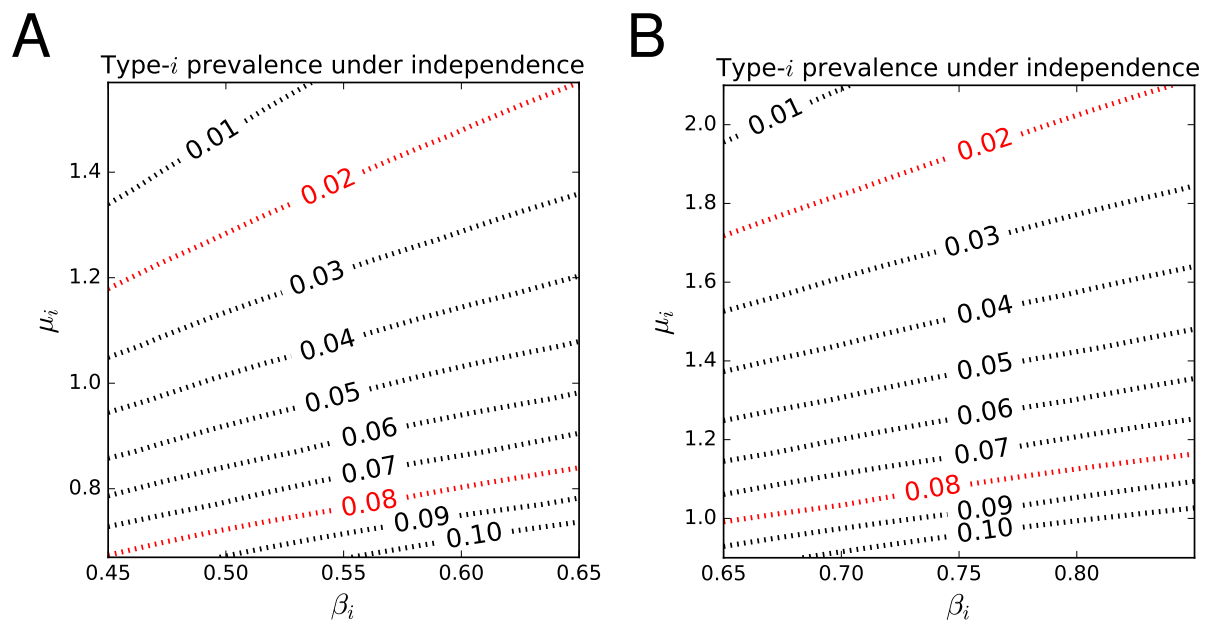

Figure S6.5: Contour plots of the maximum value of the age-specific prevalence belonging to type $i$ assuming no interactions to other types for different combinations of values of $\beta_{i}$ and $\mu_{i}$. The red lines indicate the combinations that were able to reproduce the same maximum value as the target prevalence. The combinations that also reproduced a peak value within the target range are shown in Figure S6.6 A) In the base-case analysis, $\gamma_{f}=0.1$ and $\gamma_{m}=1$. B) In sensitivity analysis A, $\gamma_{f}=0.2$ and $\gamma_{m}=4$.

Accordingly, the maximum value of the model vaccine type prevalence was required to be equal to $8 \%$ in the range $22-24$ years. The target of the model non-vaccine type followed the same curve but was four times smaller, i.e. with a maximum value of $2 \%$.

\section{B.3. Calibration under independence}

We obtained parameter values of the transmission probabilities $\left(\beta_{v t}, \beta_{n v t}\right)$ and clearance rates $\left(\mu_{v t}\right.$, $\mu_{n v t}$ ) that were able to reproduce the target prevalence assuming no competition between types. By assuming independence between the two types, parameters corresponding to either type could be calibrated separately. For both types, we obtained pairs of type-specific transmission probabilities and clearance rates that were able to reproduce the target prevalence. These pairs were characterised by positive correlation, i.e. high values of $\beta_{i}$ needed to be compensated by high values of $\mu_{i}$ Figure S6.5A for the base-case analysis, Figure S6.5 3 for sensitivity analysis A). In addition, the higher the values of a pair were, the smaller the age at the maximum contact rate became [Figure S6.6 for the base-case analysis, Figure S6.7 for sensitivity analysis A). Although more than one parameter set was able to reproduce the age-specific prevalence with the peak falling in the required range ( $22-24$ years), we only chose one of them to continue with in the next calibration steps. For the base-case analysis, the chosen parameter value was $\beta_{v t}=\beta_{n v t}=0.45$. For sensitivity analysis $\mathrm{A}$, it was $\beta_{v t}=\beta_{n v t}=0.75$.

\section{B.4. Calibration under competition}

Using values of transmission probabilities $\left(\beta_{v t}, \beta_{n v t}\right)$ obtained from the calibration step under independence, we then searched for combinations of the clearance rates $\left(\mu_{v t}, \mu_{n v t}\right)$ that were able to reproduce the target prevalence of the vaccine and non-vaccine type simultaneously for assuming 
A

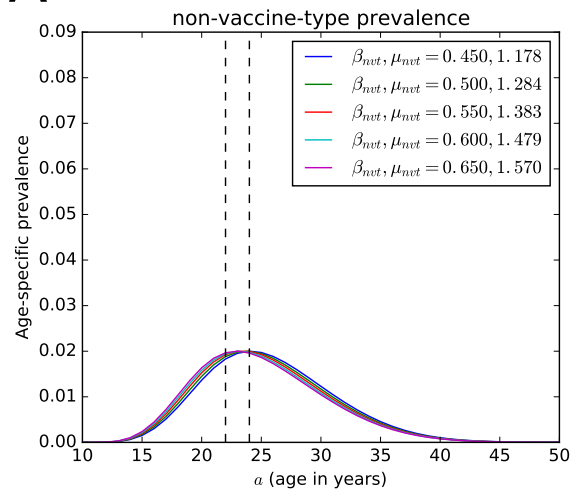

B

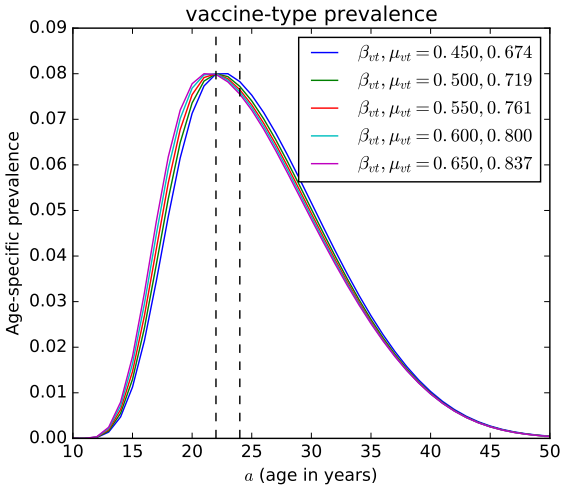

Figure S6.6: (Base-case analysis) Shifting of age-specific prevalence of (A) the vaccine type and (A) the non-vaccine type produced by different combinations of values of $\beta_{v t}, \mu_{v t}$ and $\beta_{n v t}, \mu_{n v t}$, respectively. Increasing values of $\beta_{i}$ and $\mu_{i}$ lead to a shift to earlier age.

A

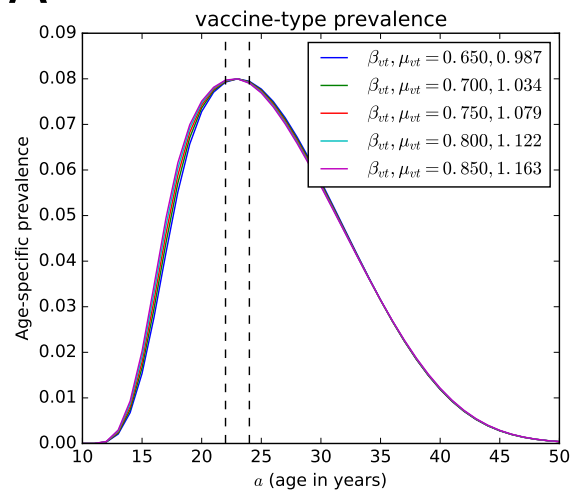

B

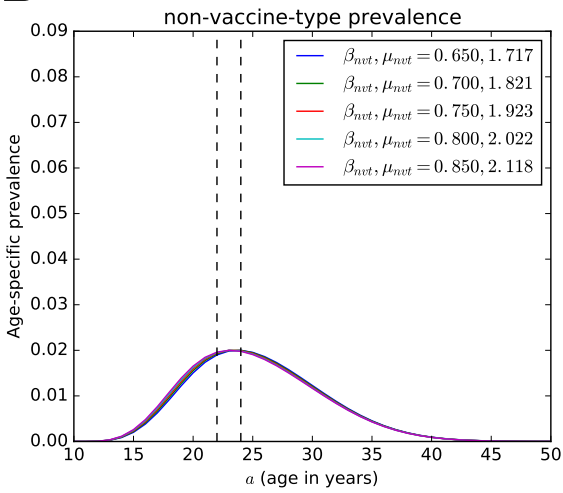

Figure S6.7: (Sensitivity analysis A) Shifting of age-specific prevalence of (a) the vaccine type and (b) the non-vaccine type produced by different combinations of values of $\beta_{v t}, \mu_{v t}$ and $\beta_{n v t}, \mu_{n v t}$, respectively. Increasing values of $\beta_{i}$ and $\mu_{i}$ lead to a shift to earlier age. 
various pre-fixed values of the competition parameter $h>1$. As we previously found, the clearance rates corresponding to $h=1$ were $\mu_{v t}=0.674$ and $\mu_{n v t}=1.178$. The clearance rates we found for other values of the competition parameter $(h=1.33,1.67, \cdots, 3)$ are shown in Table S6.2 Notably, the resulting clearance rates had an inverse relationship with respect to the competition parameter; to retain the same prevalence, a stronger competition needed to be compensated by slower clearance. In Figure S6.8 this inverse relationship can be observed by the shifting of the location where the two red lines cross towards the left lower corner. The same inverse relationship was observed in sensitivity analysis A, where we assumed higher rates of waning of natural immunity [Figure S6.9].

\section{B.5. Vaccination schemes of the different analyses}

In the base-case analysis and sensitivity analysis $\mathrm{A}$, we implemented a girl-only vaccination scheme with high uptake, mimicking the setting in Scotland. In addition, we also implemented two other vaccination schemes for two other sensitivity analyses:

- a girl-only vaccination scheme with low uptake, mimicking the setting in the Netherlands (sensitivity analysis $\mathrm{B}$ );

- a sex-neutral vaccination scheme with higher uptake, mimicking the setting in Australia (sensitivity analysis $\mathrm{C}$ ).

Each of the following subsections describes one of the three vaccination schemes.

\section{B.5.1. High-uptake vaccination scheme}

The high-uptake vaccination scheme of the base-case analyse and sensitivity analysis A mimicking the Scottish setting consists of a regular girls-only vaccination program at age 12 years with $95 \%$ uptake and a catch-up program at the introduction of vaccination for girls up to 18 years old [Figure S6.10 A). (42. The vaccination coverages in different catch-up cohorts were:

- age $13-14$ years: $80 \%$;

- age 15-16 years: $60 \%$;

- age $17-18$ years: $40 \%$.

\section{B.5.2. Low-uptake vaccination scheme}

The low-uptake vaccination scheme of sensitivity analysis B mimicking the Dutch setting consists of a regular girls-only vaccination program at age 12 years with $60 \%$ uptake and a catch-up program at the introduction of vaccination for girls up to 18 years old [Figure S6.10,4). [43] The vaccination coverages in different catch-up cohorts were:

- age 13-14 years: $50 \%$;

- age 15-16 years: $40 \%$;

- age $17-18$ years: $30 \%$.

\section{B.5.3. Excessively-high-uptake vaccination scheme}

The excessively-high-uptake vaccination scheme of sensitivity analysis $C$ mimicking the Australian setting consists of a girls-only program at the introduction of vaccination. After six years of vaccination, the program was extended to boys with stable uptake of $80 \%$ (Figure S6.103).[44]. The regular program for girls is at age 12 years with $80 \%$ uptake, complemented by a catch-up program at the introduction of vaccination for girls up to 18 years old. The vaccination coverages in different catch-up cohorts were:

- age 13-14 years: $70 \%$;

- age 15-16 years: $60 \%$;

- age 17-18 years: $50 \%$. 

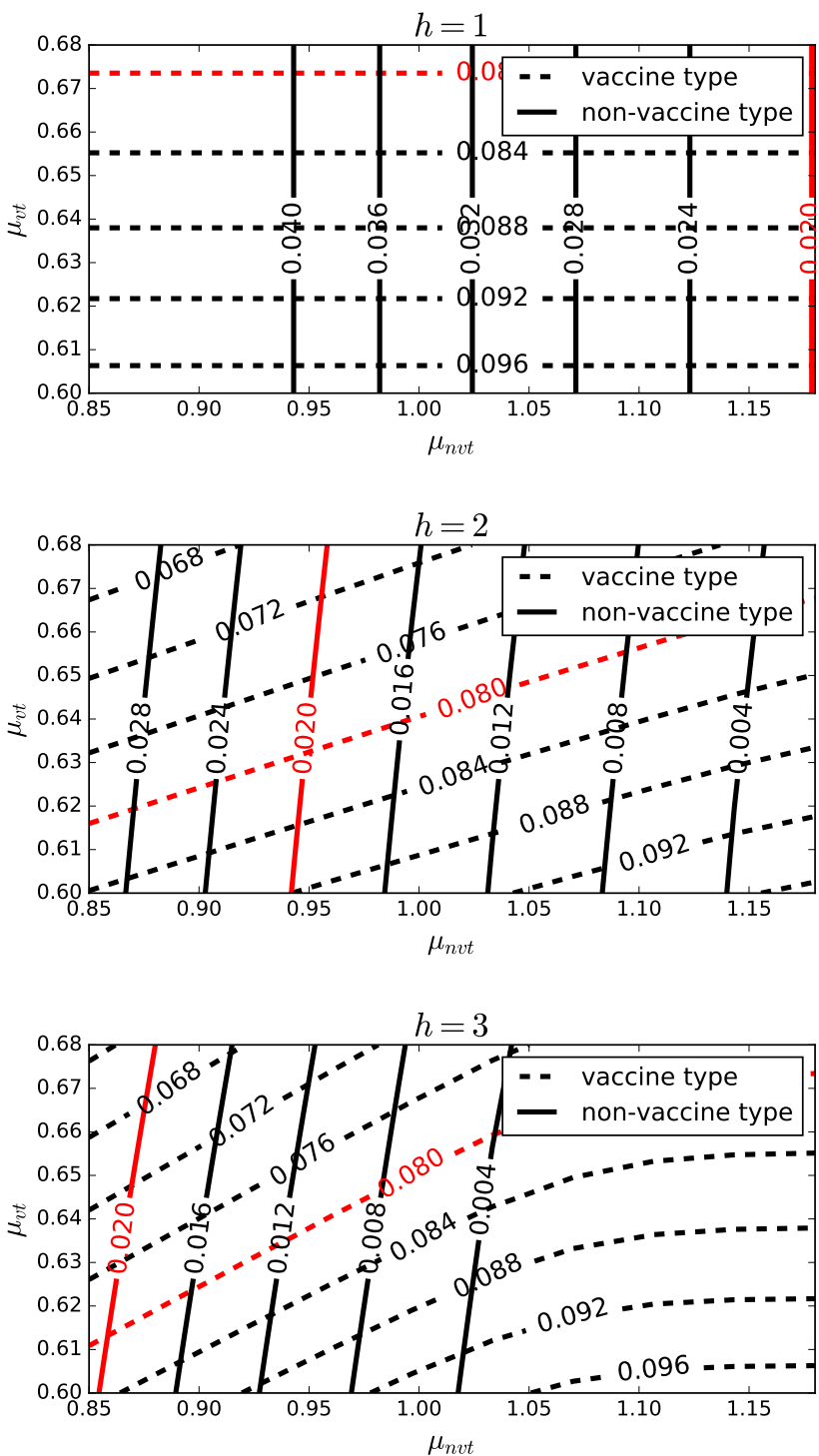

Figure S6.8: (Base-case analysis) Contour plots of the maximum values of the age-specific prevalence belonging the vaccine (dashed lines) and non-vaccine (solid lines) type for different combinations of $\mu_{v t}$ and $\mu_{n v t}$. Each subfigure corresponds to a different value of the competition parameter $h(=1,2,3)$. The red contour lines correspond to the maximum values of the target prevalence. 

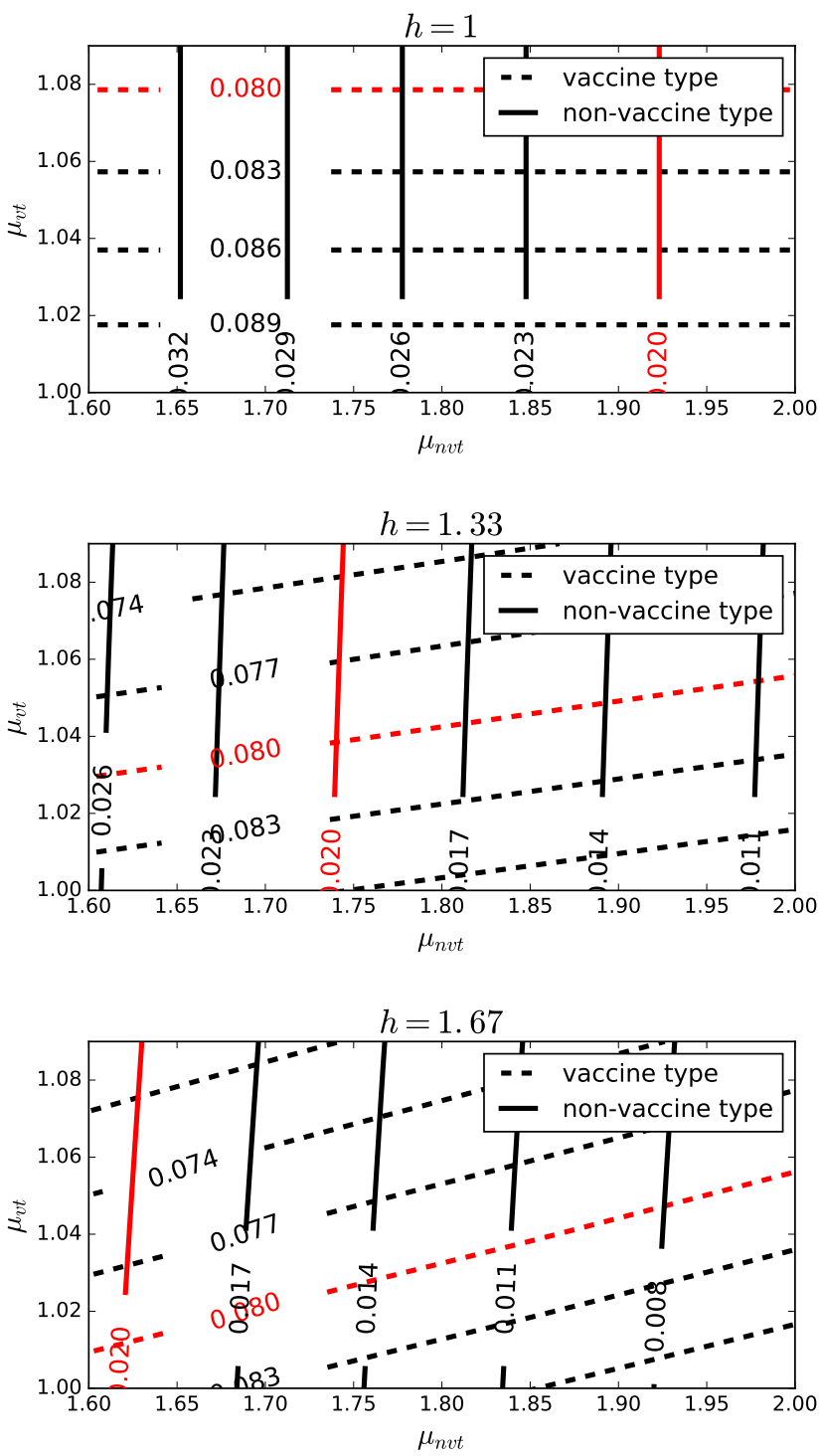

Figure S6.9: (Sensitivity analysis A) Contour plots of the maximum values of the age-specific prevalence belonging the vaccine (dashed lines) and non-vaccine (solid lines) type for different combinations of $\mu_{v t}$ and $\mu_{n v t}$. Each subfigure corresponds to a different value of the competition parameter $h(=1,2,3)$. The red contour lines correspond to the maximum values of the target prevalence. 

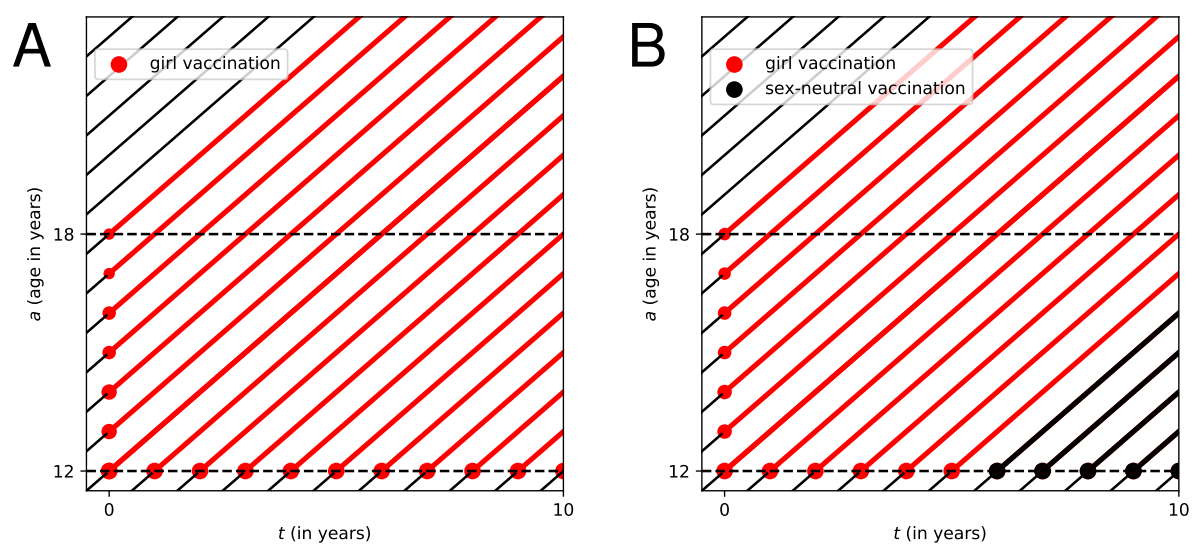

Figure S6.10: Lexis diagrams depicting the vaccinated cohorts and vaccination moments in (a) the base-case analysis, sensitivity analyses A, B and (b) sensitivity analysis C. Girl-only and sex-neutral vaccinated cohorts are indicated in red and black, respectively. Time points of vaccination are indicated by circles.

\section{B.6. Overview of the analyses and results}

The section summarises the key differences in the parameter values between the performed analyses. In the base-case analysis, the waning rates of naturally acquired, i.e. infection-induced, immunity in females and males were set to $\gamma_{f}=0.1$ and $\gamma_{m}=1$, respectively. The vaccination scheme was implemented with high uptake as described in Section B.5.1. In sensitivity analysis A, the rates of waning of natural immunity in females and males were higher: $\gamma_{f}=0.2$ and $\gamma_{m}=4$. The vaccination scheme was identical to the one in the base-case analysis. In sensitivity analysis $B$, the rates of waning natural immunity were changed back to the values of the base-case analysis, while the vaccination scheme was implemented with low uptake one as described in Section B.5.2. Lastly, in sensitivity analysis $\mathrm{C}$, the vaccination scheme was implemented with excessively high uptake as described in Section B.5.3. See Figure S6.11 for a schematic diagram summarizing the parameter values used in the different analyses.

The values of the parameter obtained from the calibration process concerning the transmission probabilities $\left(\beta_{v t}, \beta_{n v t}\right)$, the clearance rates $\left(\mu_{v t}, \mu_{n v t}\right)$, and the competition parameter $(h)$ for the different analyses can be found in Table S6.2

\section{Simulation results}

This chapter presents the simulation results across different analyses (defined in Section B.6) and is organised as follows. Section C.1 shows the age-specific prevalence over time since the introduction of vaccination. Section C.2 presents the results regarding short-term type replacement for different age groups. In Section C.3, we present the correspondence between, on one hand, final type replacement and, on the other hand, short-term type replacement or vaccine effectiveness for the non-vaccine type. 


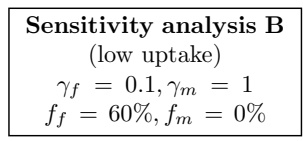

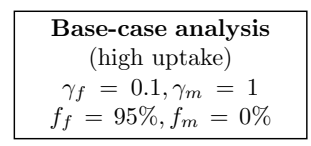

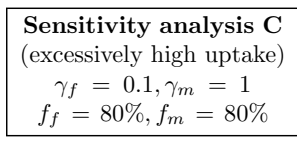

Sensitivity analysis A
$\gamma_{f}=0.2, \gamma_{m}=4$
$f_{f}=95 \%, f_{m}=0 \%$

Figure S6.11: A schematic diagram summarizing the parameter values that characterise the basecase analysis and sensitivity analyses $\mathrm{A}, \mathrm{B}$, and $\mathrm{C}$. Here, $f_{f}$ and $f_{m}$ denote the coverage of the regular vaccination scheme, whereas $\gamma_{f}$ and $\gamma_{m}$ denote the rate of natural immunity in female and male, respectively.

\section{C.1. Age-specific prevalence over time}

In this section, we present the age-specific prevalence of the vaccine and non-vaccine types over time. The prevalence of the vaccine and non-vaccine type are presented in separate figures:

- vaccine-type prevalence at $t=0,5,10,15,25,50$ years since vaccination Figure S6.12 Figure S6.15 Figure S6.18 Figure S6.21;

- non-vaccine-type prevalence at $t=0,5,10,15,25,50$ years since vaccination Figure S6.13 Figure S6.16 Figure S6.19 Figure S6.22;

In addition, there are separate figures showing non-vaccine-type prevalence at only $t=0$ and $t=$ 50 since vaccination to better visualise the difference of prevalence in the pre- and post-vaccination equilibria Figure S6.14 Figure S6.17)|Figure S6.20 Figure S6.23. To ease the comparison to Figure 6.2 and 6.4 in the main text, the subfigures of all figures in this section are arranged in the same grid format; the $\mathrm{x}$-axis corresponds to the level of competition $(h)$ and the $y$-axis the level of crossprotection $\left(\theta_{n v t}\right)$. Furthermore, in the figures with the non-vaccine-type prevalence at $t=0,50$ years, we indicated in red frames the scenarios where 1) the non-vaccine-type prevalence had decreased among young ages and increased among old ages at $t=50$ as compared to $t=0$, and 2) final type replacement occurred at the population level.

Throughout all analyses, the post-vaccination equilibrium was reached within 50 years. In the base-case analysis, and sensitivity analyses $A$ and $C$, the uptake was high enough to eliminate the vaccine type in the post-vaccination equilibrium, but it was not the case in sensitivity analysis $B$ [Figure S6.12 Figure S6.15 Figure S6.18 Figure S6.21.

\section{C.2. Type replacement across age groups}

In the main text, we defined short-term type replacement to be an increase in non-vaccine-type prevalence among $a$-year-old women at $t$ years since the introduction of vaccination as compared to the corresponding prevalence in the pre-vaccination equilibrium. The corresponding prevalence ratio is denoted by $P R(a, t)$. In this section, we present this prevalence ratio evaluated at different ages over time.

\section{C.2.1. Base-case analysis}

We first discuss the results obtained in the base-case analysis. In the absence of cross-protection and competition, the non-vaccine-type prevalence remained unchanged for all age groups (flat blue lines in Figure S6.24. When there was competition (but still in absence of cross-protection), the prevalence ratio corresponding to short-term type replacement $(P R(a, t))$ increased for approximately the 
first 18 years of vaccination. After a slight overshoot, the prevalence ratio stabilised at the new postvaccination equilibria. Moreover, the qualitative behaviour was uniform across all age groups in the sense that, within each scenario (each colour), if the prevalence ratio increased in one age group, it also increased in other age groups.

When the level of cross-protection was set to $\theta_{n v t}=40 \%$, the prevalence ratio first decreased in young age groups (top row of Figure S6.25. After 8 to 12 years, the prevalence ratio either continued to decrease or rebounded, depending on the strength of competition. In the discussion of the main text, we called the initial period of decrease a honeymoon period before type replacement. Such a honeymoon period was not observed in older age groups (bottom row of Figure S6.25. Hence, in the presence of cross-protection, the qualitative behaviour of the prevalence ratio differs across age groups; within each scenario (each colour), the prevalence ratio may increase in some age groups but decrease in other age groups.

\section{C.2.2. Sensitivity analyses}

In the sensitivity analyses, where natural immunity was shorter or coverage was different, we encountered the same result of differences in qualitative behaviour among different age groups whenever there was cross-protection [Figure S6.25] Figure S6.25] and Figure S6.28.

\section{C.3. Evaluating final type replacement with short-term measures}

In this section, we present the results regarding final type replacement in the sensitivity analyses (the results of the base-case analysis are presented in the main text). We then check how well final type replacement corresponds with short-term type replacement and vaccine effectiveness for the nonvaccine type evaluated after 10 years of vaccination.

\section{C.3.1. Final type replacement}

In the main text, we defined final type replacement as an increase in non-vaccine-type prevalence in all women throughout the population in the post-vaccination equilibrium as compared to the corresponding prevalence in the pre-vaccination equilibrium ( $t=0$ years), with final type replacement expressed using prevalence ratio $P R^{f i n a l}$. As shown in Section C.1, the post-vaccination equilibrium was reached within 50 years throughout all simulations. Hence, we evaluated final type replacement at $t=50$ years.

Firstly, when comparing the base-case analysis and sensitivity analysis $A$, where the duration of natural immunity was shorter, we found a similar scope of final type replacement.

Regarding the other sensitivity analyses on the vaccination coverage, we note that increasing uptake increased the scope of final type replacement; the red area shrinks when comparing the top panels of Figure S6.30 and Figure S6.31 to Figure 6.2 of the main text. Moreover, increasing vaccination uptake above the level of vaccine-type elimination expands the amount of cross-protection in the population, while the extent of type replacement is already saturated. Hence, further increasing uptake then decreases the scope and effect size of final type replacement.

\section{C.3.2. Evaluation using short-term type replacement}

We now contrast the findings of final type replacement to the corresponding short-term type replacement evaluated after 10 years among 20-year-old and 25-year-old women. In sensitivity analysis A (shorter natural immunity), due to the faster occurrence of type replacement, evaluation of final type replacement using short-term type replacement after 10 years of vaccination performed better than in the base-case analysis (compare Figure S6.29 to Figure 6.4 in the main text).

In sensitivity analysis B (lower uptake), the scope of final type replacement was larger than in the base-case analysis, while the evaluation of short-term type replacement was similar. Hence, there were more scenarios with final type replacement that were missed by the evaluation using short-term type replacement than in the base-case analysis (compare Figure S6.30|to Figure 6.4 in the main text). 
Conversely, in sensitivity analysis $C$ (higher uptake), the scope of final type replacement was smaller than in the base-case analysis, while the evaluation of short-term type replacement was similar. Hence, short-term type replacement evaluated after 10 years of vaccination was better (compare Figure S6.31 to Figure 6.4 in the main text).

Peculiarly, in sensitivity analysis C, there were a number of scenarios (e.g. $h=3$ and $\theta_{n v t}=40 \%$ ) in which short-term type replacement occurred after 10 years among 25-year-old-women, while final type replacement did not occur in the long run. The reason for this discrepancy is the later inclusion of boys vaccination. In these scenarios, final type replacement would have occurred if vaccination remained for girls only (check Figure 6.2 in the main text). The additional benefits of also vaccinating boys only became apparent after approximately 15 years of vaccination [Figure S6.28.

C.3.3. Evaluation using vaccine effectiveness for the non-vaccine type

In the main text, we defined vaccine effectiveness (VE) as the reduction in the risk of non-vaccinetype infection in vaccinated relative to unvaccinated individuals. It was also defined among women and could be evaluated among different ages and time points. Here, it was evaluated after 10 years among 20-year-old or 25-year-old women.

Throughout all sensitivity analyses, VE for the non-vaccine type among 20-year-old women corresponded poorly with the occurrence of final type replacement but better among 25-year-old women Figure S6.29 Figure S6.30 and Figure S6.31. However, the higher sensitivity among older ages did last long in all scenarios. Figure S6.32 shows the evaluation of VE among different ages and at different time points. For each age group, the sensitivity decreased as the time since vaccination increased. Eventually, VE only expresses the level of cross-protection. As a result, VE always converges to a non-negative value so that it becomes immaterial for indicating type replacement. For example, among 25-year-old women, the sensitivity for detecting final type replacement was almost negligible after 20 years of vaccination. 
Vaccine-type age-specific prevalence
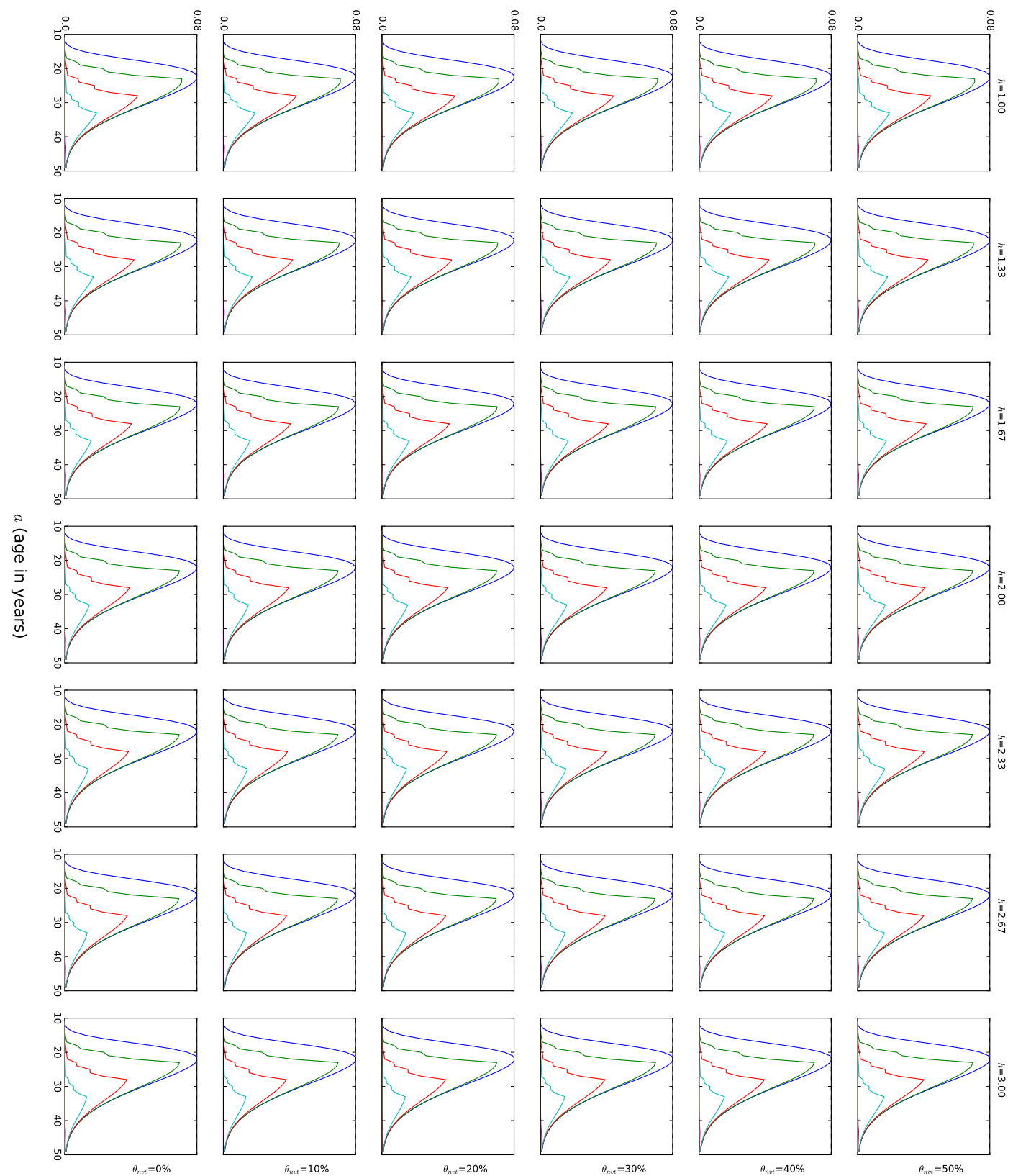

Figure S6.12: (Base-case analysis) Vaccine-type age-specific prevalence at $t=0,5,10,15,25,50$ years (blue, green, red, cyan, purple, yellow) (blue, green, red, cyan, purple, yellow) since vaccination. 
Non-vccine-type age-specific prevalence
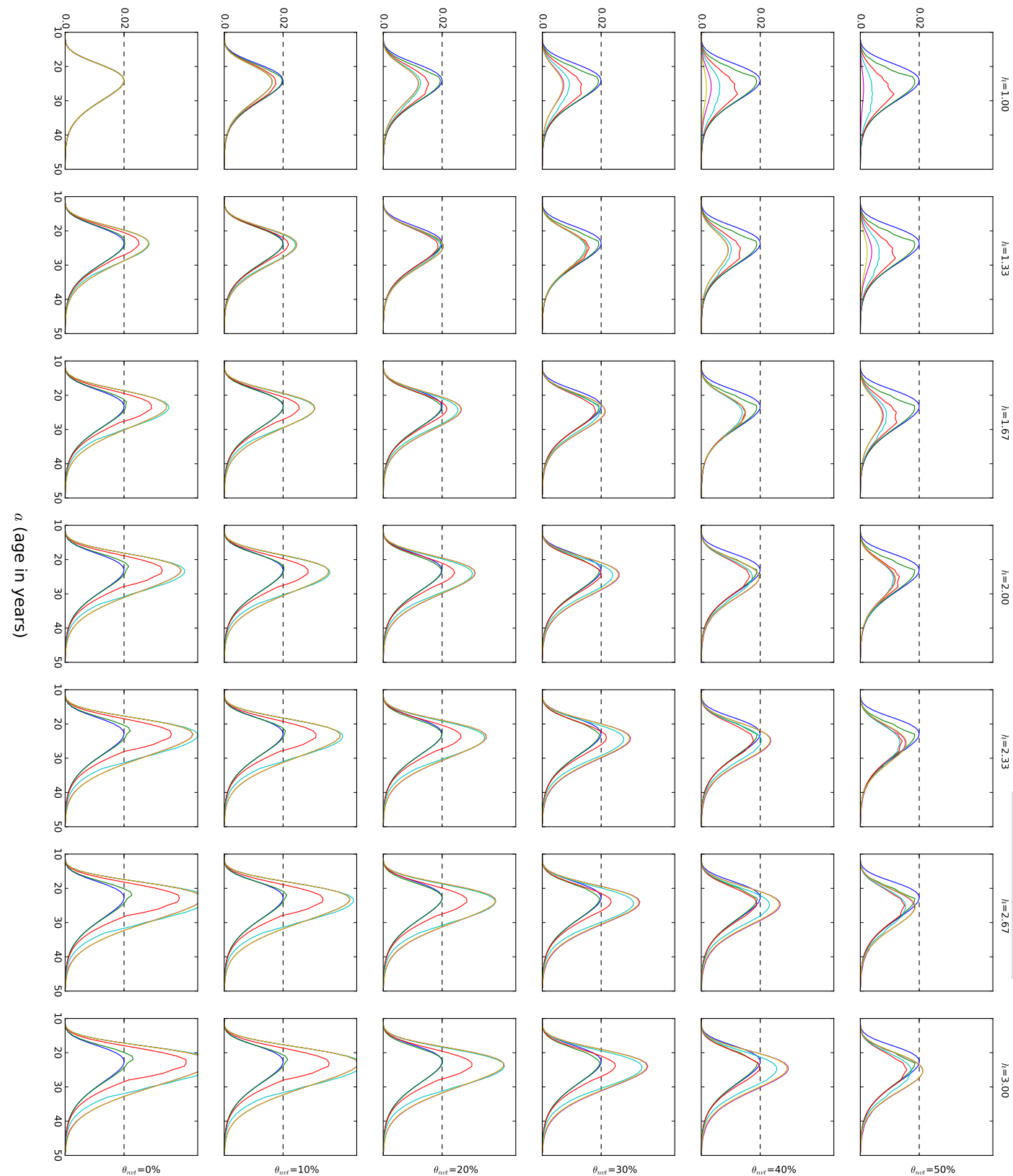

Figure S6.13: (Base-case analysis) Non-vaccine-type age-specific prevalence at $t=$ $0,5,10,15,25,50$ years (blue, green, red, cyan, purple, yellow) since vaccination. 
Non-vccine-type age-specific prevalence
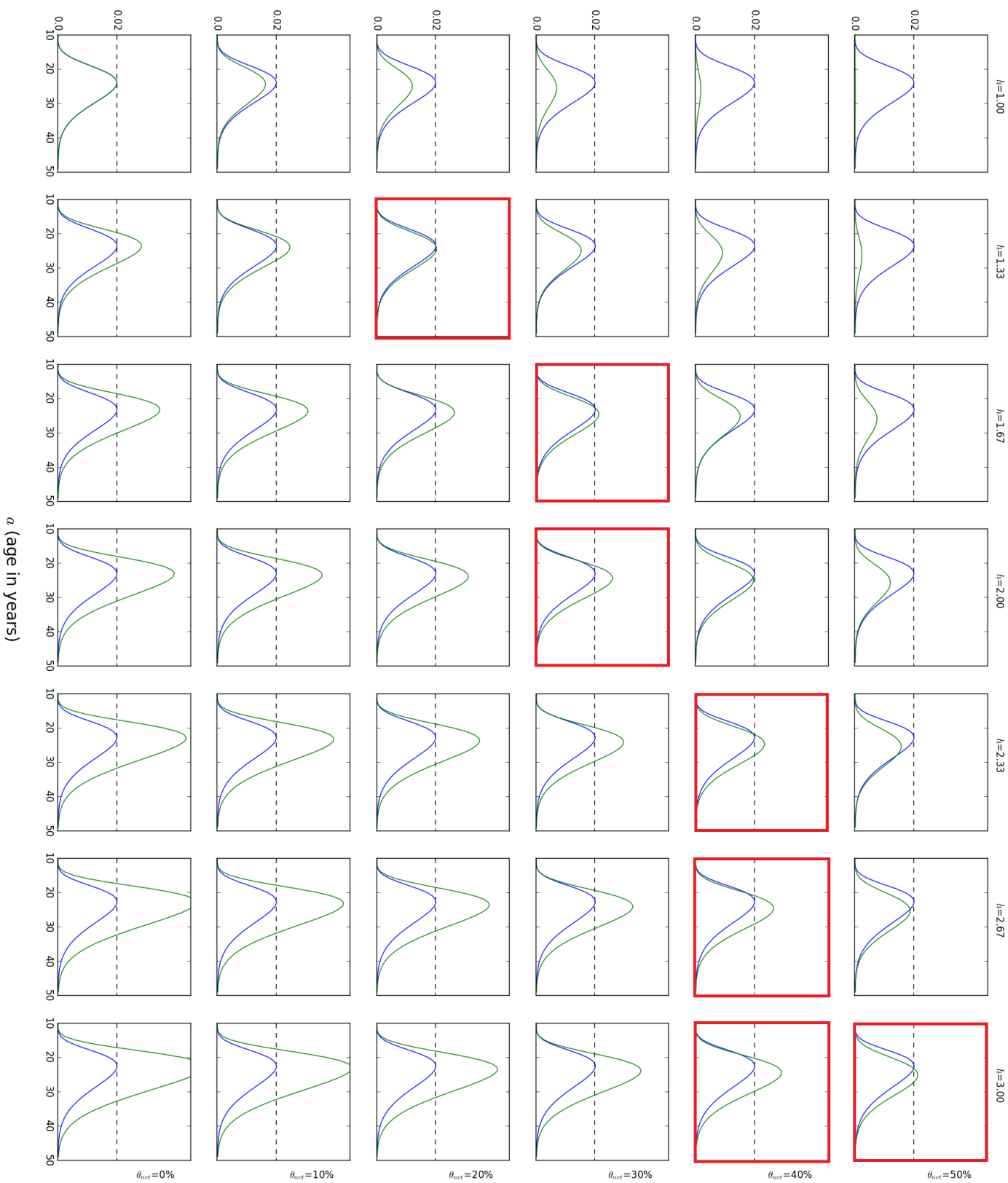

Figure S6.14: (Base-case analysis) Non-vaccine-type age-specific prevalence at the prevaccination ( $t=0$ years, blue) and post-vaccination $(t=50$ years, green) equilibria. Indicated in red are the scenarios where the non-vaccine-types prevalence had decreased among young ages but increased among old ages while final type replacement occurred. 
Vaccine-type age-specific prevalence
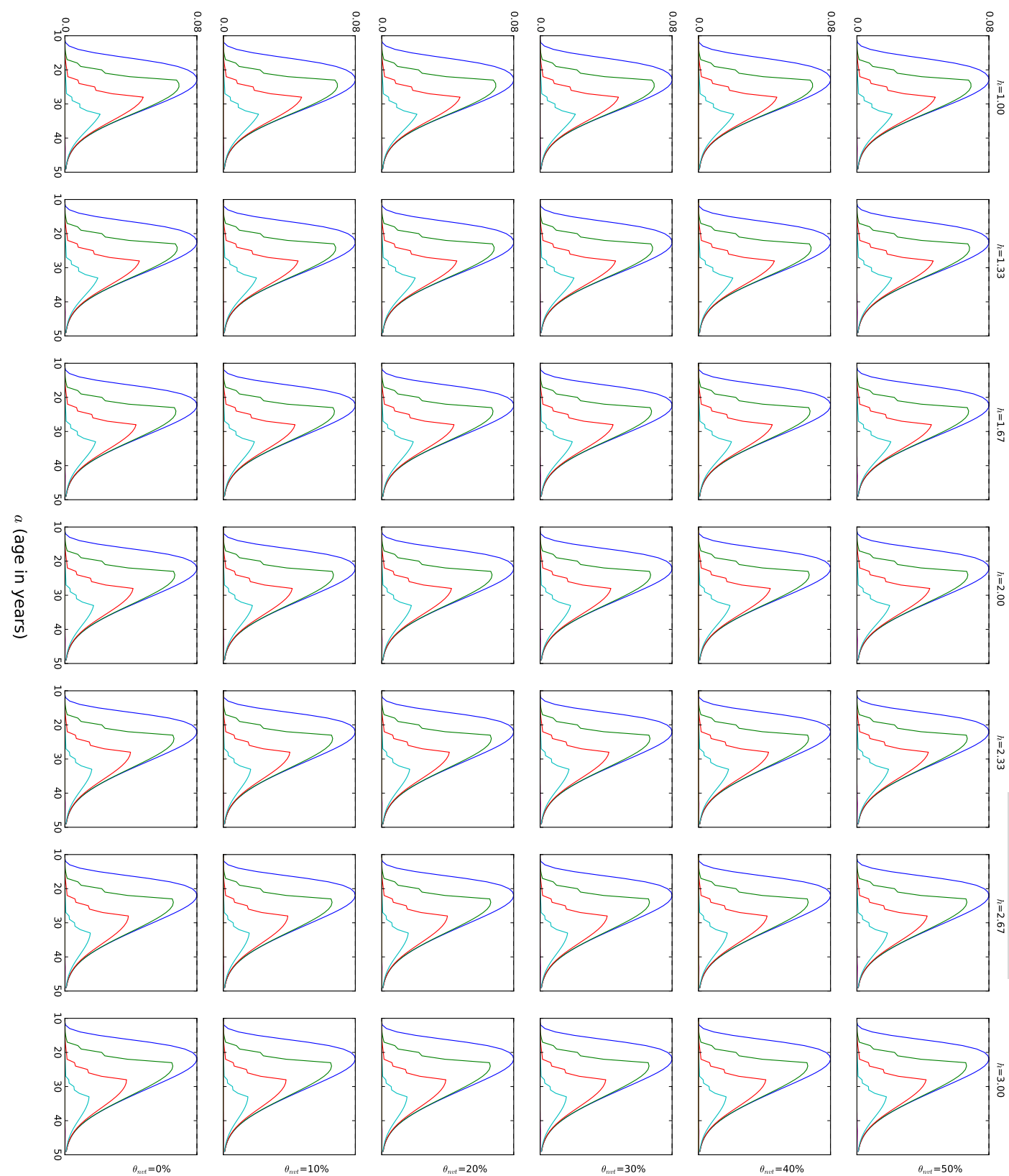

Figure S6.15: (Sensitivity analysis A) Vaccine-type age-specific prevalence at $t=$ $0,5,10,15,25,50$ years (blue, green, red, cyan, purple, yellow) since vaccination. 
Non-vccine-type age-specific prevalence
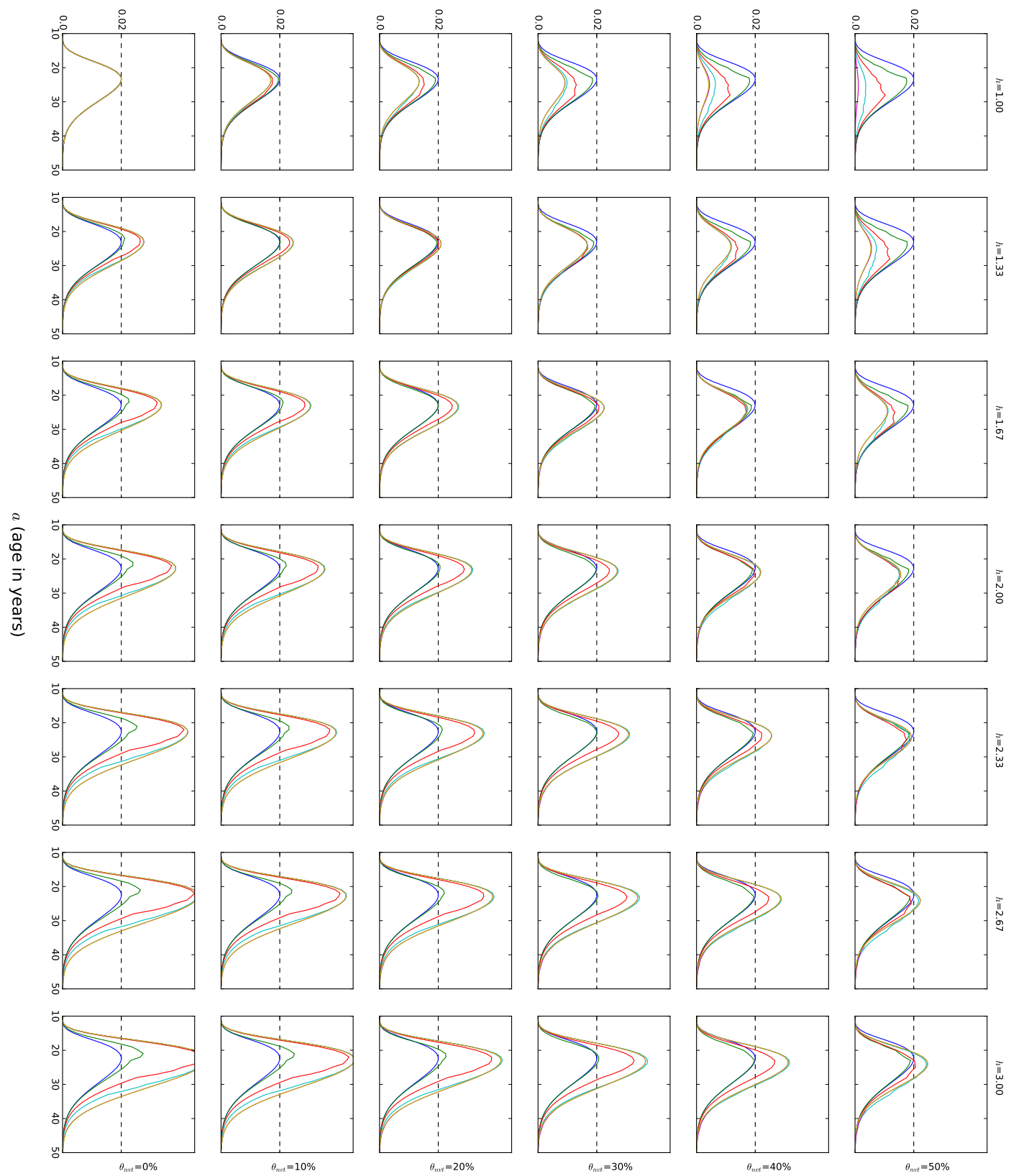

Figure S6.16: (Sensitivity analysis A) Non-vaccine-type age-specific prevalence at $t=$ $0,5,10,15,25,50$ years (blue, green, red, cyan, purple, yellow) since vaccination. 
Non-vccine-type age-specific prevalence
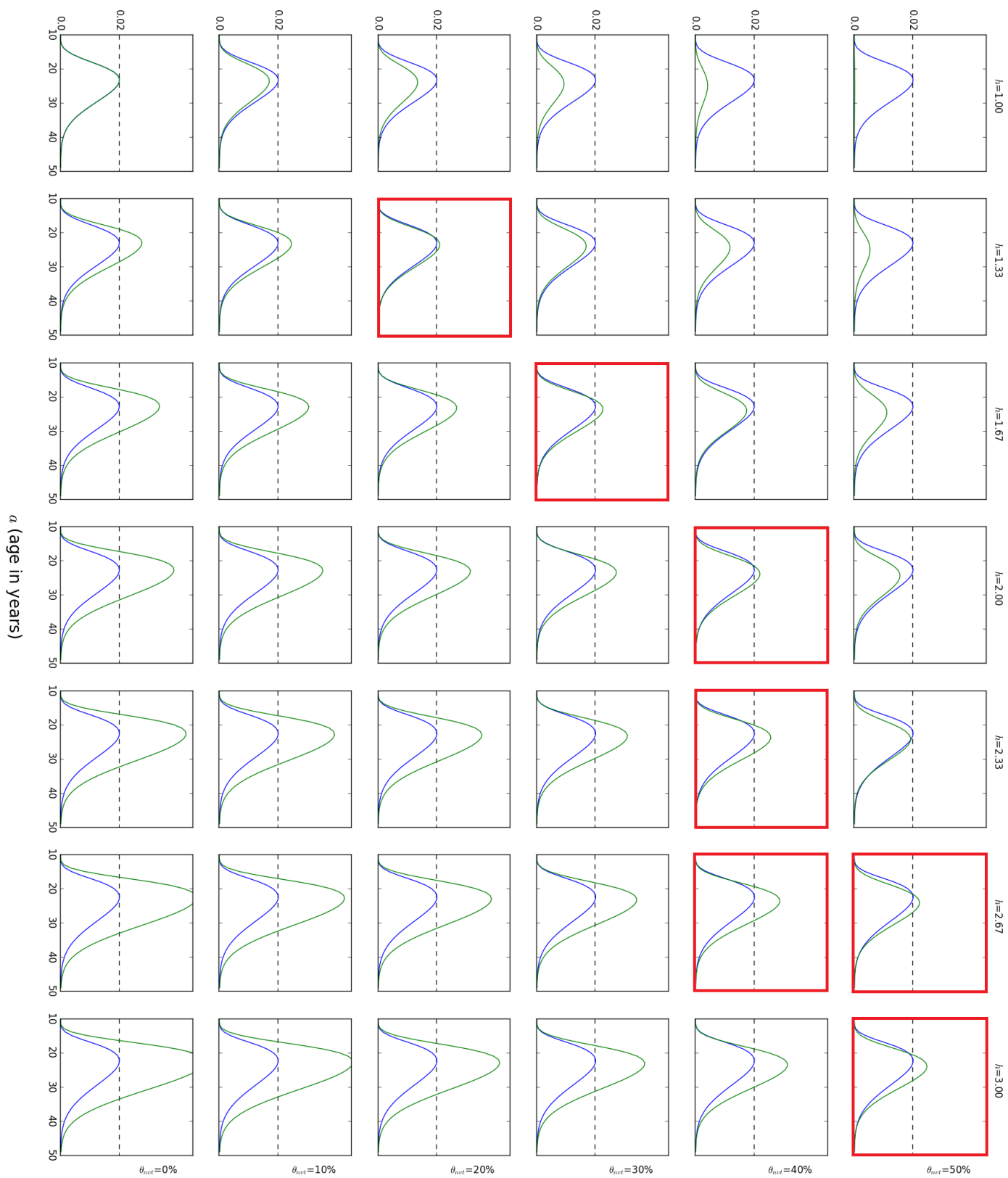

Figure S6.17: (Sensitivity analysis A) Non-vaccine-type age-specific prevalence at the prevaccination ( $t=0$ years, blue) and post-vaccination ( $t=50$ years, green) equilibria. Indicated in red are the scenarios where the non-vaccine-types prevalence had decreased among young ages but increased among old ages while final type replacement occurred. 
Vaccine-type age-specific prevalence
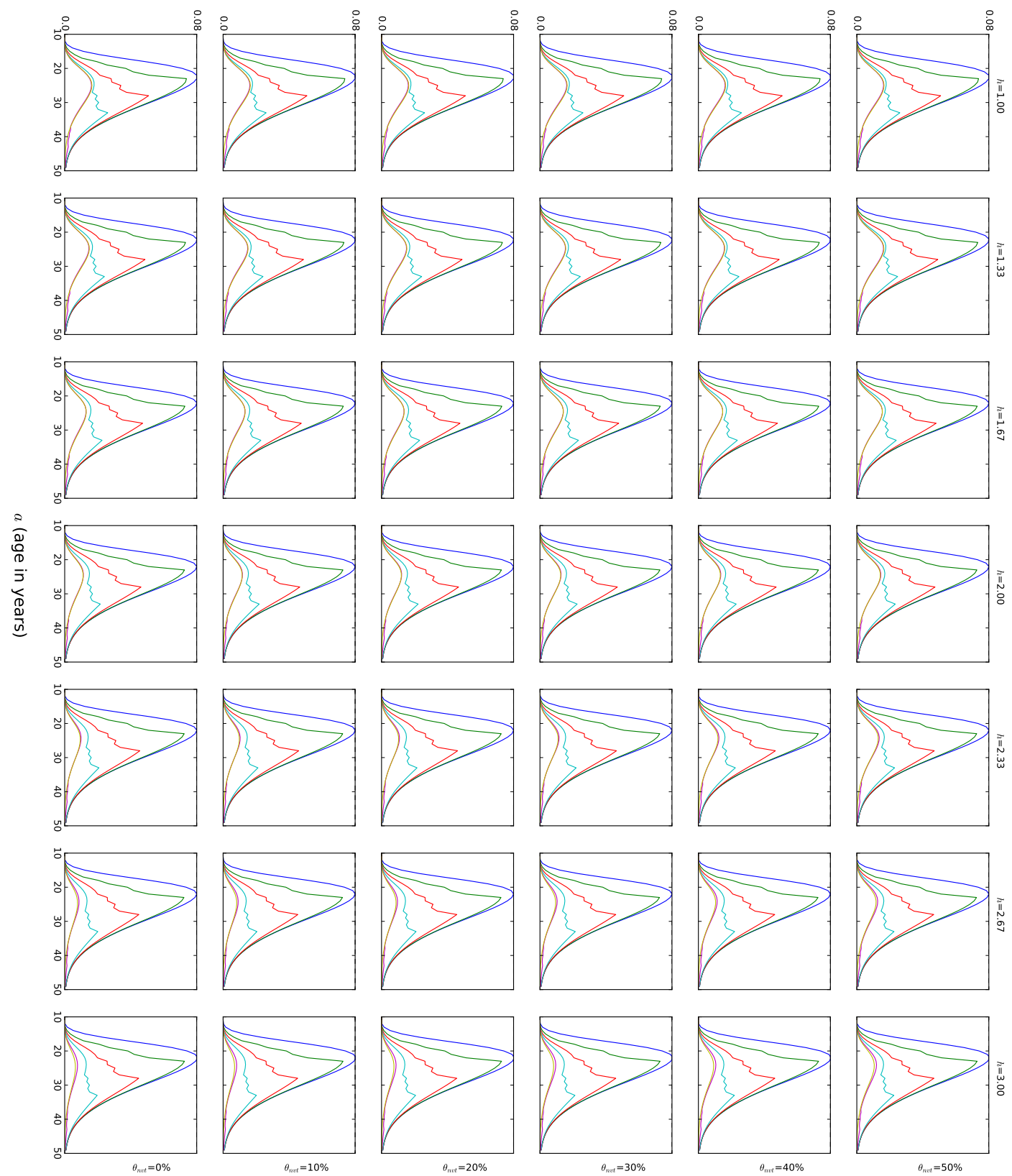

Figure S6.18: (Sensitivity analysis B) Vaccine-type age-specific prevalence at $t=$ $0,5,10,15,25,50$ years (blue, green, red, cyan, purple, yellow) since vaccination. 
Non-vccine-type age-specific prevalence
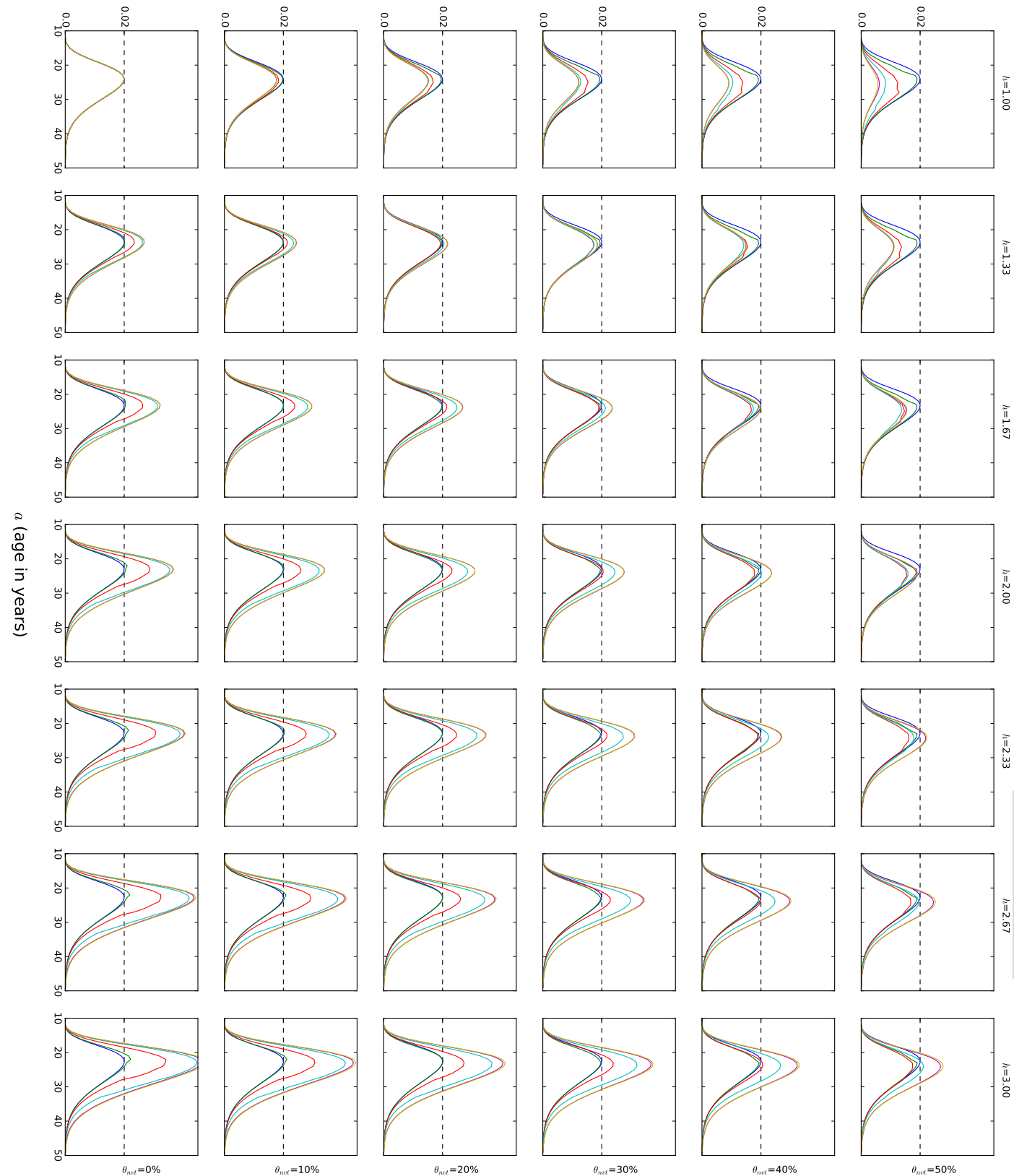

Figure S6.19: (Sensitivity analysis B) Non-vaccine-type age-specific prevalence at $t=$ $0,5,10,15,25,50$ years (blue, green, red, cyan, purple, yellow) since vaccination. 
Non-vccine-type age-specific prevalence
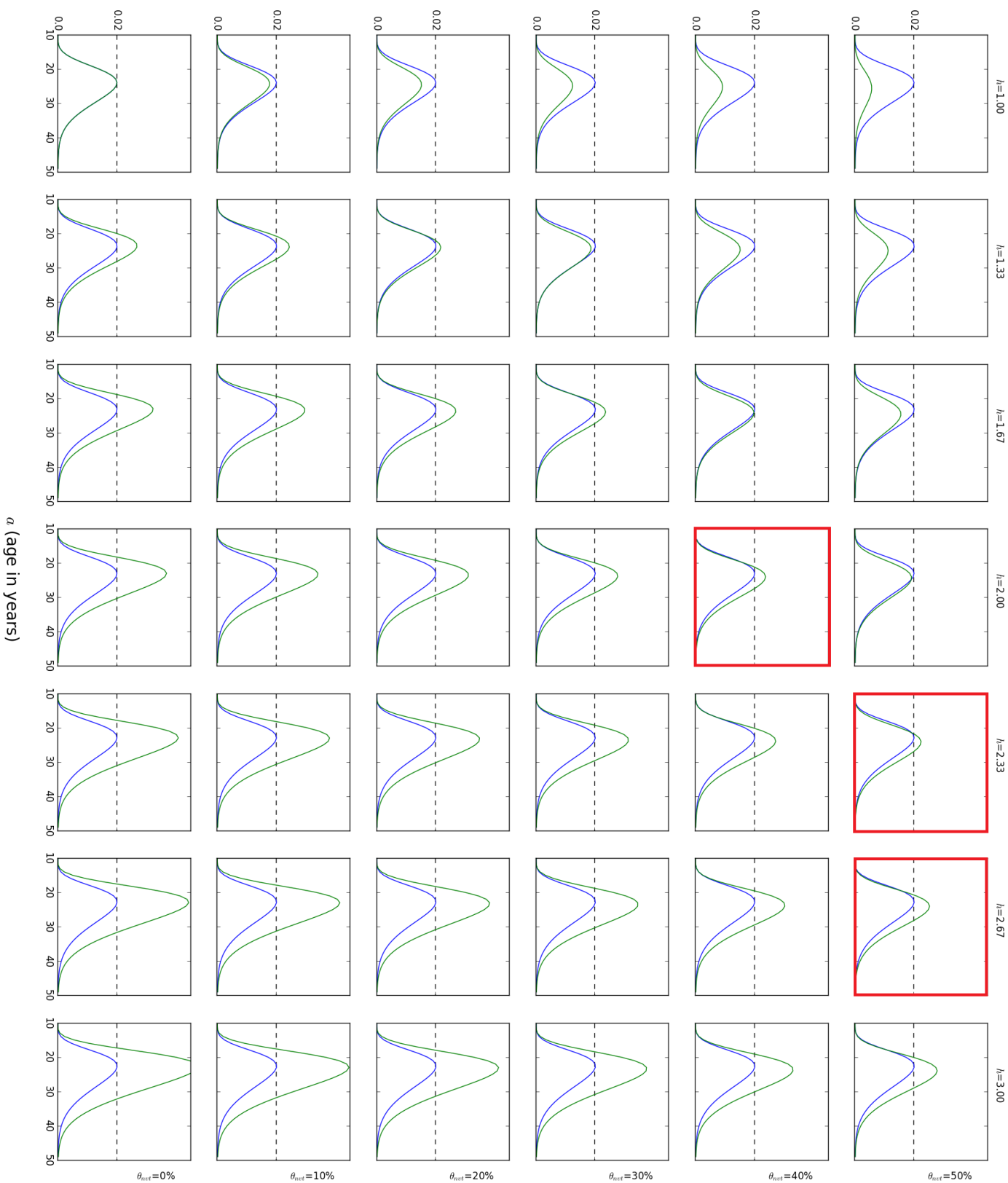

Figure S6.20: (Sensitivity analysis B) Non-vaccine-type age-specific prevalence at the prevaccination ( $t=0$ years, blue) and post-vaccination ( $t=50$ years, green) equilibria. Indicated in red are the scenarios where the non-vaccine-types prevalence had decreased among young ages but increased among old ages while final type replacement occurred. 
Vaccine-type age-specific prevalence
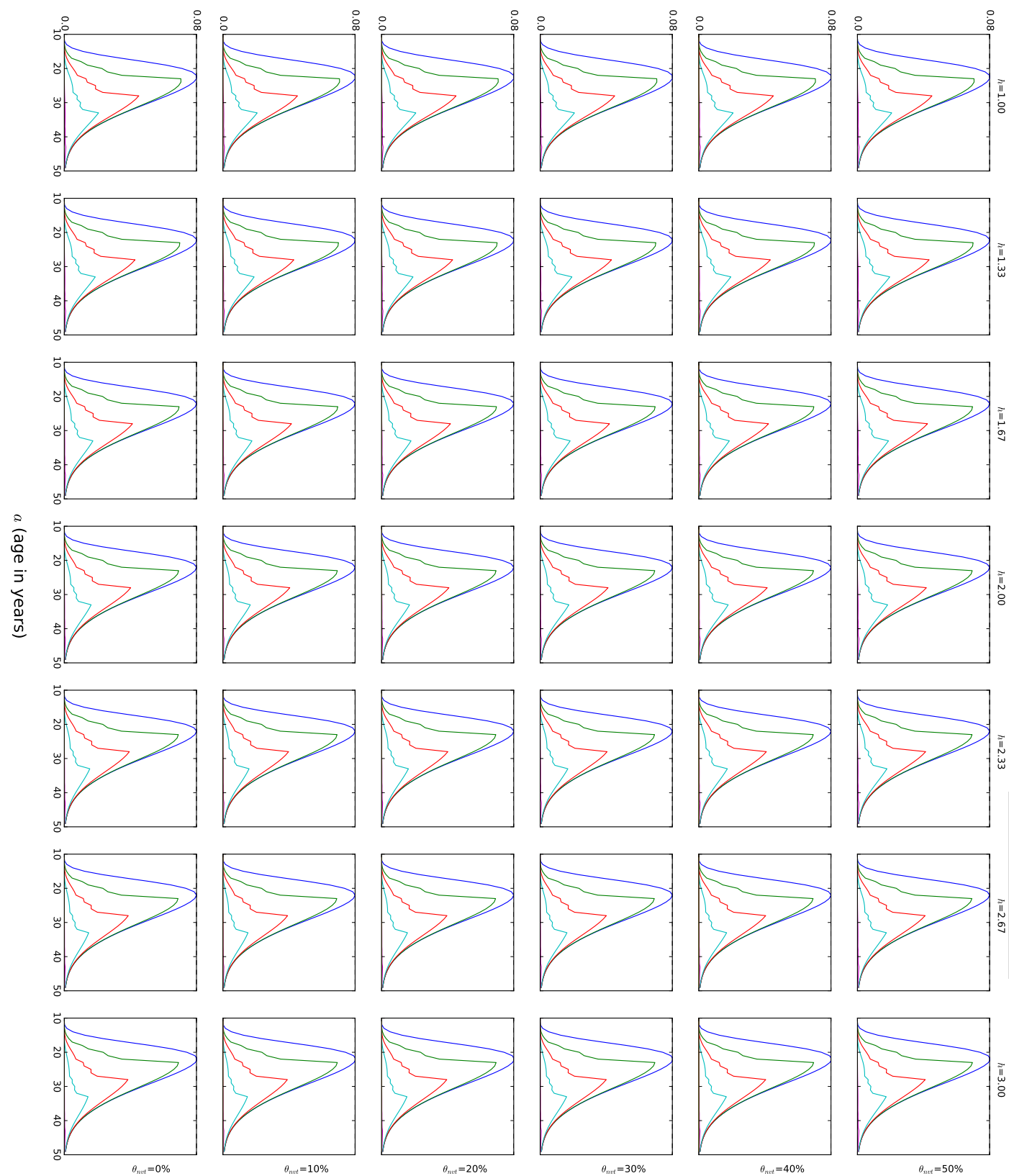

Figure S6.21: (Sensitivity analysis C) Vaccine-type age-specific prevalence at $t=$ $0,5,10,15,25,50$ years (blue, green, red, cyan, purple, yellow) since vaccination. 
Non-vccine-type age-specific prevalence
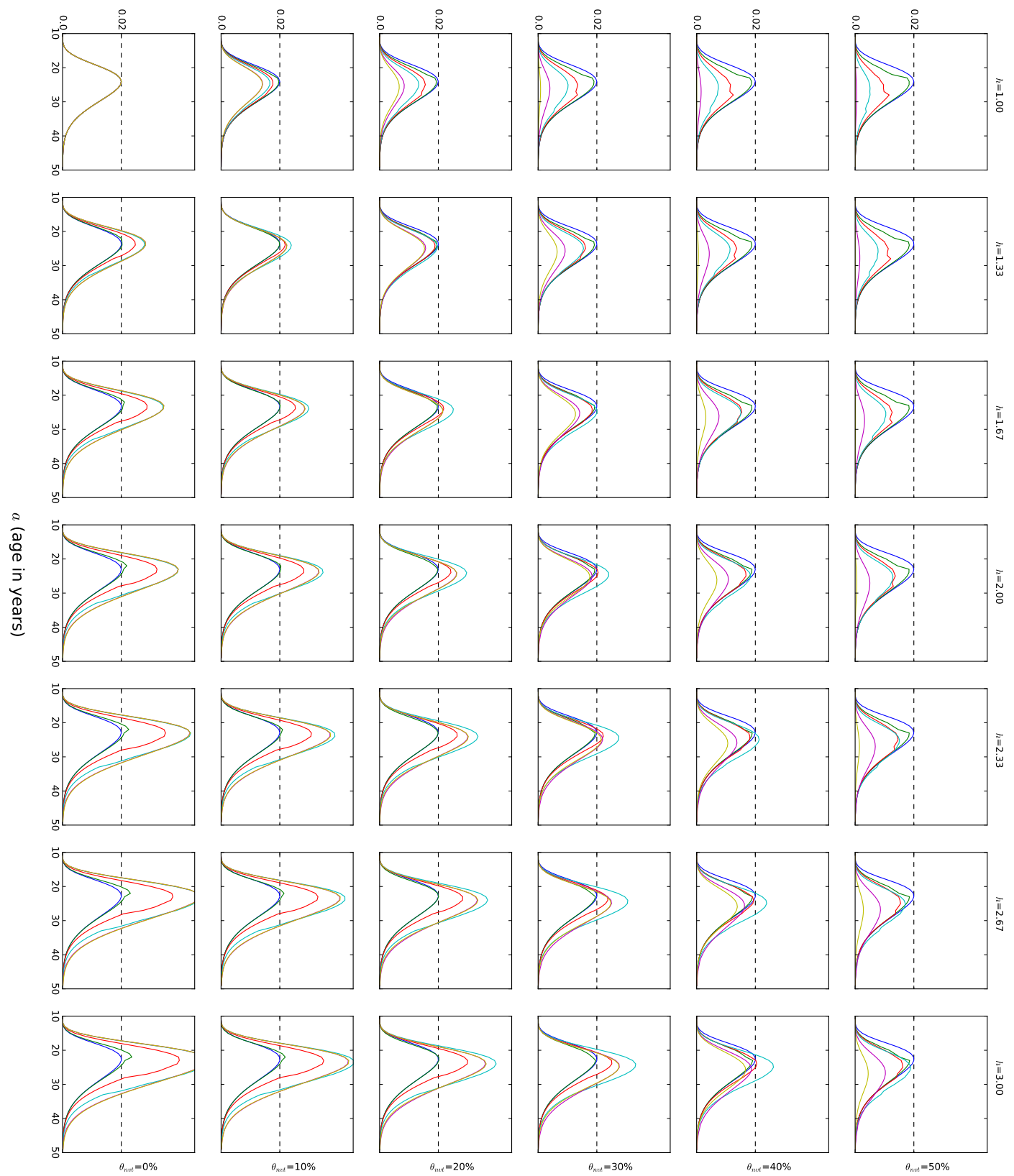

Figure S6.22: (Sensitivity analysis C) Non-vaccine-type age-specific prevalence at $t=$ $0,5,10,15,25,50$ years (blue, green, red, cyan, purple, yellow) since vaccination. 
Non-vccine-type age-specific prevalence
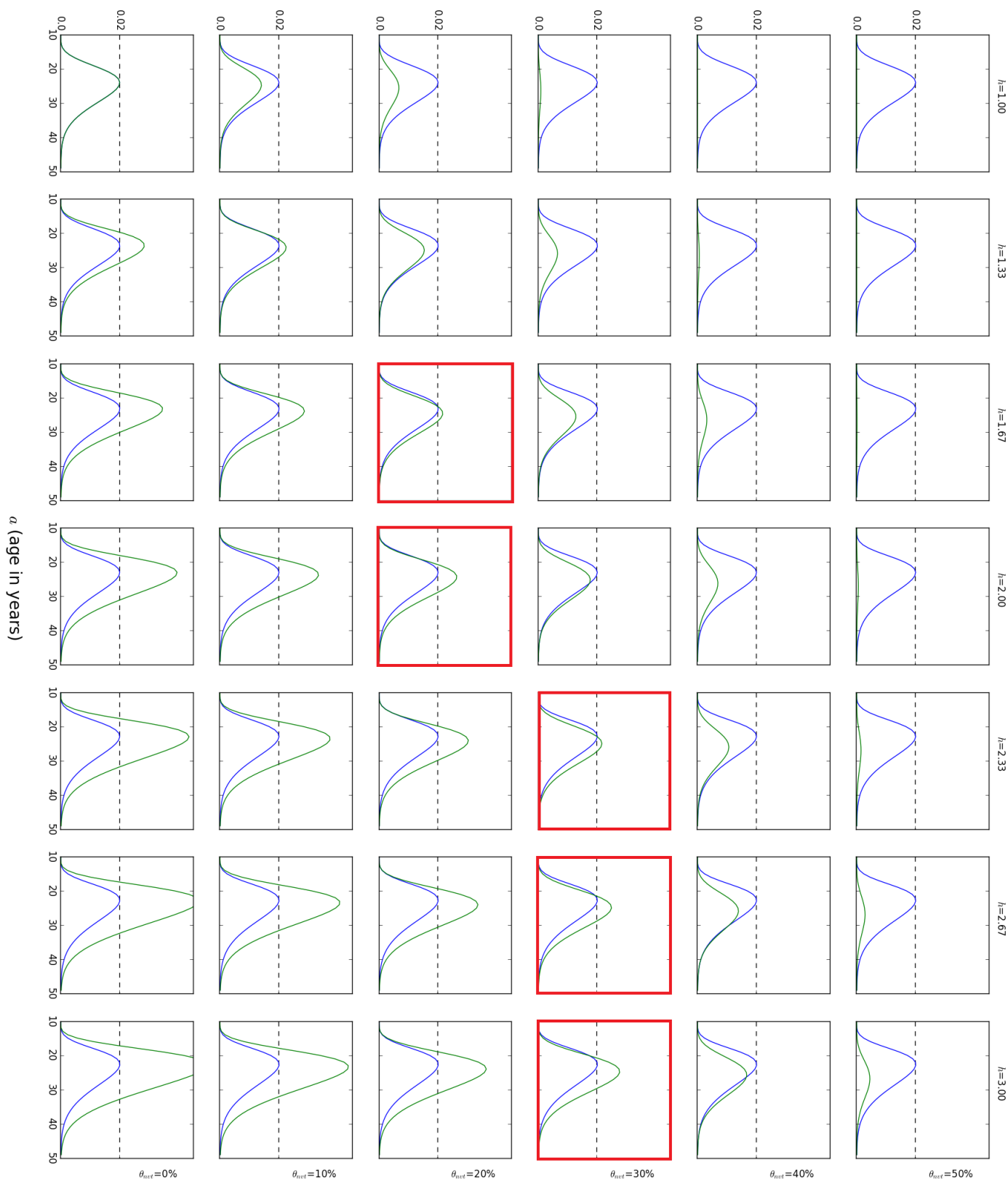

Figure S6.23: (Sensitivity analysis $\mathrm{C}$ ) Non-vaccine-type age-specific prevalence at the prevaccination ( $t=0$ years, blue) and post-vaccination ( $t=50$ years, green) equilibria. Indicated in red are the scenarios where the non-vaccine-types prevalence had decreased among young ages but increased among old ages while final type replacement occurred. 

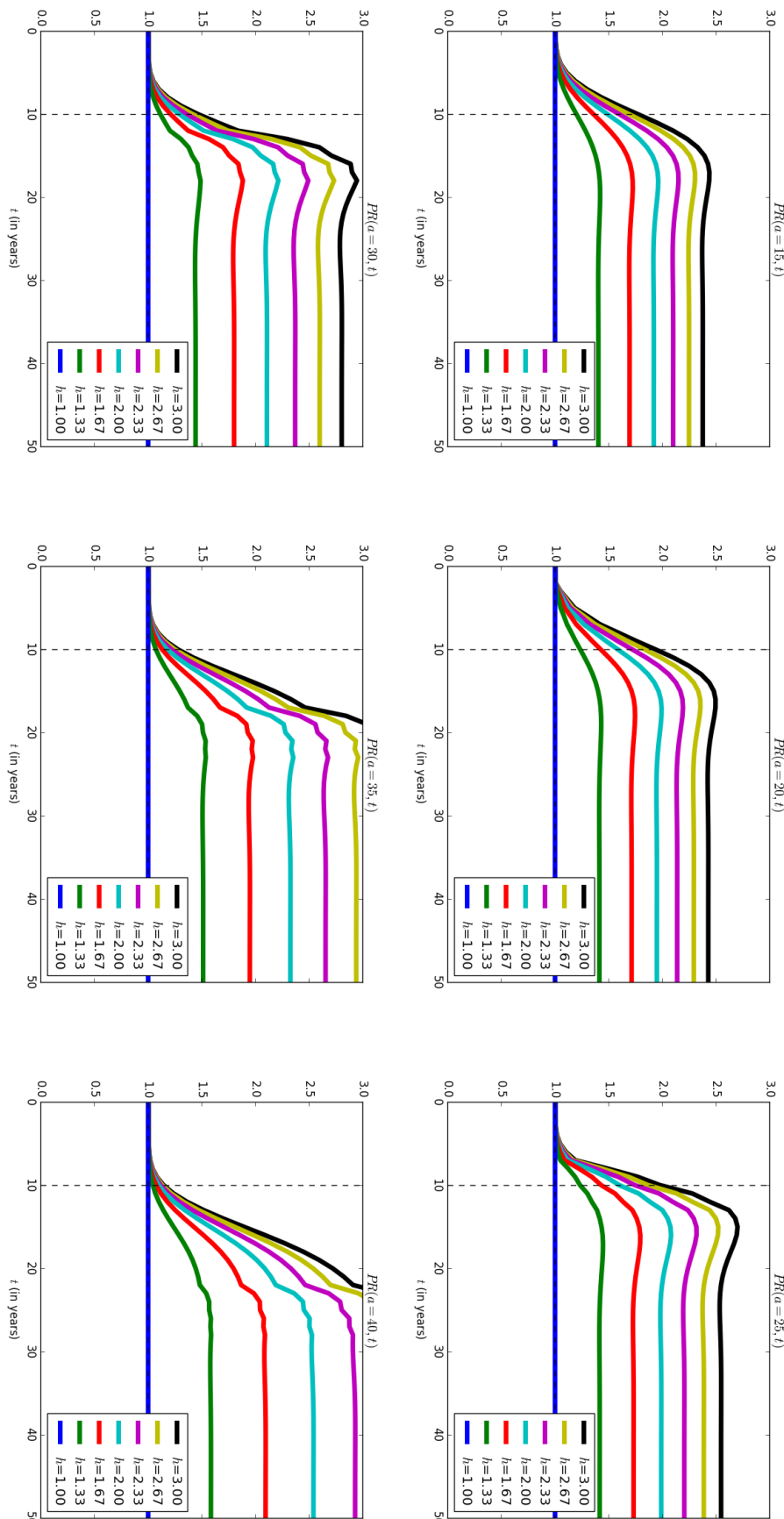

Figure S6.24: (Base-case analysis) Prevalence ratio $P R(a, t)$ over time since the introduction of vaccination evaluating short-term type replacement. Each panel shows $P R(a, t)$ for a different age $a \in\{15,20,25,30,35,40\}$. Different levels of competition $h$ are indicated by different colours. The level of cross-protection was $\theta_{n v t}=0 \%$. 

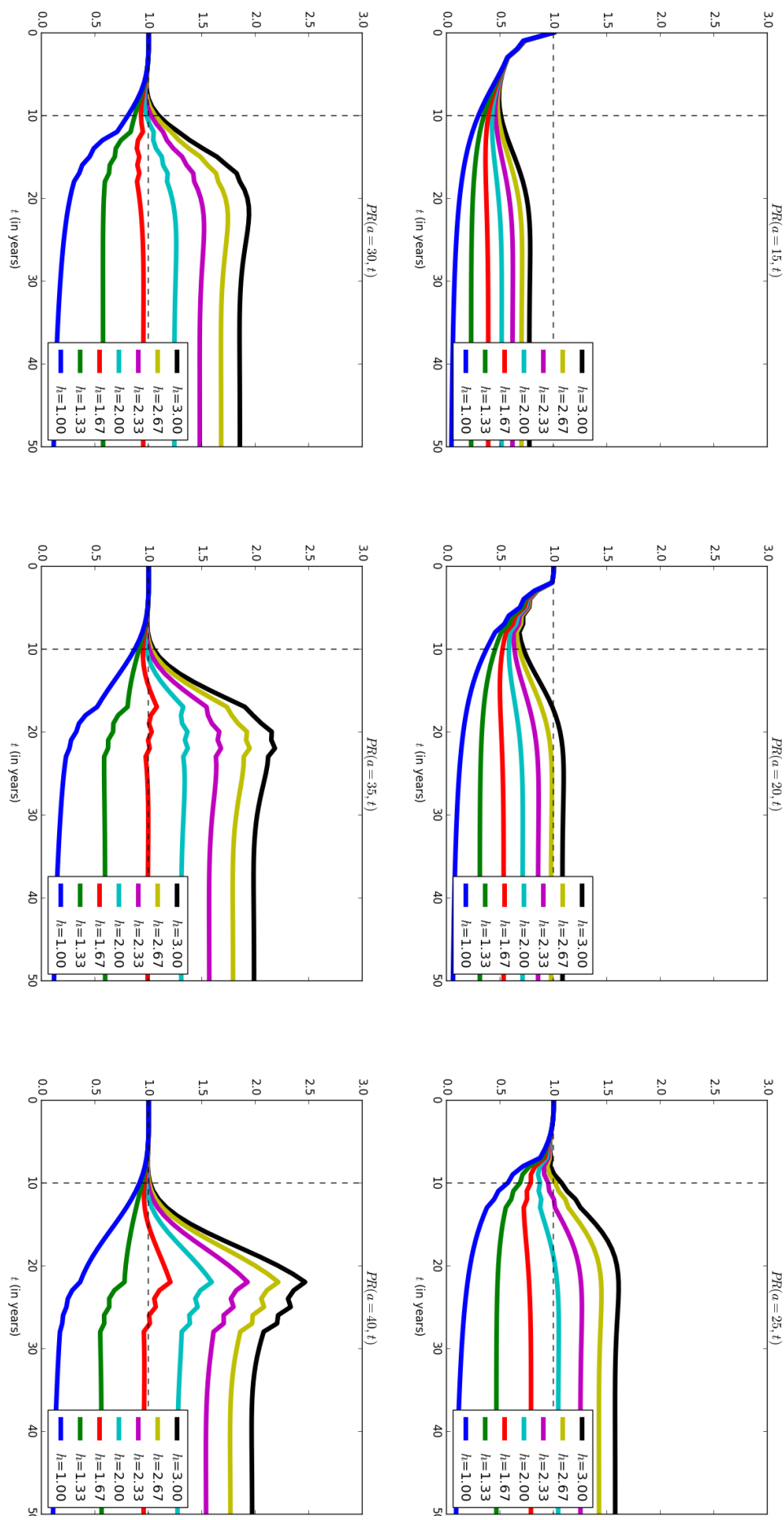

Figure S6.25: (Base-case analysis) Prevalence ratio $P R(a, t)$ over time since the introduction of vaccination evaluating short-term type replacement. Each panel shows $\operatorname{PR}(a, t)$ for a different age $a \in\{15,20,25,30,35,40\}$. Different levels of competition $h$ are indicated by different colours. The level of cross-protection was $\theta_{n v t}=40 \%$. 

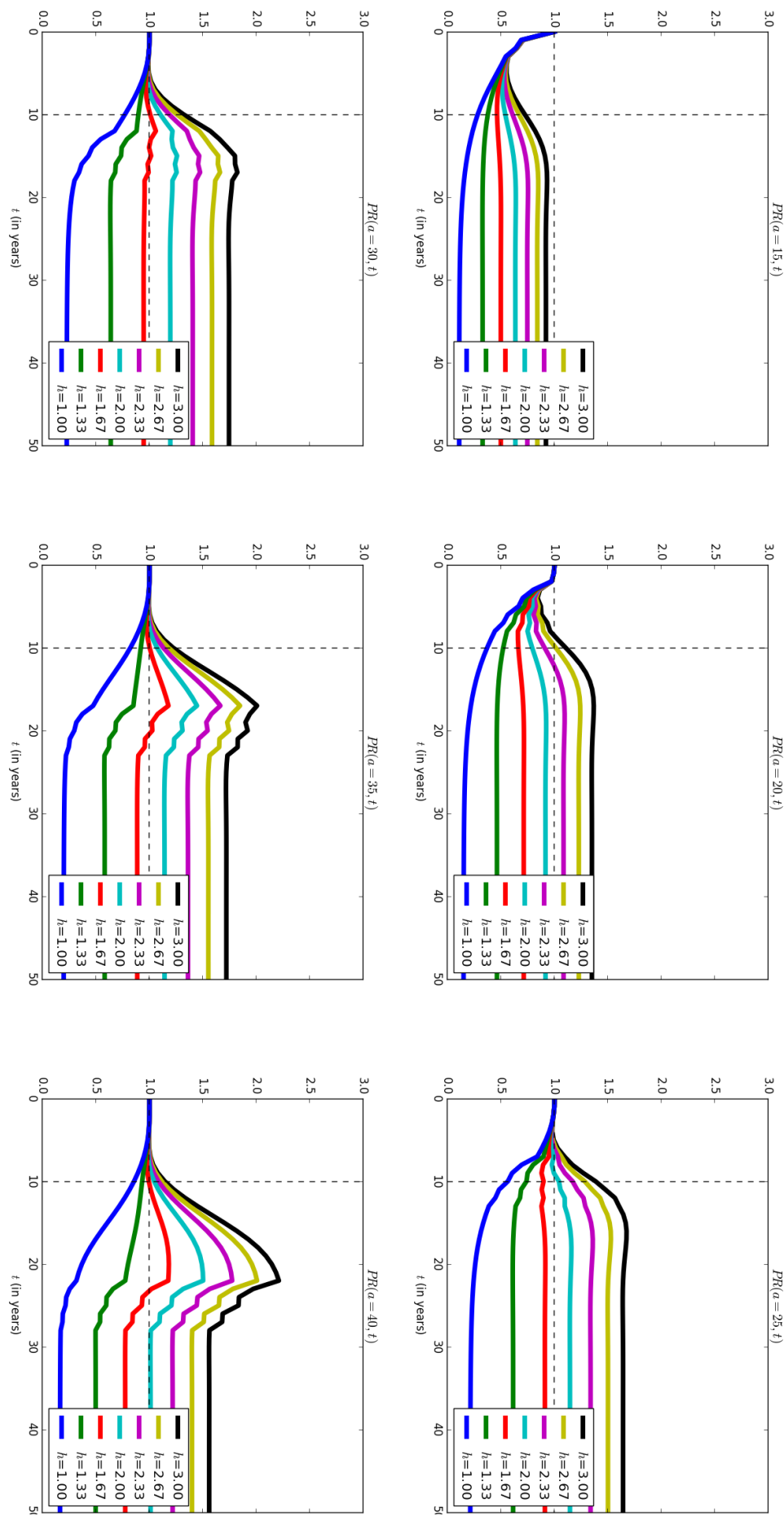

Figure S6.26: (Sensitivity analysis A) Prevalence ratio $P R(a, t)$ over time since the introduction of vaccination evaluating short-term type replacement. Each panel shows $P R(a, t)$ for a different age $a \in\{15,20,25,30,35,40\}$. Different levels of competition $h$ are indicated by different colours. The level of cross-protection was $\theta_{n v t}=40 \%$. 

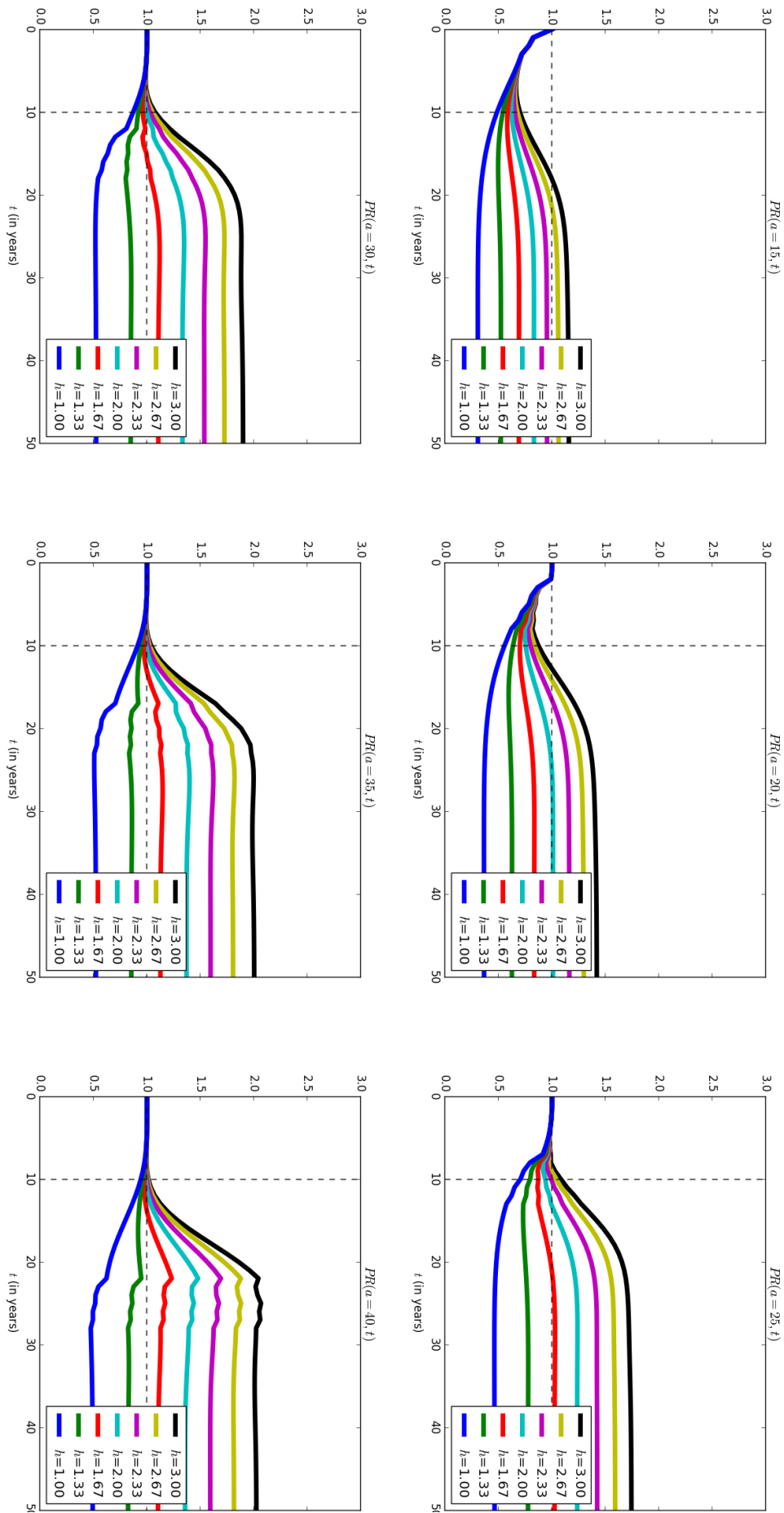

Figure S6.27: (Sensitivity analysis B) Prevalence ratio $P R(a, t)$ over time since the introduction of vaccination evaluating short-term type replacement. Each panel shows $P R(a, t)$ for a different age $a \in\{15,20,25,30,35,40\}$. Different levels of competition $h$ are indicated by different colours. The level of cross-protection was $\theta_{n v t}=40 \%$. 

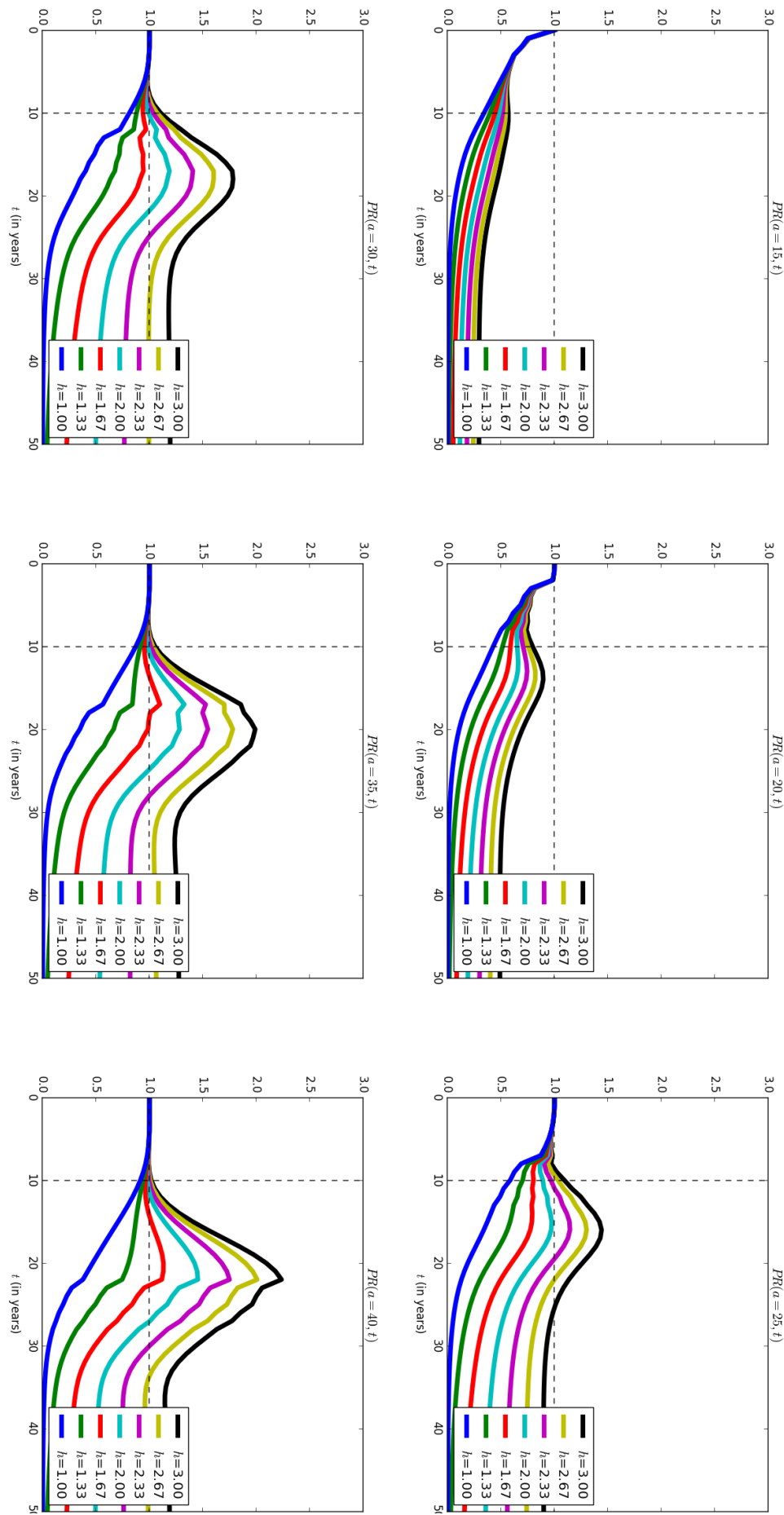

Figure S6.28: (Sensitivity analysis $\mathbf{C}$ ) Prevalence ratio $P R(a, t)$ over time since the introduction of vaccination evaluating short-term type replacement. Each panel shows $P R(a, t)$ for a different age $a \in\{15,20,25,30,35,40\}$. Different levels of competition $h$ are indicated by different colours. The level of cross-protection was $\theta_{n v t}=40 \%$. 

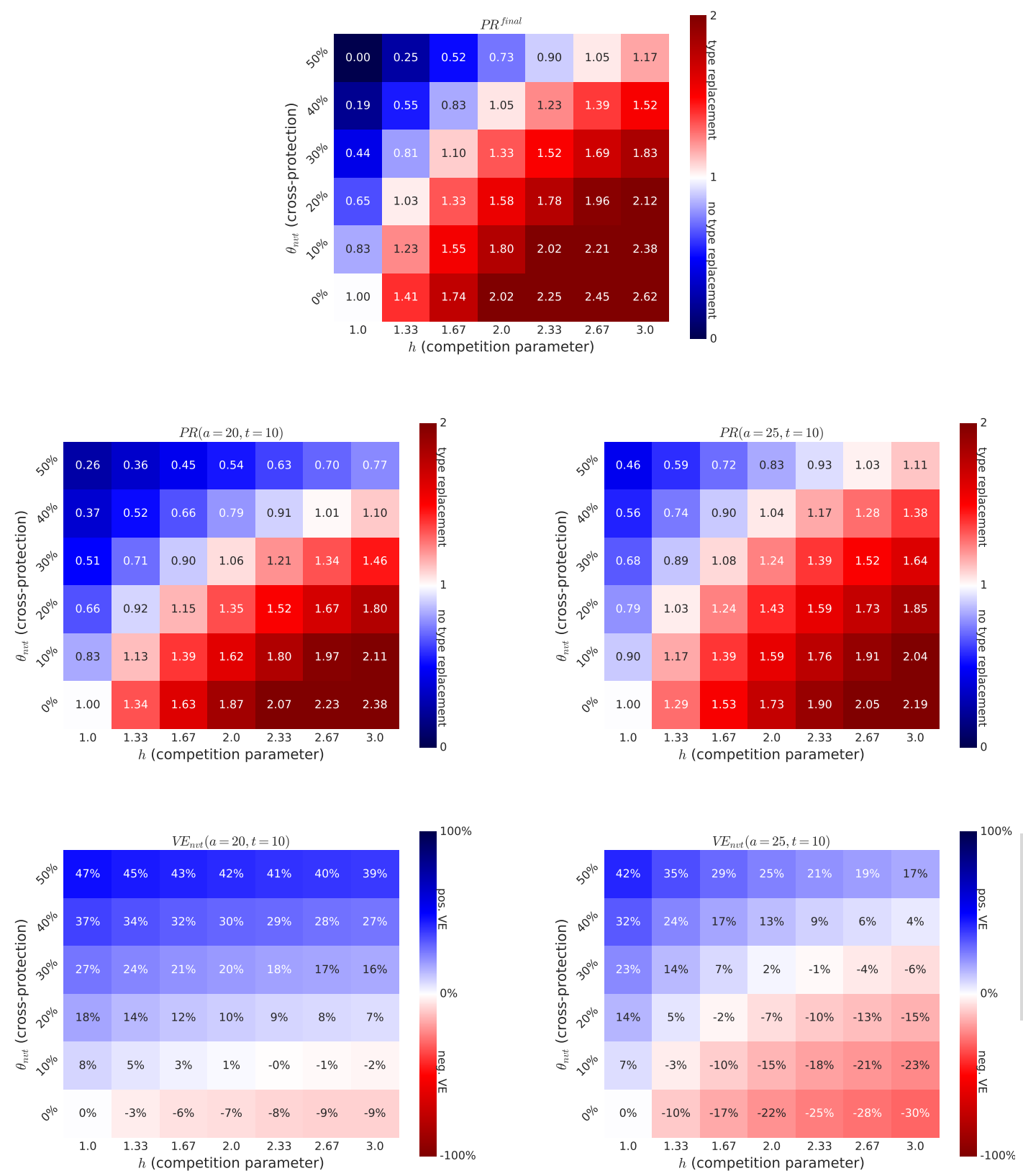

Figure S6.29: (Sensitivity analysis A) Top panel: Prevalence ratio $P R^{f i n a l}$ evaluating final type replacement for different levels of competition $h$ and cross-protection $\theta_{n v t}$. Red (blue) corresponds to the (non-)occurrence of final type replacement. Middle row: Prevalence ratio $P R(a, t)$ evaluating short-term type-replacement among (left) 20-year-old and (right) 25-year-old women at 10 years after vaccination. Bottom row: Vaccine effectiveness for the non-vaccine type $V E_{n v t}(a, t)$ evaluated among (left) 20-year-old and (right) 25-year-old women at 10 years after vaccination. In the middle and bottom rows, red (blue) corresponds to (no) indication of type replacement based on the applied measure. 

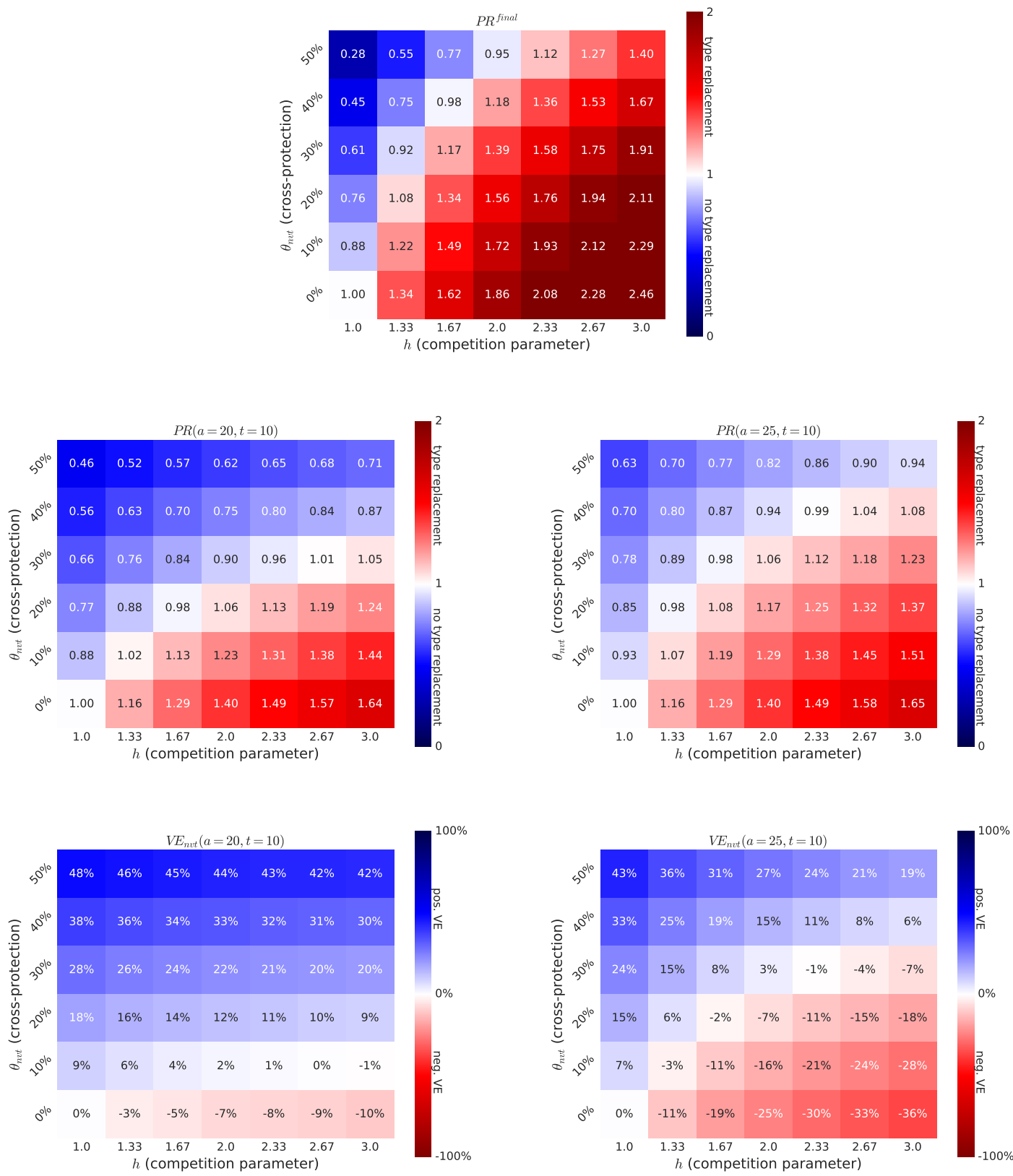

Figure S6.30: (Sensitivity analysis B) Top panel: Prevalence ratio $P R^{\text {final }}$ evaluating final type replacement for different levels of competition $h$ and cross-protection $\theta_{n v t}$. Red (blue) corresponds to the (non-)occurrence of final type replacement. Middle row: Prevalence ratio $P R(a, t)$ evaluating short-term type-replacement among (left) 20-year-old and (right) 25-year-old women at 10 years after vaccination. Bottom row: Vaccine effectiveness for the non-vaccine type $V E_{n v t}(a, t)$ evaluated among (left) 20-year-old and (right) 25-year-old women at 10 years after vaccination. In the middle and bottom rows, red (blue) corresponds to (no) indication of type replacement based on the applied measure. 

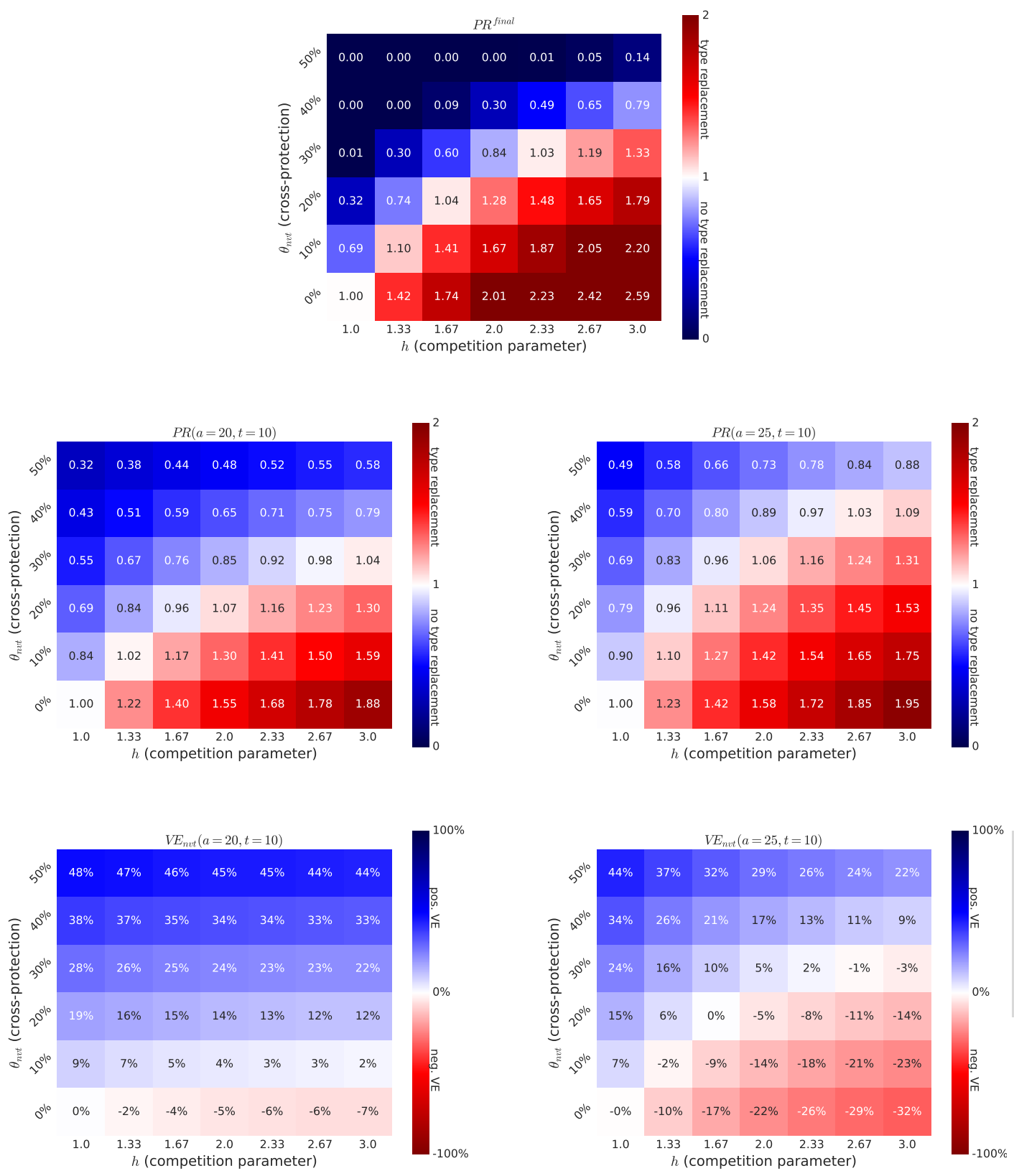

Figure S6.31: (Sensitivity analysis C) Top panel: Prevalence ratio $P R^{\text {final }}$ evaluating final type replacement for different levels of competition $h$ and cross-protection $\theta_{n v t}$. Red (blue) corresponds to the (non-)occurrence of final type replacement. Middle row: Prevalence ratio $P R(a, t)$ evaluating short-term type-replacement among (left) 20-year-old and (right) 25-year-old women at 10 years after vaccination. Bottom row: Vaccine effectiveness for the non-vaccine type $V E_{n v t}(a, t)$ evaluated among (left) 20-year-old and (right) 25-year-old women at 10 years after vaccination. In the middle and bottom rows, red (blue) corresponds to (no) indication of type replacement based on the applied measure. 

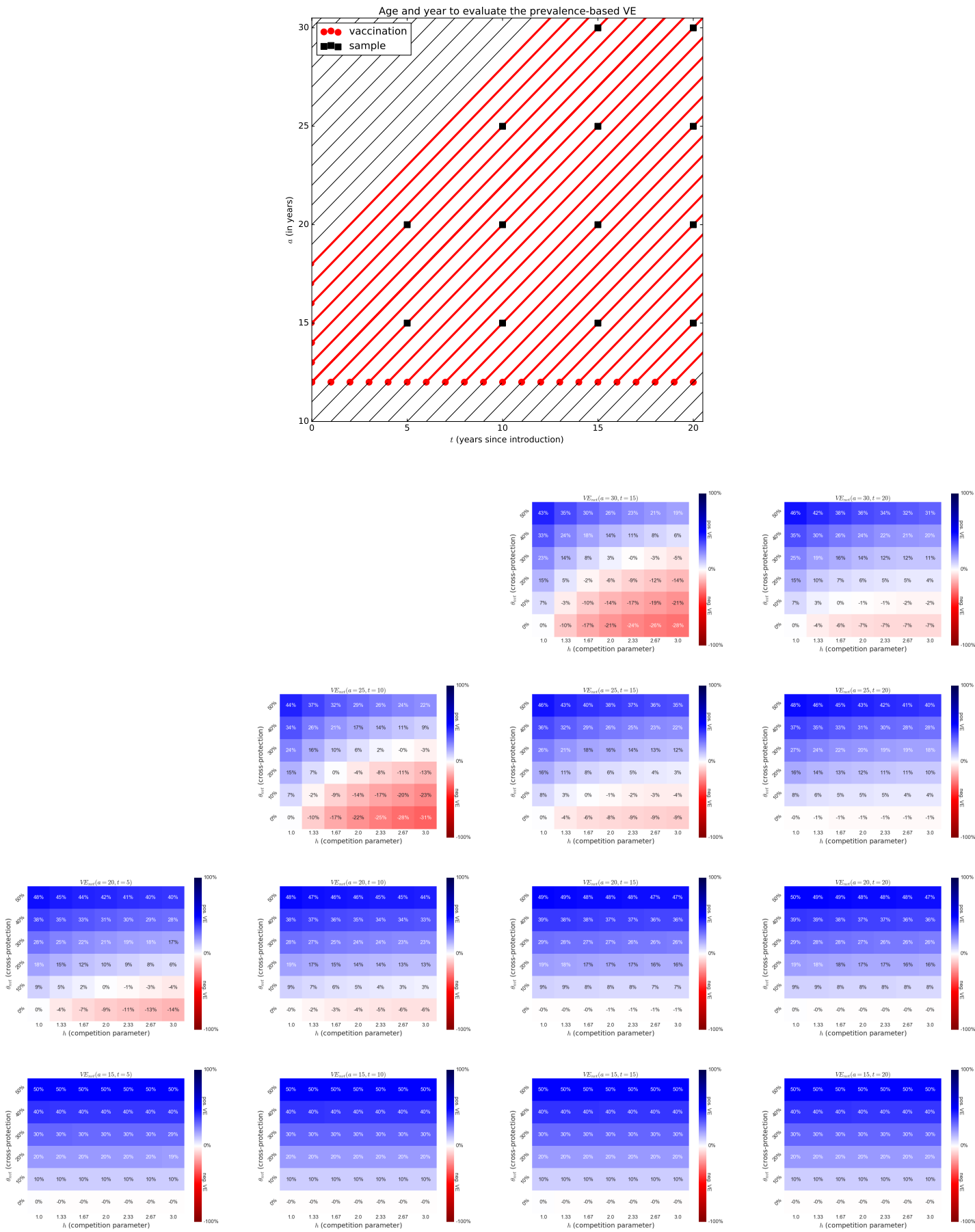

Figure S6.32: Vaccine effectiveness for the non-vaccine type evaluated among different ages $a$ and different time points $t$ since introduction of vaccination as indicated by the black squares in the Lexis diagram at the top. 
Chapter 7

General discussion 
Many pathogen species are characterised by multiple strains. If the different strains of a pathogen interact, their transmission and disease dynamics are no longer independent, and an intervention upon one strain could lead to changes in the occurrence of other strains. In this thesis, we studied whether and how vaccination against a subset of strains of a human pathogen would affect the occurrence of the non-targeted strains of the pathogen. To do so, we evaluated and built on existing methodologies to address the following three problems: 1 . inference of between-strain interaction from pre-vaccination co-occurrence data; 2. prediction of strain replacement using inferred information on between-strain interaction; 3. evaluation of strain replacement using post-vaccination data. We have investigated the usefulness and mechanistic interpretation of several existing methods, and thus generated a deeper understanding of their theoretical basis. Furthermore, we have brought to light the limitations of some methodologies and provide solutions to some of those (i.e. limitations identified).

In this chapter, we first summarise and discuss our methodological findings. We then briefly discuss the role of mathematical modelling in this thesis. Subsequently, we turn from theory to practical applications and discuss the implications of our findings for real-world multi-strain pathogens, with a special focus on Streptococcus pneumoniae (pneumococcus) and the human papillomavirus (HPV). Finally, we conclude with some remarks concerning the public health perspective.

\section{Inference of between-strain interaction}

Understanding how different strains of a pathogen interact is an essential step towards understanding and predicting the impact of vaccination on non-targeted strains. Aspects of between-strain interactions that could be relevant for the outcome of vaccination include whether, to what extent, and through which modes the pathogen strains interact. In Chapters 2-4, we studied various methods for inferring these aspects of between-strain interactions from longitudinal or crosssectional data of pathogen strain co-occurrence in human hosts.

This section starts with discussing our findings regarding longitudinal data and cross-sectional data. We then discuss different modes of interaction, heterogeneity in interaction across strains and the ways in which interactions between different strains combine. Finally, we examine the impact of confounding due to common risk factors for different strains.

\section{Longitudinal data}

Longitudinal data, which are generally considered necessary for inferring causal relationships [1, 2], have also proven to be valuable for the inference of betweenstrain interaction. By recording the (co-)occurrences of different strains over time, 
longitudinal data provide information on how pathogen strains affect one another at different stages of their natural history (e.g. acquisition, colonisation, infection, clearance, and immunity).

Longitudinal data are useful for inferring information about various modes of interaction. We have shown that longitudinal co-occurrence data are particularly helpful for inferring the mode of "direct" interactions in acquisition and clearance, i.e. when colonisation or infection of one strain modifies the hazards of acquisition or clearance of another strain (Chapters 2 and 3). Based on longitudinal data, these two modes of interaction can be quantified in terms of hazard ratios (HRs). These HRs compare the hazards of acquiring or clearing a given strain between individuals with and without colonisation (or infection) of other strains. Whether, to what extent, and by what nature (i.e. competitively or synergistically) pathogen strains interact can then be inferred by inspecting whether, to what extent, and in which direction the estimated HRs diverge from unity.

Nevertheless, as longitudinal data contain rich information about strain cooccurrence over time, the estimation of the defined association measures for interactions from such data might impose a high computational burden (see Table 7.1 for an overview of comparative advantages between longitudinal and cross-sectional data for the inference of between-strain interaction). Multi-state models provide a natural framework to estimate interactions between multiple strains [3-6] but can be computationally expensive, especially when many distinct combinations of co-occurring strains are observed in the data. Exploring the possible number and timing of acquisition and clearance events between the discrete sampling time points then becomes a combinatorically complex task. Previously developed multi-state models used to estimate interactions between serotypes of pneumococcus were only able to cope with data recording simultaneous co-occurrence for up to two strains. However, due to advances in molecular detection methods, co-occurrences of more than two serotypes are more often found in carriage data. To accommodate analyses on such data, we developed a novel estimation method that can cope with higher numbers of co-occurring strains (Chapter 2). The computational burden was tackled by approximating the likelihood function with only those trajectories between the sampling time points that consist of a minimum number of acquisition and clearance events. Using simulated data, we demonstrated satisfactory performance by the proposed method for inferring direct interactions in acquisition and clearance. A point of caution is that long intervals between sampling time points (in relation to the time scale of acquisition and clearance events) may lead to biases in the estimates of between-strain interaction (Chapter 2).

By developing the above estimation method, we have enabled inference of between-strain interaction from longitudinal data containing observations of simultaneous co-occurrence of many strains within the multi-state framework. It should be noted that this was already possible using marginal approaches. In these ap- 
Table 7.1: An overview of comparative advantages between longitudinal and crosssectional data for the inference of between-strain interaction. ${ }^{*}$ Control for confounding due to common risk factors is also possible in the cross-sectional setting but may not perform as well as in the longitudinal setting. ${ }^{\dagger}$ A large sample size would likely in turn facilitate the items listed before it.

\begin{tabular}{l||cc}
\hline & Longitudinal & Cross-sectional \\
\hline Disentangle multiple modes of interactions & $\checkmark$ & \\
Infer competition in cross-immunity & $\checkmark$ & \\
Infer how interactions between strains combine & $\checkmark$ & \\
Control for confounding due to common risk factors & $\checkmark$ & $\checkmark^{*}$ \\
Large sample size & & $\checkmark^{\dagger}$ \\
Computational ease & & $\checkmark$ \\
\hline
\end{tabular}

proaches, estimation of interactions corresponding to different sets of strains (e.g. pairs of strains) or different modes (e.g. in acquisition or clearance) is performed separately [7-9]. By doing so, marginal approaches can more easily cope with data with multiple strain co-occurrence and are less computationally intensive. However, these approaches do not take into account the sequence in which acquisition and clearance events of different strains occur, nor do they naturally control for multiple testing, with the risk of biased results. Therefore, although marginal methods may be convenient in the exploration phase, non-marginal methods, such as multi-state models, are preferable for formal analyses.

\section{Cross-sectional data}

While longitudinal data are in general considered to be more powerful in studying complex processes, such as between-strain interaction, cross-sectional data offer other advantages, e.g. convenience of data collection and the ability to easily achieving large sample sizes (Table 7.1). In our work, we demonstrate that crosssectional data are also useful for the inference of some aspects of between-strain interactions [1].

In Chapters 3 and 4, we showed that cross-sectional data are useful for inferring the modes of direct interactions in acquisition and clearance, provided that the data are sampled at the system's steady state. Specifically, we showed that (at the steady state) the HRs indicating direct interaction in acquisition or clearance can be estimated in the form of the odds ratio (OR) of co-occurrence, which is the OR comparing the occurrence of one strain between individuals with and without the occurrence of another strain.

Nevertheless, due to its "snapshot" nature, cross-sectional data are inherently limited in distinguishing between different modes of interactions (Table 7.1). In the context of inferring between-strain interactions in the modes of direct interaction, the OR of co-occurrence cannot distinguish between direct interaction in acquisition and clearance. A finding of OR indicating negative association may reflect 
either competition in acquisition or competition in clearance. In fact, a finding of negative association may even reflect a comparably stronger competition in one mode than the other, although this possibility seems less realistic.

Opportunely, our analysis shows that the cross-sectional OR does correspond to a useful summarizing quantity of these two modes of interactions (Chapters 3 and 4). The summarizing quantity is the ratio of the two HRs, i.e. the HR indicating direct interaction in acquisition divided by the HR indicating direct interaction in clearance. In a previous study considering at most two co-colonizing serotypes of pneumococcus, this cross ratio has been interpreted as "the relative reduction in the expected time spent doubly colonised per time unit than spent singly colonised with a given strain, compared with no competition" [6]. We moreover showed that this cross ratio is better identifiable from data than the separate HRs (Chapter 2). Further to this, this cross ratio has a key role in determining the outcome of strain replacement (Chapter 3). Hence, there is value in using cross-sectional data for the inference of between-strain interaction as well (Table 7.1).

Nevertheless, as correspondence is only guaranteed in the system's steady state, one should be careful in selecting the appropriate time to conduct a crosssectional study of between-strain interaction. For example, in the transient phase soon after the introduction of vaccination, while the system is rebalancing into a new steady state, the co-occurrence patterns of the pathogen strains may be less informative of the between-strain interaction. Possibly, the correspondence is still be good enough if the system is close enough to the steady state. Further investigation is needed to quantify the usefulness of cross-sectional data when not sampled in the system's steady state.

Notably, the convenient correspondence between the considered longitudinal and cross-sectional quantities is grounded in the more general mathematical concept of "reversibility" in dynamical systems. For a dynamical system to be in its steady state, many different quantities need to be in balance for each of them to remain constant. In this application of inferring between-strain interactions, the balancing quantities are the prevalence of co-occurrence states that appear in the OR and the hazards that appear in the HRs (Chapter 3). There are more applications in which a convenient correspondence holds between the relevant cross-sectional and longitudinal quantities [1, 10]. One closely related application (which has inspired the findings regarding reversibility in this thesis) is the correspondence between cross-sectional and longitudinal estimates of the efficacy of polyvalent vaccines amidst between-strain interactions [11].

\section{Different modes of between-strain interaction}

Our analysis demonstrated that information on direct interaction in acquisition and clearance can be inferred from longitudinal data and, to a certain extent, also from cross-sectional data. However, pathogen strains may also interact through 
other modes. Some other modes of interaction can also be inferred from the form of longitudinal or cross-sectional data we considered, while some other cannot, or only when certain additional information is available. Here, we discuss the inference of several important alternative modes of interaction.

An important mode of interaction that we have also studied in this thesis is competition through cross-immunity, i.e. occurrence of one pathogen strain, upon immune clearance, inducing protection against other strains. Similarly to direct interactions, cross-immunity can also manifest through inhibition of acquisition or enhancement of clearance of other strains. Previous studies have highlighted that the inference of cross-immunity from cross-sectional data, recording only the current status of pathogen strain occurrence, could be problematic, as these data do not necessarily provide information about previous episodes of occurrence and hence the immune status of the examined individuals. When inference is based on the cross-sectional OR of co-occurrence, the presence of cross-immunity may induce positive association and lead to erroneous conclusions of synergistic interaction (Table 7.1) [12, 13].

In Chapter 3, we characterised in more detail how such reverse correspondence arises mechanistically. The reasoning can be illustrated using an example with two strains $(A$ and $B$ ) by considering how the risk of acquiring strain $A$ is modified by the presence of strain $B$. The cross-sectional OR of co-occurrence would correctly indicate competition if the risk of acquiring strain $A$ is lower in the individuals currently infected with strain $B$ than in those that are uninfected with strain B. However, this risk (of being infected with strain A) is actually lower in the latter group, as it also includes those individuals that were previously infected with strain $B$ and hence have cross-immunity for strain A. As a result, cross-immunity reversely leads to a positive association in the OR.

If we would like to infer information about cross-immunity (e.g. whether and to what extent strains compete through this mode) from cross-sectional data, an adequately defined measure should instead compare individuals with and without immunity. Nevertheless, such a measure can only be defined if there are additional data on the immune status of the study participants (e.g. serological data), which is not always possible. In addition, there may not always be enough prior knowledge about whether direct interaction or interaction through cross-immunity (or both) is present, in which case it is hard to judge which definition of the OR is more appropriate. Hence, when using cross-sectional data, one should be particularly careful about the possible ambiguity due to the presence of cross-immunity and reflect upon whether the defined association measure is suitable.

By comparison, it is more feasible to infer interaction through cross-immunity from longitudinal data. It is possible to estimate HRs corresponding to direct interactions as well as HRs corresponding to cross-immunity. In a multi-state model, for instance, this can be achieved by expanding the model state space with states 
of immunity and subsequently defining HRs that compare the hazards of strainspecific acquisition or clearance across individuals with and without immunity. If explicit information about the individuals' immune status is available (e.g. based on serology), estimation of such HRs is relatively straightforward. Even if such information is not available, it might be possible to derive the immune status based on the time since previous clearance events. Hence, the longitudinal setting is more useful for inferring competition through cross-immunity.

There are modes of between-strain interactions not considered in this thesis. For instance, pathogen strains can also interact by enhancing or suppressing the extent of colonisation or infection of one another [14, 15]. Inference of such modes of interaction is more feasible using quantitative data indicating the extent of colonisation or infection, e.g. data of bacterial or viral loads, than based on data that indicate the presence or absence of colonisation or infection. Nevertheless, the extent of occurrence is likely to influence the timing of acquisition and clearance events. For example, when infection with one strain is suppressed by another strain, the suppressed strain will likely be cleared earlier. Hence, presence/absence data may be sufficient to infer the existence of such modes of interaction. However, in case one would like to characterise how within-host dynamics of different pathogen strains interact in more detail, quantitative data remain necessary.

Finally, there might be some modes of between-strain interactions that cannot be inferred from the forms of longitudinal and cross-sectional data we considered in this thesis. Competition due to a limited capacity of transmission is one such example. Such a mode of competition occurs if, for example, the co-infecting strains have to enter a limited amount of body fluids (e.g. blood, saliva) during transmission. This mode of competition is different from the type of within-host interaction discussed in the previous paragraph, since competition in the capacity for transmission does not necessarily imply competition in within-host dynamics. To infer this mode of competition, the probability of transmission needs to be compared between individuals carrying a single strain and individuals carrying multiple strains. To estimate such a probability, the forms of longitudinal and cross-sectional data we considered are not suitable. Instead, we need data on individuals between whom transmission may occur, e.g. clustered data of individuals within households or partnerships.

In view of the different modes of interaction possible, when inferring betweenstrain interaction from an existing dataset or planning a new study, it is important to reflect whether the hypothesised modes of interaction match with the modes that can be inferred from the available data and the defined measures of association. Furthermore, when interpreting estimates of association measures, it is important to acknowledge and discuss the possible ambiguity and limitations. 


\section{Heterogeneity of between-strain interaction}

There are other aspects of between-strain interactions relevant for the outcome of vaccination that are also worth inferring. For pathogens of which the strains are known to interact, it could also be useful to investigate whether and to what extent interactions differ across strains in strength or even by nature (e.g. competitively versus synergistically). If interactions are indeed heterogeneous, vaccination may have differential impacts on the different non-targeted strains. Knowing which pathogen strains have a competitive advantage may help us identify which strains would increase most in strain replacement.

In Chapter 4, we explored the utility of network inference models to infer heterogeneous strengths of interaction between pairs of pathogen strains from crosssectional data. We found good performance under a simulation setting in which strains were assumed to interact through the modes of direct interaction in acquisition and clearance. Moreover, the underlying parameters of interaction were recovered without bias, again in the form of the cross hazard ratio (regarding interaction in acquisition and clearance). Although not considered in this thesis, some previous studies utilizing multi-state models have implemented estimation of heterogeneous interactions by defining strain-specific interaction parameters (3. 5.

Again, if strains are likely to compete through cross-immunity, inference of interaction based on cross-sectional data is not suitable due to the reverse correspondence (mentioned in the previous section), hence also not suitable for inferring heterogeneous interactions. Inference using longitudinal data is more suitable for this purpose and has, for instance, been done for pneumococcus and HPV [7],9 16].

Besides using the aforementioned non-marginal approaches, marginal approaches have also been used to estimate interactions between different sets of strains (e.g. pairs of strains). However, these approaches may be more prone to bias [7, 9, 16]. It is conceivable that indirect interactions via other strains are particularly disruptive for marginal approaches when interactions are heterogeneous. Again, estimation of interactions between many sets of strains using marginal approaches, without correction for multiple testing, may lead to false discovery of non-existent interactions. Hence, it is good practice to verify results from marginal approaches using non-marginal approaches.

Finally, while inference of heterogeneous interactions may be interesting and relevant, there are some limitations. The sample size of a given dataset may not allow estimation of the associated high number of interaction parameters (as was the case for our analysis in Chapter 2). To illustrate, with 15 strains, which is the order of magnitude of the number of high-risk HPV types and the number of pneumococcal serotypes in carriage studies, there are already 105 pairwise combinations. There are some ways to reduce the number of interaction parameters. 
For instance, one could group strains based on their biological characteristics (e.g. membership to clades and serogroups), and assume the same interaction parameter, or distribution for different interaction parameters, for each group.

\section{Combining interactions between different strains}

Another interesting but less frequently considered aspect of between-strain interactions is how multiple strains combine their interactions towards another strain [17]. For example, when multiple strains co-occur in the same host, there are conceivably different ways in which their interactions act together to inhibit the acquisition of an additional strain.

In this thesis, we have explored two possible ways in which interactions of different strains combine in the mode of acquisition as well as clearance during co-occurrence. The first one was the multiplicative rule, according to which each strain occurring in the host contributes a multiplicative factor to the total strength of interaction (Chapters 4 and 5). In the mode of acquisition, for instance, the hazard of acquiring an additional strain is then modified by a ratio equal to the product of all strain-specific contributing factors. The second one is the "all-or-nothing" rule, according to which additional strains do not induce more interaction after the first one has been acquired (Chapters 2 and 4).

Similarly, there are different ways whereby cross-immunity, conferred by multiple previous episodes of carriage or infection with different strains, accumulates as individuals age [18, 19]. For instance, according to a multiplicative rule, the hazard of acquiring a new strain might diminish exponentially with each additional episode. While according to an all-or-nothing rule, after the first episode, subsequent episodes no longer diminish the acquisition hazard. In fact, other intermediate or more extreme rules are possible.

The way in which interactions between multiple strains combine has often been chosen for the convenience of modelling or parameter estimation. For instance, the multiplicative rule matches well with network inference models (Chapter 4), and the all-or-nothing rule is useful for limiting the number of interaction parameters. We know of little clear empirical evidence supporting or disproving the different alternatives. Yet, when assumptions about how interactions combine are incorrect, the estimates of interactions may become biased, and it is unclear what the estimated parameters actually represent. In turn, incorrect assumptions about how interactions combine may influence the validity of predictions of strain replacement (Chapter 5).

More research is needed to determine the feasibility of identifying how interactions between strains combine for real-world pathogens. Longitudinal data and data across age groups may be more useful for this purpose than cross-sectional data, as they provide information of the sequence of occurrence of different strains 
(Table 7.1). Possibly, knowledge about how interactions combine can be inferred by constructing different models (e.g. multi-state models) with alternative assumptions and subsequently comparing the corresponding goodness-of-fit. In addition, modelling studies may improve the understanding of how these assumptions influence predictions of strain replacement.

\section{Confounding due to common risk factors}

In this thesis, we have also investigated how heterogeneity in risk factors for acquiring different strains across hosts may confound estimates of between-strain interactions and how to cope with them. In general, confounders could bias the effect of interest in both the direction of positive as well as negative associations. However, in the context of inferring between-strain interactions, confounders are mostly expected to induce a bias towards positive associations [13, 20, 21]. The intuition behind this is that a risk factor (e.g. being immunosuppressed or having many contacts relevant for the spread of the pathogen) that increases the risk of one strain is also expected to increase the risk of other strains. In Chapter 3 , we formally derived this direction of bias based on algebraic arguments and so provide a more solid understanding of this underlying intuition.

Knowing the direction of bias is useful, as it helps to derive the true effect of between-strain interaction. Under a bias towards positive associations, a finding of negative association would likely reflect true competition. Conversely, a finding of positive association could result from confounding by common risk factors while the underlying interaction is competitive. Standard statistical techniques exist to adjust for possible bias due to common risk factors for both the cross-sectional and longitudinal settings. In this thesis, we have demonstrated for some of these techniques that they also work well in the context of inferring between-strain interaction. When common risk factors are observed and measured, they could be included as independent variables that act on the probability or hazard of the occurrence across all strains. This approach can also be applied to network inference models, as we have demonstrated (Chapter 4). When common risk factors are not observed, individual-specific risk levels could be modelled as random effects that are common for all strains within each individual [20], which we also have demonstrated for the multi-state framework (Chapter 2).

Although statistical adjustment for unobserved common risk factors is possible through the modelling of random effects, there is still value to model them explicitly, if possible. How well adjustment for common risk factors works depends on other things such as how well the distribution of the modelled effects is specified and how strongly the unobserved risk factors correlate with the observed risk factors included in the analysis. In general, it is difficult to judge whether unobserved common risk factors are over- or under-adjusted for and so whether estimates of between-strain interactions are over- or under-estimated. Hence, it remains 
paramount to collect data on possible confounders and model them whenever possible.

Finally, the effects of both observed and unobserved common risk factors are more easily identifiable in the longitudinal setting (Table 7.1), as there are multiple observations per individual at different time points to characterise the individuals' risk [22]. Yet, even in the cross-sectional setting, there are multiple observations per individual, which are the multiple observations concerning the different strains. Moreover, cross-sectional data usually achieve a higher sample size, which also facilitates the identification of the individuals' risk and hence between-strain interaction. Future research may help to better understand the comparative advantages between the longitudinal and cross-sectional designs for the purpose of inferring between-strain interaction.

\section{Prediction of strain replacement}

In this thesis, the second methodological problem we addressed was how to use inferred information on between-strain interaction to predict the occurrence of strain replacement. In a way, the problems of inference and prediction are the inverse of one another. While the inference problem involves specifying and teasing apart different aspects of how pathogen strains interact, the prediction problem contends with summarizing the inferred information on different aspects into one outcome. Given the complex ways in which pathogen strains may interact, e.g. through different modes simultaneously and to different extents between different strains, it is not straightforward how all of these aspects work together to determine the outcome of vaccination.

\section{A novel prediction framework}

In Chapter 5, we attempted to tackle the prediction problem in a setting where pathogen strains may interact heterogeneously in strength and nature, and through direct interactions in acquisition and clearance. To do this, we developed a novel framework that can be used with information on between-strain interaction, inferred from either cross-sectional or longitudinal presence/absence data. The framework provides predictions of whether vaccination against a subset of strains would increase, decrease, or have no effect on the prevalence of the non-targeted strains. It provides predictions for individual non-targeted strains as well as for groups of non-targeted strains. As such, it may help to evaluate whether vaccination is desirable overall and to identify potential strains that would dominate after strain replacement. Although we did not explore the possibility, the numerical values of the predictors may also be informative for the quantitative change in the occurrence of non-targeted strains. 
The rationale behind the construction of the strain-specific predictors was to simply approximate the effect of vaccination based on a risk ratio that compares the occurrence of a given non-targeted strain in the presence versus absence of any of the vaccine-targeted strains in the data. Group-specific predictors are subsequently obtained by pooling the strain-specific predictors belonging to the group. Furthermore, possible simultaneous interaction through direct interaction in acquisition and clearance is summarised through the cross HR of acquisition and clearance and the corresponding $\mathrm{OR}$, which we have shown to have predictive value for strain replacement (Chapter 3).

In a simulation study, we demonstrated satisfactory performance by the proposed prediction framework. Although the proposed prediction framework can be applied regardless of how interactions of different strains combine, it performs better under a multiplicative than an all-or-nothing rule. Furthermore, we found that prediction could be challenging when the indirect impact of vaccination via other non-targeted strains is strong. This demonstrates the difficulty of trying to predict an outcome in a complex and dynamical system with only simple summary quantities computed from one dataset.

If the pathogen strains interact through modes other than direct interaction, the proposed framework would need to be adapted. This could possibly be achieved by redefining the predictors using risk ratios that capture the relevant modes of interaction. Further research is needed to investigate whether such adaptation is possible and whether the performance of the framework would remain satisfactory.

\section{Prediction based on dynamical models}

Given the complex and dynamical way in which pathogen strains may interact, prediction based on dynamical models may provide an attractive alternative [23. 26]. In a dynamical model, inferred information regarding between-strain interactions is incorporated as ranges or values for the model parameters. Whether strain replacement is predicted or not then follows as an emergent property of the model. An advantage of such an approach is that the model could easily incorporate different elements about between-strain interaction as well as other elements relevant to the transmission dynamics, e.g. heterogeneity of the host population, within-host strain dynamics, and vaccine-induced cross-protection [17, 27, 28]. A caveat, however, is that modelling of additional elements would require additional data or assumptions. This may introduce additional uncertainty or ambiguity to the resulting prediction. A complex and all-encompassing model could be difficult to parametrise and computationally intensive, with the risk of becoming a black box.

Arguably the impact of vaccination on the non-targeted strains is mainly driven by a few key elements, in which case, capturing these elements would be sufficient to provide a reasonable prediction. In that sense, there are advantages to a 
simpler and more easily interpretable framework, such as ours. It seems that, in the end, the crucial step is to obtain a solid understanding of how the pathogen strains interact, from which reasonable prediction of strain replacement could be derived, based on multiple alternative approaches.

\section{Propagation of bias}

Finally, we note that if the inferred information on between-strain interactions is biased by confounding due to common risk factors, such bias could in turn influence the prediction of strain replacement. Due to the direction of bias towards positive associations, we would expect the bias to mainly lower the sensitivity of predicting strain replacement while being less disruptive for the specificity. In other words, prediction of strain replacement is likely to remain true, while prediction of a lack of strain replacement may become less reliable. Additional simulation studies may help to confirm this intuition. To avoid propagation of bias, possible confounding should already be addressed in the inference step, for example, based on the methodologies that we have discussed in the inference section.

\section{Evaluation of strain replacement}

As the last methodological problem, we considered the evaluation of strain replacement using post-vaccination data. In chapter 6 , we examined the validity of two commonly used effect measures, computed from two forms of postvaccination data, for evaluating strain replacement. The first effect measure is the prevalence ratio $(\mathrm{PR})$ comparing the prevalence of non-targeted strains between the pre- and post-vaccination eras, hence capturing the trend of non-targetedstrain occurrence over time [29, 30]. The second one is vaccine effectiveness (VE), which compares the risk of non-targeted strains between vaccinated individuals and unvaccinated controls [11, 31].

\section{Trend of non-targeted-strain occurrence}

Regarding the use of the PR in trend data, our modelling findings affirm its validity for evaluating strain replacement. Nevertheless, there are a few points of caution. First, evaluation based on the PR may not be sensitive in indicating strain replacement in the first period right after introducing vaccination. One obvious reason is that it may take time for the non-targeted strain to rise in prevalence. In Chapter 6 , we showed that there is an additional reason why evaluation soon after introduction could be problematic. In a transmission model, we illustrated the possibility that the prevalence of a non-targeted strain might initially decrease after the introduction of vaccination before rebounding into strain replacement. This occurs 
when the vaccine also induces cross-protection for the non-targeted strain, but the protection is not strong enough to completely prevent strain replacement. Due to the cross-protection, immediately after the start of vaccination, there is additional protection for the non-targeted strain besides the natural cross-immunity induced by the circulation of the vaccine-targeted strains. Of note, this phenomenon of initial decrease preceding strain replacement is analogous to the concept of a vaccination-induced honeymoon period, already known in the context of a single infectious agent. It describes the initial period of exceedingly low occurrence of the infectious agent immediately after the introduction of vaccination due to the combined protection from the vaccine and natural immunity [32, 33].

To judge when a follow-up period is long enough for evaluation based on trend data, it is helpful to consider the dynamical nature of multi-strain transmission. In non-linear dynamical systems, upon perturbation, the state of the system does not necessarily have to evolve monotonically while converging towards a new steady state. To understand the temporal behaviour of the system, it is important to characterise time scales over which different balancing forces act. On one hand, vaccine-induced cross-protection for the non-targeted strains is immediately effective upon the introduction of vaccination. On the other hand, the emergence of strain replacement might require more time, as it requires substantial reduction of the circulation of the vaccine-targeted strains first. The time needed to achieve a substantial reduction in the vaccine-strain circulation depends on how fast the population becomes vaccinated and the extent of herd effects in the unvaccinated part of the population. Presumably, transmission through sexual contact with a relatively low rate of acquiring new sexual partners and high assortativeness in age and risk groups may entail a longer time for reduction of vaccine strains as compared to close-proximity transmission with a relatively high rate of contact. In addition, the time scale over which strain replacement occurs depends on the time scale associated with the natural history and the mode of competition. For example, competition through long-lasting natural cross-immunity might also require more time to give rise to competitive release than direct competition.

Another point of attention for evaluation based on the trend of non-targeted strains is the possible differential occurrence of strain replacement across different subpopulations (e.g. different age groups and risk groups) [34, 35]. For instance, if strain replacement occurs in the general population but not in the subpopulation where the trend of non-targeted strains is evaluated, strain replacement may remain unobserved. In Chapter 6, we found, in a model calibrated for HPV transmission, that strain replacement could occur in older age groups while not in younger age groups. In addition, changing contact behaviour (relevant to pathogen spread) in the population may also obscure the evaluation of strain replacement [36, 37]. To distinguish between the possible occurrence of strain replacement and other secular trends, additional data on contact behaviour over time are essential. In summary, when interpreting a given trend of non-targeted strains for the evalua- 
tion of strain replacement, it is important to take into account the different internal and external factors concerning the transmission and disease dynamics.

\section{Vaccine effectiveness for non-targeted strains}

Besides through trend data, evaluation of strain replacement has also been done based on vaccine effectiveness (VE) for the non-targeted strains, i.e. the ratio comparing the risk of carriage or infection of non-targeted strains between vaccinated and unvaccinated individuals [31, 38, 39]. The underlying intuition of such an evaluation is that, if between-strain competition is present, the reduced occurrence of the vaccine-targeted strains in vaccinated individuals would increase their susceptibility to non-targeted strains. Hence, a negative VE is interpreted as a sign for strain replacement and a positive VE as the lack of it.

In Chapter 6, we found that, if vaccine-induced cross-protection is present, evaluation of strain replacement based on VE is more problematic than if it is based on trend data. If vaccine-induced cross-protection is present, it would decrease the risk of non-targeted strains in the vaccinated individuals as compared to unvaccinated individuals. This drives the VE towards a positive value. Even though competitive release increases the risk of non-targeted strains in vaccinated individuals as compared to unvaccinated individuals, the net effect may still be a positive VE while strain replacement occurs at the population level. Furthermore, while evaluation based on trend data should correctly indicate strain replacement if follow-up is long enough, this is not the case for VE (Chapter 6). Hence, evaluation of strain replacement based on the trend of non-targeted-strain occurrence is preferable in the long run.

In addition, our results show that the validity of evaluating strain replacement based on VE also depends on the mode of between-strain interactions and the working mechanism of the vaccine. As for the inference problem, VE is only useful for detecting competitive release if the evaluated risk corresponds well with the underlying mode of between-strain competition. For example, if strains compete through the mode of clearance, a VE based on the incidence of acquisition would not be able to capture the competitive release. Instead, the VE should be based on the incidence of clearance or prevalence. However, if the vaccine-induced cross-protection also works through clearance, VE based on these latter options (i.e. incidence of clearance or prevalence) may still be positive despite the occurrence of strain replacement. This adds to the arguments why evaluation of strain replacement based on the trend of non-targeted-strain occurrence is preferable, and why baseline prevalence studies are essential to enable such an evaluation of strain replacement. 


\section{Usefulness of mathematical modelling}

In various ways, mathematical modelling has helped us to tackle the complex methodological problems around strain replacement in this thesis [40, 41]. Firstly, it has provided a platform on which we can formulate and analyse methods to infer between-strain interaction, and to predict and evaluate strain replacement. For the inference problem, in particular, we have utilised some useful statistical techniques, such as multi-state models, network inference models, and Bayesian inference. In multiple chapters, we have made use of compartmental models, based on either ordinary or partial differential equations, to describe and simulate multi-strain dynamics.

In addition, mathematical modelling has allowed us to investigate the validity of the formulated methods, e.g. effect measures for inferring between-strain interaction and assessing strain replacement. Oftentimes, it is unclear how well the effect that we measure corresponds with the effect we are interested in. Mathematical modelling allows us to validate the correspondence between the two based on simulations or analytical derivations. Repeating such a process of validation under different sets of assumptions moreover allows us to identify conditions under which a given effect measure is valid (or not). If a condition under which a given effect measure is invalid is identified, mathematical modelling may also help us to explore ways to adjust for it.

In the same vein, mathematical modelling allows us to quantify the uncertainty around the predictions by allowing systematic exploration of the elements in the prediction model that we are unsure of. In the context of predicting strain replacement, it is likely that, even with our best efforts, we will not be able to obtain full knowledge of all factors that influence the outcome of vaccination. Instead of only providing one prediction about strain replacement based on the one best-fitting model, it may be more informative to provide a range of predictions generated from a set of plausible models.

Although mathematical models have been praised as useful tools, they have also been criticised as being unrealistic because they come with a set of assumptions. However, making assumptions is an inevitable step when contemplating a complex problem. We take into account elements that we render relevant, disregarding the rest, to better understand the impact of the key elements. Making assumptions in mathematical models does exactly that, but in a more formal framework with less ambiguity. In such a formal framework, we should be better capable to understand the implications of the assumptions made. 


\section{Implications for real-world pathogens}

\section{Streptococcus pneumoniae}

Before the conception of this thesis, it was already clear that pneumococcal conjugate vaccination, targeting a limited number of pneumococcal serotypes, had led to serotype replacement by non-targeted serotypes [30]. Although the total burden of invasive pneumococcal diseases (IPD) has diminished (due to the decrease in some of the most invasive serotypes, e.g. 14, 6B, and 18C), further reduction regarding the burden due to the replacing types (e.g. serotypes $8,9 \mathrm{~N}$, and $15 \mathrm{~A})$ is desirable [35, 42, 43]. A vaccine that effectively protects against all pneumococcal serotypes is considered to be the best option but is not expected to be available in the near future. While such a pan-serotype vaccine is still pending, a viable alternative would be a more "ecologically optimal" vaccine that contains a set of serotypes that would further decrease the disease burden [44, 45]. For the development of such a vaccine, it is necessary to have a good understanding of how pneumococcal strains compete and to know how to use this understanding to predict the impact of any given vaccine candidates.

Using the estimation method we developed in Chapter 2, we analysed a longitudinal dataset of pneumococcal carriage and were able to draw new insights into how pneumococcal serotypes compete during colonisation. In line with previous studies and our expectations, our results indicate the presence of competition between pneumococcal serotypes [3-5, 46, 47]. However, while previous studies mainly indicate competition in the mode of direct competition in acquisition, our result is more in favour of direct competition in clearance. Nevertheless, we expect that, for the purpose of predicting strain replacement, it is sufficient to accurately estimate the combined strength of these two modes of competition. The identification of the exact mode of competition is not necessary, as the combined strength is what eventually determines the occurrence of strain replacement (Chapter 3).

A more surprising finding was the estimated strength of competition, which we estimated to be much weaker than suggested by previous studies. Although we did anticipate weaker competition due to the high sensitivity of detecting cocolonisation in our study, as compared to previous culture-based studies [48-50], the estimates we found were strikingly small. It should be noted that our estimates may have been subjected to some sources of uncertainty, of which the small sample size might be the most influential one. However, if the finding of weak direct competition is correct, this may imply that other modes of competition are more important. In particular, cross-immunity is a mode of competition that has previously been suggested to be important in shaping the ecology of pneumococcal strains [51]. While the existence of cross-immunity is well-supported [52, 53], there are few estimates from epidemiological studies describing the extent of it, or how it may accumulate due to previous episodes of colonisation [5, 9, 54]. In the future, 
analyses of other longitudinal datasets with larger sample sizes, and in somewhat older individuals than we considered, may help to further elucidate the existence of cross-immunity.

Another aspect of interaction we did not explore in the pneumococcal dataset considered is the possible heterogeneity of competitive ability across serotypes. Due to the limited sample size, attempts to estimate strain-specific interaction parameters would probably have resulted in an over-parametrised model prone to overfitting. Some previous longitudinal carriage studies that have considered heterogeneous interactions have shown that strengths of competition in the mode of direct interaction or cross-immunity may differ across pneumococcal serotypes [3, 5, 6, 9, 54]. For instance, serotypes 19F, 6A, and 6B have been reported to have strong competitive abilities. However, these analyses were mainly exploratory and focused on serotypes that are now disappearing due to vaccination, hence further investigation is needed and may require retrospective analyses of previously collected datasets. To this end, cross-sectional data might be helpful for the estimation of heterogeneous interactions in the mode of direct competition (Chapters 3 and 4). However, if cross-immunity is the leading mode of interaction, inference based on longitudinal data would be more suitable (Chapter 3).

Recently, a new aspect about how pneumococcal strains may interact has generated much attention. Specifically, it has been postulated that between-strain interactions may also depend on genetic characteristics beyond those determining the serotype, such as the accessory loci [55, 56]. The underlying hypothesis is that the frequencies of accessory loci of pneumococcus in human populations are subject to some form of negative frequency-dependent selection, inducing interactions between genotypes sharing the same accessory loci. It has been suggested that such selection manifests through the human immune responses [51]; the more common an antigen becomes, the more often it is recognised by the hosts' immune system and the more its fitness reduces. Possibly, inference of such a mode of interaction may also amount to the estimation of certain association measures but then between the occurrence of genotypes. If so, some methodologies considered in this thesis may remain useful.

When new insights on between-strain interaction are generated, it is important to incorporate them into prediction models, e.g. in models built to predict the impact of different vaccine candidates while taking into account strain replacement. For instance, if strains indeed compete heterogeneously (i.e. differing strengths of competition across strains), some serotypes are likely to increase disproportionately in strain replacement. Most prediction models employed so far assume homogeneous competition and are not able to capture such behaviour 445, 57 58]. There are prediction models that incorporate heterogeneous competition and/or cross-immunity, but these are mostly parametrised based on rough estimates due to the lack of empirical data [23-25, 51, 59]. Our relatively simple prediction framework can deal with heterogeneous interaction but requires adap- 
tation to accommodate competition through cross-immunity. Dynamical-modelbased approaches may be a good option if many different and complex details of between-strain interaction need to be incorporated. To assist the construction of prediction models in the future, it would be useful to perform a comparative study on the assumptions of between-strain interaction made by different existing models for predicting pneumococcal replacement.

To conclude, further improvement of pneumococcal vaccines and vaccination strategies necessitates a deeper understanding of between-strain interaction. In our opinion, aspects that require further research include the relative importance of direct competition versus competition through cross-immunity and the existence of heterogeneity in competitive ability across pneumococcal strains. Finally, existing prediction frameworks require further refinement to incorporate a more complete and accurate picture of how pneumococcal strains interact and to account for the uncertainty on aspects that we are (yet) not able to infer from the available data.

\section{Human papillomavirus}

For the human papillomavirus (HPV), the main question concerning strain replacement is not so much 'how' it could occur but, rather, 'whether' it has occurred or could still occur. In various respects, the results of this thesis have improved our ability to interpret the available data in answering this question. First, concerning pre-vaccination data of co-infection, our results suggest that the commonly observed clustering patterns in both the longitudinal and cross-sectional settings likely imply a limited extent of competition in the mode of direct interaction (Chapter 3). However, we think that there is still ground for the existence of competition through cross-immunity [14, 15], as it could be consistent with the clustering patterns (Chapter 3). Furthermore, such clustering patterns can also be caused by confounding due to common risk factors and so masking competition between HPV types (Chapters 2-4).

Our interpretation concerning the available post-vaccination data is also that it could be still too early to rule out HPV genotype replacement. Although strong cross-protection, e.g. for HPV 31 and 45 by the bivalent vaccine [60], does diminish the likelihood of replacement, findings of smaller extents of positive VE may not preclude the occurrence of replacement (Chapter 6). In addition, while increases in the occurrence of non-vaccine-targeted HPV types have been limited so far [29], it may take a while for substantial strain replacement to occur. This statement is based on the relatively slow transmission dynamics of HPV and the possibility of a honeymoon period preceding strain replacement (Chapter 6).

As genotype replacement in HPV cannot be ruled out yet, the occurrence of the non-targeted HPV types should continually be monitored. Our thesis highlights various aspects around the evaluation and monitoring of HPV genotype replacement that deserve special attention. Evaluation based on the trend of non-vaccine- 
type occurrence is preferable above evaluation based on VE, as evaluation based on the latter is more easily obscured by the present cross-protection, which has been observed for many HPV types (Chapter 6). In particular, HPV types for which negative VE has been observed should be closely monitored, e.g. HPV 39,51 , and 52 [31, 34, 61], as these may be the types with the highest risk for genotype replacement. Countries that have already introduced HPV vaccination should continue their long-standing monitoring studies [31, 62, 63]. In countries yet to initiate HPV vaccination, it is pivotal to measure the baseline occurrence of HPV types before introducing vaccination. Besides data on the occurrence of HPV types, it is also important to collect data on sexual behaviour to disentangle possible secular trends and genotype replacement [37].

Furthermore, some fundamental questions about the existence of competition between HPV genotypes and the way how HPV vaccines work are still unresolved. Firstly, the existence of competition through cross-immunity should be further investigated. Especially longitudinal studies on co-occurrence of HPV types would be helpful for studying cross-immunity (Chapter 3). Cross-sectional studies may be less suitable, as existing serological tests may not be sensitive enough to discriminate between individuals with and without immunity, which is necessary for defining appropriate cross-sectional association measures to infer cross-immunity (Chapter 3). In case evidence for competition remains insufficient, it is important to provide a more in-depth explanation describing how independence between HPV types has been attained from the eco-evolutionary point of view. Lastly, it is useful to investigate further how the extent of vaccine-induced cross-protection for each non-vaccine type depends on the phylogenetic distance to the vaccinetargeted types. Better estimates of the extent of cross-protection could then help us improve prediction of the extent of strain replacement (Chapter 6) [64].

Future findings on these topics will be instrumental in guiding public health decisions. As the currently available vaccines target the most oncogenic HPV types, even if type replacement does occur, the benefit of reducing HPV-related cancers should be much greater than the harm. Nevertheless, knowing the extent of HPV replacement is important for various public health decisions. As the currently available vaccines target different sets of HPV types and confer different levels of cross-protection, the extent of genotype replacement may also differ by vaccines. For instance, the stronger cross-protection provided by the bivalent vaccine than the quadrivalent vaccine (e.g. for HPV 31 and 45) may make the former more robust against strain replacement and increase its comparative cost-effectiveness (with the protection against anogenital warts by the quadrivalent appropriately accounted for) [60]. According to the same logic, if replacement were to occur with the use of the bivalent or the quadrivalent vaccines in oncogenic types that are covered by a nonavalent vaccine, there would be more reason to switch to the higher-valent vaccine despite the higher costs.

Furthermore, the possible occurrence of HPV type replacement also has impli- 
cations for public health decisions about the frequency of cervical screening. Even if replacement occurs to a relatively limited extent, it may still result in numbers of cancer cases at an order of magnitude worthwhile taking into account when determining the optimal frequency of screening. In light of the recent efforts towards the elimination of cervical cancer, it is particularly important to consider any additional burden of cancer that may stem from strain replacement.

As a final note, when conducting research on HPV genotype replacement, researchers should be careful about how to communicate their findings to prevent declining trust in vaccination. After all, the effectiveness of the HPV vaccination on the burden of HPV-related diseases is well supported by data [29, 65]. This is an important point to stress, as strain replacement has often been used as an argument against vaccination, fuelling vaccine hesitancy. HPV vaccination is and has been one of the least well-received additions to the national vaccination programmes with the lowest uptake in many countries. Possible harms due to refusal of vaccination may surpass those due to possible genotype replacement. In addition, a high vaccination coverage may even help to prevent strain replacement from happening by bolstering cross-protection on a population level (Chapter 6).

\section{Other multi-strain pathogens}

The development of strain replacement turned out to be quite different for the two pathogens we considered as case studies. While serotype replacement has been clearly observed for pneumococcus, the situation is more ambiguous for HPV. Experience with vaccination against other multi-strain pathogens is also mixed. For some other multi-strain pathogens, the occurrence of strain replacement is also well observed. For instance, replacement of influenza strains due to antigenic drift occurs on the time scale of seasons [66]. Also for Neisseria meningitidis, replacement in serotype groups and clonal complexes has been observed [67, 68]. For Bordetella pertussis, there has been an emergence of strains with reduced efficacy, especially when using less-reactogenic acellular vaccines [69]. For some other multi-strain pathogens, the occurrence of strain replacement is more ambiguous. For both Haemophilus influenzae and rotavirus, although increases in non-targeted strains have been observed, it is still an ongoing debate whether these increases can be attributed to strain replacement [70-74]. For Haemophilus influenzae, for instance, uncertainty is still present twenty years since the introduction of mass vaccination.

Reviewing the situation of all multi-strain pathogens against which there is vaccination shows that strain replacement is not a rare phenomenon. Neither is it an inevitable outcome, at least based on observations to date. Strain replacement does appear in different forms: increases in the occurrence of already existing strains or the emergence of newly mutated strains; partial or complete replacement; replacement in asymptomatic and/or disease states; quick or slow emer- 
gence; emergence that is clearly observable or ambiguous. Despite the possible offset due to strain replacement, vaccination against multi-strain pathogens has generally been effective in reducing disease burden [75]. This is not a coincidence, as the selection of strains to be included in vaccines is generally based on the strain-specific disease-causing potential.

Nevertheless, for many multi-strain pathogens, there is still room for improvement in the impact of vaccination concerning the burden of the non-targeted strains. For these pathogens, inference of between-strain interaction, and prediction and evaluation of strain replacement are necessary steps towards improvement of the current vaccination strategy. Many of the methodologies considered in this thesis for the inference, prediction, and evaluation could be applicable for other multistrain pathogens, although some findings may need to be adapted for pathogens expressing a more continuous spectrum of strain entities or strong seasonal patterns (e.g. influenza).

Furthermore, although this thesis has mainly focused on strain replacement in pneumococcus and HPV, it does provide some guidance on which aspects to consider when dealing with other multi-strain pathogens. The following questions come to mind for consideration: Is the multi-strain pathogen of interest subject to fast evolution so that emergence of vaccine-resistant strains can be expected? How much niche differentiation has been attained and hence how much do we expect the strains to interfere with one another? How does the pathogen interact with the human body? Does a specific anatomic site of occurrence (i.e. infection or colonisation) impose competition for resources such as space, nutrients, and opportunities to be transmitted? What kind(s) of immune response does the pathogen trigger in the host? Do we expect strain-specific immunity, straintranscending cross-immunity, or both? What is the mode of transmission? Do we expect vaccine-induced cross-protection?

In addition, there are some general strategies that can be adopted to improve the impact of vaccination if strain replacement does occur. Possible strategies include modifying the composition of strains to be targeted in the vaccine to cover the replacing strains with the highest disease burden (as done by the switch to PCV13), switching to a vaccine with a stronger cross-protection for the non-targeted strains, increasing the number of doses to boost the level of crossprotection, or increasing vaccination coverage in the population. For fast mutating pathogens, the vaccine might need to be regularly updated (as is done for the influenza vaccine). For many multi-strain pathogens, the eventual solution may lie in the discovery of a broad-spectrum vaccine that is efficacious against all strains. With such a vaccine, it might even be possible to eradicate the entire pathogen species and so to prevent future emergence of vaccine-resistant strains. Yet, there is always the possibility of replacement at the species level (e.g. between pneumococcus and other respiratory pathogens) [76 77]. This brings our research question back to the wider perspective at the pathogen level. Although we have 
not explicitly considered this, many principles we derived in this thesis are generalisable to interactions and the possibility of vaccine-induced replacement on the species level.

\section{Concluding remarks}

In this thesis, we have investigated whether and how vaccination against a subset of strains of a pathogen would affect the occurrence of the non-targeted strains of the same pathogen, through between-strain interactions. We have considered three methodological problems around this central question: 1) inference of between-strain interactions using pre-vaccination data; 2) prediction of strain replacement using inferred information of between-strain interaction; and 3) evaluation of strain replacement using post-vaccination data. This thesis has contributed to a better understanding of the potential and limitations of the existing methodologies and expanded on them in various directions. In turn, the methodological development has improved our ability to assess the risk of strain replacement for real-world multi-strain pathogens, which may help to guide public health decisions.

This thesis has also brought to light some considerations about public health decision-making around vaccination against multi-strain pathogens. Firstly, our work highlights the importance of considering the total disease burden of all strains of a pathogen when making decisions about vaccination. A well-grounded evaluation of a given vaccination programme should take into account both the possible benefits and harms, hence also the possibility of strain replacement. Although it may be challenging to accurately predict the occurrence of strain replacement before starting to vaccinate, the possibility should not be overlooked. Secondly, public health decision-making regarding vaccination against multi-strain pathogens is likely more a continuous process rather than a one-time action. This is partly due to the unavoidable uncertainty about the possible occurrence of strain replacement before the actual introduction, but it could also be due to the development of new vaccines or other health technologies (e.g. new screening or treatment options). Thirdly, resources should be reserved for conducting research on the assessment of the risk of strain replacement, both for pathogens against which we vaccinate now or might vaccinate in the future. Relevant efforts include the collection and analysis of data for between-strain interactions and data for the impact of vaccination. Ideally, such studies should already start before the introduction of vaccination. Without these studies, it would be impossible to make well-informed decisions about vaccination against multi-strain pathogens.

As a final note, although it is not a goal in itself, vaccination is arguably an ideal ecological experiment from which information about between-strain interactions can be inferred; the occurrence of strain replacement is clear proof of between-strain competition. By observing how vaccination affects the occurrence 
of different pathogen strains, we may come up with new hypotheses and knowledge of how different pathogen strains interact [78]. In turn, improved knowledge of between-strain interaction could generate new ideas of how to improve the current vaccination strategies. All in all, by basing public health decisions on a scientific process, we can gradually learn from our prior decisions and take action to improve the health outcomes of our populations in the future, through, among other things, vaccination.

\section{References}

[1] G. Wunsch, F. Russo, and M. Mouchart. "Do we necessarily need longitudinal data to infer causal relations?" Bulletin of Sociological Methodology 106.1 (2010), 5-18.

[2] S. Telfer, R. Birtles, M. Bennett, et al. "Parasite interactions in natural populations: insights from longitudinal data". Parasitology 135.7 (2008), 767-781.

[3] A. Melegaro, Y. Choi, R. Pebody, et al. "Pneumococcal carriage in United Kingdom families: estimating serotype-specific transmission parameters from longitudinal data". Am J Epidemiol 166.2 (2007), 228-235.

[4] K. Auranen, J. Mehtälä, A. Tanskanen, et al. "Between-strain competition in acquisition and clearance of pneumococcal carriage - epidemiologic evidence from a longitudinal study of day-care children". Am J Epidemiol 171.2 (2010), 169-176.

[5] M. Lipsitch, O. Abdullahi, A. D’Amour, et al. "Estimating rates of carriage acquisition and clearance and competitive ability for pneumococcal serotypes in Kenya with a Markov transition model". Epidemiology 23.4 (2012), 510-519.

[6] J. Mehtälä, M. Antonio, M. S. Kaltoft, et al. "Competition between Streptococcus pneumoniae strains: implications for vaccine-induced replacement in colonization and disease". Epidemiology (2013), 522-529.

[7] K. K. Thomas, J. P. Hughes, J. M. Kuypers, et al. "Concurrent and sequential acquisition of different genital human papillomavirus types". J Infect Dis 182.4 (2000), 1097-1102.

[8] M. C. Rousseau, J. S. Pereira, J. C. Prado, et al. "Cervical coinfection with human papillomavirus (HPV) types as a predictor of acquisition and persistence of HPV infection". $J$ Infect Dis 184.12 (2001), 1508-1517.

[9] P. C. Hill, Y. B. Cheung, A. Akisanya, et al. "Nasopharyngeal carriage of Streptococcus pneumoniae in Gambian infants: a longitudinal study". Clin Infect Dis 46.6 (2008), 807-814.

[10] J. H. Lubin. "Extensions of analytic methods for nested and population-based incident casecontrol studies". J Chronic Dis 39.5 (1986), 379-388.

[11] K. Auranen, H. Rinta-Kokko, and M. E. Halloran. "Estimating strain-specific and overall efficacy of polyvalent vaccines against recurrent pathogens from a cross-sectional study". Biometrics 69.1 (2013), 235-244.

[12] D. P. Durham, E. M. Poolman, Y. Ibuka, et al. "Reevaluation of epidemiological data demonstrates that it is consistent with cross-immunity among human papillomavirus types". J Infect Dis 206.8 (2012), 1291-1298.

[13] T. Malagón, P. Lemieux-Mellouki, J. F. Laprise, et al. "Bias due to correlation between times-atrisk for infection in epidemiologic studies measuring biological interactions between sexually transmitted infections: a case study using human papillomavirus type interactions". Am J Epidemiol 184.12 (2016), 873-883. 
[14] L. F. Xi, Z. R. Edelstein, C. Meyers, et al. "Human papillomavirus types 16 and 18 DNA load in relation to coexistence of other types, particularly those in the same species". Cancer Epidemiol Biomarkers Prev 18.9 (2009), 2507-2512.

[15] J. Biryukov and C. Meyers. "Superinfection exclusion between two high-risk human papillomavirus types during a coinfection". J Virol 92.8 (2018), e01993-17.

[16] N. Mejlhede, B. V. Pedersen, M. Frisch, et al. "Multiple human papilloma virus types in cervical infections: competition or synergy?" APMIS 118.5 (2010), 346-352.

[17] P. S. Wikramaratna, A. Kucharski, S. Gupta, et al. "Five challenges in modelling interacting strain dynamics". Epidemics 10 (2015), 31-34.

[18] A. J. Kucharski, V. Andreasen, and J. R. Gog. "Capturing the dynamics of pathogens with many strains". J Math Biol 72.1 (2016), 1-24.

[19] M. G. M. Gomes, G. F. Medley, and D. J. Nokes. "On the determinants of population structure in antigenically diverse pathogens". Proc Biol Sci 269.1488 (2002), 227-233.

[20] M. Plummer, S. Vaccarella, and S. Franceschi. "Multiple human papillomavirus infections: the exception or the rule? (editorial)". J Infect Dis 203.7 (2011), 891-893.

[21] J. E. Tota, A. V. Ramanakumar, M. Jiang, et al. "Epidemiologic approaches to evaluating the potential for human papillomavirus type replacement postvaccination". Am J Epidemiol 178.4 (2013), 625-634.

[22] T. A. Balan and H. Putter. "Nonproportional hazards and unobserved heterogeneity in clustered survival data: When can we tell the difference?" Stat Med 38.18 (2019), 3405-3420.

[23] A. Melegaro, Y. H. Choi, R. George, et al. "Dynamic models of pneumococcal carriage and the impact of the Heptavalent Pneumococcal Conjugate Vaccine on invasive pneumococcal disease". BMC Infect Dis 10 (2010), 90.

[24] Y. H. Choi, M. Jit, S. Flasche, et al. "Mathematical modelling long-term effects of replacing Prevnar7 with Prevnar13 on invasive pneumococcal diseases in England and Wales". PLOS One 7.7 (2012), e39927.

[25] C. Bottomley, A. Roca, P. C. Hill, et al. "A mathematical model of serotype replacement in pneumococcal carriage following vaccination". J R Soc Interface 10.89 (2013), 20130786.

[26] E. H. Elbasha and A. P. Galvani. "Vaccination against multiple HPV types". Math Biosci 197.1 (2005), 88-117.

[27] M. T. Sofonea, S. Alizon, and Y. Michalakis. "From within-host interactions to epidemiological competition: a general model for multiple infections". Philos Trans $R$ Soc Lond B Biol Sci 370.1675 (2015), 20140303.

[28] M. Lipsitch, C. Colijn, T. Cohen, et al. "No coexistence for free: neutral null models for multistrain pathogens". Epidemics 1.1 (2009), 2-13.

[29] M. Drolet, É. Bénard, N. Pérez, et al. "Population-level impact and herd effects following the introduction of human papillomavirus vaccination programmes: updated systematic review and meta-analysis". Lancet 394.10197 (2019), 497-509.

[30] D. M. Weinberger, R. Malley, and M. Lipsitch. "Serotype replacement in disease after pneumococcal vaccination". Lancet 378.9807 (2011), 1962-1973.

[31] P. J. Woestenberg, A. J. King, B. H. Van Benthem, et al. "Bivalent vaccine effectiveness against type-specific HPV positivity: evidence for cross-protection against oncogenic types among Dutch STI clinic visitors". J Infect Dis 217.2 (2018), 213-222.

[32] A. R. Mclean. "Vaccination, evolution and changes in the efficacy of vaccines: a theoretical framework". Proc Biol Sci 261.1362 (1995), 389-393.

[33] M. Brisson and W. J. Edmunds. "Economic evaluation of vaccination programs: the impact of herd-immunity". Med Decis Making 23.1 (2003), 76-82. 
[34] P. Gray, T. Luostarinen, S. Vänskä, et al. "Occurrence of human papillomavirus (HPV) type replacement by sexual risk-taking behaviour group: Post-hoc analysis of a community randomized clinical trial up to 9 years after vaccination (IV)". Int J Cancer 145.3 (2019), 785796.

[35] J. A. Lewnard and W. P. Hanage. "Making sense of differences in pneumococcal serotype replacement". Lancet Infect Dis 19.6 (2019), e213-e220.

[36] W. P. Hausdorff, B. Hoet, and R. A. Adegbola. "Predicting the impact of new pneumococcal conjugate vaccines: serotype composition is not enough". Expert Rev Vaccines 14.3 (2015), 413-428.

[37] L. Ding, L. E. Widdice, and J. A. Kahn. "Differences between vaccinated and unvaccinated women explain increase in non-vaccine-type human papillomavirus in unvaccinated women after vaccine introduction". Vaccine 35.52 (2017), 7217-7221.

[38] J. E. Tota, F. Struyf, M. Merikukka, et al. "Evaluation of type replacement following HPV16/18 vaccination: pooled analysis of two randomized trials". J Natl Cancer Inst 109.7 (2017), djw300.

[39] M. Saccucci, E. L. Franco, L. Ding, et al. "Non-vaccine-type human papillomavirus prevalence after vaccine introduction: no evidence for type replacement but evidence for cross-protection". Sex Transm Dis 45.4 (2018), 260-265.

[40] N. V. Torres and G. Santos. "The (mathematical) modeling process in biosciences". Front Genet 6 (2015), 354.

[41] V. V. Ganusov. "Strong inference in mathematical modeling: a method for robust science in the twenty-first century". Front Microbiol 7 (2016), 1131.

[42] S. W. Lo, R. A. Gladstone, A. J. Van Tonder, et al. "Pneumococcal lineages associated with serotype replacement and antibiotic resistance in childhood invasive pneumococcal disease in the post-PCV13 era: an international whole-genome sequencing study". Lancet Infect Dis 19.7 (2019), 759-769.

[43] G. Hanquet, P. Krizova, P. Valentiner-Branth, et al. "Effect of childhood pneumococcal conjugate vaccination on invasive disease in older adults of 10 European countries: implications for adult vaccination". Thorax 74.5 (2019), 473-482.

[44] C. Colijn, J. Corander, and N. J. Croucher. "Designing ecologically optimized pneumococcal vaccines using population genomics". Nat Microbiol (2020), 473-485.

[45] M. Nurhonen and K. Auranen. "Optimal serotype compositions for pneumococcal conjugate vaccination under serotype replacement". PLoS Comput Biol 10.2 (2014), e1003477.

[46] F. Hoti, P. Erästö, T. Leino, et al. "Outbreaks of Streptococcus pneumoniae carriage in day care cohorts in Finland - implications for elimination of transmission". BMC Infect Dis 9 (2009), 102.

[47] P. Erästö, F. Hoti, and K. Auranen. "Modeling transmission of multitype infectious agents: application to carriage of Streptococcus pneumoniae". Stat Med 31.14 (2012), 1450-1463.

[48] P. Turner, J. Hinds, C. Turner, et al. "Improved detection of nasopharyngeal cocolonization by multiple pneumococcal serotypes by use of latex agglutination or molecular serotyping by microarray". J Clin Microbiol 49.5 (2011), 1784-1789.

[49] C. Satzke, E. M. Dunne, B. D. Porter, et al. "The PneuCarriage project: a multi-centre comparative study to identify the best serotyping methods for examining pneumococcal carriage in vaccine evaluation studies". PLoS Med 12.11 (2015), e1001903.

[50] C. P. Olwagen, P. V. Adrian, and S. A. Madhi. "Comparison of traditional culture and molecular qPCR for detection of simultaneous carriage of multiple pneumococcal serotypes in African children". Sci Rep 7.1 (2017), 4628.

[51] S. Cobey and M. Lipsitch. "Niche and neutral effects of acquired immunity permit coexistence of pneumococcal serotypes". Science 335.6074 (2012), 1376-1380. 
[52] R. Malley, K. Trzciński, A. Srivastava, et al. "CD4+ T cells mediate antibody-independent acquired immunity to pneumococcal colonization". Proc Natl Acad Sci U S A 102.13 (2005), 4848-4853.

[53] K. Trzciński, Y. Li, D. M. Weinberger, et al. "Effect of serotype on pneumococcal competition in a mouse colonization model". mBio 6.5 (2015), e00902-15.

[54] S. M. Granat, J. Ollgren, E. Herva, et al. "Epidemiological evidence for serotype-independent acquired immunity to pneumococcal carriage". J Infect Dis 200.1 (2009), 99-106.

[55] J. Corander, C. Fraser, M. U. Gutmann, et al. "Frequency-dependent selection in vaccineassociated pneumococcal population dynamics". Nat Ecol Evol 1.12 (2017), 1950-1960.

[56] N. L. Hiller and R. Sá-Leão. "Puzzling over the pneumococcal pangenome". Front Microbiol 9 (2018), 2580.

[57] S. Flasche, O. L. P. de Waroux, K. L. O'Brien, et al. "The serotype distribution among healthy carriers before vaccination is essential for predicting the impact of pneumococcal conjugate vaccine on invasive disease". PLoS Comput Biol 11.4 (2015), e1004173.

[58] D. M. Weinberger, J. L. Warren, T. Dalby, et al. "Differences in the impact of pneumococcal serotype replacement in individuals with and without underlying medical conditions". Clin Infect Dis 69.1 (2019), 100-106.

[59] S. Flasche, W. J. Edmunds, E. Miller, et al. "The impact of specific and non-specific immunity on the ecology of Streptococcus pneumoniae and the implications for vaccination". Proc Biol Sci 280.1771 (2013), 20131939.

[60] D. R. Brown, E. A. Joura, G. P. Yen, et al. "Systematic literature review of cross-protective effect of HPV vaccines based on data from randomized clinical trials and real-world evidence". Vaccine (2021).

[61] F. Carozzi, D. Puliti, C. Ocello, et al. "Monitoring vaccine and non-vaccine HPV type prevalence in the post-vaccination era in women living in the Basilicata region, Italy". BMC Infect Dis 18.1 (2018), 1-11.

[62] K. Kavanagh, K. G. Pollock, K. Cuschieri, et al. "Changes in the prevalence of human papillomavirus following a national bivalent human papillomavirus vaccination programme in Scotland: a 7-year cross-sectional study". Lancet Infect Dis 17.12 (2017), 1293-1302.

[63] C. Patel, J. M. L. Brotherton, A. Pillsbury, et al. "The impact of 10 years of human papillomavirus (HPV) vaccination in Australia: what additional disease burden will a nonavalent vaccine prevent?" Euro Surveill 23.41 (2018), 1700737.

[64] J. A. Bogaards, P. Van Der Weele, P. J. Woestenberg, et al. "Bivalent human papillomavirus (HPV) vaccine effectiveness correlates with phylogenetic distance from HPV vaccine types 16 and 18". J Infect Dis 220.7 (2019), 1141-1146.

[65] J. Lei, A. Ploner, K. M. Elfström, et al. "HPV vaccination and the risk of invasive cervical cancer". N Engl J Med 383.14 (2020), 1340-1348.

[66] F. Carrat and A. Flahault. "Influenza vaccine: the challenge of antigenic drift". Vaccine 25.39-40 (2007), 6852-6862.

[67] R. Booy, A. Gentile, M. Nissen, et al. "Recent changes in the epidemiology of Neisseria meningitidis serogroup $W$ across the world, current vaccination policy choices and possible future strategies". Hum Vaccin Immunother 15.2 (2019), 470-480.

[68] C. O. Buckee, K. A. Jolley, M. Recker, et al. "Role of selection in the emergence of lineages and the evolution of virulence in Neisseria meningitidis". Proc Natl Acad Sci U S A 105.39 (2008), 15082-15087.

[69] G. A. M. Berbers, S. C. de Greeff, and F. R. Mooi. "Improving pertussis vaccination". Hum Vaccin 5.7 (2009), 497-503. 
[70] M. Lipsitch. "Bacterial vaccines and serotype replacement: lessons from Haemophilus influenzae and prospects for Streptococcus pneumoniae". Emerg Infect Dis 5.3 (1999), 336-345.

[71] H. Adam, S. Richardson, F. Jamieson, et al. "Changing epidemiology of invasive Haemophilus influenzae in Ontario, Canada: evidence for herd effects and strain replacement due to Hib vaccination". Vaccine 28.24 (2010), 4073-4078.

[72] M. Ulanova. "Invasive Haemophilus influenzae serotype a disease in the $H$. influenzae Serotype b Conjugate Vaccine Era: Where Are We Going? (commentary)". Clin Infect Dis (2020).

[73] G. L. Bibera, J. Chen, P. Pereira, et al. "Dynamics of G2P [4] strain evolution and rotavirus vaccination: A review of evidence for Rotarix". Vaccine 38.35 (2020), 5591-5600.

[74] M. Iturriza-Gómara, T. Dallman, K. Bányai, et al. "Rotavirus genotypes co-circulating in Europe between 2006 and 2009 as determined by EuroRotaNet, a pan-European collaborative strain surveillance network". Epidemiol Infect 139.6 (2011), 895-909.

[75] M. Drolet, É. Bénard, M. Jit, et al. "Model comparisons of the effectiveness and cost-effectiveness of vaccination: a systematic review of the literature". Value Health 21.10 (2018), 1250-1258.

[76] J. O. Lloyd-Smith. "Vacated niches, competitive release and the community ecology of pathogen eradication". Philos Trans R Soc B Biol Sci 368.1623 (2013), 20120150.

[77] D. Bogaert, A. van Belkum, M. Sluijter, et al. "Colonisation by Streptococcus pneumoniae and Staphylococcus aureus in healthy children". Lancet 363.9424 (2004), 1871-1872.

[78] W. P. Hausdorff and W. P. Hanage. "Interim results of an ecological experiment - Conjugate vaccination against the pneumococcus and serotype replacement". Hum Vaccin Immunother 12.2 (2016), 358-374. 
Supplements 


\section{Nederlandse samenvatting}

Pathogenen bestaan vaak uit meerdere stammen. Van sommige pathogenen leven de verschillende stammen niet gescheiden, maar beïnvloeden elkaars voorkomen. Wanneer stammen van een pathogeen met elkaar in interactie zijn, zal een vaccin dat bescherming biedt tegen een deel van deze stammen ook invloed hebben op het voorkomen van stammen die niet in het vaccin zijn opgenomen. Zo kan synergie tussen stammen leiden tot een afname in het voorkomen van de nonvaccinstammen. Daarentegen kan competitie tussen stammen leiden tot een toename in het voorkomen van non-vaccinstammen, wat ook wel 'stamvervanging' genoemd wordt.

In dit proefschrift beschouwen we de methodologie voor de volgende drie problemen:

1. afleiding van interacties tussen stammen uit pre-vaccinatie data,

2. voorspelling van stamvervanging op basis van informatie over interacties tussen stammen,

3. evaluatie van stamvervanging uit post-vaccinatie data.

Hierbij hebben we Streptococcus pneumoniae (pneumokokken) en het humaan papillomavirus (HPV) genomen als leidende casestudies.

\section{Afleiding van interacties tussen stammen}

In dit proefschrift hebben we methodes voor de afleiding van interacties tussen stammen uit longitudinale en cross-sectionele data beschouwd. Uit longitudinale data zijn deze interacties te schatten met behulp van multi-state modellen, welke echter rekenintensief zijn. Hierdoor was analyse vooralsnog beperkt tot data met dragerschap van maximaal twee stammen tegelijkertijd. In Hoofdstuk 2 hebben we bestaande methodes aangepast door de waarschijnlijkheidsfunctie te benaderen. Hiermee is het mogelijk om data met observaties van meer dan twee stammen tegelijkertijd te analyseren.

In de longitudinale setting worden interacties tussen stammen doorgaans geschat als ratio's die hazards van acquisitie of klaring van verschillende stammen in de aan- en afwezigheid van andere stammen vergelijken. In de cross-sectionele setting is het afzonderlijk schatten van interacties in acquisitie en klaring niet mogelijk. Wel kan de gezamenlijke kracht van de beide interactie modi geschat worden als een odds-ratio, de ratio die de odds van het voorkomen van bepaalde stammen in de aan- en afwezigheid van andere stammen vergelijkt. Met behulp van algebraïsche argumenten in een transmissiemodel met twee stammen hebben we in Hoofdstuk 3 afgeleid dat deze longitudinale en cross-sectionele 
maten voor tussen-stam interacties, de hazard-ratio's en odds-ratio's, met elkaar overeenkomen in een endemische situatie, oftewel de stabiele toestand van het systeem. In Hoofdstuk 4 hebben we aangetoond dat deze overeenkomstigheid ook geldt wanneer er meer dan twee stammen zijn, in welk geval interacties tussen stammen geschat kunnen worden met behulp van netwerkmodellen. Hiermee hebben we aangetoond dat cross-sectionele data ook bruikbaar kunnen zijn voor het afleiden van tussen-stam interacties.

Er zijn ook situaties waarin de genoemde overeenkomstigheid tussen de longitudinale en cross-sectionele maten voor interacties tussen stammen niet geldt. In Hoofdstuk 4 hebben we laten zien dat dit het geval is als stammen met elkaar in competitie zijn door middel van kruisimmuniteit die geïnduceerd wordt na klaring van infectie of dragerschap. Wanneer de odds-ratio gebruikt wordt als maat, leidt deze modus van competitie juist tot een positieve associatie in het voorkomen van verschillende stammen, en is daarmee niet meer te onderscheiden van synergetische interactie. Bij pathogenen waarbij kruisimmuniteit een reële mogelijkheid is, is het daarom raadzaam om interacties tussen stammen af te leiden uit longitudinale data in plaats van cross-sectionele data.

Verder kan 'confounding' (observationele verstoring) door gemeenschappelijke risicofactoren leiden tot systematische fouten richting positieve associaties in het voorkomen van verschillende stammen. Hierdoor kunnen competitieve interacties gemaskeerd worden in positieve associaties. Confounding kan verholpen worden door te corrigeren voor gemeenschappelijke risicofactoren in de statistische analyses. In Hoofdstuk 4 hebben we laten zien hoe waargenomen gemeenschappelijke risicofactoren meegenomen kunnen worden in netwerkmodellen als variabelen die de kans op het voorkomen van alle stammen modificeren. Voor ongemeten risicofactoren kan ook gecorrigeerd worden met behulp van persoonsgebonden 'random effecten' die de hazards (van acquisitie of klaring) van alle stammen modificeren, zoals gedemonstreerd in Hoofdstuk 2.

\section{Voorspelling van stamvervanging}

Voorspelling van stamvervanging is mogelijk met informatie over interacties tussen stammen, afgeleid uit longitudinale of cross-sectionele data. In Hoofdstuk 5 hebben we hiervoor een nieuw voorspellingsraamwerk ontwikkeld.

Het voorgestelde raamwerk biedt voorspellers voor stamvervanging van zowel ieder non-vaccinstam afzonderlijk als voor groepen van non-vaccinstammen. De voorspeller voor specifieke stammen voegt de mogelijk heterogene interacties tussen de gegeven non-vaccinstam en de verschillende vaccinstammen samen. Ook houdt de voorspeller rekening met de interacties tussen de verschillende nonvaccinstammen onderling. De voorspeller voor een groep van stammen voegt samen de voorspellers voor alle stammen samen die binnen de gegeven groep vallen. De voorgestelde voorspellers zijn geconstrueerd op basis van de ratio 
tussen de hazard-ratio's voor interacties tussen stammen in acquisitie en klaring, of op basis van en de corresponderende odds-ratio's. In Hoofdstuk 2 hebben we de voorspellende kracht van deze maat voor stamvervanging in een generiek model voor twee stammen laten zien. Deze wordt tevens bevestigd in een gedetailleerde simulatiestudie in Hoofdstuk 5.

\section{Evaluatie van stamvervanging}

Hoofdstuk 6 behandelt de evaluatie van stamvervanging met surveillance data na de introductie van vaccinatie. In een simulatiestudie hebben we de validiteit van twee veel gebruikte maten van stamvervanging onderzocht. De eerste maat is de ratio tussen de prevalentie van de non-vaccinstammen in de pre- en postvaccinatie periodes. De tweede maat is de ratio tussen het risico op non-vaccinstammen in gevaccineerde en ongevaccineerde individuen. De ongevaccineerde individuen zijn hierbij ook afkomstig uit de post-vaccinatie periode.

We tonen aan dat beide maten bruikbaar zijn voor de evaluatie van stamvervanging als het vaccin geen kruisbescherming biedt voor de non-vaccinstammen. In aanwezigheid van vaccin-geïnduceerde kruisbescherming is de validiteit echter niet gewaarborgd. In deze situatie kan de prevalentie van non-vaccinstammen meteen na de introductie van vaccinatie eerst dalen alvorens om te slaan in stamvervanging. In de literatuur wordt de initiële daling ook wel een 'honeymoon period' van vaccinatie genoemd. Voor een accurate evaluatie van stamvervanging is het daarom van belang dat de opvolgtijd lang genoeg is. De gepaste opvolgtijd kan onder andere afhankelijk zijn van de vaccinatiegraad, de manier van overdracht en de modus van interactie. Ook kan evaluatie van stamvervanging op basis van de ratio tussen risico op non-vaccinstammen in gevaccineerde en ongevaccineerde individuen verstoord worden door de aanwezigheid van vaccingeïnduceerde kruisbescherming. Gevaccineerde individuen kunnen namelijk een blijvend lager risico op non-vaccinstammen houden dan onder de ongevaccineerde individuen, terwijl het risico in de gehele populatie gestegen is door stamvervanging.

\section{Discussie van de methodologische bevindingen}

Hoofdstuk 7, de algemene discussie, begint met het samenvatten en bediscussiëren van de methodologische bevindingen. In dit proefschrift tonen we aan dat interacties tussen stammen kunnen worden afgeleid uit zowel longitudinale als crosssectionele data. Deze interacties zijn te kwantificeren als hazard-ratio's en oddsratio's, respectievelijk. De correspondentie van deze maten is afhankelijk van de zogenoemde reversibiliteit van het onderliggende dynamische systeem voor wat betreft acquisitie en klaring van pathogeen stammen. Zowel longitudinale als cross-sectionele data bieden voor- en nadelen voor afleiding van interacties 
tussen stammen. Longitudinale data zijn meest geschikt voor identificatie van verschillende modi van interactie, alsmede correctie voor gezamenlijke risicofactoren voor verschillende stammen. Cross-sectionele data zijn veelal gemakkelijker te verkrijgen en omvatten vaak een groot aantal studiedeelnemers, wat de statistische power van analyses ten goede komt.

De in dit proefschrift beschreven statistische modellen bieden de mogelijkheid om paarsgewijze interacties tussen stammen te schatten, conditioneel op interacties met andere stammen. Dit is in contrast met marginale methodes voor afleiding van tussen-stam interacties, waarin stammen telkens paarsgewijs worden beoordeeld zonder correctie voor aanwezigheid van overige stammen. Deze methodes zijn vaak minder rekenintensief, maar vatbaarder voor systematische fouten. Idealiter moeten resultaten van marginale methodes bevestigd worden met nietmarginale methodes, zoals multi-state modellen en netwerkmodellen. Deze modellen bieden ook de mogelijkheid om heterogeniteit van interacties tussen stammen te onderzoeken, alsmede manieren waarop interacties over meerdere stammen optellen. Deze aspecten zijn in dit proefschrift aangestipt en verdienen verder onderzoek, mede vanwege de relevantie voor stamvervanging.

Het door ons voorgestelde voorspellingsraamwerk voor stamvervanging biedt voordelen in vergelijking met alternatieven, die gebaseerd zijn op expliciete dynamische modellering van meerdere stammen tegelijkertijd. Het benodigt geen extra behalve dat schattingen van interactie tussen stammen en is minder rekenintensief. In tegenstelling is dynamische modellering beter geschikt om gedetailleerde voorspelling te bieden over stamvervanging. Dynamische aspecten van multi-stam transmissie hebben ook invloed op de timing waarin stamvervanging optreedt en daarmee ook op opvolgtijd wat nodig is om stamvervanging te evalueren in de praktijk.

\section{Implicaties voor specifieke pathogenen}

In Hoofdstuk 7 worden ook de implicaties van de methodologische bevindingen voor de multi-stam pathogenen in de echte wereld besproken, met bijzondere aandacht voor de twee casestudies: pneumokokken en HPV.

Door de in Hoofdstuk 2 ontwikkeld schattingsmethode toe te passen op een dataset van pneumokokkendragerschap hebben we nieuwe inzichten verkregen over de wijze waarop verschillende stammen met elkaar concurreren. Mogelijk is competitie in klaring een belangrijkere modus van interactie dan competitie in acquisitie. Verder suggereren onze bevindingen een beperkte mate van concurrentie tijdens dragerschap. Mogelijk speelt competitie middels andere modi (bijv. door kruisimmuniteit tussen stammen) een belangrijkere rol dan tot dusver verondersteld. Meer onderzoek is nodig om de precieze wijze waarop verschillende stammen van pneumokokken met elkaar concurreren te beschrijven. Deze kennis is noodzakelijk voor het verbeteren van de bestaande modellen voor stamvervang- 
ing. Betere modellen kunnen ons helpen om nieuwe vaccins en vaccinatiestrategieën te ontwikkelen die de ziektelast van pneumokokken verder omlaag kunnen brengen.

Voor HPV wijzen onze resultaten erop dat stamvervanging niet geheel is uit te sluiten op basis van de huidige evidentie. Zo zijn de (over het algemeen) positieve associaties in het voorkomen van meerdere stammen consistent met een scenario van natuurlijke kruisimmuniteit. Evaluatie van stamvervanging op basis van post-vaccinatie data wordt daarnaast bemoeilijkt door het bestaan van vaccin-geïnduceerde kruisbescherming. Een langere opvolgtijd is nodig om uitsluitsel te geven. Surveillancestudies die het voorkomen van non-vaccinstammen monitoren blijven essentieel. Ook dient de mate van vaccin-geïnduceerde kruisbescherming en de mate waarop verschillende HPV-stammen met elkaar concurreren middels kruisimmuniteit verder onderzocht te worden. Om de geschikte frequentie van screening op baarmoederhalskanker in het post-vaccinatietijdperk te kunnen vaststellen is het essentieel om de resterende ziektelast van de nonvaccinstammen te kunnen kwantificeren, inclusief het risico op stamvervanging.

Door het bestuderen van stamvervanging bij pneumokokken en HPV hebben we de belangrijke aspecten en vraagstukken geïdentificeerd die ook nuttig zijn voor de beoordeling van het risico van stamvervanging bij andere pathogenen. Daarnaast zijn we algemene strategieën tegengekomen die de kans of mate van stamvervanging bij andere pathogenen kunnen beperken. Deze hebben betrekking op verhoging van de vaccinatiegraad, verbetering van vaccineffectiviteit, of bredere werkzaamheid van vaccins, bijv. door verhoging van de valentie of versterking van kruisbescherming.

In het kader van de besluitvorming over vaccinatie tegen multi-stam pathogenen is een herhaald proces van evaluatie onoverkomelijk. Gemaakte aannames zullen geëvalueerd moeten worden wanneer de effecten van vaccinatie meetbaar worden of wanneer technologische vooruitgang geboekt wordt, zoals de ontdekking van nieuwe vaccins met brede werkzaamheid. Een gunstige bijkomstigheid van nieuwe, grootschalige vaccinatiestrategieën is dat deze weer leiden tot nieuwe observaties over interacties tussen stammen, en zo ook tot nieuwe inzichten over de ecologie van multi-stam pathogenen. Door nieuwe inzichten uit gemaakte beslissingen in acht te nemen, hebben we het vermogen om nieuwe beslissingen te maken die de volksgezondheid steeds verder verbeteren. 


\section{Acknowledgements}

During the years of my PhD research, I have come to realise the importance of many people in my work and other aspects of life. Following are some words of gratitude towards these people.

Beste Hans, voordat ik je ga bedanken voor je rol als co-promotor, moet ik je eerst bedanken voor het schrijven van een ontzettend uitdagende onderzoekvoorstel waar ik afgelopen zes jaar mijn hoofd over mag breken. Je hebt niet alleen gezorgd voor de totstandkoming van mijn promotieonderzoek, maar ook voor een goede afloop; je bijdrage was beslissend geweest van het begin tot einde. Ik heb veel genoten van de vrijheid die je mij hebt gegeven om mijn interesse te volgen. Behalve je inhoudelijk sterke bijdrage ben ik je ook erg dankbaar voor je merkbare aanmoedigingen, waardering en erkenning. Al deze elementen waren essentieel geweest in mijn groei tot een onderzoeker. Beste Mirjam, jou wil ik bedanken voor je rol als promotor en voor het overzien van mijn project ervóór. Dank je voor de bijdrage aan mijn proefschrift. Ik ben erg blij met jou als promotor! Beste Jacco, ook jou wil ik bedanken voor je begeleiding. Bedankt voor je tijd en bijdrage aan mijn promotieonderzoek.

Dear Kari and Simopekka, thank you for the generosity of your time and involvement in my PhD project. Doing research with you both has been most instructive and enjoyable. Other than that, I am also very grateful for your warm hospitality during my visits to Finland. Dear Matti, I would also like to thank you for your interest and involvement in my research. Kiitos paljon! Dear Krzysztof, Elisa and Kishan, thank you for your contribution to the thesis. Beste leescommissie, bedankt voor het lezen en beoordelen van dit proefschrift.

Collega's bij RIVM, velen van jullie wil ik bedanken. Werken bij het RIVM was een ontzettend leerzame ervaring. Het heeft me veel geleerd over epidemiologie en werken in een onderzoeksinstituut. Collega's bij MOD, jullie wil ik in het bijzonder bedanken. Wat was het leuk om met jullie in een team te zitten. Jullie kritische houding en aanstekelijke passie voor onderzoek hebben mij zeker een betere onderzoek gemaakt. Bedankt ook voor alle hulp bij het oplossen van sommetjes, problemen met R-scriptjes en de feedback op posters en presentaties. Ook wil ik alle collega's bij EPI bedanken voor de fijne sfeer op werk, voor de gezellige lunches op de zonnige terras of bij de markt, voor de koffiepauzes, en voor de gesprekken in het gangpad. Speciale dank gaat uit naar mijn liefste kamergenootjes! Jullie zijn er altijd om even ergens over te brainstormen, om naar elkaars verhaal te luisteren, en om iets gezelligs mee te doen! Other colleagues and friends I have met through UU, LUMC, THL and IARC, my thanks extend to all of you! Dear lacopo, thank you for the opportunity to finalize my thesis at IARC while further developing myself as a researcher.

Beste paranimf, lieve Petra, wat was het fijn om samen een gedeelte van onze 
$\mathrm{PhD}$ tijd samen door te brengen! Je nuchtere houding en gezelligheid deden mij altijd goed, daarbij natuurlijk ook alle koekjes, theetjes en bitterballen samen! Beste paranimf, lieve Bo, ontzettend bedankt voor de vriendschap, voor het delen van zowel leuke als zware momenten. Dank je voor je wijze en eerlijke adviezen. Ze zijn soms niet wat ik wil horen, maar wel altijd wat ik nodig heb!

Beste Carmen, Evy, Marieke, Jiayi, Anja, Janet, Yuklam, Danny, Annie, Chee, Jimmy, Joey, Patrick, Syreeta, Omar, Rick, Lloyd, Lotte, Marie, Justin, Gwylim, Elske, Ann-Marie en Ramon, dank jullie allemaal de steun gedurende deze $\mathrm{PhD}$ jaren; thank you all for the support during these $\mathrm{PhD}$ years. Daarbij, Evy, bedankt voor de super gave cover; Gywlim en Lloyd, voor de muzikale afleiding; Carmen, voor de facetime; Marie; voor de lieve kaartjes; Yuklam en Danny, voor de interesse in mijn onderzoek en de studietijd samen; Justin, for proofreading; Elske, Ann-Marie and Ramon, for sharing a home where I can feel comfortable and recharge for work; celgroep, bedankt voor alle aanmoedigingen en gebed!

Lieve broer, dank je dat ik altijd op je kan rekenen. $\mathrm{Pa}$, ma, ik ben jullie dankbaar voor de vrijheid die jullie mij hebben gegeven om mijn opleiding helemaal zelf in te vullen en mij daarin te steunen. Bedankt voor jullie onvoorwaardelijke steun en liefde. 


\section{Curriculum vitae}

Irene Man was born on December 8th 1990 in Hong Kong and moved to the Netherlands at the age of eleven. She attended the high school Augustinianum in Eindhoven. There, her interest in mathematics sparked, which led her to study mathematics at the Eindhoven University of Technology in 2009. In 2015, she completed her university study with a master in Statistics, Probability and Operations Research and a master thesis on predictive maintenance of imaging systems at Philips Healthcare. After graduation, wishing to work more

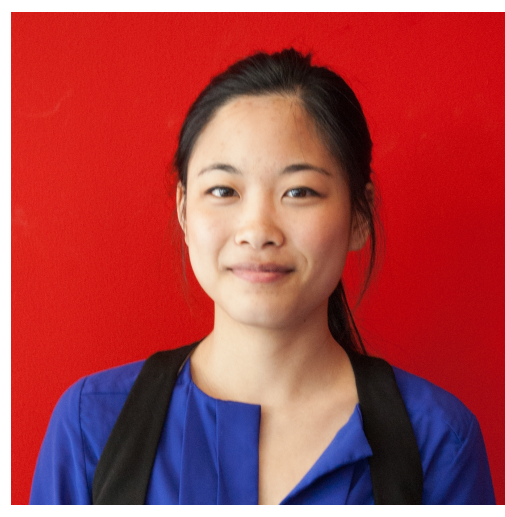
on health-related topics, she started her $\mathrm{PhD}$ research on infectious disease modelling at the National Institute for Public Health and the Environment (RIVM). Her $\mathrm{PhD}$ research focused on modelling multi-strain pathogen dynamics and vaccineinduced strain replacement. As a part of her PhD research, she visited the Finnish Institute for Health and Welfare. During her PhD programme, she had the opportunity to attend many summer schools, workshops and conferences. In 2016 and 2019 , she participated in the organisation of a series of two workshops on multistrain pathogen dynamics at the RIVM. Currently, she continues to work in the field of infectious disease modelling as a post-doctoral fellow at the International Agency for Research on Cancer. 


\section{List of publications}

I. Man, J. Wallinga, and J. A. Bogaards. "Inferring pathogen type interactions using cross-sectional prevalence data: opportunities and pitfalls for predicting type replacement". Epidemiology 29.5 (2018), 666-674.

I. Man, K. Auranen, J. Wallinga, and J. A. Bogaards. "Capturing multiple-type interactions into practical predic- tors of type replacement following human papillomavirus vaccination". Philos Trans R Soc Lond B Biol Sci 374.1773 (2019), 20180298.

P. Gray, T. Luostarinen, S. Vänskä, T. Eriksson, C. Lagheden, I. Man, ..., and M. Lehtinen. "Occurrence of human papillomavirus (HPV) type replacement by sexual risk-taking behaviour group: Post-hoc analysis of a community randomized clinical trial up to 9 years after vaccination (IV)". Int J Cancer, 145.3 (2019), 785-796.

I. Man, S. Vänskä, M. Lehtinen, and J. A. Bogaards. "Human papillomavirus genotype replacement: still too early to tell?". J Infect Dis, (2020), jiaa032.

I. Man, J. A. Bogaards, K. Makwana, K. Trzciński, and K. Auranen. "Approximate likelihood-based estimation method of multiple-type pathogen interactions: an application to longitudinal pneumococcal carriage data". Submitted.

I. Man, E. Benincà, M. E. E. Kretzschmar, and J. A. Bogaards. "Reconstructing heterogeneous pathogen interactions from co-occurrence data via statistical network inference". Submitted. 